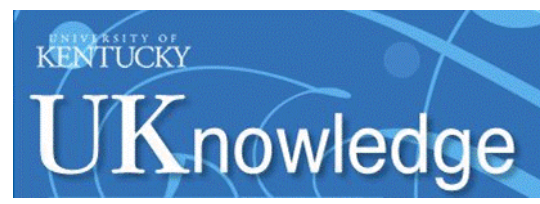

University of Kentucky

UKnowledge

2016

\title{
AN EXPLORATION OF TRANSITION EXPERIENCES SHAPING STUDENT VETERAN LIFE FLOW
}

\author{
Brian T. Gregg \\ University of Kentucky, btgwssu@aol.com \\ Digital Object Identifier: http://dx.doi.org/10.13023/ETD.2016.143
}

Right click to open a feedback form in a new tab to let us know how this document benefits you.

\author{
Recommended Citation \\ Gregg, Brian T., "AN EXPLORATION OF TRANSITION EXPERIENCES SHAPING STUDENT VETERAN LIFE \\ FLOW" (2016). Theses and Dissertations--Rehabilitation Sciences. 31. \\ https://uknowledge.uky.edu/rehabsci_etds/31
}

This Doctoral Dissertation is brought to you for free and open access by the College of Health Sciences at UKnowledge. It has been accepted for inclusion in Theses and Dissertations--Rehabilitation Sciences by an authorized administrator of UKnowledge. For more information, please contact UKnowledge@lsv.uky.edu. 


\section{STUDENT AGREEMENT:}

I represent that my thesis or dissertation and abstract are my original work. Proper attribution has been given to all outside sources. I understand that I am solely responsible for obtaining any needed copyright permissions. I have obtained needed written permission statement(s) from the owner(s) of each third-party copyrighted matter to be included in my work, allowing electronic distribution (if such use is not permitted by the fair use doctrine) which will be submitted to UKnowledge as Additional File.

I hereby grant to The University of Kentucky and its agents the irrevocable, non-exclusive, and royalty-free license to archive and make accessible my work in whole or in part in all forms of media, now or hereafter known. I agree that the document mentioned above may be made available immediately for worldwide access unless an embargo applies.

I retain all other ownership rights to the copyright of my work. I also retain the right to use in future works (such as articles or books) all or part of my work. I understand that I am free to register the copyright to my work.

\section{REVIEW, APPROVAL AND ACCEPTANCE}

The document mentioned above has been reviewed and accepted by the student's advisor, on behalf of the advisory committee, and by the Director of Graduate Studies (DGS), on behalf of the program; we verify that this is the final, approved version of the student's thesis including all changes required by the advisory committee. The undersigned agree to abide by the statements above.

Brian T. Gregg, Student

Dr. Anne Shordike, Major Professor

Dr. Richard Andreatta, Director of Graduate Studies 


\title{
AN EXPLORATION OF TRANSITION EXPERIENCES SHAPING STUDENT VETERAN LIFE FLOW
}

\section{DISSERTATION}

A dissertation submitted in partial fulfillment of the requirements for the degree of Doctor of Philosophy in the College of Health Sciences at the University of Kentucky

By

\author{
Brian Tuan Gregg \\ Lexington, Kentucky
}

Co-Directors: Dr. Anne Shordike, Professor of Occupational Therapy and Dr. Patrick Kitzman, Associate Professor of Physical Therapy

Lexington, Kentucky

2016

Copyright (C Brian Tuan Gregg 2016 


\section{ABSTRACT OF DISSERTATION}

\section{AN EXPLORATION OF TRANSITION EXPERIENCES SHAPING STUDENT VETERAN LIFE FLOW}

Educational institutions offer transformative opportunities for veterans transitioning from military service. Veteran-specific cultural supports in educational environments offer participation in occupations and development of skills needed to complete educational goals. However, veterans experience complex life circumstances atypical from traditional student culture which influences use of available environmental supports. Currently, the student veteran population is growing following fifteen years of combat and with systematic downsizing of the military. Therefore, rehabilitation professionals and educators must understand the unique experiences which shape veteran life flow during the transition process.

This dissertation contains three studies investigating veteran experiences transitioning to postsecondary education. The first study surveyed the attitudes and beliefs of veterans coping with the transition to postsecondary education. Results identified veterans' preference to using skills learned in the military for solving problems in the transition and reported limited use of university supports made available. A second study examined lived experiences of veterans transitioning and their interactions with traditional students and university supports. Results identified veterans emerge in college culture following a transactive process of sorting through military experiences, building on military skills, and developing new relationships for civilian identity.

The final constructivist grounded theory study investigated the individual and collective constructions of veteran transitioning experiences in relation to culture and context. Twelve veterans were theoretically sampled and developed Kawa (River) metaphorical diagrams of their transition experiences. Intensive interviews discussed the meaning ascribed to veterans' diagrams. Interview data was analyzed with the constant comparison technique until theoretical saturation was achieved. 
The emergent theory explained student veteran life flow by two major theoretical concepts: seeking understanding and gaining stability to shape life flow in transition from military to student culture. Veterans shared understanding in each cultural environment by socially interacting with others perceived to be reaching out and connecting with veterans. Veterans also performed autonomous actions of gaining stability by using their skills, pinpointing useful resources, and cultivating the growth and expression of personal skills. Both theoretical processes simultaneously, enhanced veteran's transition to postsecondary education and promoted harmony within veteran life flow over time.

KEYWORDS: Kawa Model, Student Veteran, Transition, Constructivist Grounded Theory, Education

Brian Tuan Gregg

Student's Signature

April 28, 2016

Date 


\section{AN EXPLORATION OF TRANSITION EXPERIENCES SHAPING STUDENT VETERAN LIFE FLOW}

\section{By}

Brian Tuan Gregg

Anne Shordike, Ph.D.

Co-Director of Dissertation

Patrick H. Kitzman, Ph.D.

Co-Director of Dissertation

Richard D. Andreatta, Ph.D Director of Graduate Studies

April 28, 2016

Date 


\section{ACKNOWLEDGEMENTS}

I would like to thank the respective military leaders who endorsed my application and selection for the U.S. Army Long-Term Health Education and Training scholarship. I must express my sincere gratitude towards my advisor and committee chair, Dr. Anne Shordike for all of her mentorship and support in completing this dissertation. Dr.

Shordike has been instrumental in guiding my journey from fledgling research ideas into compelling research questions that culminated into this dissertation. Dr. Shordike has also been instrumental in supporting my transition to school and coordinating efforts to ensure my family's well-being in the process; my family is truly grateful for all of her efforts.

I must also thank Dr. Patrick Kitzman, my committee co-chair, for his teachings and guidance throughout my studies. I have grown by leaps and bounds under his tutelage in developing a consistent line of research questions. Furthermore, he has taught me the importance of clarity, conciseness, and answering that key question of "so what" in each research project. I am truly indebted to him for all that he has imparted onto me. I must also thank Dr. Dana Howell and Dr. Michael Iwama for their support and guidance in my studies.

I thank Dr. Howell for her passion and commitment to excellence. Dr. Howell has consistently supported and challenged me in ways that have only sharpened my perspective. The knowledge and skills that I have developed under her watchful eye are numerous. I am grateful that she has been able to unlock the discrete understandings that I began my journey with and cultivated a shared passion for qualitative research. I must thank Dr. Iwama for all of his lessons of the many uses of metaphors in everyday life. 
From the river to the fruit, my understanding of culture and shared human experience has flourished under his mentorship. Our life stories are forever contextually bound from the work completed in this dissertation. I commend all of my gracious committee members for their investment into my academic endeavors and I aim to ensure they each receive a hearty return on investment.

Lastly, I thank all of my family members who have provided the inspiration and support needed to actualize the completion of my doctoral studies. My family has consistently provided encouragement in all of the goals I have set forth and this work is a testament to their devoted support. Most importantly, I want to thank my loving wife Danyelle. Your compassion and love shared during these challenging times have been appreciated in ways that words cannot even describe. I am beyond grateful for the numerous aspects of giving that you have displayed in cultivating the foundation of our house. Thank you for the constant support and encouragement. I want to also thank our wonderful daughters Inessa and Adelle. Thank you for understanding that daddy's late nights and early mornings were all part of a greater purpose in helping military people just like us. 
TABLE OF CONTENTS

AN EXPLORATION OF TRANSITION EXPERIENCES

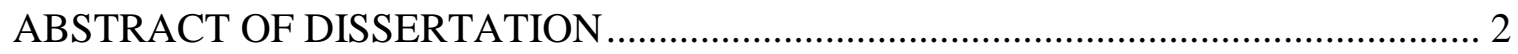

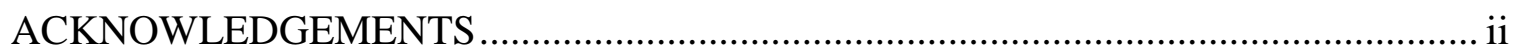

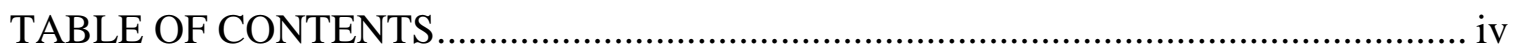

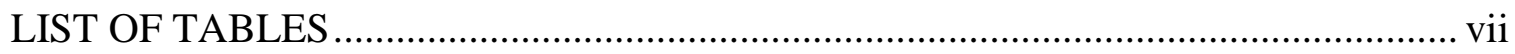

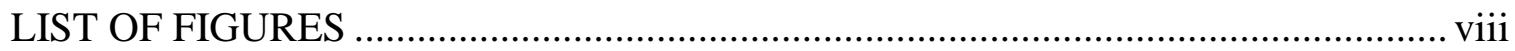

Chapter One: An Introduction to Veteran Transitions...................................................... 1

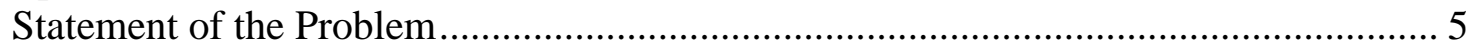

Statement of Purpose and Research Questions ………............................................ 5

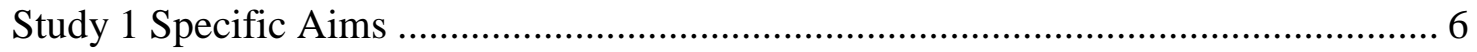

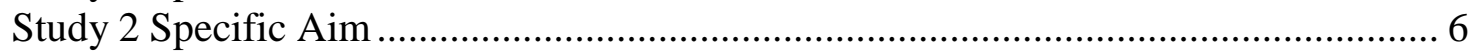

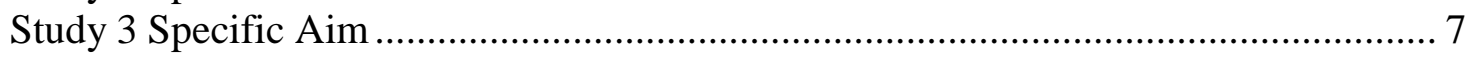

Research Approach ................................................................................................ 7

Theoretical Framework: The Kawa (Japanese for River) Model .................................. 9

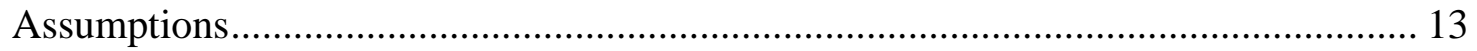

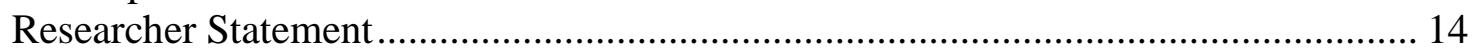

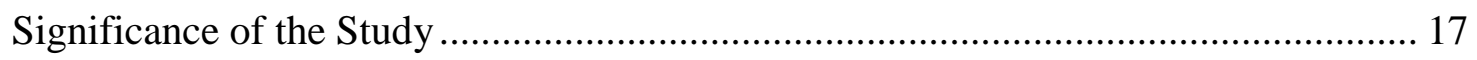

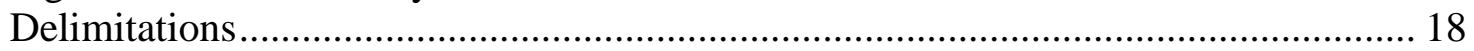

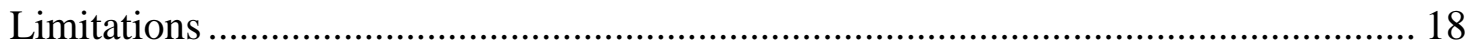

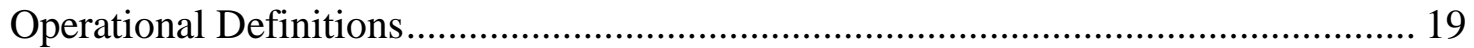

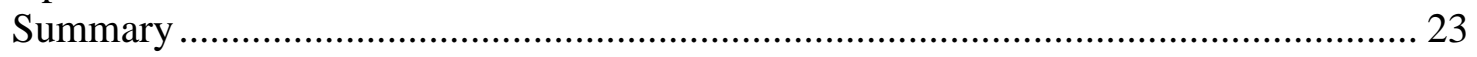

Chapter Two: Review of the Literature ……………................................................ 24

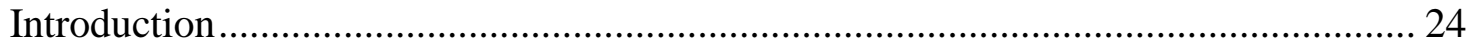

Measurement of Veteran Transition Challenges........................................................... 24

Veteran Transitions into Gainful Employment .............................................................. 27

Veteran Transitions into Postsecondary Education ...................................................... 30

Transition Opportunities for Occupational Therapy …………………………............ 34

Implications for Occupational Therapy Practice ………………………................ 36

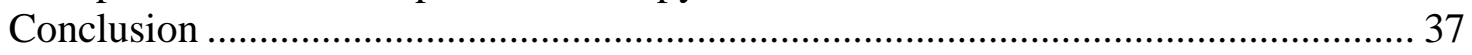

Chapter Three: Well-being and Coping of Student Veterans Readjusting Into Academia:

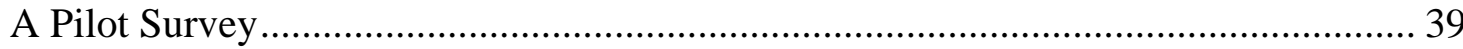

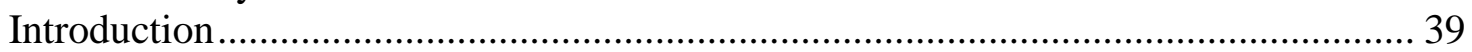

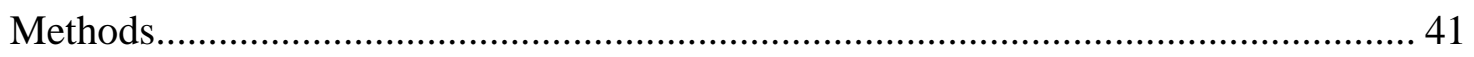

Adult Transition Framework............................................................................. 41

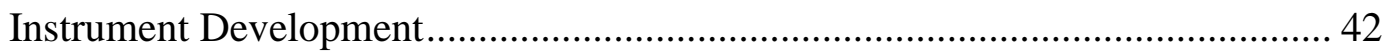




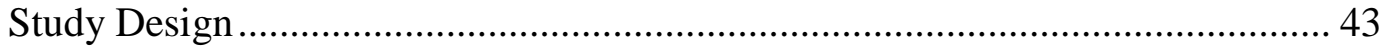

Subject Recruitment .............................................................................. 43

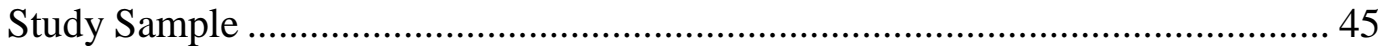

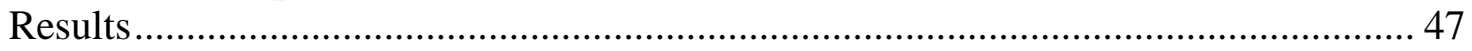

Factor Items Representing Situation .............................................................. 49

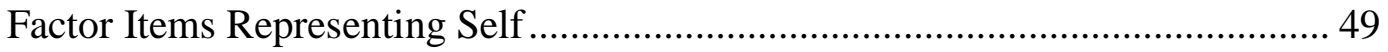

Factor Items Representing Support............................................................... 50

Factor Items Representing Strategies............................................................ 52

Open-Ended Responses ................................................................................... 52

Relational Challenges. .............................................................................. 53

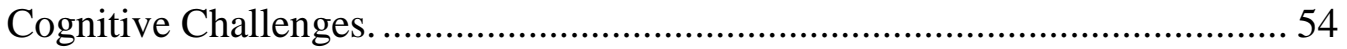

Financial Challenges................................................................................. 54

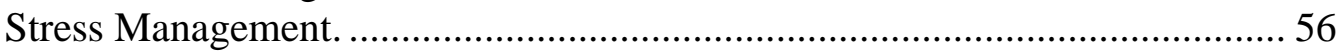

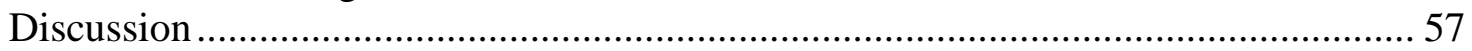

Implications for Occupational Therapy Practice ................................................... 61

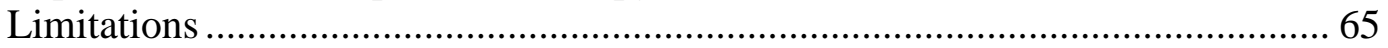

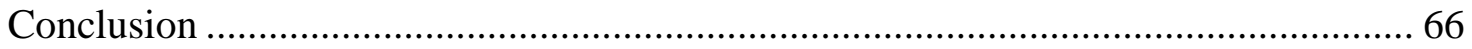

Chapter Four: Experiences of Veterans Transitioning into Academia .............................. 68

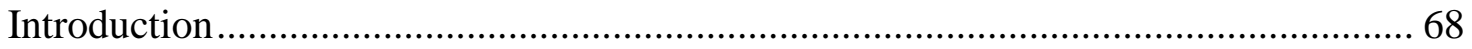

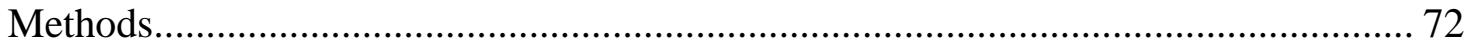

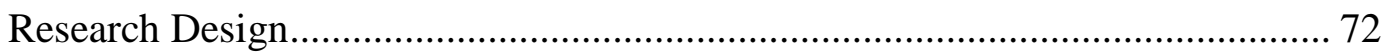

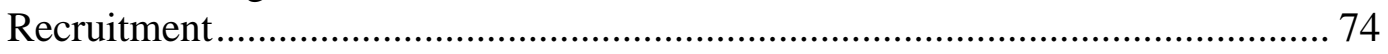

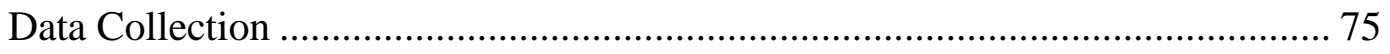

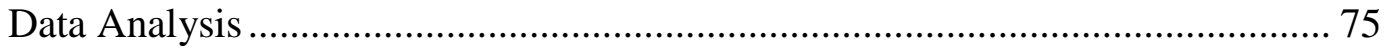

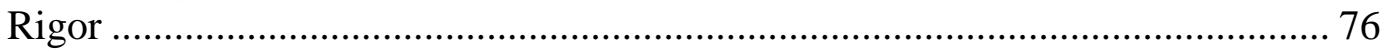

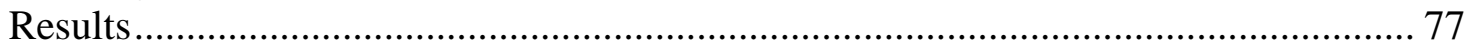

Theme 1: Repurposing Military Experiences for Civilian Life ........................... 80

Moving On From Military Frustrations ....................................................... 81

Finding Veterans Who Could Understand...................................................... 82

Theme 2: Reconstructing Civilian Identity ........................................................... 84

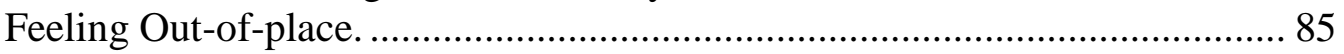

Projecting "Self" as a Professional. .................................................................. 87

Theme 3: Navigating Postsecondary Context and Interactions ............................ 88

Adapting to a New Context........................................................................ 88

Difficulty Relating to Students. .................................................................... 89

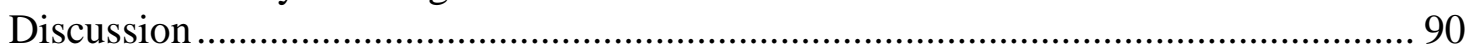

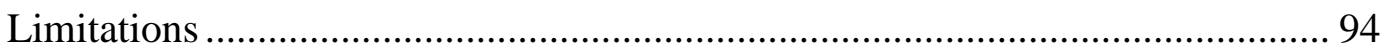

Implications for Occupational Therapy Practice ……………………………...... 95

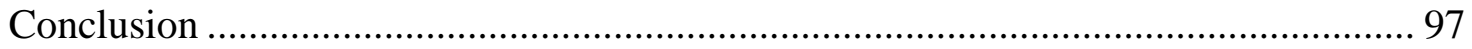

Chapter Five: Student Veteran Occupational Transitions in Postsecondary Education: A

Grounded Theory ........................................................................................... 99

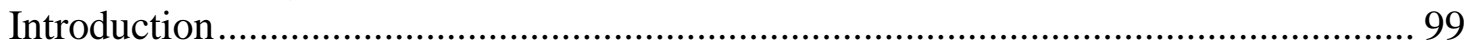

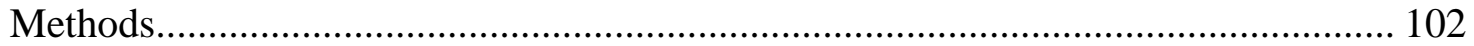

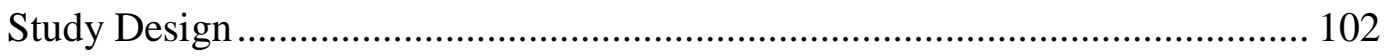




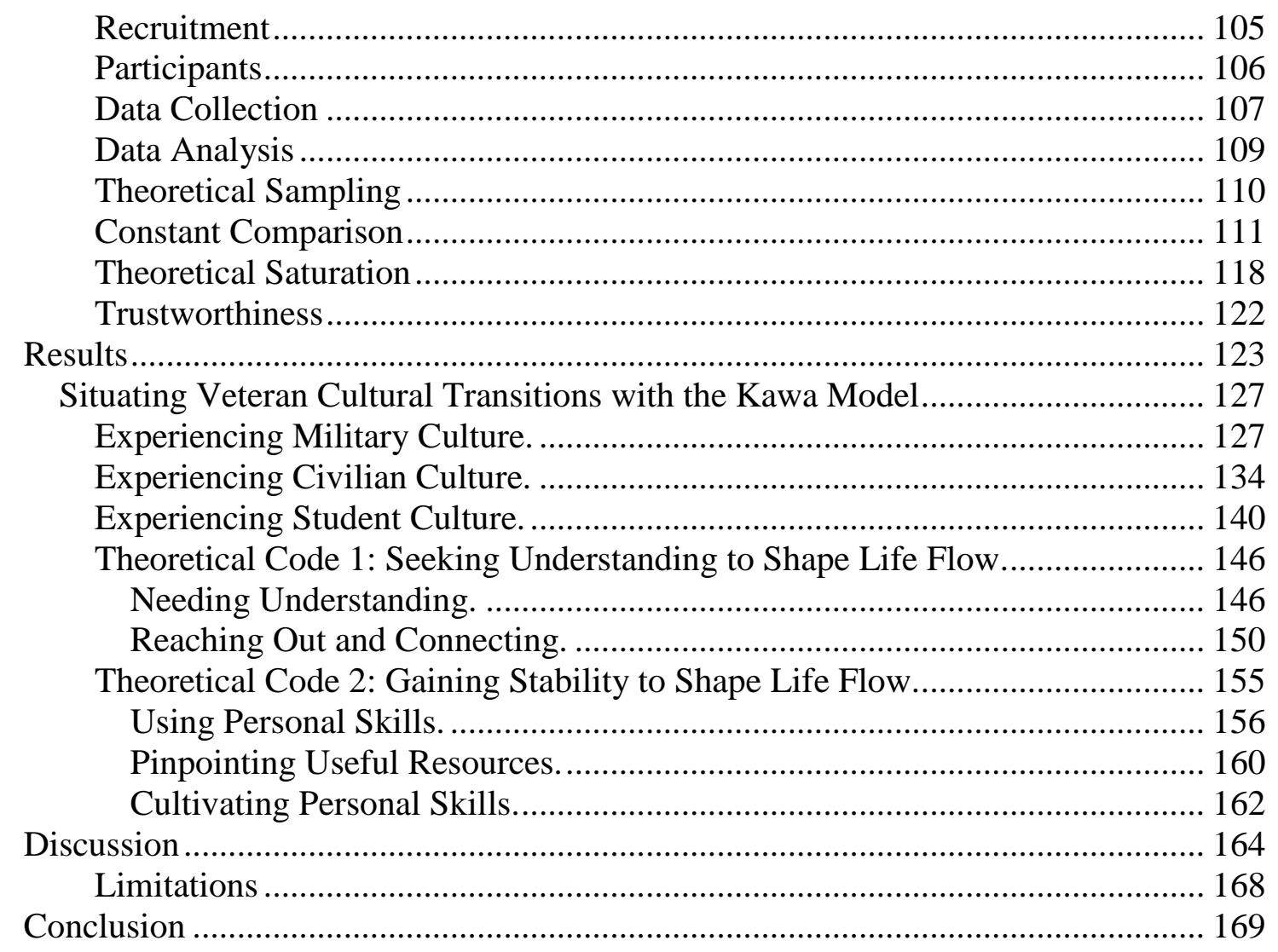

Chapter Six: Synthesis of Dissertation Results........................................................ 170

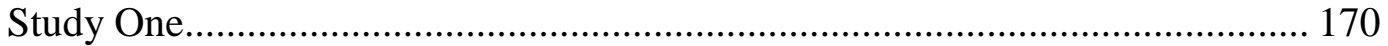

Study Two

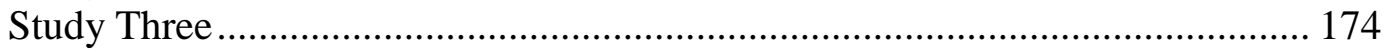

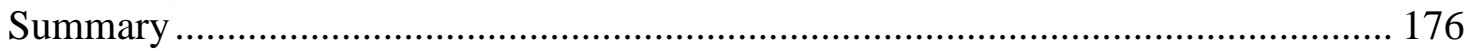

Implications for Practice ……………….......................................................... 177

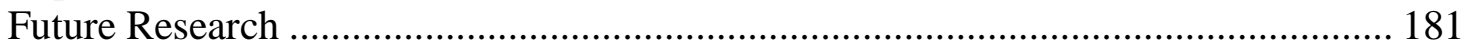

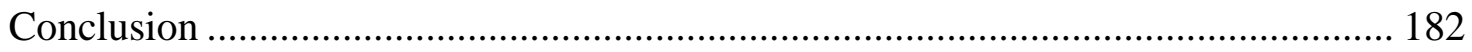

APPENDIX

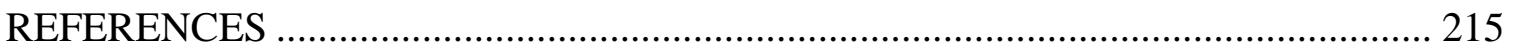

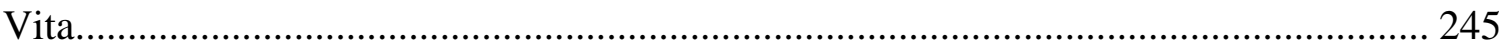




\section{LIST OF TABLES}

Table 3.1: Demographics of Respondents

Table 3.2: Coping Mechanisms Reported by Student Veterans Experiencing an Adult

Transition: Percentages.

Table 4.1: Grand Research Question, Sub-Research Questions, and Interview

Questions.

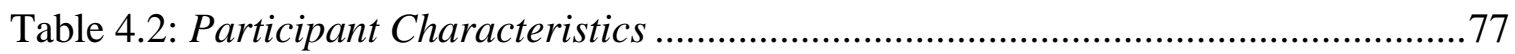

Table 4.3: Example Participant Statements for Themes and Sub-themes ........................79

Table 5.1: Kawa Model Features and Corresponding Contextual Representations .......108

Table 5.2: Examples of Initial Code Memos ...............................................................114

Table 5.3: Focused Codes and Operational Definitions ..............................................115

Table 5.4: Example of Focused Code Memo ..............................................................118

Table 5.5: Example of Theoretical code Memo ...........................................................120

Table 5.6: Constructivist Coding Process Summary ..................................................121

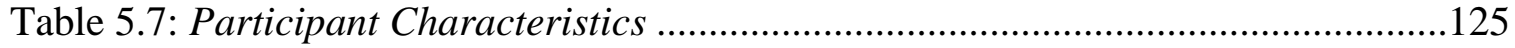




\section{LIST OF FIGURES}

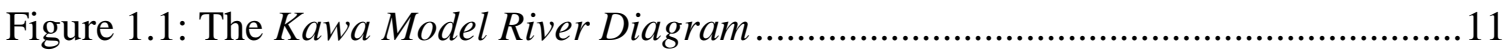

Figure 1.2: Cross-sectional View of the Kawa Model ..................................................12

Figure 3.1: Flow Chart for Respondents Included in Transition Survey Results ..............44

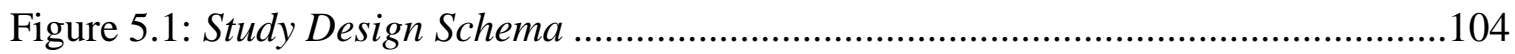

Figure 5.2: Cross-section of Collective Veteran Experiences in Military Culture ..........128

Figure 5.3: Cross-section of Collective Veteran Experiences in Civilian Culture ..........136

Figure 5.4: Cross-section of Collective Veteran Experiences in Student Culture ...........141

Figure 5.5: Emergent Model of Student Veteran Life Flow in Transition ......................147 


\section{Chapter One: An Introduction to Veteran Transitions}

Transitioning service members of the United States military have experienced the longest conflict since Vietnam with more than 2.2 million persons deployed to Iraq and Afghanistan (Institute of Medicine [IOM], 2013). Transition involves reintegration into the civilian community after active duty service, though the transition may not be "seamless" for veterans with combat service in Iraq and Afghanistan (Quillen-Armstrong, 2007; Stiglitz \& Blimes, 2008, p. 68). The transition is a complex process encompassing multiple dimensions that influence veteran perceptions of reintegration or re-entry into civilian life (MacLean et al, 2014). The majority of service members with combat exposure return to civilian life with minimal long-term problems, however, $44 \%$ report difficulties transitioning into civilian life (Morin, 2011) with the prevalence of readjustment problems such as social functioning, productivity, and self-care ranging from 25 to 54 percent (Sayer et al., 2010).

The harsh realities of combat injury and exposure to injured combatants drastically affects the transition experience for veterans of the second Gulf War era. Mortality following combat deployments to Iraq and Afghanistan during this era includes 4,425 in Operation Iraqi Freedom (OIF), 66 in Operation New Dawn (OND), and 2,355 in Operation Enduring Freedom (OEF), while morbidity accounts for over 52,000 total service members wounded-in-action for all three conflicts combined (United States Department of Defense, 2015). These casualty rates of military service members reflect the aggregate injuries and deaths incurred during wartime service, however, the longterm effects are unknown for veterans as they transition from military to civilian environments (IOM, 2013). One conservative estimate for long term financial costs 
reports $31 \%$ of veterans in the Gulf War II era have already filed for "service-connected disability," with the rate expected to increase similar to the rates (45\%) of the first Gulf War as the Department of Veterans Affairs processes through its historic backlog in disability claims (Stiglitz \& Blimes, 2008, pp. 76-77).

The etiology of Gulf War II era injuries have been mostly attributed to improvised explosive devices (IEDs) in unconventional warfare tactics (Kelty, 2010; Stiglitz \& Blimes, 2008, p. 82). Poly-traumatic injuries or multiple body and limb injuries sustained from IEDs blasts account for the primary surgical interventions performed in deployed environments (Gawande, 2004). Research has demonstrated that OEF/OIF veterans are more likely to survive war injuries sustained in this era compared to previous conflicts due to advances in medical practices (Clark, Scholten, Walker, \& Gironda, 2009; Gawande, 2004). In fact, the rate of survival following combat related injuries in the Gulf War II era has been reported at 90\% (Clark, Bair, Buckenmaier, Gironda, \& Walker, 2007). However, surviving blast injuries presents a conglomeration of long-term functional problems in the coalescence of co-morbidities. For instance, three common symptomologies such as chronic pain, post-traumatic stress disorder (PTSD), and post concussive symptoms formulate the polytrauma clinical triad for blast injury survivors (Lew, Otis, Tun, Kerns, Clark, \& Cifu, 2009). The symptoms of the polytrauma clinical triad and associated functional challenges are difficult to manage early in rehabilitation which necessitates close awareness and intervention as it complicates many veteran's readjustment to civilian life as a chronic disorder (Gironda et al., 2009).

Another major group of injuries affecting the transition of veterans are the "invisible wounds" incurred during combat such as cognitive dysfunction associated with 
traumatic brain injuries, and/or psychological and behavioral problems experienced by service members (Tanielian \& Jaycox, 2008). Traumatic brain injuries account for $22 \%$ of combat related disorders, whereas the prevalence of psychological injuries such as anxiety, post-traumatic stress disorder (PTSD), and depression following combat exposure is $30 \%$ for Gulf War II era veterans (Hoge et al., 2004; Hoge, Auchterlonie, \& Milliken, 2006; Hoge, Terhakopian, Castro; IOM, 2013; Messer \& Engel, 2007; Tanielian \& Jaycox, 2008). In a study of 50,184 service members, 40\% who reported combat exposure and were on active duty between 2001 and 2006, had three times the likelihood to experience PTSD symptoms or be diagnosed with PTSD (Smith et al., 2008). The hallmark injuries of the mind during this era of combat service highlights mental health practice areas of concern for occupational function as veterans transition to civilian life.

Similar concerns for Gulf War II era veterans reside in the repeated exposure to combat environments and the accompanying interpersonal conflicts. Repeated exposure to combat environments perpetuate a psychosocial strain on the overall military force and its family members (Betancourt, 2013; Eaton et al., 2008; Hoge, Auchterlonie, \& Milliken, 2006). Engaging in multiple deployments disrupts family routines, causes relationship demands, and presents family-oriented socioeconomic consequences as many service members report difficulty balancing these demands as the primary reason for leaving the military prematurely (AIR, 2005). The complex work demands and frequent separations affect family dynamics between children, spouses, and other service members (Cogan, 2014). Relationship distress is a particular concern for military populations as more than a third of completed suicides in the military are directly 
attributed to "relationship loss" with little influence attributed to the effect of deployment service or combat exposure (Bush et al., 2013). In fact partner relationship issues double a service member's odds of suicidality (Skopp et al., 2012). The concerns for suicidal ideations and attempts continue into civilian life via a disheartening statistic as the Department of Veteran Affairs reports 22 veterans commit suicide each day (Kemp \& Bossarte, 2013).

The high incidence and prevalence estimates of morbidity, mortality, and survivability for Gulf War II era veterans suggests that occupational therapists will likely encounter veterans in a myriad of contexts such as rehabilitation settings, community centers, or in educational institutions. Veterans from this war era transition to civilian life with numerous service-based entitlements afforded for "medical treatment, long-term health care, pensions, educational grants, housing assistance, reintegration assistance, and counseling" (Stiglitz \& Blimes, 2008, p. 64). The Post 9/11 GI Bill entitlement has made higher education more accessible for transitioning veterans and has illuminated an emergent context with a limited research base (Ackerman, DiRamio, \& Mitchell, 2009; Church, 2009; Clemens \& Milsom, 2008; Zinger \& Cohen, 2010).

Research studies on veteran transitions in the postsecondary education context is further sparse within the occupational therapy literature as only two investigations exist (Plach \& Sells, 2013; Tomar \& Stoffel; 2014). In the first study reviewed, Plach and Sells (2013) identified that 70\% of their sample studied experienced problems with transitioning to postsecondary education and ranked the occupation of education as a priority problem area when assessed with the Canadian Occupational Performance Measure. In a second study reviewed, Tommar and Stoffel (2014) examined the 
transition experiences of two student veterans in a Photovoice study (Wang \& Burris, 1997) that revealed themes of reminiscence of past duty, transition from military to civilian student life, entry to a new stage in life, and influences of the university and community context. In general, the occupational therapy literature is lacking a theoretical orientation for conceptualizing the experiences of student veterans. Further inquiry is needed to identify current transition strengths and liabilities that must be leveraged by occupational therapists working with student veterans.

\section{Statement of the Problem}

Transitioning veterans enrolled in four year institutions often fail to graduate or take longer to graduate as attrition rates range between 46-88\% (Cate, 2014; Wood, 2013). These rates highlight the need to support veterans in the occupation of education, however, occupational therapists require a deeper conceptualization of how to provide occupation-based support for student veterans. The first challenge lies in describing the attitudes and beliefs that influence how veterans cope with their transition from the military to postsecondary education context. The second challenge lies in understanding the influence of veterans' lived experiences as they transition into the postsecondary educational context. The last challenge lies in understanding the individual and collective constructions of student veteran transitioning experiences as it relates to culture, time, and context.

\section{Statement of Purpose and Research Questions}

The purpose of this three-part dissertation was to explore the transition experiences of student veterans reintegrating into the civilian context of postsecondary education following an honorable military career and history of military overseas 
deployment. We anticipated that examining the transition experiences through a culturally relevant lens, would elicit a deeper understanding of the factors that influence the transition process and provide key areas in which therapists may promote occupationbased practice to support student veteran transitions. In order to address these problems, we investigated the following research inquiries:

\section{Study 1 Specific Aims}

Aim 1: Identify intrinsic and extrinsic factors perceived as barriers and/or supports for transitioning to postsecondary education.

Aim 2: Identify the critical needs of student veterans consistent with the domain of the occupational therapy practice.

Given these two aims, we developed and pilot tested a survey instrument to quantitatively assess military to civilian coping responses in a cross-section of student veterans enrolled at a single, large land grant university. From the survey responses we were able to 1) describe how student veterans appraised their transition, 2) describe which methods of coping were most frequently reported, and 3) identify individual and environmental factors that required support from occupational therapists and rehabilitation practitioners.

\section{Study 2 Specific Aim}

Aim 1: To answer the question, "What are the lived experiences of discharged military veterans transitioning to the occupation of higher learning at a single large land grant university?" We decided to qualitatively interview student veterans, following study one, in order to reach a larger sample and attain a deeper understanding of their lived experiences in transitioning. From the aim we were able to understand 1) veterans' 
attitudes when transitioning to academia, 2) the reasons veterans report using their Post 9/11 GI Bill, 3) social support used by veterans transitioning to academia, 4) veterans' attitudes towards the military as they transition to academia, and 5) veterans perspectives on relationships with their peers at the university.

\section{Study 3 Specific Aim}

Aim 1: To answer the grand tour question, "What is the meaning of the transition experience to postsecondary education for student veterans?" Given the development of themes categorizing student veteran transition experiences in study two, we decided to research student veteran transition experiences in relation to postsecondary education culture and context using the qualitative grounded theory methodology. This method was used to raise the descriptive themes of study two into a constructed theoretical conceptualization for student veteran transitions. From this aim we were able to explain, 1) how student veterans describe their current physical and social environment, 2) the life circumstances that shape student veteran transitions to postsecondary education, and 3) how individual assets and liabilities influence student veteran transitions to postsecondary education.

\section{Research Approach}

Chapter three describes the methodology and results for study one. Study one was a cross-sectional study utilizing a Tailored Survey design to collect structured, anonymous survey data from veteran respondents attending a large land grant university. The Tailored Survey method is a scientific research design that reduces four principles of survey error: coverage, sampling, nonresponse and measurement (Dillman et al, 2009). We chose this design as it established a set of procedures for the sampled participants to 
follow online and encouraged all respondents to complete the electronic mail survey as a positive social exchange.

Chapter four describes the methodology and results for study two. Study two employed a qualitative, transcendental approach to phenomenology, which focused on naming themes from rich textual descriptions directly quoted by participants engaged in semi-structured interviews. A qualitative phenomenological study design was chosen as it described the lived human experiences based on the principle that "scientific knowledge begins with unbiased descriptions of the subject matter" (Polkinghorne, 2005; Wertz, 2005). The complex interplay of veterans' personal subjective experiences could only be interpreted within a qualitative study design. Furthermore, the qualitative phenomenological research design empowered participants to share their stories and voices on experiences and contextual factors considered unanalyzable by conventional statistical methodologies (Creswell, 2013).

Chapter five describes the methodology and results for the final dissertation study. We employed a Constructionist Grounded Theory (CGT) approach described by Charmaz (2006) using the Kawa (Japanese for River) model "a priori” as a culturally relevant guide for interviewer-participant discussions (Iwama, 2006). CGT acknowledges the multiple realties research participants experience, in which researchers use, to construct an abstract theory interpretive of the social interactions between both the researcher and participant (Charmaz, 2006, pp. 9-12). Use of the grounded theory methodology surpassed a description of the experiences of participants highlighted in studies one and two and focused specifically on generating an abstract model that conceptualized the experiences of the participants (Creswell, 2013). Thus, qualitative 
data was collected through individual, intensive researcher/participant interviews, participant Kawa diagram drawings, researcher reflective journaling, and member checking interviews. Consistent with the grounded theory tradition, data collection and analysis was performed simultaneously (Charmaz, 2006, pp. 178-80). However, the focus centered on developing a middle range theory without forcing data into pre-existing categories and explicitly acknowledging its construction as a product of the interactions between participants and researchers (Charmaz, 2006, p. 119).

\section{Theoretical Framework: The Kawa (Japanese for River) Model}

The Kawa model described by Iwama (2006) served as a novel occupational therapy focused, culturally relevant tool for guiding participant inquiry. The Kawa model operationalizes the construct of culture as the "shared experiences and common spheres of meaning" to include "the collective social processes by which distinctions, meanings, categorizations of objects and phenomena are created and maintained" (Iwama, 2006, p.8). Researchers applying this model assume participants develop their worldview from a multitude of dynamic factors such as assigning meaning to objects, developing attachments within the environment, and relating experiences to existing temporal factors. The construction of meaning is assumed to vary between each person given their individualized interactions within an established environmental social frame. The Kawa model highlights differences between Western and Eastern views of agency, or the capacity to act independently (Nahab et al., 2011), as the Japanese culture from which the model derives operates within a collectivistic social frame or hierarchy.

Military culture resembles Eastern cultures as service members establish interdependence within the social environment and focus on collective aspirations for 
constructing meaning. According to Iwama et al. (2009), relationships are coveted in collectivistic social frames as they enhance experiences of "belonging" and “interdependence." Military service members report being isolated and separated from these relationships and social networks when they transition into civilian culture as a veteran (Koenig et al., 2014). Furthermore, veterans develop their social identities specifically from a military environment which requires support when transitioning to the civilian context (Demers, 2011; Koenig et al., 2014). Therefore, a culturally relevant model of occupational therapy, such as the Kawa, served well as a guiding lens for examining the experiences of veteran culture.

The Kawa model uses the metaphor of a river to represent the life flow of a client, identifying areas for occupational therapists to intervene and promote harmony to balance the client within their ascribed context (Humbert, Bess, \& Mowery, 2013; Iwama, 2006; Iwama, 2007; Iwama et al., 2009; Munoz, 2007; Paxson et al., 2012; Wada, 2011). Iwama (2006, 2009) describes five components of the model as follows: water representing life flow, river side-walls and bottom representing the physical and social environment, rocks representing life circumstances, driftwood representing a person's assets and liabilities, and spaces representing the areas in which occupational therapy can support the client. Figure 1.1 and 1.2 provides an example diagram of the Kawa model attributes. 
Figure 1.1 The Kawa Model River Diagram

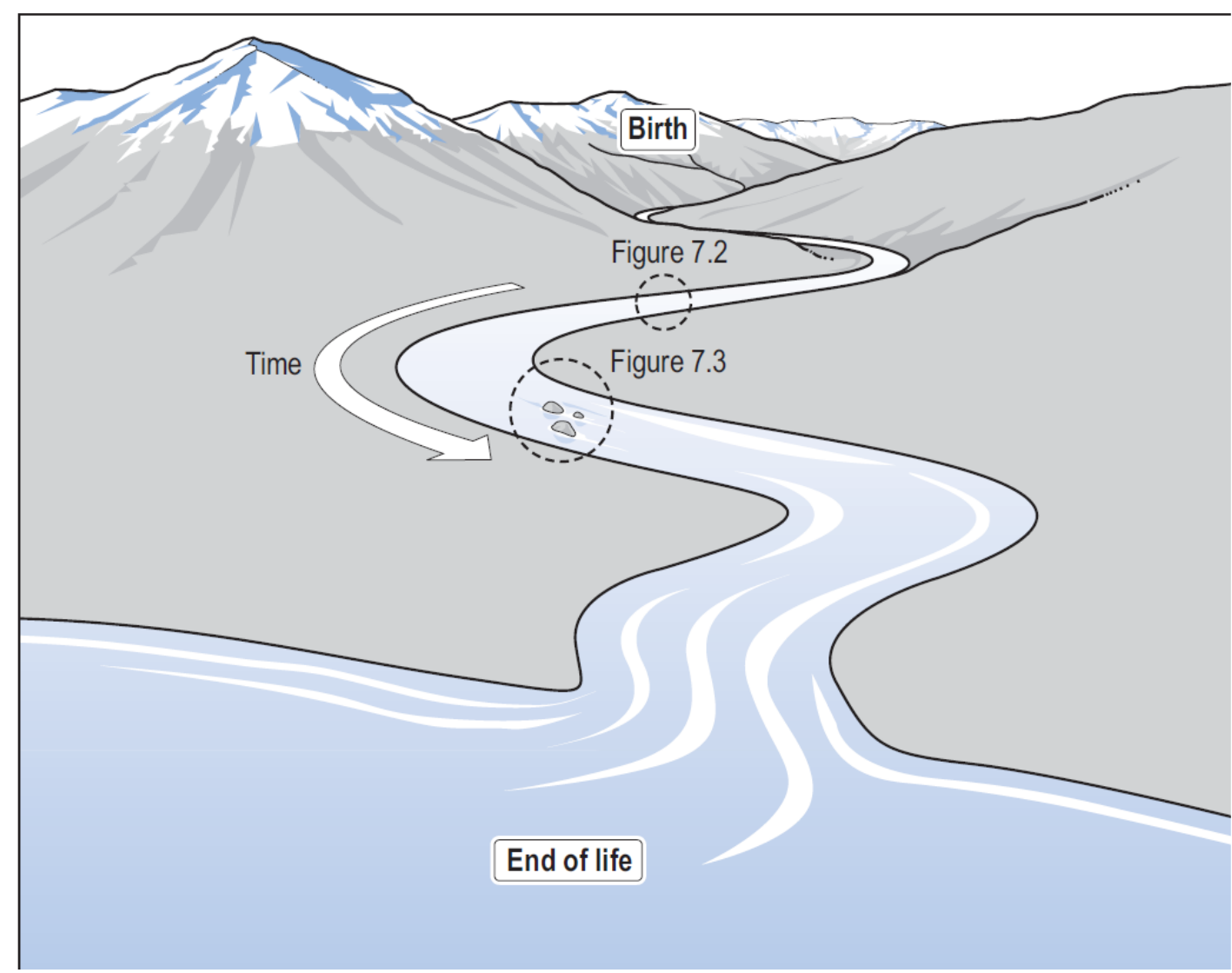

Reprinted from The Kawa Model: Culturally Relevant Occupational Therapy, Iwama, M. K., An Overview of the Kawa Model, Figure 7.1, p. 143, (2006) with permission from Elsevier. 
Figure 1.2 Cross-sectional View of the Kawa Model

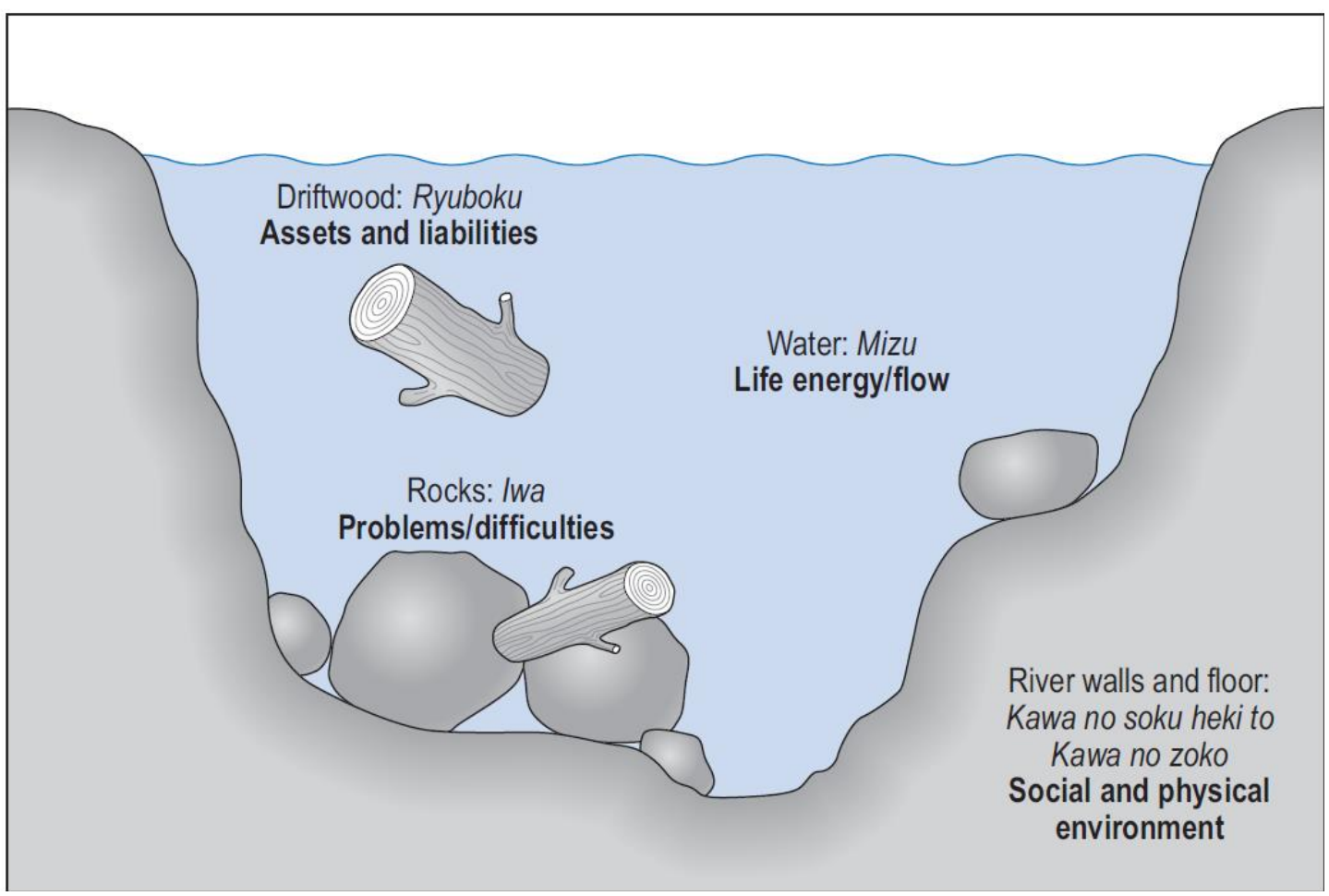

Reprinted from The Kawa Model: Culturally Relevant Occupational Therapy, Iwama, M. K., Raising a New, Culturally Relevant Conceptual model of Occupational Therapy from Practice, Figure 6.5, p. 132, (2006) with permission from Elsevier. 
The Kawa model was also selected due to its epistemological underpinnings in social constructionism. Charmaz (2006, p. 189) describes social constructionism as a worldview in which people construct social realties through individual and collective actions. The incorporation of the Kawa model aligned well with CGT due to the shared philosophical underpinnings of constructionism. The union of these two approaches enhanced the process for conducting intensive interviews. The intensive interviews facilitated in study three were designed to elicit participant descriptions and reflections on their experiences in methods that seldom occur in daily life (Charmaz, 2006, p. 25) which was also consistent with the use of the Kawa model.

\section{Assumptions}

I have held four primary assumptions prior to data collection for each study. These assumptions originated from my clinical and professional experience, continuing education, and doctoral research studies. The first assumption I held was that veterans experienced a series of readjustment challenges when leaving the military and enrolling in higher education. This assumption was based on my experiences working with military service members who had participated in the military transition assistance program and from speaking with veterans enrolled at the local university I attended. The second assumption I carried was that participants would be able to verbalize experiences of both supports and challenges related to transitioning to the university. This assumption was based on my preliminary social interactions and in-depth conversations with student veterans on campus, local university veteran office directors, and university administrators working with veterans. The third assumption I carried was that participants would provide honest and truthful answers to the participant demographic 
form and their military deployment history. The last assumption related to my final study. I assumed veterans, "sustain knowledge by social processes and that knowledge and social action go together" for a collective social focus (Young \& Collin, 2004). In this perspective, I adopted a social constructionism epistemological lens for interpreting the transition experiences of my research participants (Gergan, 2001b), as I considered their transition experiences to be a product of collective processes versus completely individualized actions.

\section{Researcher Statement}

The time period of these studies encompassed research and completed coursework within the Rehabilitation Sciences Ph.D. program at the University of Kentucky. The rehabilitation science coursework and program led me down a pathway towards qualitative research on the needs and experiences of military veterans, however, I retain numerous experiences and attachments to military culture further compelling the research line that I have conducted. I am an active duty Army occupational therapist with a 10 year history of service to military service members, dependents, and veterans. I have military medical experiences in physical disability rehabilitation, orthopedic hand therapy, mental health, clinical administration, and medical detachment command. I was competitively selected for an Army educational funding program to complete my doctoral studies and was most humbly, accepted to attend the University of Kentucky's Rehabilitation Science program.

My research interest in military transition needs stemmed from my experiences of commanding an Army Combat Support Hospital (CSH) rear detachment. This assignment placed me in charge of 87 Soldiers, with a high proportion subject to a variety 
of injuries, disabilities, co-morbidities, substance abuse issues, and professional conduct problems. Though there were high rates of these challenges that routinely lead to separation from military service, the majority of the Soldiers expressed a strong desire to continue serving their country in spite of their current life circumstances. My role was to provide leadership for all of my subordinates in performing our stateside mission and simultaneously preparing four other medical detachments for overseas deployments. I learned firsthand from these experiences the necessity in assessing both risk and protective factors of Soldiers' health and well-being. I also learned how important it was to place Soldiers in meaningful occupations that leveraged each Soldier's capability for optimal performance. We placed many Soldiers in positions atypical for their intended military occupational specialty, however, the assignments were made based on our unit needs and on the assessed strengths of each Soldier. These actions could only work with a supportive environment, motivated Soldiers, and continuous education and training. Fortunately, we were quite successful in ensuring each medical detachment we were responsible for was properly trained, well equipped, and highly prepared to perform their overseas mission.

It was my mission to ensure each Soldier had a purpose and professional meaning attached to each task they were assigned to perform. However, the reality of a rear detachment is that you are charged with separating Soldiers from the military, which poses many dilemmas. One of the major dilemmas I often wrestled with, were thoughts of how Soldiers would occupy themselves in civilian life. Were they prepared for life after the military and did they have a plan for their future? Growing up in a military family and raised in a military community, I had observed the challenges many veterans 
face upon leaving the military. I witnessed numerous instances of veterans with difficulty attaining work due to narrow military professional skills developed in low demand career fields. I also witnessed the difficulty many veterans experience with sustaining work due to numerous injuries incurred that limited functional performance for the civilian job demands. With these recollections and vivid images emblazoned in my mind, I always tried to support the educational requests of my Soldiers once I joined the military. I wanted to ensure that Soldiers reinvested in their skill sets with the entitlements that they had earned.

The research I have conducted in my doctoral studies has provided a deeper understanding on the impact of transitioning to civilian life following military service. The research that I have conducted has provided a deeper description of the challenges veterans face during a period of prolonged conflict. What makes this era of service members unique, following fourteen years at war, is the global access to higher education. The Post 9/11 GI Bill is providing an opportunity to veterans and they are taking heed in large cohorts. So, as veterans develop skills for sustaining a civilian career, the nation must support a growing population of non-traditional students that have voluntarily experienced the realities of war, injury, disability and recovery as they return to civilian communities. These realities beckon a holistic and culturally relevant lens for understanding the needs of student veterans. My primary goal is to empower veterans to develop and share their own narratives with the professionals devoted to supporting their needs. The research conducted was intended to identify supportive processes for veterans and to diminish any barriers towards their success following a career of honorable service. Ultimately, my big picture, 30,000 foot view with the research questions that I 
have investigated is to navigate practitioners toward the delivery of compassionate and competent occupational therapy services for the American veteran.

\section{Significance of the Study}

Veterans are entering educational institutions at higher rates than previous eras with the use of the Post 9/11 GI Bill and due to the systematic downsizing of the military. In a Department of Veteran Affairs report, veteran enrollments into postsecondary education had increased by $42 \%$ with over 900,000 enrollments reported from 2009-2012 (Department of Veterans Affairs, 2014). The growth of the student veteran population is a particular concern for rehabilitation practitioners as $44 \%$ of veterans report difficulty transitioning due to psychosocial and disability related factors that predict a difficult transition (Morin, 2011). The salience of psychosocial and disability related factors suggest that rehabilitation practitioners will likely engage veterans of the Post 9/11 era in a clinical or community-based setting whom express interest in enrolling into postsecondary education. Furthermore, rehabilitation practitioners must be prepared for the growing population of veterans transitioning to civilian life and primarily, to postsecondary education. Veterans will want to develop their skills for civilian living regardless of the their current life circumstances yet, we know that veterans take longer to graduate (McBain et al., 2012) and fall victim to academic attrition at higher rates than traditional age students (Cate, 2014; Wood, 2013).

The educational environment provides a novel context for rehabilitation practitioners working primarily, within a medical institution. The limited rehabilitation and occupational therapy literature available describing student veteran transition experiences, highlights the importance of these studies. Therefore, the studies conducted 
within this dissertation add towards the modest body of literature which examines the transition needs of student veterans. It also adds an emergent conceptual understanding of transition process within the perspective of life flow. In the first study, surveying the attitudes and beliefs of student veterans coping with a transition to academia will expand therapists understanding of individualized strategies and environmental supports used to enhance their transition experiences. In the second study, learning about the lived experiences of student veteran transitions will guide clinicians in understanding what occupational areas must be supported to enhance the transition to the postsecondary education context. Finally, the third study will help guide occupational therapy practitioners and educators on the transition process and to the domains in which occupational therapy can best apply a culturally relevant approach to guiding student veteran transitions.

\section{Delimitations}

1. Participants were males and females between the ages of 20 and 45 .

2. Participants in the third study were delimited to reporting a history of military deployment.

3. Participants were veterans currently enrolled, recently graduated (within the past 2 years), or recently dis-enrolled (within the past 2 years) at the university.

\section{Limitations}

The studies were conducted at two educational institutions in a city of the Southeastern United States; therefore the results of this dissertation may only be generalized to those groups of individuals with similar characteristics to this sample. Participants were recruited from two universities with on-campus veteran resource 
centers or offices. So, veteran experiences in this sample may differ from those of veterans attending institutions with more limited veteran supports. Also, the majority of the student veterans interviewed within this study were Caucasian, but differences in transition experiences may exist across veterans of differing racial and/or ethnic groups.

\section{Operational Definitions}

Coding: is defined as, "the process of defining what the data are about. Unlike quantitative grounded theorist creates qualitative codes by defining what he or she sees in the data" (Charmaz, 2006, pp. 186-187).

Constant Comparative Method: is defined as, "a method of analysis that generates successively more abstract concepts and theories through inductive processes of comparing data with data, data with category, category with category, and category with concept. Comparisons then constitute each stage of analytic development" (Charmaz, 2006, p. 187).

Constructivism: is defined as, "a social science perspective that addresses how realities are made. This perspective assumes that people, including researchers, construct the realities in which they participate. Constructivist inquiry starts with the experience and asks how members construct it... Constructivists acknowledge that their interpretation of the studied phenomenon is itself a construction" (Charmaz, 2006, p. 187).

Constructivist Grounded Theory: is defined as, "a constructivist approach that places priority on the phenomena of study and sees both data and analysis as created from shared experiences and relationships with participants and other sources of data" (Charmaz, 2006, p. 133). 
Culture: is defined in a broader view, "as shared spheres of experience and meaning, rather than the individually bound definitions that treat culture as static embodiments like ethnicity and race. A culture may consist of a particular age group, geographical location, religious orientation... any group of people who share common experiences and meanings of such experiences" (Iwama, 2006, pp. 105-106).

Harmony: is defined as, "a state of individual or collective being in which the subject, be it self or community, is in balance with the surrounding context" (Iwama, 2005, p. 217).

Invisible Wounds: is defined as the, "mental health conditions and cognitive impairments resulting from deployment experiences" (Tanielian \& Jaycox, 2008, p. 22). Kawa Model: the Kawa model described by Iwama (2006) provides a visual graphic/illustration of the client's subjective experience under inquiry with the use of a river metaphor. The Kawa model serves as a novel occupational therapy focused, culturally relevant tool for guiding participant inquiry. Furthermore, "The Kawa model attempts to explain occupational therapy's overall purpose, strategies for interpreting a client's circumstances and clarify the rationale and application of occupational therapy within the client's particular social and cultural context" (Iwama, 2006, p. 139).

Life Flow: is defined as the abstract conceptualization or overall, "essence of harmony... Occupational therapy's purpose is to help the subject enhance and balance this flow" (Iwama, 2005, p. 217).

Major Depressive Disorder: is a depressive disorder that is, "characterized by discrete episodes of at least 2 weeks' duration (although most episodes last considerably longer) involving clear-cut changes in affect, cognition, and neurovegetative functions and interepisode remissions" (American Psychiatric Association, 2013, p. 155). 
Occupation: is defined as, "to the daily life activities in which people engage.

Occupations occur in context and are influenced by the interplay among client factors, performance skills, and performance patterns. Occupations occur over time; have purpose, meaning, and perceived utility to the client; and can be observed by others (e.g., preparing a meal) or be known only to the person involved" (American Occupational Therapy Association, 2014).

Post 9/11 GI Bill: the Department of Veteran Affairs (2009b) defines the Post 9/11 GI Bill as, "an educational assistance program is established for individuals who served on active duty after September 10, 2001. This educational assistance program is effective August 1, 2009." To be eligible for the entitlement the active duty service member serves a minimum of 90 aggregate days excluding entry level and skill training (to determine when entry level and skill training may be included in the total creditable length of service) or serves a minimum of 30 continuous days and, after completion of such service, is discharged under other than dishonorable conditions due to a serviceconnected disability (Department of Veteran Affairs, 2009b).

Post-Traumatic Stress Disorder (PTSD): PTSD is defined as a trauma and stressorrelated disorder in, "which exposure to a traumatic or stressful event is listed explicitly as a diagnostic criterion. A diagnosis of PTSD includes the following criteria: (1) exposure to actual or threatened death, serious injury, or sexual violence, (2) presence of 1 or more intrusive symptoms (i.e distressing memories, dreams, or flashbacks), (3) persistent avoidance of stimuli associated with the traumatic event, (4) negative alterations in cognitions and moods associated with the traumatic event, (5) marked alterations in 
arousal and reactivity associated with the traumatic event, and (6) the duration of the disturbance" (American Psychiatric Association, 2013, p. 265-280).

Recovery: is defined as, "a process of change through which individuals improve their health and wellness, live a self-directed life, and strive to reach their full potential" (Substance Abuse and Mental Health Services Administration, 2012).

Social Constructionism: is defined as, "a theoretical perspective that assumes that people create social reality(ies) through individual and collective actions. Rather than seeing the world as given, constructionists ask, how is it accomplished? Thus, instead of assuming realities in an external world-including global structures and local culturessocial constructionists study what people at a particular time and place take as real, how they construct their views and actions" (Charmaz, 2006, p. 189).

Theoretical Sampling: is defined as, "a type of grounded theory sampling in which the researcher aims to develop the properties of his or her developing categories or theory, not to sample randomly selected populations or to sample representative distributions of a particular population. When engaging in theoretical sampling, the researcher seeks people, events, or information to illuminate and define the boundaries and relevance of the categories" (Charmaz, 2006, p. 189).

Theoretical Saturation: is defined as, "the point at which gathering more data about a theoretical category reveals no new properties nor yields any further theoretical insights about the emerging grounded theory" (Charmaz, 2006, p. 189).

Transition: is defined as a process that involves veterans', "reintegration into the civilian community after active duty service (Stiglitz \& Blimes, 2008, p. 68). The transition is 
examined as a complex process encompassing multiple dimensions that influence veteran perceptions of reintegration or re-entry into civilian life.

Veteran: the Veteran Benefits Act (1945) defines a veteran as, "a person who served in the active military, naval, or air service, and who was discharged or released therefrom under conditions other than dishonorable."

\section{Summary}

The transition needs of veterans is a priority for healthcare practitioners and likewise, the occupational therapy profession. Veterans' needs must be richly described in order to illuminate the complexity of their challenges and to identify how occupation may best facilitate a positive, healthy, and productive transition. There is an acute need to better understand veteran transitions to postsecondary education, and to create supportive environments that guide each veteran harmoniously within their individualized and collective life flow.

\section{Portions of this chapter pertaining to the Kawa Model have been previously published:}

Gregg, B.T., Howell, D.M., Quick, C.D., \& Iwama, M.K. (2015). The Kawa river model: Applying theory to develop interventions for combat and operational stress control. Occupational Therapy in Mental Health, 31(4), 366-384. DOI:10.1080/0164212X.2015.1075453

Copyright (C Brian Tuan Gregg 2016 


\section{Chapter Two: Review of the Literature \\ Introduction}

The focus of this chapter critically reviews contemporary literature regarding veteran transitions to civilian life. Peer-reviewed articles were systematically searched using the databases of Medline, BIDS SCI and SSCI, PUBMed, PsycINFO, CINAHL, Google Scholar, OT Search, and the Cochrane Library. The review identified qualitative and quantitative studies published between 2000 and 2015, in the English language. Due to the wide variance in methodologies and research problems examined by each paper, this literature review has been categorized into broad research content areas inclusive of veteran transitions to civilian life. Following this introduction, three major veteran transition content areas are described: Measurement of Veteran Transition Challenges, Veteran Transitions into Gainful Employment, and Veteran Transitions into Postsecondary Education. The review adds a discussion on the results of the literature review and provides implications from an occupational science perspective.

\section{Measurement of Veteran Transition Challenges}

There appears to be a limitation on the amount of studies that measure the difficulties experienced by veterans transitioning to civilian life. However, one study was conducted by Morin (2011) in which 1,853 veterans were surveyed on their reentry into civilian life. Survey items were constructed following an extensive review of previous qualitative and quantitative studies covering a broad array of veteran problems when leaving the military. When analyzing the entirety of the veteran responses, most veterans (72\%) reported an easy time readjusting to civilian life though the proportion of veterans reporting difficulty increases (27\% to $44 \%$ ) when stratifying responses from Post 9/11 
Veterans (Morin, 2011). Furthermore, six of the eighteen factors experienced during their military service predicted a more difficult veteran re-entry into civilian life: (1) experiencing a traumatic event while serving, (2) being seriously injured while serving, (3) Post-9/11 veteran who was married while serving, (4) being a Post 9/11 veteran, (5) serving in combat, and (6) knew someone killed/injured while serving (Morin, 2011).

The transition concerns highlight the perceived impact physical and psychosocial injuries may have on veterans and suggests the importance of medical and social support during the transition into civilian life. Sayer et al. (2010) conducted a study that supports the concerns in social functioning and participation veterans experience in their prevalence study on community reintegration problems among 754 Gulf War II veterans receiving VA medical care. They reported 25 to 56 percent of veterans experienced difficulty in the areas of social functioning, productivity, community involvement, and self-care. Fifty-seven percent of the veterans in the study reported anger issues and thirty-one percent reported increased alcohol and drug use one year after reintegration to civilian life (Sayer et al., 2010).

In their efforts to develop a reliable and valid instrument for measuring community reintegration for service members, Resnik, Gray, and Borgia (2011) tested the psychometric properties of a novel veteran specific measure known as the Community Reintegration of Service members (CRIS) in a pretest (visit 1), posttest (3 months) study design. The researchers tested 68 seriously injured combat veterans in receipt of rehabilitation services at the military Center for the Intrepid (CFI) comparing differences in wounded service members with and without PTSD, TBI, and depression. The researchers demonstrated the CRIS to be a reliable and valid instrument for measuring 
the community reintegration of wounded service members and equally responsive to changes as compared to the Quality of Life Scale (QOLS) and Short Form 36 for Veterans (SF-36V) with a mean detectable change (MDC) range of 17.4 to 23.2 for the subscales tested. Veterans with PTSD, TBI, and depression demonstrated lower group differences $(\mathrm{P}<.05)$ on all three (extent of participation, perceived limitations, and satisfaction with participation) of the CRIS subscales (Resnick, Gray, \& Borgia, 2011). Resnick, Tian, Ni, and Jette (2012) adapted the CRIS item pool for a second study to apply item response theory and Computer Adaptive Testing. In this study, the instrument was condensed and demonstrated "acceptable item fit" following exploratory and confirmatory analyses in a convenience sample of 517 veterans reintegrating into the community.

Larsen and Norman (2014) examined predictors of functional problems such as unlawful behavior, financial problems, and work-related problems for Gulf War II veterans reintegrating into civilian life. PTSD symptoms predicted problems in all functional domains assessed with increased risk ranging from 1.5 to 3.5 times in all domains except unlawful behavior. A positive alcohol screening increased the risk of engaging in unlawful behavior twofold at pre-separation (baseline) and fourfold at the one year assessment suggesting the need to assess for alcohol use and overuse in veterans (Larson \& Norman, 2014).

These studies demonstrate the complexity of veteran transitions and the likely, high variance associated with developing a responsive overarching assessment tool for measuring veteran transitions. They also demonstrate the paucity of studies available that measure veteran transitions to civilian life. These studies highlight the need to focus 
further empirical research in measuring common problems experienced by specific veteran groups transitioning to civilian life such as veterans transitioning with specific diagnoses. It also highlights the need of focusing research within the specific contexts to which veterans transition. In the literature, there exists studies consisting of veterans transitioning to civilian employment and postsecondary education.

\section{Veteran Transitions into Gainful Employment}

Gulf War II era veterans are affected by high levels of unemployment and underemployment compared to previous eras. The Institute of Medicine (2013) reported unemployment rates for post 9/11 veterans 18 years old and older were a third higher than matched non-veterans (12.\% with $8.7 \%)$ and almost two times higher when comparing the $18-24$ year old groups (30.2\% with $16.1 \%)$. Many factors may precipitate the high rates though some authors have suggested that the transition process impairs the creation of civilian networks needed to facilitate civilian employment opportunities (Clemens \& Milsom, 2008; Demers, 2011). Civilian networks provide support structures significantly needed for veterans that often seek out their first civilian vocation following military service (IOM, 2013).

Temporal factors such as the generalized economic downfall in the U.S. from 2008-2012 have limited opportunities for veteran employment as Church (2009) notes, the recession led to the "largest level of jobless claims in the United States since the fall of 1982" (p. 50). The significance of the recession cannot be overlooked as veterans tend to occupy more traditional manual labor based vocations such as manufacturing, construction, and transportation then their non-veteran cohorts (U.S. Census Bureau, 
2010). Competition for these job markets are concerning for veterans and may precipitate social withdrawal and self-depreciative behaviors.

Bullock, Braud, Andrew, and Phillips (2009) examined the vocational concerns of combat veterans and correlates to career interests, personality, career thoughts, and career barriers. Participants $(n=55)$ receiving vocational rehabilitation services in a residential facility were enrolled in the study and administered four self-assessments: the Career Thoughts Inventory, the Career Attitudes and Strategies, the International Personality Item Pool, and the Self-Directed Search. The researchers concluded that negative career thoughts decrease emotional stability, job satisfaction, openness, and extraversion; increases in career worries and interpersonal abuse were also identified (Bullock et al., 2009). The negative thoughts and affirmations of veterans studied by Bullock et al. highlighted concerns expressed from homeless and unemployed veterans, whereas Haynie and Shepard (2009) studied wounded Marines in transition and the reconstruction of their identities through employment.

In the multiple case study by Haynie and Shepard (2010), 10 disabled Marines were interviewed on their transition perceptions following a career-retraining program in entrepreneurship. The participants attributed the traumatic injuries they sustained to the loss of their military careers, which negatively influenced their ability to form a new identity (Haynie \& Shepherd, 2010). The researchers suggested that participants used their career to determine a conception of self, whereas the termination of that career threatened the participant's self-identity.

Erbes, Kaler, Schult, Polusny, and Arbisi (2011) studied 262 National Guard/Reserve service members returning from OIF following a 16-month deployment to 
assess occupational functioning and predictors of employment rates. Diagnostic interviews and survey instruments were administered at baseline and 1 year after the initial assessment. The researcher reported 5\% had PTSD, 6\% had subthreshold PTSD, $11 \%$ had major depressive disorders, and $11 \%$ were experiencing alcohol abuse or dependence. The study found that the rates of employment of the study participants at both time points did not differ between veterans with and without a psychiatric diagnosis, however, those with depression, alcohol abuse, or PTSD reported lower work role functioning (Erbes et al., 2011).

Burnett-Ziegler et al. (2011) examined correlations between alcohol use, combat exposure, PTSD symptoms, and the number of deployments influencing employment status. National Guard service members $(n=585)$ participated during mandatory reintegration workshops and performed a battery of assessments. The researchers found that those with recent combat exposure (46\%) where more likely employed and that physical and mental symptoms did not affect employment status. However, those below the age of 30 were more likely to be unemployed (29\% with $57 \%$ ) compared to those 31 years of age or older. The researchers concluded that younger, low income veterans are less likely to be employed and mental health symptoms may not be related to initiating employment, rather it hinders sustaining employment (Burnett-Ziegler et al., 2011).

These studies identify key points in the employment concerns for transitioning veterans with disability, young age, and low income highlighting areas requiring support to enhance veteran occupational performance. As Elbogen et al. (2012) concluded in their study of financial wellbeing in over 1,388 Iraq and Afghanistan War veterans, "regardless of diagnosis, veterans reporting having money to cover basic needs were 
significantly less likely to have post-deployment adjustment problems." Veterans with mental health and substance abuse disorders often experience the greatest employment challenges emphasizing the importance of focusing efforts for sustaining employment versus attaining employment (IOM, 2013).

\section{Veteran Transitions into Postsecondary Education}

Many Army veterans enroll in postsecondary education with use of the Post 9/11

GI Bill incentive (Ackerman, DiRamio, \& Garza-Mitchell, 2009; Church, 2009; Clemens \& Milsom, 2008; Zinger \& Cohen, 2010). Ryan et al. (2011) suggests enrollment of veteran students from OIF/OEF is highest at 2-year public colleges, though many veteran students report being underprepared for academia (Ackerman et al., 2009). Veterans enrolled in four year institutions often fail to graduate or take longer to graduate as attrition rates range between 46-88\% (Cate, 2014; Wood, 2013). Feelings of poor preparation stem from a perceived loss of time spent in the military (Ackerman et al., 2009), while also reporting relational or social interaction challenges with typical students (DiRamio \& Spires, 2009; Johnson, 2009; Olsen, Badger, \& McCuddy, 2014). Limited investigation has been performed for student veterans of OEF/OIF (Ackerman et al., 2009; DiRamio, Ackerman, \& Mitchell, 2008; DiRamio \& Spires, 2009; Ford, Northrup, \& Wiley, 2009; Johnson, 2009; Rumann \& Hamrick, 2009) with only two available studies performed from an occupational therapy perspective (Plach \& Sells, 2013; Tomar \& Stoffel; 2014). These studies all identify specific challenges associated with transitioning in a novel sociocultural environment, thus evoking changes to veterans' identities, daily habits, roles, and routines. 
DiRamio, Ackerman, and Mitchell (2008) developed the first grounded theory study to explain the challenges student veterans experience as they transition into postsecondary education. DiRamio et al. applied the "Moving In, Moving Through, Moving Out" model for adult transition by Schlossberg, Lynch, and Chickering (1989) to explain how student veterans cope with transitions into the military, through deployments, out of the military and entering education. Similar to other student veteran studies, their results suggested issues of "blending in, connecting with peers, establishing a new identity, and obtaining financial and disability benefits" (Ackerman et al., 2009; DiRamio et al, 2008; Jones, 2013; Olsen et al., 2014; Tomar \& Stoffel, 2014).

An additional finding identified by DiRamio et al. included negative commentary by faculty and peers perceived as "anti-military" by student veterans. The researchers suggested these experiences served as barriers to connecting with the university and compelled veterans to suppress their military identity. This finding suggests the need to assess campuses for cultural awareness and sensitivity towards student veterans as faculty attitudes and behaviors can impact students' decisions in leaving college (Lundquist, Spalding, \& Landrum, 2002). Though little is known about faculty members' perceptions of student veterans (Banard-Brak, Bagby, Jones \& Sulak, 2011), other studies have been performed to identify institutional perceived supports available for wounded warriors (Vance \& Miller, 2009).

Vance and Miller (2009) performed a cross-sectional study to identify the role of disability service offices in supporting wounded warriors at educational institutions across the U.S. and found that psychological, mobility, and learning disabilities comprised $90 \%$ of the disabilities veterans experienced in the sample surveyed. Elliott, 
Gonzalez, and Larsen (2011) screened for psychological problems in their predictive study of 124 veterans transitioning to postsecondary education. Their study provided credence to Vance and Miller's as more exposure to combat demonstrated an increase in PTSD symptoms and the perceived emotion of alienation by veterans on campus. Psychological problems associated combat service were further confirmed in a national sample of over 600 student veterans surveyed with $24 \%$ experiencing severe depression, $35 \%$ severe anxiety, and 46\% endorsing PTSD symptoms (Rudd, Goulding, \& Bryan, 2011).

Ackerman, DiRamio, and Garza-Mitchell (2009) performed the seminal qualitative narrative study in student veterans transitioning to postsecondary education. Twenty-five student veterans transitioning back into college life following deployments to OIF/OEF were interviewed and acknowledged key challenges to the transition. Veterans reported working through the VA bureaucracy was not easy as it prolonged receipt of educational and medical benefits. Students resorted to out-of-pocket expenses for tuition and fees until educational benefits were received. This became an unexpected financial burden that other authors have echoed in the provision of financial assistance via institutional loan payment processes and/or individualized money management education for veterans (Snead \& Baridon, 2010).

Veterans also reported readjustment difficulties that included (1) needing to relearn study skills due to a prolonged absence from academia, (2) coping with the psychosocial effects of war (e.g., PTSD, anxiety or depressive symptoms), and (3) transitioning from the structured military life to a loosely configured campus environment; all serving as barriers for veterans" "fitting in" on campus (Ackerman, et 
al., 2009). Other studies have confirmed this problem as a perceived inability to relate and/or build new relationships with traditional students who are younger and less mature (DiRamio et al., 2008; Rumann \& Hamrick, 2010; Tomar \& Stoffel, 2014).

The limitation in veteran transition studies into the postsecondary education context using an occupation-based perspective has been described within the introduction of this dissertation. As a reminder, only two investigations exists (Plach \& Sells, 2013; Tomar \& Stoffel; 2014) which establishes an opportunity for occupational scientists and occupational therapy researchers to develop further inquiries into this under-researched area. Plach and Sells (2013) identified that the occupation of education was an area of prioritized concern within $70 \%$ of their sample studied. In fact, school was the second highest priority area with concerns reported in "relating to younger classmates" and "having the essential skills for the academic environment" (Plach \& Sells, 2013).

Tommar and Stoffel's (2014) examination of the lived experiences of two student veterans incorporated the novel use of the Photovoice methodology (Wang \& Burris, 1997). Tommar and Stoffel were able to collect meaningful images from veterans that were analyzed for themes across both participants' data in this seminal qualitative occupation-based study examining student veteran transitions. Four emergent themes were identified: (1) reminiscence of past duty, (2) transition from military to civilian student life, (3) entry to a new stage in life, and (4) influences of the university and community context (Tomar \& Stoffel, 2014). Given the small sample size of the study, all four themes appeared to align with previous studies into identity development (Jones, 2013; Olsen et al., 2014), relational challenges (Ackerman et al., 2009; DiRamio et al, 2008), and institutional administrative issues (Ackerman et al., 2009; DiRamio, 
Ackerman, \& Mitchell, 2008; DiRamio \& Spires, 2009; Ford, Northrup, \& Wiley, 2009; Griffin \& Gilbert, 2015; Johnson, 2009; Rumann \& Hamrick, 2009). This study acknowledged the small sample size as a major limitation, which places emphasis on the need for another investigation into the lived experiences of student veterans to be performed from an occupation-based perspective.

These transition issues identified within the postsecondary education context suggests a difference experienced between veterans and typical traditional age students that comprise the majority of student culture. The occupational therapy literature, specifically, is lacking a firm foundation for guiding occupational therapy practice and research. A theoretical orientation for conceptualizing the experiences of student veterans is clearly needed for occupational therapy practice, especially from a culturally relevant or sensitive approach given the uniqueness of veteran experiences compared to their typical student peers. The need for a culturally relevant theoretical conceptualization guided the research of this dissertation.

\section{Transition Opportunities for Occupational Therapy}

Serving on combat deployments has numerous emotional consequences that can manifest in pathological problems that disrupt occupational engagement (Plach \& Sells, 2013; Radomski \& Brininger, 2014; Tomar \& Stoffel, 2014). Examination of the transition experiences of veterans can illuminate underlying strengths needed to facilitate health and wellbeing. The cathartic deployment experiences of combat veterans also develop assets that have been considered transformational and even positive (Tedeschi, 2011). For instance, experiencing combat has demonstrated positive outcomes for increasing emotional maturity needed to be more disciplined (Aldwin \& Levenson, 2005; 
Aldwin, Levenson \& Spiro, 1994; Casella \& Motta, 1990; Elder \& Cripp, 1988).

Tedeschi (2011) describes these experiences of resilience in the face of mortality and morbidity as "post-traumatic growth." Understanding veterans' growth experiences proves critical in rapport building as veterans aspire for validation of their individualized contributions, thus illuminating areas of strength needed for promoting positive health and wellbeing.

Cogan (2014) astutely acknowledges that literature for veterans of this war era focuses primarily on mental health problems. This presents a particular challenge for occupational therapists as many are not familiar with mental health practice nor provide direct service into this practice area (Gibson, D'Amico, Jaffe, \& Arbesman, 2011). Occupational therapists, however, are uniquely trained in treating veterans holistically through evaluation of positive emotional states, psychological and social functioning, productive activities, and resilience (AOTA, 2010). As previously stated, mental health problems are prevalent in veteran populations where complexities lie in teasing out deficits in cognition, emotion, behavior, and social functioning (Clark et al., 2007, 2009). Therefore, it is imperative that occupational therapists working with veterans familiarize themselves with evaluation and treatment strategies required to support recovery processes in the cognitive, emotional, social, and behavioral dimensions.

Occupation-based evidence exists for reducing anxiety and enhancing quality-oflife for service members experiencing the acute stressors of combat (Stoller, Greuel, Cimini, Fowler, \& Koomar, 2012). One recent feasibility study demonstrated a reduction in PTSD and depressive symptoms for a cohort of veterans interested in a high-intensity sports program provided in the VA (Rogers, Mallinson, \& Peppers, 2014). Another 
recent study using hatha yoga as a behavioral health intervention reduced anxiety, depression, and PTSD symptoms (Speicher, Walter, \& Chard, 2014). Other studies have employed creative methodologies to investigate the effects of TBI or life-skill focused programs for mental health and driving specific outcomes (Beck, Gonzales, Sells, Jones, Reer, \& Zhu, 2012; Classen, Monahan, Canonizado, \& Winter, 2014). The limited availability in occupation-based empirical studies suggests the need for more high quality descriptive research aimed at building a firm foundation for empirical study in veteran transitions (Pierce, 2014, p. 8; Tomar \& Stoffel, 2014). Numerous review papers exist to guide occupational therapy treatment and research endeavors, thus highlighting specific skill development areas performed by military occupational therapists needed for productive work, self-care, employment, and education (Ellsworth, Sinnott, Laedtke, McPhee, 1993; Gerardi, 1999; Rice \& Gerardi, 1999; Mattila, Crandall, \& Goldman, 2011; Montz, Gonzales, Bash, \& Carney, 2008; Smith-Forbes, Najera, \& Hawkins, 2014) with the most extensive review authored by Erickson, Brininger, Newton, Mattila, and Burns (2011) for occupation-based practice. For recommendations on traumatic brain injury evaluation and treatment of veterans with TBI, Radomski, Davidson, Voydetich and Erickson (2009) recommends occupational therapists focus on vision, self-awareness, cognition, life roles, and daily tasks to enhance occupational performance.

\section{Implications for Occupational Therapy Practice}

The multifaceted issues veterans experience in occupational dysfunction, employment, and education fall within the scope of the American Occupational Therapy Association's (2007) Centennial Vision as veterans require support in overcoming 
societal obstacles in order to participate in valued activities. The following implications are presented to support veterans in transition:

- The need for programs and research that support rich descriptions of veteran narratives or oral histories, affirming the extensive contributions sacrificed for national interests as this assists in the identity renegotiation of transitioning service members.

- The need for occupational-based qualitative and quantitative research devoted to the transition needs and outcomes for veterans; primarily emphasizing education and employment which comprise under researched areas.

- Occupational therapy scholars should assess student veterans' perspectives on resource utilization, support, and/or outreach efforts provided by universities.

- Occupational therapists evaluating veteran work performance should assess mental health outcomes and devise programs that sustain employability.

- Outcome research is needed in the areas of recovery, resilience, and community reintegration for veterans in transition (Radomski \& Brininger, 2014). Specifically, mTBI outcomes need to be evaluated for productive work, education, employment, and driving.

\section{Conclusion}

Therapists and educators alike need to understand the complex challenges veterans face during their transition to civilian life. Regardless of each veteran's health status, adaptation to the civilian environment requires navigation around life circumstances, thus posing an important opportunity for occupational therapists to guide Gulf War II era veterans in transition. Utilizing the findings provided in this review may 
assist occupational therapists in understanding how occupation can support the needs of military service members in transition to the civilian context. More importantly, the review also illustrates the gaps in which further research on occupation need to be completed that may directly support veterans in transition.

Copyright $\odot$ Brian Tuan Gregg 2016 


\section{Chapter Three: Well-being and Coping of Student Veterans Readjusting Into}

\section{Academia: A Pilot Survey}

\section{Introduction}

More than 2.2 million military veterans are at risk of disruptions of engagement in daily occupations following a combat tour to Iraq or Afghanistan (Plach \& Sells, 2013). Following discharge from active duty service, an increasing number of veterans begin their transition from the military with the intentions to enroll in postsecondary education (Ackerman et al., 2009) thereby using the post 9/11 GI Bill entitlement. An American Council on Education [ACE] report by McBain, Kim, Cook, and Snead (2012) indicates that over 500,000 military veterans have enrolled in postsecondary education since 2009 and rates are expected to increase with the systematic down-sizing of the military. But, with 39 billion dollars invested in the Post 9/11 GI Bill since 2008 and low rates of graduation (Cate, 2014), universities must identify supports for these non-traditional students with unique life experiences.

As the population of student veterans continue to grow (Ellison, Mueller, \& Smelson, 2012), universities must be aware of the unique challenges they experience. Veterans returning to school after combat are often characterized as non-traditional students, defined as an adult learner returning to academia following unique life experiences and contexts (McBain et al., 2012). They may formulate habits and cognitive strategies considered atypical to traditional student development, and face additional challenges when entering academia. In addition, student veterans may struggle with the losses of personal growth, individual aspirations, and interpersonal relationships as they transition into academia (Holloway, 2009). Veterans moving toward 
reintegration after deployment may require assistance to refocus their energies, cope with a change in work environments, and family or friendship separations as they reconstruct meaning in their lives (Holloway, 2009). Promoting awareness and support are required to help assist in the readjustment of military veterans and to advance their aspirations in higher education.

Recent research describes the process through which military veterans transition and reintegrate within their local community following an honorable discharge (BarnardBrak, Bagby, Jones, \& Sulak, 2011). Further studies have discussed the potential challenges veterans face when transitioning from the military (Bellotti, Laffayea, \& Weingardt, 2000). The body of literature describing student veterans' transitions to postsecondary education focuses on generalized social experiences, (Ackerman et al., 2009; Jones, 2013; Olsen et al., 2014; Rumann \& Hamrick, 2010; Tommar \& Stoffel, 2014) pathological screening for psychological disorders, (Elliott et al., 2011; Plach, \& Sells, 2013; Rudd et al., 2011) and role identification of institutional supports (Daly \& Garrity, 2013; Vance, \& Miller, 2009). However, the literature is limited in the number of studies that describe the experiences of student veterans readjusting to civilian life within the postsecondary education context and the factors used to cope with the transition (DiRamiro et al., 2008; Griffin \& Gilbert, 2015).

The current study adds to the existing literature on the student veteran transition process by incorporating the conceptual transition framework outlined by Anderson, Goodman, and Schlossberg (2011) to collect individual responses on perceived assets and liabilities for coping with the transition to postsecondary education following combat military service. Due to the paucity of literature describing coping strategies for student 
veterans in transition to postsecondary education, the authors decided to investigate student veterans' perceived assets and liabilities. This study provides a quantitative measure of perceived attitudes and beliefs in regards to coping with the transition from the military to postsecondary education. Therefore, the purpose of this pilot survey study was to describe the attitudes and beliefs of student veterans coping with an adult transition to postsecondary education. This pilot study had two aims: 1) to identify intrinsic and extrinsic factors perceived as barriers and/or supports for transitioning to postsecondary education, and 2) to identify the critical needs of student veterans consistent with the domain of the occupational therapy practice.

\section{Methods}

\section{Adult Transition Framework}

Student veterans undergo a significant life change when transitioning from the military to postsecondary education that can be examined from a holistic lens. Anderson et al. (2011) posit that adults in transition are, "often confused and in need of assistance" (p. 37). Anderson et al. incorporate Schlossberg, Lynch, and Chickering's (1989) model of Adult Transitioning into their conceptual framework to guide counselors in adult transitions that view the individual holistically. Schlossberg, Lynch, and Chickering's model is instrumental in framing questions regarding the transition experiences of student veterans (DiRamio et al., 2008; Griffin \& Gilbert, 2015) as it illustrates how adults "move in, through, and out" of multiple life transitions (Anderson et al., 2011). These transitions have varying effects on adult lives depending on how each individual appraises the transition event being approached. Furthermore, the model provides a unifying structure to contextualize the transition and offers potential resources or factors 
for depicting and changing responses over time in the form of the "4 S System" (pp. 6193). The 4 S's represent situation, self, support, and strategies and may be considered an asset or liability for the individual in transition. The $4 \mathrm{~S}$ system provided the theoretical and guiding framework for each survey item developed within this pilot study, crosssectional survey design. Each item developed corresponded with one of the $4 \mathrm{~S}$ factor variables.

\section{Instrument Development}

A 40-item Qualtrics ${ }^{\mathrm{TM}}$ questionnaire composed of 28-Likert scale questions and 12 demographic/open-ended questions was developed on attitudes and perceived behaviors of respondents experiencing the transition to postsecondary education. The preliminary questions reflected each of the $4 \mathrm{~S}$ System factors outlined by Anderson et al. (2011). A two part process was performed to attain content validity of the questionnaire. First, the questionnaire was submitted to a group of IRB approved military clinical researchers working directly with service members discharging from active duty for expert review. The researchers were each sent a memorandum outlining the purpose and objectives of the study, the preliminary questionnaire, and an agreement rating form using a scale of 1-4 to rate each item. The items were rated on the level of representativeness, the clarity of the question, and to which one of the " $4 \mathrm{~S}$ 's" transition factors (i.e. self, situation, support, and strategies) did the item most represent.

Secondly, the questionnaire was revised following expert review and then sent to a group of student veterans to further evaluate the content validity of the questionnaire from a respondent perspective using the previous procedures applied by expert reviewers. The level of agreement was calculated using the Content Validity Index (CVI) described 
by Lynn (1986) for item and overall scale agreement. Once the questionnaire achieved the cutoffs for overall CVI scale, it was made available for respondents and disseminated online.

\section{Study Design}

The "Tailored Design" method described by Dillman, Smyth, and Christian (2009) was used to guide the development of this pilot survey study for veteran respondents at a large research university. The Tailored Design method is a scientific research design that, "reduces four principles of survey error: coverage, sampling, nonresponse, and measurement error" (Dillman et al, 2009). This design implemented a set of procedures for the sample to follow and encouraged all respondents to complete the survey as a positive social exchange. The respondents provided informed consent and voluntarily chose to participate in the administered surveys. The surveys were administered online via a LISTSERV from the local university Veteran Resource Center or (VRC). All questions and concerns were addressed by the principal investigator. The authors used the Qualtrics ${ }^{\mathrm{TM}}$ software program for the development of the survey instrument.

\section{Subject Recruitment}

Respondents were recruited for inclusion in the study primarily from the University VRC. The VRC provided a centralized location in which eligible participants congregated and communicated with other student veterans. Recruitment posters were printed with an assigned Quick Response (QR) barcode and posted in communal areas and disseminated by-hand at the university. The principal investigator (PI) also posted recruitment flyers at key locations on campus that student veterans reported routine 
interaction with peers. The VRC Director also maintained a university LISTSERV for student veterans which allowed the researcher to send the recruitment poster and survey link to the eligible population. Follow up electronic mail notifications were sent on a monthly basis. Eligible participants were approached with information as approved by the VRC director. The PI provided the standard description of objectives for the survey to eligible participants and answered any questions that the respondents had.

Respondents were free to decline survey completion and/or omit answers to survey questions. Also, to reduce coercion and study bias, the VRC Director was blinded to respondents of the anonymous survey. Figure 3.1 illustrates the recruitment strategy.

Figure 3.1 Flow chart for respondents included in transition survey results.

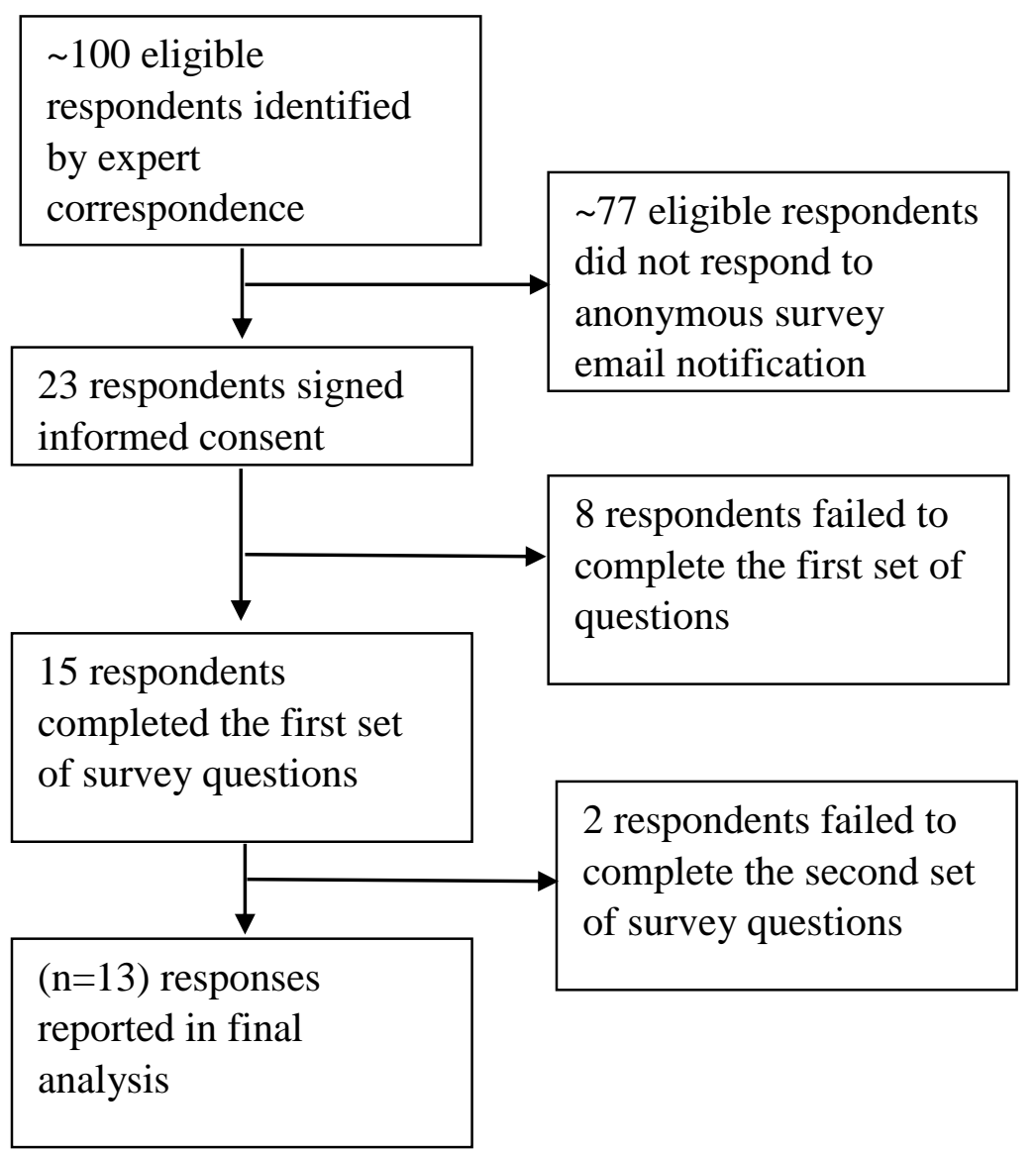




\section{Study Sample}

Student veterans actively enrolled at the University of Kentucky served as the study population. Respondents met inclusion if: they were between the ages of 20-45, were no more than 3 years removed from a combat tour, and able to speak, write and understand the English language. The researchers established cut-offs for combat military service (within the past 3 years) to limit recall bias for previous military experiences affecting the current transition. Any participant meeting inclusion criteria was considered for enrollment in the study, regardless of gender or ethnic background. Respondents were excluded if they reported current active service to a United States military service branch. The authors excluded respondents with current active duty service in order to maintain homogeneity between respondent transition experiences. Also, this study focused on coping with transition from a perspective of change in personal roles, habits, and routines affected by acculturation into postsecondary education which would likely vary from an individual that maintains their military identity and/or status. Table 3.1 summarizes the respondent demographics. 
TABLE 3.1 Demographics of Respondents

\begin{tabular}{|c|c|c|}
\hline Variable of Interest & \multirow[t]{2}{*}{$\mathrm{N}$} & \multirow[t]{2}{*}{$\%$} \\
\hline Gender & & \\
\hline Male & 11 & 85 \\
\hline Female & 2 & 15 \\
\hline \multicolumn{3}{|l|}{ Age } \\
\hline $18-20$ years & 0 & 0 \\
\hline 21-24 years & 2 & 16 \\
\hline $25-30$ years & 7 & 58 \\
\hline $31-39$ years & 3 & 25 \\
\hline $40+$ years & 0 & 0 \\
\hline \multicolumn{3}{|c|}{ Current Post-secondary Educational Level } \\
\hline Freshman & 1 & 8 \\
\hline Sophomore & 5 & 38 \\
\hline Junior & 3 & 23 \\
\hline Senior & 3 & 23 \\
\hline Graduate Student & 1 & 8 \\
\hline \multicolumn{3}{|l|}{ Marital Status } \\
\hline Single & 8 & 62 \\
\hline Married & 4 & 30 \\
\hline Separated & 0 & 0 \\
\hline Divorced & 1 & 8 \\
\hline \multicolumn{3}{|l|}{ Previous "Time in Military Service" } \\
\hline $1-3$ years & 0 & 0 \\
\hline $3-5$ years & 7 & 54 \\
\hline $5-10$ years & 5 & 38 \\
\hline $10+$ years & 1 & 8 \\
\hline \multicolumn{3}{|c|}{ Number of Deployments Experienced } \\
\hline 0 & 0 & 0 \\
\hline 1 & 4 & 30 \\
\hline 2 & 5 & 40 \\
\hline 3 or more & 4 & 30 \\
\hline \multicolumn{3}{|c|}{ Currently Receiving VA Medical Care } \\
\hline Yes & 6 & 46 \\
\hline No & 7 & 54 \\
\hline \multicolumn{3}{|c|}{ Perceived Category of VA Medical Care } \\
\hline Physical & 2 & 29 \\
\hline Mental & 1 & 13 \\
\hline Both & 2 & 29 \\
\hline None of the above & 2 & 29 \\
\hline
\end{tabular}

Note. $(N=13)$ 
This study reports a comparative sample to the active military population (Military OneSource, 2012) with 85\% male and 15\% female respondents. The majority of respondents were between the ages of 25-30 years at 58\% and $92 \%$ were undergraduates. Married student veterans comprised 30\% of the sample and $8 \%$ reported being divorced. This current sample reported $54 \%$ as serving 3-5 years in military service. This percentage denotes that the respondents had either enrolled in postsecondary education after completion of their initial active duty service obligation or separated early due to an honorable discharge that was medically related. An interesting finding for this small sample size was the high report of multiple deployments at $70 \%$. This finding would reflect the commonality of systematic continuous deployments currently experienced by military culture. Also, $46 \%$ reported receiving Veterans Administration (VA) assistance for healthcare which would denote perceived service connection for an underlying medical disability incurred during military service. Respondents reported receiving VA services for a perceived physical disorder at $29 \%$ and $42 \%$ reported care for a perceived mental health related disorder.

\section{Results}

Table 3.2 summarizes the results of the pilot study. The final data was aggregated and reported as frequency data relating to percentage of agreement and frequency of two or more episodes per week. We report a total of 13 completed surveys to equal a $13 \%$ response rate with 11 male respondents and 2 female respondents. Twenty-three respondents initially consented to participate in the study with 10 failing to complete all of the survey items required for the final analysis. Each item response reported in the 
TABLE 3.2 Coping Mechanisms Reported by Student Veterans Experiencing an Adult Transition: Percentages Survey Questions Adult Transitioning Factor

Use of military decision-making skills in school.*

No negative mental effects following combat service.*

Uses military experiences/training to manage stressors.*

Manages memories of combat service in a healthy manner.*

Never in imminent danger during deployment.*

Did not engage in enemy contact.*

Had a detailed plan after the military.*

Manages finances without difficulty.*

Reports healthy outlet for managing stressors.*

Considers Transition normal compared to peers.*

Veterans' needs are supported at their university.*

Confident in completing academic requirements on time.*

Uses exercise to manage stress.*

Fulfills daily roles within family.+

Engages in activities that produce an identity different from Soldiering.+

Achieves restful sleep.+

Eats healthy meals.+

Bounces back from adversity.+

Reports energy to accomplish daily tasks.+

Experiences financial issues.+

Communicates with peers or family members.+

Sustains concentration on academic studies.+

Moments of feeling down or in the dumps.+

Experiences stressful combat-related memories.+

Distressed with academic requirements.+

Seeks out people or situations for support within the university.+

Engages in a routine of physical exercise.+

Utilizes the campus Veteran Resource Center.+

\begin{tabular}{ccc} 
Strategies & 10 & 77 \\
Support & 4 & 31 \\
Strategies & 12 & 93 \\
Strategies & 11 & 85 \\
Self & 2 & 15 \\
Support & 8 & 62 \\
Support & 9 & 69 \\
Self & 8 & 62 \\
Strategies & 10 & 77 \\
Strategies & 7 & 54 \\
Support & 8 & 62 \\
Support & 9 & 69 \\
Support & 11 & 85 \\
Support & 6 & 46 \\
Support & 9 & 69 \\
Support & 5 & 38 \\
Strategies & 11 & 85 \\
Strategies & 11 & 85 \\
Support & 11 & 85 \\
Support & 3 & 23 \\
Support & 8 & 62 \\
Support & 10 & 77 \\
Situation & 2 & 15 \\
Support & 1 & 8 \\
Self & 2 & 15 \\
Support & 5 & 38 \\
Strategies & 9 & 69 \\
Support & 5 & 38 \\
\hline & &
\end{tabular}

*Reported in Percentage of Agreement

+ Frequnecy reported more than 2 times a week 
final analysis corresponds with one of the following transition factors attributed to the $4 \mathrm{~S}$ System.

\section{Factor Items Representing Situation}

The transition factor of "Situation" represented an event or nonevent appraised by the individual as having an impact or change in their current life situation and varied by "what was happening" and if there were "multiple stressors" at the time of the transition (Anderson et al., 2011, pp. 61-72). In the present study, only one item was developed to represent negatively appraised events occurring within the student veterans' transition to postsecondary education. A report of "feeling down in the dumps" during the transition satisfied multiple dimensions outlined by Anderson et al. in an appraisal of their transition situation such as timing, duration, concurrent stress, and/or a self-assessment of the negatively appraised event (pp. 72-73). Feeling, "down in the dumps" greater than twice a week could suggest a current depressive episode or even previous history of depression exacerbated in the current transition. This was a significant variable of interest to include considering the substantial mental health problems associated with Post 9/11 era veterans (Hoge et al., 2004; Hoge, Auchterlonie, \& Milliken, 2006; Hoge, Terhakopian, Castro, Messer, \& Engel, 2007; IOM, 2013; Smith et al., 2008; Tanielian \& Jaycox, 2008). Therefore, the situation factor item focused on identifying the percentage of depressed feelings associated with the transition to postsecondary education. Encouragingly, a limited number of respondents reported moments (twice a week or more) of feeling down or in the dumps at $15 \%$.

\section{Factor Items Representing Self}

The factor of "Self" was represented in responses that alluded to life altering events and how each individual used their innate "assets and liabilities" to navigate the 
challenges of transitioning to postsecondary education. Each individual brought a unique set of assets and liabilities that influenced how they coped with the transition (Anderson et al., 2011, p. 83). One insight into the previous life experiences of this sample consisted of involvement in life threatening situations during deployment. For instance, $85 \%$ reported experiencing imminent danger during their deployed experiences, though deployment experience seemed to be expressed as an asset in this sample. This finding reflected exposure to a war-time context in which the potential to be harmed was real, though strengthened their capacity to overcome obstacles by promoting resilience over life circumstances.

Important findings were also identified in student veterans' perceptions of financial management and reported stress levels with current academic requirements. Sixty-two percent of student veterans reported the ability to manage finances without difficulty. Financial management challenges appear to be a common theme in student veteran studies (Ackerman et al., 2009; DiRamio et al., 2008; DiRamio \& Spires, 2009; Rumann \& Hamrick, 2009). However, one positive finding alluded to each respondent's innate coping ability to current academic requirements as low levels of perceived stress were reported in $15 \%$ of the respondents for current academic stress.

\section{Factor Items Representing Support}

The factor of "support" was used for items that represented experiences requiring assistance from university services such as academic and administrative support, veteran resource support, and university counseling services. In response to a question regarding the negative mental effects following combat service, $31 \%$ of respondents reported experiencing negative mental effects defined as difficulty with memory, concentration, 
and/or mental health service seeking, though only $8 \%$ reported experiencing stressful combat-related memories. Many of the respondents reported limited experience with engaging enemies in combat as $62 \%$ had not engaged in direct enemy contact, defined as firing upon an enemy target. The majority of student veterans (69\%) identified a preliminary plan for their transition following their military service. Identity renegotiation was a limited concern for this sample as $69 \%$ reported engagement in activities that produced an identity different from soldiering.

With regard to perceptions of the current university programs, $62 \%$ reported the attending institution as one that supports veterans' needs. A large proportion of respondents reported moderately high self-efficacy in their abilities to attain a degree, as $69 \%$ were confident that they could complete their academic studies on time. Sixty-nine percent reported the ability to maintain concentration on their studies. Interestingly, only $38 \%$ sought out people or situations for support within the university and similarly used the local campus veteran resource center.

In regards to supporting psychosocial well-being, this sample's responses were mixed towards optimizing methods of promoting well-being as $85 \%$ utilize exercise to manage their stress but, perceptions of role fulfillment were low as only $46 \%$ reportedly fulfill daily roles within their family. Furthermore, only $38 \%$ reportedly achieve restful sleep yet, $85 \%$ report having enough energy to accomplish daily tasks. A low percentage reportedly experience financial problems (23\%) at the time of survey completion. Also, $62 \%$ maintain a form of communication with their family members or peers. 


\section{Factor Items Representing Strategies}

Coping factors based on individualized "strategies" were identified in items of this online questionnaire for managing the stressors associated with transitioning to postsecondary education. Seventy-seven percent of respondents reportedly used previous military decision-making skills for school. A large majority (93\%) of respondents reported a high use of military training and experiences to manage stressors, which provides insights into where occupational therapists may focus on strategy development. Intrusive symptoms reflective of post-traumatic stress appeared to be low in this sample as $85 \%$ of respondents reported the ability to manage memories of combat service in a healthy manner. In fact, the majority (77\%) of respondents also acknowledged access to a healthy outlet for managing their stressors. Of note, $54 \%$ of student veterans considered their transition normal when compared to their peers defined as other students. Eighty-five percent reported healthy eating habits and the ability to bounce back from adversity. Lastly, 69\% reportedly maintained a routine of physical exercise following their military discharge. The findings attributed to the coping strategies factor all highlight the active use of healthy coping strategies applied by the current sample of student veterans.

\section{Open-Ended Responses}

The online questionnaire provided open-ended text boxes for student veterans to elaborate on any response. These text boxes were used to ask student veterans to describe any perceived challenges that were not covered by the Likert based items as well as any typical stress management response used. Results of the textual responses were grouped according to the emergent themes of the responses. The following themes 
emerged from open-ended responses: relational challenges, cognitive challenges, financial challenges, no perceived concerns, and stress management.

Relational Challenges. Many open-ended responses described difficulties in socially relating to college students without military experience. Student veterans endorsed engaging in conversations and interacting with peers but, found the "age gap" or "relating to peers of a different age" as a problem area within their social environment. Many reported difficulty, "being nice to others" which could represent a difference in acculturating to a less structured or serious environment such as the military. This sentiment was reflected in the following statement:

Dealing with younger students is difficult because they have no real perspective on life and don't understand what real life is like. No amount of personal discipline.

Student veterans did not seem to express difficulty relating to the course material or academic assignments required of all students, rather they reminisced over missing the "comradery" experienced in the military where relational bonds were easier to develop from shared experiences, duties, and responsibilities. Being in an educational setting offered a new environment in which relational bonds were more difficult to develop as shared experiences simply did not exist and required effort to construct. The social norms of education resembled a culture foreign to that experienced within the military thus, lacking shared experiences to derive meaning and relational assumptions. The following comment embodied this notion: 
Associating with non-veterans is a challenge. They don't understand everything we've been through, and it's simply aggravating at times to be thanked for my service constantly.

Cognitive Challenges. Student veterans reported difficulties with routine cognitive tasks such as sustaining attention, prioritizing tasks, managing time, and memorizing new learning material. The difficulties were attributed to "the fast pace at the university" or "switching mental thought processes" when focusing on a variety of class related assignments lacking a clear order or hierarchy to complete tasks in a manner that student veterans are accustomed. Some student veterans attributed "the lack of structure" as the reason "prioritizing tasks are difficult." Aptitude was not considered a concern for prioritizing tasks, rather, concerns expressed included the absence of external structure, such as clear and concise guidance explaining what tasks need to be done, how they need to be done, and when. For some student veterans, these feelings were compounded by life circumstances associated with being a non-traditional student. One student veteran described their non-traditional student circumstances and negative feelings experienced in the passage below:

I have a difficulty making sufficient time for school and my husband and son, so I often feel like I'm failing at both.

This was a common challenge faced by student veterans because they find that they have to re-learn how to prioritize tasks, manage time, and memorize new materials in short durations of time with little insight as to how to function in a non-military setting.

Financial Challenges. A few student veterans described aspects of maintaining a military career that were missed and often underestimated such as no longer receiving a 
wage or "consistent paychecks" after leaving the military. Other veterans reported issues incurred during their military service that affected current job prospects and were difficult to overcome as a student. In particular, this student veteran described how indebtedness affected career prospects that required a high level security clearance:

I think if you have bad credit because you thought you could work while going to class, then your credit shouldn't make you unemployable.

The student veteran further elaborated their concerns in this statement:

The most difficult challenge for me was trying to correct the financial liabilities I had gotten myself into since I was expecting to be able to work while going to school. This was not possible to do both. Since starting at [school] I have been homeless, had my car repossessed and other difficulties. As it stands I cannot improve this situation because the job I am qualified and trained to work in will not hire me because of my now destroyed credit history apparently makes me a security risk. Because I, a combat veteran and Marine would ever do anything that would bring dishonor to my country. So the thing I wish I had done differently was not to have bought a car or other things and I wish my job would have been flexible with my hours.

This example demonstrates the unique challenges student veterans experience as their military skills, training, and experiences are limited to narrow career fields easily compromised by financial misgivings.

No Perceived Concerns. A few student veterans reported positive transition experiences in postsecondary education. Many of the positive experiences reflected how each individual appraised the academic environment. For one veteran, the transition was 
a part of their autonomous decision making that fit within their goals set prior to leaving the military:

Coming back to school was what kept me aligned when I was in the service.

The transition has been smooth because I wanted it to be.

Another student veteran provided a comparison of their current experiences with the hardships endured during combat. They identified the transition as a minor event compared to the adverse life experiences combat entails:

I am an actual combat Vet. I was in the Infantry. I've done two deployments. The vast majority of the combat I saw was in Afghanistan. My unit was stationed in Panjwai District, Kandahar Province. It was one of the hottest areas in Afghanistan and we performed daily patrols in kinetic operations with the intent of combating the Taliban. In terms of my civilian life, my academic record, successful financial situation, and long term career and educational goals are evident of my well-being as a veteran who has transitioned.

Stress Management. Many student veterans endorsed a pattern of healthy behaviors used to manage stressful situations experienced during their transition. For instance some pattern of "exercise" or routine spiritual practice such as "yoga" and "meditation" was implemented to deter stressful responses. Others engaged in social interaction in the form of "social gatherings with friends" or "family" or even focused on "self-reflection." One veteran acknowledged the role family played in managing their stress and providing a relief from their circumstances:

I rely on my team/family to be strong when I cannot, I try to get some mental distance so I can put the problem in a realistic perspective. 
Some veterans reported the use of negative coping behaviors that place the individual at risk to adverse health consequences. For instance a few veterans reported the use of illicit "recreational drugs" and consistent "smoking" and "drinking" or consumption of alcoholic beverages. These findings are not surprising when considering the prevalence of substance use reported in military populations (Hoge, Auchterlonie, \& Milliken, 2006).

\section{Discussion}

This study contributes to the modest body of literature describing students veterans' experiences when transitioning to postsecondary education. Previous studies have explored veterans' experiences from a qualitative approach using Anderson, Goodman, and Schlossberg's model of transitioning and recommend the model as the preeminent guiding theoretical approach (DiRamiro et al., 2008; Griffin \& Gilbert, 2015). The novel contribution of this pilot study was the use of the transition framework described by Anderson et al. that guided survey development on reported coping responses student veterans experience for perceived transition stress. Though the results of coping responses are limited to this small sample of student veterans, many responses confirmed previously reported findings and provided unique insights into newly discovered areas.

Insights were provided into how student veterans appraise transitioning and to what degree they used the coping methods outlined in this study. The predominant findings resonated within the factors of support and strategies. More than a third of respondents (38\%) experienced difficulty managing their finances indicating the need for external or environmental support. This is commonly identified in the literature for 
student veterans, (DiRamio et al., 2008; Gonzalez \& Larsen, 2011; Griffin \& Gilbert, 2015; McBain et al., 2012; Rumann \& Hamrick, 2010) as financial problems experienced often result from practical situations such as unresolved credit debts for leases running out and unexpected economic costs incurred while awaiting housing allowances, tuition payments, and book stipends to begin from the VA. Student veterans may underestimate the financial burden associated with the transition and lack awareness on the amount of entitlement they anticipate receiving from VA and for the length of time it may take to receive monetary benefits; they may also underestimate if they will be able to support themselves or family members with the monetary benefits they receive.

Service members unaware of impending military separations such those affected by injury, impairment, or even Reduction-In-Forces (RIF) military separation boards may transition from the military in haste, lending to miscalculations in preparations for standard-of-living changes. Student veterans grow accustomed to housing allowances or governmental subsidized living when serving on active duty, which all goes away as they transition to civilian living. The military offers transition assistance planning and education resources, however, veterans prefer to solve their own problems with their transition experience as evidenced in this study. This would suggest that the majority of veteran respondents endorsed coping with the transition process in a "problem-focused" pattern of coping versus "emotion-based" as it pertained to specific practical events (Lazarus \& Folkman, 1984). This is considered a positive finding as it suggests student veterans are more focused on working through the sources of their transition stressors, (Schwarzer, Starke, \& Buchwald, 2003) however, further research is warranted to 
correlate definitive patterns of coping amongst student veterans experiencing common triggering events when transitioning to postsecondary education.

Bridges (2004) posits that all transitions "begin with endings" as previous ties are severed or these shared experiences end in one process in order for a new beginning or some sense of normalcy to occur. The ripple effect of a new beginning can permeate into biological, psychological, and social aspects of the individual as they reflect on their current circumstances when relating to individuals within their corresponding environment. In this study, a significant number of student veterans (45\%) considered their transition to be abnormal compared to their peers. This would confirm previous study findings that highlight the nontraditional aspects of being a student veteran (Ackerman et al., 2009; Daly \& Garrity, 2013; DiRamio et al., 2008; Elliott, Gonzalez, \& Larsen, 2011; Griffin \& Gilbert, 2015; McBain et al., 2012; Rumann \& Hamrick, 2010). Internalized stress associated with age differences and life experiences create a juxtaposition for building new relationships, confirmed in open-ended reports in this study. The inequity in life experiences perceived by student veterans creates a perceived barrier to engage in communication and openly discuss topics that may not be relevant or considered valued by the academic culture. This highlights the tendency for student veterans to socially isolate from the average student body or congregate exclusively with other student veterans for social support. This finding is more representative of avoidant, emotion-based styles of coping as it pertains to developing new relationships in the postsecondary context (Snyder \& Pullers, 2001; Suls \& Fletcher, 1985). Further inquiry into the coping assessment styles of student veterans when building new relationships is warranted as many veterans resort to negative avoidant coping strategies such as alcohol 
and nicotine use in order to manage stress (Bray, Fairbank, \& Marsden, 1999; Jacobson et al., 2008; Larsen \& Norman, 2014). The notion of closing the door on their previous self makes the transition more difficult as they look for a new way to construct relationships within the postsecondary context.

The most significant finding in this study pertained to the limited use of institutional support services by veterans. Student veterans reported lack of seeking support provided by the university and did not use the local VRC by a wide margin of $62 \%$. This would further confirm that a high percentage of student veterans in this sample preferred autonomous methods of coping with transition stressors. Also, they preferred to use their own skills and attributes learned from the military to solve problems experienced within the transition process. The findings attributed to healthy coping strategies highlight the strengths of this sample and may suggest a positive influence on the community mental health initiatives provided in the military focused on developing coping strategies (Bowles \& Bates, 2010; Jarrett, 2007, 2013). This is an interesting finding as the transition to postsecondary education focuses on individualistic thinking and priorities contrary to military collectivistic culture.

Generally speaking, students require self-motivation and self-reliance in order to navigate the academic administrative processes and to successfully achieve learning based outcomes required for degree completion. However, student veterans who perceive alienation from their institution often struggle with academic requirements, express higher levels of stress related symptoms and socially isolate themselves (Elliott, Gonzalez, \& Larsen, 2011). Limited engagement and social participation may reflect the challenges of being a nontraditional student. For instance, managing family routines, 
engaging in work, and community activities can be difficult to balance as a full time student. Completing full time credit hours competes with available time required for family interactions and/or work schedule requirements needed to support a family. In these instances, student veterans often gravitate towards online educational programs as a rationalized alternative unaware that this method of learning achievement may not coincide with their specific learning style. Additionally, online educational programs offer limited support and resources for a student veteran that require counseling or disability support.

\section{Implications for Occupational Therapy Practice}

The first line of defense for enhancing or facilitating adaptive stress reactions would be to reinforce veterans' awareness of the transition and factors involved in adapting to the transition. This should occur as the veteran approaches their transition. Schlossberg (1981) posits, "adaptation to transitions are affected by a specific set of variables such as role change, affect, source timing, onset, duration, and the degree of stress" (p. 8). Occupational therapists working with active military service members must facilitate an appraisal and awareness of these variables when a postsecondary education transition is anticipated. Occupational therapists should start by developing partnerships with local military transition assistance programs in order to assess current programs, partnerships, and resources in order to optimize services and offer any transition supports that would augment gaps in services currently provided to veterans.

Education on common challenges experienced in individualistic social environments is warranted as a civilian for the military cultural environment is collectively-oriented. Both military service members separating from the military and 
newly enrolled student veterans must understand the differences in how support is provided for challenges experienced in the individualistic social environment versus collectivistic social environment. They also need guidance on what entitlements they may receive for their particular life circumstances. Additionally, self-awareness of their current coping style can be screened using simple inventories such as the Ways of Coping Checklist-Revised (Penley, Tomaka, \&Wiebe, 2002) in order to identify their individualized coping style. Understanding their current coping style will provide guidance in how the individual perceives stressors in the civilian and/or postsecondary education environment and provide insights into which active coping strategies may be best to adopt or develop. Occupational therapists performing these screenings may also perform consultations and client education on remedial life skills and social skills training. Development of individualized coping strategies in conjunction with life skill development is needed for the acculturation process as veterans clearly experience problems with adjusting to college culture and with new habits, roles, and routines following active military duty.

The endorsement of autonomy in coping with stressful circumstances can be an empowering client factor for occupational therapists familiar with stress management training or individualized life skill development. Veterans who exhibit a strong sense of autonomy and intrinsic motivation may be exposed to education that enhances mental functions used in daily occupations. Furthermore, student veterans expressing high autonomy serve as ideal participants in coping strategies focused in creative writing, (Esterling, L’Abate, Murray \& Pennebaker, 1999; Progoff, 1992) creative expression, (Samuels, 1995) relaxation exercises in deep breathing and progressive muscle 
relaxation, (Lopata, Nida, \& Marable, 2006; Rausch, Gramling, \& Auerback, 2006) and mindfulness-based stress reduction (Davidson et al., 2003; Grossman, Niemann, Schmidt, \& Walach, 2004; Kabat-Zinn \& Chapman-Waldrop, 1988; Kabat-Zinn, 1994, 2003). In addition, education provided in formats such as web-based learning, tele-health education, and electronic based applications for smart devices would be optimal. Occupational therapists knowledgeable in these electronic tools would facilitate timeefficient and cost-effective measures for autonomous individuals with reportedly low levels of stress and adjustment concerns. Also, occupational therapists would expect this population to incorporate coping strategies into daily use with relative ease.

Many veterans with functional disabilities in postsecondary education may require intensive counseling support. Occupational therapy evaluations utilizing occupation-based methods of assessment can be instrumental in assessing the veteran's goals and acceptance of the transition. Assessments such as the Canadian Occupational Performance measure, (Law et al., 1990) the Model of Human Occupation Screening Tool, (Parkinson, Forsyth, \& Kielhofner, 2004) and the Occupational Case Analysis Interview and Rating Scale (Kaplan \& Kielhofner, 1989) can be instrumental in quantifying subjective experiences and clinical observations needed to guide intervention strategies for coping; reflective of veteran challenges with dysfunction and transitioning. According to the Department of Veterans Affairs Epidemiology Program report (2011), $52 \%$ of combat veterans have received one or more mental health diagnoses and $28 \%$ have received a diagnosis of Post-Traumatic Stress Disorder (PTSD). Student veterans representative of these epidemiological estimations may require screening and/or intervention for active mental health symptoms and individual or group intervention. 
Occupational therapy educators must liaise with university counseling services to form a unified front in supporting the psychosocial needs of student veterans. The facilitation of preparatory activities and interventions such as motivational interviewing are designed to evoke engagement in mental health care, especially for veterans perceiving barriers or stigma to seeking care. Motivational interviewing (MI) has been demonstrated as an effective intervention for preventative health programs (Lundahl, Kunz, Brownell, Tollefson, \& Burke, 2010) and for mental health engagement (Seal, Abadjian, McCamish, Shi, Tarasovsky, \& Weingardt, 2012). MI may be used to promote coping skill development and support self-efficacy in disabled veterans.

Advocacy and outreach for student veterans should remain a priority for providers, educators and university administrators alike. Not only do these efforts support veteran needs but, it also supports the retention strategies of the university. As evidenced by this study, student veterans are reluctant to self-identify and seek out support programs provided by institutions. Universities must coordinate programs and resources in order to centralize processes for orientation and acculturation of student veterans. Universities that devote resourcing to a centralized Veteran Resource Center provide a unified location with high potential for veteran performance skill and pattern development. These facilities provide a supportive environment occupied by a group of student veterans with similar experiences of transition to postsecondary education. The importance of Veteran Resource Centers have been identified in numerous studies (Cook \& Kim, 2009; Daly \& Garity, 2013; McBain et al., 2012). However, administrative support required to guide veterans through the Post 9/11 GI Bill process varies at each institution. Consolidating staff into a centralized point of reference such as a Veterans 
Affairs Office certifying official, university counselor and Veteran Resource Director may provide an effective team for responding to veterans' needs, planning out-reach initiatives, and administratively tracking student veterans for retention. Occupational therapy educators may provide consultations with university officials developing Veteran Resource Centers and identify critical areas that occupational therapy can support student veteran occupations. These efforts could help diminish the major sources of distress reported by student veterans in financial, administrative and psychosocial burdens and support the university's overarching strategic priorities.

\section{Limitations}

This pilot study must be interpreted in light of several limitations. The low response rate, albeit common for veteran populations comprised mostly of young unmarried males (Eaker et al., 1998; Iversen et al., 2006; Tolonon et al., 2006), limits the generalizability of results beyond the sample studied. The use of an online survey design was chosen by the authors to reach student veterans that often do not routinely use the local VRC. However, paper surveys administered in face-to-face settings demonstrate the most reliable method for increasing response rates (Nulty, 2008). Ten of the initial respondents failed to complete the entire survey, which highlights two concerns regarding, (1) placement of survey items and (2) the perception of usefulness of the survey. For instance, the authors chose to place the demographic questions of the survey toward the end of the survey as recommended by our established procedures, (Dillman et al., 2009) however, placing demographic questions at the beginning of a survey can improve item response rates in veteran studies (Teclaw, Price, \& Osatuke, 2012). Likewise, survey researchers recommend that student respondents must see usefulness in 
survey outcomes in some form of substantiated "follow-up" that valuates their response (Thompson \& Surface, 2009).

The decision to include student veterans no greater than 3 years removed from combat service limited a high proportion of potential respondents. Also, this study only collected descriptive statistics which cannot be used to provide any inferences on the causality of adaptive stress behaviors that demonstrate an outcome on reducing perceived stress. The data can only be used to describe the population studied at this single large research intensive institution. Despite the limitations identified in this study, the novel survey development strategy extends the body of literature on transition stress identification for student veterans. This approach may assist providers and researchers in developing a rating scale to identify transition stressors experienced in student veterans and wounded warriors. Further research is required on preventative health programs and interventions that assist student veterans in transitioning to postsecondary education.

\section{Conclusion}

The experiences and challenges for student veterans transitioning to postsecondary education can be comprehensively described utilizing Anderson, Goodman, and Schlossberg's conceptual framework on Adult Transitioning. The unique life experiences of student veterans exposed to combat and military culture provides a complex reality that can be observed from a holistic lens. Student veterans may see their transition as an anticipated, unanticipated, or non-event that can be feasibly understood through the Adult Transitioning model. This study demonstrates that the transition model illuminates student veterans' perceptions on the intersection of the individual, environment, and transition experience. Further studies addressing the complex 
phenomenon of transitioning from the military context to postsecondary education are required in order to build an adequate description of all three facets for predictive and prescriptive study. The findings of this pilot study reinforce the results of larger existing studies and adds an examining lens for coping in Schlossberg, Waters, \& Goodman's (1995) Adult Transition model. The Transition framework proves to be ideal in developing a coping factor line of inquiry for empirical study in life events and occupation.

\section{This chapter has been accepted for publication:}

Gregg, B.T., Kitzman, P.H., \& Shordike, A. (2016). "Well-being and coping of student veterans readjusting into academia: A pilot survey." Occupational Therapy in Mental Health, in press. Copyright (C) 2016, Taylor \& Francis.

Copyright @ Brian Tuan Gregg 2016 


\section{Chapter Four: Experiences of Veterans Transitioning into Academia Introduction}

More than 500,000 military veterans returning from conflicts in Iraq and Afghanistan have transitioned to civilian life by enrolling in postsecondary education (McBain et al., 2012). In fact, public institutions have recorded an average increase of 156 to 370 veteran enrollments per institution (McBain et al., 2012), with enrollment rates projected to increase as the Department of Defense downsizes (Ellison et al., 2012). Combat veterans attending postsecondary institutions bring an abundance of life experience to the educational context, however, 44 percent of veterans report difficulty transitioning to civilian life following combat service (Morin, 2011).

Veteran transition studies focus on the experience of reintegration into the civilian community following active duty service, and often highlight factors that veterans perceive to influence the "seamlessness" of their transition (Quillen-Armstrong, 2007; Stiglitz \& Blimes, 2008, p. 68). For instance, one transition study of over 1,800 veterans surveyed found, 48 percent experienced strains on family life, 47 percent experienced outbursts of anger, 49 percent experienced posttraumatic stress, and 32 percent experienced occasional loss of interest in daily activities (Morin, 2011). In the same study, predictors representing a "harder time" for veteran re-entry into civilian life included: (1) experiencing a traumatic event, (2) being seriously injured, (3) married while serving as a Post-9/11 veteran, (4) being a Post 9/11 veteran, (5) serving in combat, and (6) knowing someone killed/injured (Morin, 2011). The results of this study suggest a long term consequence for engagement in a wartime context, which highlights the signature cognitive and behavioral injuries (e.g., Traumatic Brain Injury, Post-Traumatic 
Stress Disorder, anxiety or depressive symptoms) of the second gulf war. These prolonged cognitive and behavioral consequences are commonly labeled as the "invisible wounds" of war (Tanielian \& Jaycox, 2008).

Most veterans transition from the military to civilian life without the manifestation of pathological problems. However, veterans who do experience difficulties with their transition most notably exhibit psychosocial stressors that affect their social interactions, social participation, and daily self-care. For example, Sayer et al. (2010) conducted a national survey of over 750 Gulf War II veterans receiving Veterans Affairs (VA) medical care to investigate the prevalence of community reintegration problems. Analysis of the veteran responses identified perceived deficits in social functioning and social participation when reintegrating or transitioning to civilian life. In fact, 25 to 56 percent of veterans reported difficulty in the domains of social functioning, productivity, community involvement, and self-care. Also, 57 percent reported anger issues and 31 percent reported increased alcohol and/or drug use one year after reintegration to civilian life (Sayer et al., 2010).

Understanding veteran transitions to postsecondary education begins with the perceptions of veterans feeling "underprepared" for academia (Ackerman et al., 2009; Ryan et al., 2011). Veterans' feelings of poor preparation stem from a perceived loss of time away from academia while serving in the military (Ackerman et al., 2009). The concerns of academic failure in underprepared veterans are realistic as veterans enrolled in four year institutions often fail to graduate or take longer to graduate, with attrition rates ranging 46-88\% (Cate, 2014; Wood, 2013). Veterans transitioning to postsecondary education also experience difficulty coping with the psychosocial effects of war and 
"fitting-in" on campuses (Ackerman et al., 2009). For instance, a common theme reported in student veteran studies is the challenge of socially connecting or belonging with the university and/or other students (Ackerman et al., 2009; DiRamio et al., 2008; DiRamio \& Spires, 2009; Jones, 2013; Olsen, Badger, \& McCuddy, 2014; Rumann \& Hamrick, 2009; Vance \& Miller, 2009).

DiRamio, Ackerman, and Mitchell (2008) offer a theoretical foundation for explaining how student veterans cope with transitions by a process of moving into the military, through deployments, out of the military, and back into education. The results of their theoretical study suggested veterans experience issues with "relearning study skills, connecting with peers, and dealing with financial concerns" and require a transition coach to serve as a holistic liaison between the veteran and the university (DiRamio et al., 2008). The coping process is multifaceted and further complicated when veterans enter postsecondary education with a disability. Limited research has been performed on wounded warriors enrolled in postsecondary education, though in a crosssectional study, Vance and Miller (2009) found that the majority (90 percent) of disabilities experienced by student veterans consisted of psychological, mobility, and learning disabilities.

The intersection of occupation and veterans in transition has been explored in a study by Plach and Sells (2013), examining the perceived problem areas young veterans experience when reintegrating into the community. In this cross-sectional study of 30 veterans, the researchers screened for mental health and substance abuse disorders while simultaneously administering the Canadian Occupational Performance Measure (COPM; Law et al., 2005). The two major findings of this study reported perceived problem areas 
in occupational domains of leisure and productivity, as over 70 percent of veterans reported difficulty with (1) relationships (e.g. socially connecting/relating to people outside of the military environment) and (2) transitioning to the student role (e.g. relating to students and having requisite skills for academia) (Plach \& Sells, 2013).

Tomar and Stoffel (2014) performed the second occupational exploration of student veteran transitions to postsecondary education using the participatory action research method, Photovoice (Wang \& Burris, 1997). In their exploration of two student veterans transitioning to postsecondary education, Tomar and Stoffel sought to understand the veterans' lived experiences and factors influencing their educational goals through the analysis of narratives and collected photographs that held meaning to each veteran. The results of their study identified themes that presented a trajectory of life experiences influenced by context and temporal factors such as the reminiscence for military experiences, transition to student life, entry into a new life stage, and influences from the university environment (Tomar \& Stoffel, 2014).

The body of occupational therapy research describing veterans' transitions to postsecondary education is limited in scope, which is of particular concern given the anticipated growth of veterans entering postsecondary education to develop skills for civilian life (Ackerman et al., 2009; Church, 2009; Clemens \& Milsom, 2008; Zinger \& Cohen, 2010). Considering the anticipated growth of the student veteran population and limited descriptive research available describing their lived experiences when transitioning to postsecondary education from an occupational perspective, the researchers of this current study decided to examine the lived experiences of student veterans transitioning to postsecondary education. Therefore, the purpose of this 
qualitative phenomenological study was to explore the lived experiences of discharged combat veterans transitioning to the occupation of education.

\section{Methods}

\section{Research Design}

The researchers of this study utilized a qualitative phenomenological study design to answer the grand question of, "what are the lived experiences of discharged combat veterans transitioning to the occupation of education?" Table 4.1 summarizes our process of formulating interview questions based on the research questions to explore the lived experiences of student veterans. Qualitative research empowers participants to share their stories and voices on experiences and contextual factors that cannot be analyzed by conventional statistical methodologies (Creswell, 2013). Phenomenology was chosen for this study as it describes the lived human experiences based on the principle that "scientific knowledge begins with unbiased descriptions of the subject matter" (Polkinghorne, 2005; Wertz, 2005). Since the majority of the psychosocial experience is not directly observable (i.e., how student veterans interpret their role or the support of the environment), the participants' textural descriptions of their individualized subjective experience provided insightful evidence that would have been unattainable from quantitative methods. This study was approved by a university Institutional Review Board and all participants provided informed consent. All research took place at a large land grant university in the southern region of the United States. 
Table 4.1. Grand Research Question, Sub-Research Questions, and Interview Questions

\begin{tabular}{|c|c|c|}
\hline Grand Question & Sub-Questions & Interview Questions \\
\hline $\begin{array}{l}\text { What are the lived experiences } \\
\text { of discharged combat veterans } \\
\text { transitioning to the occupation } \\
\text { of education? }\end{array}$ & $\begin{array}{l}\text { - What reasons do combat } \\
\text { veterans report for using their } \\
\text { Post } 9 / 11 \text { GI Bill? } \\
\text { - Is social support used by } \\
\text { combat veterans transitioning } \\
\text { to education? If so, how? } \\
\text { - What are combat veterans' } \\
\text { attitudes towards the military } \\
\text { as they transition to } \\
\text { education? } \\
\text { - What do combat veterans } \\
\text { think about the relationships } \\
\text { with their peers? } \\
\text { - What are combat veterans' } \\
\text { attitudes when they transition } \\
\text { to education? }\end{array}$ & $\begin{array}{l}\text { - In general, how would you } \\
\text { describe your experience of } \\
\text { transitioning from the military } \\
\text { to school? } \\
\text { - How would you describe your } \\
\text { communication with family } \\
\text { and friends when transitioning } \\
\text { to school? } \\
\text { - What emotions did you } \\
\text { experience as you transitioned } \\
\text { from school? } \\
\text { - How would you describe your } \\
\text { "role" or "position" now that } \\
\text { you are taking classes? } \\
\text { - How satisfied are you with } \\
\text { your current role? } \\
\text { - What do you value most of } \\
\text { your transition experience? } \\
\text { - What did you value least? } \\
\text { - Were your expectations met? } \\
\text { Why or why not? } \\
\text { - Were there any hardships that } \\
\text { occurred in your transition to } \\
\text { school? If so, how did you } \\
\text { manage? } \\
\text { - What forms of support have } \\
\text { you received in your transition } \\
\text { to school? } \\
\text { - Do you identify yourself as a } \\
\text { non-traditional student? If so, } \\
\text { why? } \\
\text { university a "Veteran friendly } \\
\text { campus? Why or why not? }\end{array}$ \\
\hline
\end{tabular}




\section{Recruitment}

The researchers set out to achieve a purposive sample comparable to the active military population with $85 \%$ male and $15 \%$ female (Military Onesource, 2012). Student veterans met inclusion criteria for the study if they were: 1) actively enrolled in the university, 2) between the ages of $20-45,3$ ) no more than 5 years removed from a combat tour, and 4) able to speak, write, and understand the English language. The researchers established cut-offs for combat military service (within the past 5 years) to maximize the number of participants with the most recent military experiences affecting the current transition. Any participant meeting inclusion criteria was considered for enrollment in the study, regardless of gender or ethnic background. Participants were excluded if they were currently serving as an active duty service member.

Participants were recruited through multiple means. Electronic mail notifications were sent through a university Veterans Resource Center (VRC) Listserv and recruitment posters were displayed at the VRC. Volunteers who had completed a previous pilot survey study conducted by the primary investigator were also contacted by electronic mail notifications to discuss interest in this study. Finally, snowball sampling was incorporated by giving recruitment flyers to interview participants and requesting that these participants deliver the flyers to other student veterans interested in participating. This method was instrumental in reaching student veterans who did not utilize the VRC and for those who routinely concealed their veteran status in order to "blend in" (DiRamio et al., 2008). All eligible participants were approached with a description of the purpose of the study and provided an opportunity to clarify any questions. 


\section{Data Collection}

Semi-structured interviews were conducted by the first author to collect participant responses regarding their transition experience from the military to postsecondary education. The interviews lasted up to 52 minutes. The interview questions were followed by a prompt to encourage a rich descriptive response. Examples of a prompts included, "Can you describe that more?" or "How did you feel about that?" The interviewer avoided the use of leading questions. Each question, prompt, and/or paraphrase attempted to facilitate the participants' description of their personal experiences. Each interview was audio-recorded and transcribed verbatim. Immediately following each interview, the interviewer recorded observations and reflections of the encounter as a field note. The field notes were used to identify meaningful topics and concepts covered that could be explored in follow up interviews with each participant during member-checking. Sampling and data collection ceased upon reaching data saturation. Saturation refers to a reference point in which the researcher is no longer hearing or observing novel information during the interviews (Patton, 2002). The final participant sample demonstrated a robustness consistent with valid phenomenological inquiry (Creswell, 2013). All data collected was kept confidential and pseudonyms were given to participants in order to de-identify their responses.

\section{Data Analysis}

The researchers modified the phenomenological thematic analysis procedures by Braun and Clarke (2006) to include an overarching "essence" that embodied the emergent themes described by student veterans in transition. The steps incorporated were as follows: (1) read interview transcriptions multiple times, (2) identified significant 
statements (3) developed initial codes, (4) reviewed initial codes in relation to emerging themes, (5) named and described themes and (6) developed an essential meaning that summated emergent themes. In short, as the themes and sub-themes emerged the researchers reviewed the textural descriptions in order to identify an overarching composite meaning for the transition experience. According to Braun and Clark (2006), "all aspects of a theme needs to cohere around a central idea or concept." These procedures were essential in accurately analyzing the phenomenological data and providing a structural flow and interconnectedness of the data. The primary researcher was guided by an expert qualitative researcher in peer debriefings to check the interview protocol, interview technique, coding, and interpretive analysis of themes. Patterns across transcripts were used to identify common and reoccurring themes which, were collectively organized using word processing and the qualitative software Nvivo $10^{\mathrm{TM}}$ (QSR International, 2014).

\section{Rigor}

The researchers used strategies described by Lincoln and Guba (1989) throughout the data analysis process to enhance the rigor or trustworthiness of the qualitative findings. A bracketing strategy in the form of reflexive journaling was employed prior to each interview session and throughout the analytical process. Bracketing is defined as “suspending the researcher's own beliefs to keep him/her as open as possible to what research participants want to share" (Polkinghorne, 1989). The primary researcher was on active duty military service and was himself a student in a postsecondary educational setting. As a result of these experiences, he came to the research project with preconceptions and presuppositions about the expected process of veterans transitioning 
to academia. In addition to bracketing, he discussed his biases with the second author during weekly data analysis peer debriefings. Member-checking was implemented once all interviews were completed to clarify the interpretations of the researcher. An audit trail was maintained via researcher memos that were annotated during analysis to make associations between codes to emerging themes.

\section{Results}

Table 4.2 summarizes the participant characteristics for this study. All thirteen of the participants were undergraduate students. There were nine males and four females with all but one participant, of Caucasian ethnicity. Seven participants were active in the local university Student Veterans Association (SVA) and twelve endorsed active engagement with the university VRC.

Table 4.2. Participant Characteristics

\begin{tabular}{|c|c|c|c|c|c|c|c|c|c|}
\hline Pseudonym & Gender & Age & Ethnicity & $\begin{array}{l}\text { Service } \\
\text { Branch }\end{array}$ & $\begin{array}{c}\text { Military } \\
\text { Component }\end{array}$ & \# Dep & $\begin{array}{c}\text { Dep } \\
\text { Length* }\end{array}$ & VSA & VRC \\
\hline Andre & M & 28 & Caucasian & Army & A & 1 & 15 & $\mathrm{Y}$ & $\mathrm{Y}$ \\
\hline Andy & M & 22 & Caucasian & Army & NG & 1 & 13 & $\mathrm{Y}$ & $\mathrm{Y}$ \\
\hline Brad & M & 29 & Caucasian & Marines & A & 2 & 12,6 & $\mathrm{Y}$ & $\mathrm{Y}$ \\
\hline Evan & $\mathrm{M}$ & 27 & Caucasian & Army & $\mathrm{NG}$ & 1 & 9 & $\mathrm{~N}$ & $\mathrm{Y}$ \\
\hline James & M & 24 & $\begin{array}{l}\text { African- } \\
\text { American }\end{array}$ & Marines & A & 1 & 7 & $\mathrm{~N}$ & $\mathrm{Y}$ \\
\hline Jason & $\mathrm{M}$ & 27 & Caucasian & Army & $\mathrm{R}$ & 1 & 12 & $\mathrm{Y}$ & $\mathrm{Y}$ \\
\hline Jenna & $\mathrm{F}$ & 27 & Caucasian & Army & A & 2 & 15,12 & $\mathrm{Y}$ & $\mathrm{Y}$ \\
\hline Kevin & M & 29 & Caucasian & Army & A & 1 & 11 & $\mathrm{Y}$ & $\mathrm{Y}$ \\
\hline Kelly & $\mathrm{F}$ & 32 & Caucasian & $\begin{array}{c}\text { Air } \\
\text { Force }\end{array}$ & A & 2 & 5,9 & $\mathrm{~N}$ & $\mathrm{Y}$ \\
\hline Larry & M & 25 & Caucasian & Army & A & 2 & 12,9 & $\mathrm{~N}$ & $\mathrm{Y}$ \\
\hline Mike & M & 28 & Caucasian & Marines & A & 1 & 5 & $\mathrm{Y}$ & $\mathrm{Y}$ \\
\hline Sara & $\mathrm{F}$ & 32 & Caucasian & Army & $\mathrm{NG}$ & 1 & 12 & $\mathrm{~N}$ & $\mathrm{Y}$ \\
\hline Tina & $\mathrm{F}$ & 25 & Caucasian & Army & NG & 1 & 7 & $\mathrm{~N}$ & $\mathrm{~N}$ \\
\hline
\end{tabular}


Table 4.3 provides an example of how participant data was coded. We started with 357 significant statements that were categorized into 18 initial codes. The 18 codes were compared and contrasted and further organized into three themes, six sub-themes, and an overall essence describing the transition to postsecondary education. Through the repeated readings by the researcher and peer debriefings with the expert qualitative researcher, an essential meaning of 'emerging in college culture' was constructed from the themes which described the emergent experiences of the student veterans. Emerging in college culture represented a transaction process of sorting military experiences, skills, and relationships needed for developing a new identity filled with purpose and meaning.

Veterans in this study perceived themselves as nontraditional students with unique differences and challenges compared to their peers and expressed difficulty relating to others as they navigated the postsecondary education context. Student veterans sought out other veterans for support and to get connected with local university veteran resources in essence to, 'emerge in college culture' as best as possible. The core themes that emerged from the data included (1) repurposing military experiences for civilian life, (2) reconstructing civilian identity, and (3) navigating postsecondary context and interactions. Each theme is described below, with supporting participant statements. 
Table 4.3. Example Participant Statements for Themes and Sub-themes

\begin{tabular}{ccc}
\hline Themes \& Sub-themes & Initial Coded Category
\end{tabular}

Theme 1: Repurposing Military Experiences for Civilian life

Moving on From Military Frustrations $\quad$ Rushing the military separation

Finding Veterans Who Could Understand

Being attached to military culture

Theme 2: Reconstructing Civilian Identity

Feeling Out-of-place

Reflecting on emotions of starting over

Re-defining aspects of the individual

Projecting "Self" as a Professional
Taking control over new responsibilities

Ijust wished that the military would prepare us a little bit better for stuff like this. When it is time to get out of the service, they throw so much information on you at the last week and at that point you are ready to just get the heck out of there.

As soon as I got out, I joined a Marine Corps league. And I ran into Marines so, uh, the Veteran thing helped me a lot. Cause they had already been there year or two and already figured out how to handle it.

My transition to being around college age students was difficult because you don't know anybody and you don't relate to anybody. So, its like you are this loner, kind of floating around campus and whatnot.

It's a little bit different. Instead of having to worry about what someone is doing or getting them prepared you have to focus on yourself. So, it's just a little bit odd I guess, at first, focusing on yourself.

Definitely that self-dependence. Just learning that process of self-motivation.

Theme 3: Navigating Postsecondary Context and Interactions

Adapting to a New Context

Navigating postsecondary education culture

Difficulty Relating to Students
Learning to relate with students
Navigating the bureaucracy was a little bit of a challenge, uh, rather than having things spoon fed to you basically, you now had to figure things out on

It's just a little weird at first fitting in with 18 and 19 year olds when you are nearly thirty. 


\section{Theme 1: Repurposing Military Experiences for Civilian Life}

The participants described their transition as a process of repurposing what they learned in the military to benefit their current life as a student. Participants used skills and competencies attained during their military career to enhance their daily life in the educational context. For many, this process required a reflection of both the positive and negative experiences of the military. For example, Jason described how he used a form of controlled aggression and tactical competency that he learned in military training for combat operations to guide how he approached being motivated as a student:

I took those principles that I learned in the military and that discipline and that violence. I had to use that violence on my mentality. I had to have a violent and aggressive mentality that, [no], you have to get this done. No one else is going to do this for you. There is no one over your shoulder. There is [no one] next to you now to push you forward. You are going to have to do that.

Participants described the importance of discipline learned during their military career and how helpful it was for the educational context. Brad stated:

If I had one thing it would be discipline. Because I was already used to showing up at 0800 [in the morning]. And I show up every day in class. I'm always ontime to class. I always bring everything I need. I always bring my books, my pencils, my computer, if I need it. Before the Marine Corps, I would not have had that. I would have been partying or never going to class or whatever. Now, I prepare the night before. I'm in class ready... That's the discipline the Marine Corps gave me. 
Participants noted that discipline learned in the military was essential for developing and maintaining a daily schedule that kept the individual focused on educational assignments. Jenna said:

And I think that is a remnant from the military. I am very disciplined with my time. Even like study hours are incorporated in there. So, I think those are the two main things that have translated. Just waking up early and keeping good track of my time. I think those are the two best parts left over from the military that have helped me the most.

Moving On From Military Frustrations. The participants described feeling that many elements of their military careers were deeply frustrating, and essential to move beyond in order to transition successfully to academia. For example, the collectivistic military culture emphasized a hierarchal social structure in which participants were asked to put the needs of their subordinates above their own. Participants described frustration with this aspect of military leadership. Jenna highlighted the difficulty she experienced being accountable for others in the military:

There is just so much about the Army that aspects of your life are kind of geared towards. Especially in a leadership position, I had to constantly ask myself, what might come up that might prevent my plans for this weekend. I can't even tell you how many times I had to leave parties or leave hospitals visiting people where one of my Soldiers got drunk and did something stupid. So, not to have like all these guys depending on me, weighing on my mind... I knew that there would be something different in school but, I didn't necessarily know what it was. 
I knew there would always be something to worry about but, it is not as grave as something happening to one of your guys.

Another participant, Jason, described his disenchantment following military sequestration, which limited his participation in military training:

So, I got very bitter and very angry with this institution (the Army). And I was like, I don't want any more of it. Cause, I had further goals and I had further dreams that I wanted to do in the military.

Larry described his positive feelings experienced in his transition from the military as he no longer had to suppress his individualized behaviors for the sake of collectivistic traditions within military culture:

I enjoy having freedom, having the ability to make my own decisions, craft my own schedule, and if, you know I could plan something 6 months ahead of time that I could actually stick to it come hell or high water. You know, instead of having everything be suspect and still little things, like not having to get a haircut, not having to shave my face every day. You know, the stuff that I despised while I was in the military, to be honest with you (laughter).

Finding Veterans Who Could Understand. The student veterans described the importance of finding other veterans that could understand the experience of transitioning to the educational environment. Building a new network of friendships with other veterans served as strategy for developing a social support system that resembled the military cultural environment. This support system was often used to cope with ongoing stressors related to previous combat experiences and to new situations experienced in the 
postsecondary education environment. Brad described his experiences of finding veterans in the following passage:

The first couple of times that I had conflicts, I was pretty upset and angry about it. And then that helped (talking to other veterans) me out... As soon as I got out, I joined a Marine Corps league. And I ran into Marines so, ah, the veteran thing helped me a lot. Cause they had already been there a year or two and already figured out how to handle it. So, we would talk about it. We would drink. We would go to the Marine Corps league and drink and talk about it. I just learned to not voice certain things... They don't, they (other students) don't really understand the inner fighting. They don't know. So, you kind of have to bottle it up. You can't talk about it so much. You have to find people that have kind of went through that same kind of thing. Especially, on a deployment.

Participants experienced the strength of military bonding and commitment for a collective purpose rather than an individual one. These bonds were deeply rooted and difficult to find in new friendships. Mike described it this way:

I think in the military that it is hard once you get out to find someone that you can trust just like when you were with your military brothers. And I think being around the veterans because you have been around a similar spot and been around the same stuff those kind of help to make it easier to get to know some of these guys cause a lot of these guys will never be as close to me as some of the guys that I served with but, they do everything they can to help me out.

Many participants were aware of the associations and organizational programs the campus employed to get veterans connected with others in the university. Mike felt this 
was worthwhile, but wanted it to be an active process of choosing to build new relationships:

I mean, there were the programs to help veterans get into it and we need those but, I think a lot of times we want to bring ourselves there. We don't want anybody to push us in that direction.

\section{Theme 2: Reconstructing Civilian Identity}

All of the participants highlighted the significance of their military career and persona, which gave them meaning and purpose in life. For instance, engagement in the military environment provided many opportunities for personal growth through professional accomplishments. The professional accomplishments culminated from individual and collective actions that resonated as meaningful experiences when each veteran reflected on the military career. Kelly described the significance of her military experiences and how it influenced her perception of the transition experience:

Ifelt like I had built so many accomplishments for myself in the service and I felt

like I was somebody and then whenever you start over and everything is brand new, I just kind of felt like I was lonely.

Transitioning to postsecondary education precipitated a life event that challenged how veterans perceived themselves, occupationally. Therefore, participants sought to reconstruct their military identity into a new civilian identity, which was a shift in their self-concept and perception of what was meaningful in their lives.

Many described this reconstruction process in terms synonymous to "doing and being," which referred to loss of a military role (doing) and learning to figure out who to 
"be" in civilian life. Jessica shared an example of the challenges females experience in reconstructing their civilian identity in these terms:

I took a lot of pride in what I did and... um, in my field was, I was a crew chief, mechanic on a Blackhawks and on hydraulics, aircraft hydraulics. And it was not a very, um, female oriented field and you know, I took a lot of pride in being able, being (1) accepted by my male counter-parts, which can be a challenge and (2) being not only good at what I did but, great at what I did, which was, was no more a reflection on me as my leadership. I had really great leadership along the way and [losing] that identity, was really difficult. No longer being able to say that ... that's who I was, was probably the hardest part.

Another participant, Jenna, also described reconstructing identity in terms of doing and being. Her example highlighted aspects of role reversal in reconstructing her civilian identity:

I used to be a platoon sergeant and it was like going from being the teacher, I was the role model, I was teaching the classes when we had down time. Then, transition from that to being in the background, not being up in front. And really seeing things from the perspective of my soldiers, you know. Trading roles entirely, going from teacher to student. So, that was a little difficult for me. So, it was totally different than what I was doing. That was what was hard about it, it was foreign.

Feeling Out-of-place. Participants also described the emotions that shaped their perception of the postsecondary context and the development of their civilian identity. The transition to being a student created challenges in forging new relationships, habits, 
roles, and routines as a nontraditional student who was older with different life experiences than the typical student. Forming these new occupational patterns, in the novel environment, and surrounded by younger students with less life experience seemed to be a difficult process for student veterans as described by Tina:

It was challenging at first because I felt very out of place. It was hard to feel that I was a part of the school. You know, the whole culture of going back to college. Just because you know you are different because you are ten years older than everybody else.

Another participant described how lonely she felt and the proactive measures she performed to feel more connected with the university culture:

I felt secluded so, I forced myself to be a part of the university. I forced myself to go to the veterans' resource center and ask them, can you please give me a list of organizations to be a part of. Through that I was bouncing around and it was kind of scary to show up to different organizations until I found something for me. Jenna succinctly summated the experience in the following statement:

I think that in the military, you underestimate, or maybe take for granted that you wake up every day knowing exactly, what you are going to wear, like how you have to have your hair and you know these steps. And it really is routine, [almost like], not to have that was the weirdest thing to me, at first. I had a lot of trouble of like just picking out what to wear. It's not like... it may be like a girly thing to say but, it was more related to the fact that for the past 7 and a half years I had put on the same thing. To take that away was bizarre at first. 
Projecting "Self" as a Professional. The participants described their approaches to educational experiences and how they strategically presented aspects of themselves as professionals. For the participants, it was important to be viewed as a student focused on completing educational outcomes regardless of their current life circumstances. The participants would compare and contrast themselves with traditional students and ensure that they projected personal traits of motivation, work ethic, and effective communication. For these reasons, they explicitly chose to present a professional demeanor when expressing their current self. An example of this was described by Larry:

I consider it my job now, because I am fortunate to get paid for it by the GI Bill um, because of that, I approach it like I would any other job. Show up on time, do my work, uh, I approach it in the professional sense, you know, maybe not dress or haircut, but in terms of my approach on my tasking or my duties for getting the best education that I can, and achieving the best, you know, GPA, something like that I can.

Jason's response echoed this position, "I guess my role or position would be a student that cares and tries to get the best grade in school that I possibly can versus my mom made me come to college." Sara further described the professional approach she took with professors in order to complete assignments in lieu of competing family situations: I have made an effort to speak to every one of my professors at the beginning of the semester and say, this is my situation, you know I'm 32 years old, I have a 4 year old at home, I own my home, I have animals I have to care for, you know. My husband, even before, he was a police officer, he worked third shift so, he 
wasn't always available and on the rare occasion where the you know where it

didn't matter how much I tried, there was no way for me to meet the requirement, I had the reputation with my professors that we were able to overcome that.

\section{Theme 3: Navigating Postsecondary Context and Interactions}

The participants described interactions that challenged their previous methods of communication or preferred communication styles. Social participation was difficult for the student veterans to engage in as they described situations in which a new lexicon, language, or a method for relating was needed in order to interact with the students and professors in their classes. Two sub-themes composed of "adapting to a new atmosphere" and "difficulty relating to students" emerged in relation to this process.

Adapting to a New Context. Participants described their need to adapt in order to navigate social interactions. The veterans reported needing to reflect after challenging social situations to identify strategies for future experiences. Kelly gave this example: I remember one of the first classes that I sat down in... you know in the first class when there are all those freshmen and it's big... what do you call it? Seating? And the girl sitting next to me is like "hi, what dorm are you in?" Um, I am not in a dorm. "You live off campus; that is so awesome!" You know, I don't know how to respond. I don't know what to say. I don't know how to talk about anything. I didn't know... How do you talk about anything in your life? I had no clue what to say to any students in the beginning. Like I didn't know... I would be like "so, what do you do for fun?" I didn't know how to have a conversation with anyone in the beginning. It took a while, but I figured it out. And if I were to say, "This happened to me, you know the snow so I had a two hour delay to get 
my daughter to school." They would be like, "you have a kid?" It's just, you don't really know how to converse.

Another participant described her discomfort with social interactions and how to communicate her current life circumstances. Her social responses reflected the attitudes, beliefs, and values developed in the military. Sara stated:

I didn't feel comfortable at all like having a conversation. Even people outside of school. Like when they say, "Hey what are you doing?" Well, I am going back to school. But I don't want to say that, because I am uncomfortable. I do not know why I say, "I am going back to school, I am a non-traditional student, prior military, and I am using my GI Bill." I always felt like I had to say that.

Difficulty Relating to Students. The biggest challenged described by all participants was the difficultly in relating to the traditional student or those typically between the ages of 18 to 22 . Differences perceived in age and life experience seemed to create a rift in how student veterans would approach social interactions and to whom they would continue to build relationships with. Being a nontraditional student heightened each veteran's awareness of differences existing in their social environment that strongly influenced their perceptions traditional student norms. An example of life differences experienced was provided by James:

I was married, had a 2 year old and was 22. Where most college kids are between eighteen to twenty-two. So, they were talking about prom, to where they went to high school, and what club they went to last night. You know, I am focused on school and going home to my wife and kids. And maturity levels; I was more focused on my teachers whereas, they are focused on the moment. 
Mike added to the life experience differences and alluded to the type of resource a veteran can be to the traditional student:

You know veterans that have been deployed have dealt with so much that people just don't understand. They just don't understand because they have not seen what you have seen or done what you have done. It's all a joke because they are looking at call of duty video games thinking that is combat. When really it's not. And most of these kids have never left their county. I think being veteranfriendly is helpful because there is so much that you can learn about a veteran if you just sit down and talk with them for a couple of hours. You will be amazed of some of the special stories that they have been through.

Jenna provided insights into why she could not relate to other students. They suggested that it is not just the difference in life experience or shared activities. Rather, they pose the problem lies in knowing how or learning methods to relate to traditional students with becoming maternalistic:

I don't feel like I relate in any way other than commiserating over the usual homework assignment; that's really our only common ground. It's not that I haven't tried to associate more with college students. It's just I really don't know how. Cause I don't really want to come across like a mother figure. I don't know. It's really a strange dynamic.

\section{Discussion}

This study aimed to describe the lived experiences of discharged combat veterans transitioning to the occupation of education. The study findings address a gap in the research regarding how student veterans perceived their transition and the factors that 
affected their occupational engagement in the postsecondary education context. Student veterans in this study described transitioning as a transactive process of standing in both worlds (military and civilian) that required making individualized decisions unfamiliar to them in a novel environment. The notion of focusing on individualistic aspirations highlighted a juxtaposition previously identified for veterans as it was a new experience that appeared contrary to the collectivistic aspirations promoted within the military context (Rumann \& Hamrick, 2010). The summation of veterans' transition experiences emerged as a process resulting from social interactions in college culture that also required navigation of academic processes in the university environment. Furthermore, this study highlighted how participants perceived and appraised their transition experience from an explicated veteran-centric cultural lens, which emphasizes the importance of examining student veteran occupational needs from a cultural perspective.

Durdella and Kim (2012) posit student veterans interpret their transition experiences primarily from a military cultural lens. In their study of student veteran status as a predictor for grade point average (GPA) and sense of belongingness, veterans reported more academic participation, interaction, and collaborative work than nonveterans, yet continued to attain lower GPAs than nonveteran students. Similarly, service members in the current study reported consistent use of their military cultural values and norms such as maintaining discipline, coordinating group projects, and exercising time management skills as strategies for facilitating academic participation in classes and collaborative groups. However, veterans in the current study perceived lower self-efficacy for general academic skills (reading, writing, mathematics) when comparing and contrasting themselves to nonveteran students. The current study did not address the 
effect of transitioning on student veteran GPA but, does seem to support the notion of veteran cultural values, beliefs, attitudes, and social cues affecting the learning experiences and social interactions with nonveteran students (Durdella \& Kim, 2012).

The transition experienced by student veterans in this study highlighted the challenge of reconstructing a civilian identity commonly identified in the literature (Ackerman et al., 2009; DiRamio et al., 2008; Elliott, Gonzalez, \& Larsen, 2011; Jones, 2013; Olsen, Badger, \& McCuddy, 2014; Rumann \& Hamrick, 2010; Tommar \& Stoffel, 2014). Interestingly, the reconstruction of identity began with student veterans reflecting on patterns of doing everyday tasks associated with previous military roles and collectively-based actions. Veterans ascribing meaning to aspects of their previous military self, described difficulty when making comparisons on their previous self to their current life circumstances. Therefore, becoming a student veteran with a newly constructed civilian identity, was aided by engagement in daily educational activities with other student veterans that could empathize with their transition experience.

Contrary to our expectations, participants reported the transitioning process as an overall positive experience that was challenging and "fearful" in the beginning but, became more manageable with the development of a strong support network consisting of family members and fellow student veterans. This was an encouraging finding as the body of literature suggests that veterans often avoid seeking assistance and support due to "high self-expectations" and the "hyper masculinity of military culture" (Caffrey, 2009; Tomar \& Stoffel, 2014; Vacchi, 2012). The development of veteran centers, veteran led student organizations, and peer mentoring has long been promoted within the literature (Ackerman et al., 2009; DiRamio et al., 2008; Elliott, Gonzalez \& Larsen, 2011; Griffin 
\& Gilbert, 2015; McBain et al., 2012; Rumann \& Hamrick, 2010) and seemed to resonate within the participants of this study who had access to a robust veteran resource center and active veteran community.

Another interesting finding in this study pertained to the incongruence between student veteran beliefs about building on their military experiences and how reluctant they were in sharing their military experiences in social interactions. For instance, student veterans ascribed significant meaning to their military experiences and wanting to build on these experiences by furthering their education with the use of their GI Bill entitlement. However, many student veterans would suppress aspects of their military experiences from their peers and would only engage in social interactions with other veterans. This paradox highlights a common problem student veterans have in relating to other traditional age students and internalizing the problem as a form of alienation perceived in civilian contexts (Ackerman et al., 2009; Ahern et al., 2014; DiRamio et al., 2008; Elliott, Gonzalez, \& Larsen, 2011; Griffin \& Gilbert, 2015; Holloway, 2009; Koenig et al., 2014; McBain et al., 2012; Rumann \& Hamrick, 2010; Tommar \& Stoffel, 2014; Vance \& Miller, 2009). However, it also highlights how susceptible student veterans are to socially isolating themselves from others due to preconceived notions of postsecondary education sociocultural norms. Durdella and Kim (2012) suggests student veterans may not be particularly receptive to civilian cultural cues within the educational context and require environmental support in promoting the quality of interactions and participation with nonveteran students and professors.

The development of veteran social support as student veterans navigated the postsecondary education context was an important finding as the interactions and 
relationships built with other veterans was a determinant of their perceived transition success. Also, the relationships with other student veterans were utilized to overcome daily obstacles and previous psychosocial burdens. Although the psychological status of participants in this study was not screened, many participants described repressed anger related to their combat experiences and noted the importance of their veteran support in overcoming the psychosocial problems incurred during their combat exposure. In sum, these findings indicate a need to support student veterans holistically in order to be responsive for their cultural specific challenges and for potential psychosocial problems that may hinder academic success (Ackerman et al., 2009; Ahern et al., 2014; DiRamio et al., 2008; Elliott, Gonzalez, \& Larsen, 2011; Griffin \& Gilbert, 2015).

\section{Limitations}

While this qualitative study demonstrated consistent themes that emerged across participant experiences, some limitations need to be discussed. First, heterogeneity likely exists at institutions in different regions with differing levels of resources, programs, and support devoted to veterans. Participants in this study were recruited from an institution with a vast amount of resources and support which, may impact their transition experiences compared to veterans attending institutions with limited veteran resources. Secondly, the majority of the student veterans interviewed within this study were comprised of a similar ethnicity. Only one participant identified as a minority. Differences in transition experiences may exist across veterans of differing racial and/or ethnic groups. Further research is warranted to look at the differences in the transition experiences between gender, culture, disability status, and educational status such as graduate students versus undergraduates and professional programs. Also, research is 
needed for longitudinal evidence explaining the transition process and occupational engagement.

\section{Implications for Occupational Therapy Practice}

Occupational therapy practitioners engage veterans in a variety of contexts such as hospital settings for inpatient and outpatient care, community mental health centers, workplaces, and within institutions of higher learning. Each of these environments has a cultural component that may require reflection prior to engaging veterans in the respective context. Occupational therapy practitioners and educators engaging veterans transitioning to postsecondary education require a culturally relevant lens to understand their unique experiences, which differs from the traditional student assimilating into the sociocultural norms of the educational environment (Ackerman et al., 2009; DiRamio et al., 2008; DiRamio \& Spires, 2009; Jones, 2013; Olsen, Badger, \& McCuddy, 2014;

Rumann \& Hamrick, 2009; Vance \& Miller, 2009). Occupational therapists must leverage the strengths of veterans to overcome institutional barriers that may exist and to facilitate pragmatic solutions for developing skills that foster academic success.

Effective skills in work, education, and social participation enable veterans to achieve a positive transition into civilian life and empowers self-sufficiency for leading an individualistically centered life; contrary to the interdependent military context. The findings of this study suggests the following implications for research and practice:

- Therapists working with injured or disabled veterans transitioning from the military should prioritize the assessment of psychosocial problems potentially impacting their current functional status. Culturally relevant approaches to occupational therapy evaluation exists, which may be used to identify and 
prioritize perceived psychosocial problem areas in the veteran's unique narrative or life flow (Iwama, 2006).

- Researchers have suggested the use of a transition coach for facilitating a holistic process addressing the transition needs of student veterans (DiRamio et al., 2008). Therefore, therapists may assess veteran perceived psychosocial problem areas with the Canadian Occupational Performance Measure (Law et al., 2005). This assessment may also prioritize the challenges of student veterans in a holistic process familiar to practitioners.

- Therapists working with veterans should also inquire about their educational status. As veterans are likely to use their Post 9/11 GI Bill to develop skills for civilian life, they would likely benefit from an assessment of their self-reported interest in specific vocations (Holland \& Messer, 2013) and current career thoughts (Sampson, Peterson, Lenz, Reardon, \& Saunders, 1996, 1998). Gathering a baseline understanding of their career interests may be used to discuss the skills and pragmatics of pursuing educational programs that align with their current ability.

- Veterans report positive transitions when interacting and connecting with other veterans, who are able to empathize with their current life circumstances. Therefore, therapists should facilitate occupations that promote social participation amongst veteran groups. The focus should be placed on getting veterans connected with one other in order to develop cohesive relationships similar to military culture that may broadly address tasks concerning veterancentric issues. 
- Educators should liaison with local university counseling centers and veteran resource programs to assess the feasibility or availability of veteran orientations, outreach programs, and/or remedial education courses. Veteran-specific programs are well received as they provide the formative experiences for connecting veterans with the university and each other. They also, present opportunities for health and wellness initiatives such as stress management education and activities that address coping strategies for transitioning to civilian life. Development of a program incorporating Matuska and Christiansen's (2008) model of "Life Balance" would be ideal to promote meaningful and sustainable occupational patterns as veteran transition. Use of the psychometrically reliable and valid, "Life Balance Inventory," could offer an outcome measurement demonstrating self-reported change from baseline for such an occupation-based program devoted to managing transition stress (Matuska, 2012).

\section{Conclusion}

The occupational needs of student veterans transitioning to postsecondary education is a national concern for occupational therapy practitioners and educators alike as the projected enrollment of this population is expected to increase to over 5 million veterans by 2020 (American Council on Education, 2014). As student veterans return from combat environments, the occupational therapy practice must be ready to guide these individuals through a culturally-sensitive process for reintegration and occupational engagement in the postsecondary education context. A culturally-sensitive approach is needed to address the historically high rates of mental health problems in veterans and military populations (Substance Abuse and Mental Health Services Administration, 2013) 
but, more importantly, to guide the post-traumatic growth (Larner, \& Blow, 2011; Tedeschi, \& Calhoun, 2004) and generalized occupational disruptions (Plach \& Sells, 2013; Tommar \& Stoffel, 2014) experienced by combat veterans in transition to the postsecondary education context. This study provides insights into the lived experience of student veteran transitions and how practitioners and educators can address veterans' needs from a military cultural lens.

\section{Portions of this chapter were accepted for publication in the:}

American Journal for Occupational Therapy.

Copyright (C) Brian Tuan Gregg 2016 


\section{Chapter Five: Student Veteran Occupational Transitions in Postsecondary Education: A Grounded Theory \\ Introduction}

Post 9/11 era United States veterans experience long term consequences in neurocognitive (Belanger, Uomoto, Vanderploeg, 2009; Polusny et al., 2011; Spelman et al., 2012), psychological (Pietrzak et al., 2009; Sayer et al., 2009), and social disorders (Brown, 2011; Cuber, 2012; Hinojosa et al., 2010; Sayers \& Farrow, 2009) following military overseas deployments and combat engagement. All of these challenges affect military veterans' mental health recovery across their life flow (Gregg, Howell, Quick \& Iwama, 2015) including re-adjustment to civilian professional and educational settings (Koenig et al., 2014). Consideration for Post 9/11 era veterans' re-adjustment in the postsecondary education context is of particular concern given the systematic downsizing of the Department of Defense (Ellison et al., 2012), anticipated growth of the student veteran population (McBain, Kim, Cook \& Snead, 2012), and the vulnerabilities in health problems veteran culture faces following deployment experiences (Anderson et al., 2003; Nayback, 2008; Ouimette et al., 2011).

Extant research on student veteran re-adjustments to postsecondary education uses the term transition to describe student veterans experiences when exiting the military and entering postsecondary education (Ackerman et al., 2009; DiRamio et al., 2008; DiRamio \& Spires, 2009; Jones, 2013; Olsen, Badger, \& McCuddy, 2014; Rumann \& Hamrick, 2009; Vance \& Miller, 2009). DiRamio, Ackerman, and Mitchell (2008) provide the seminal theory intended to explain how student veterans cope with transitions to postsecondary education. They posit student veterans transition via a process of 
"moving into the military, through deployments, out of the military, and back into education" (p. 80). DiRamio et al. (2008) provide a theoretical representation of student veteran transitions that applies a pre-existing coping model for life transitions (Schlossberg, Lynch, \& Chickering, 1989), suggesting veterans experience problems with remedial skills, interpersonal relationships, and managing finances. The need for a holistic transition liaison between the veteran and university supports is recommended (DiRamio et al., 2008), however, the cultural considerations of student veterans has not been explicitly addressed in student veteran theoretical investigations.

In fact, few descriptive studies exist that highlight cultural factors for Post 9/11 veterans in transition and the differences between civilian and military environments (Ames et al., 2011; Demers, 2011; Stachyra, 2011). Faulkner and McGaw’s (1977) model of reentry for Vietnam veterans theorizes transitioning in a three stage model of: disengagement from military life, re-entry into the civilian world after deployment, and full reintegration into civilian life. Koenig, Maguen, Monroy, Mayott, and Seal (2014) extended the theoretical research on veterans' re-entry to civilian life by examining the experiences of Post 9/11 era veterans and focusing on "complex social and temporal processes" (p. 415). Their findings identified sociocultural tensions between civilian and military social identities in cultural domains of intrapersonal, professional/educational, and interpersonal (Koenig et al., 2014), suggesting a similar "reverse culture shock" as seen with Canadian veterans (Westwood, Black, \& McLean, 2002).

The current theoretical literature explaining veteran transitions to civilian life and postsecondary education places a heavy emphasis on the proverbial self and individual actions. However, military cultural investigations that explicitly study individual and 
collective actions have not been investigated in student veteran transitions to postsecondary education. A broader interpretation of student veteran culture is needed as the western literature base on culture, health, and health care focuses on a highly contested concept in acculturation (Acevedo-García \& Bates, 2008; Carter-Pokras \& Bethune, 2009; Hunt et al., 2004; Kao, Hsu, \& Clark, 2004; Santiago-Irizarry, 1996; and Viruell-Fuentes, 2007). Studies on acculturation place an overt emphasis on individual actions, while "ignoring the effect of [collective factors] such as residence in lowresource communities, low socioeconomic position, the social construction of marked cultural identities, and institutional patterns of unequal treatment, all of which contribute to health disparities" (Zambrana \& Carter-Pokras, 2010, p. 21). Acculturation research also focuses on intra-individual traits, attitudes, values, and behaviors prevalent in public health literature. Therefore, a broad framework for examining veteran experiences of transitioning to postsecondary education culture was applied in this study to consider both individual and collective actions within societal institutions and collective strategies that affect student veterans' essential life flow (Iwama, 2006).

Given the historical challenges associated with the concept of transitioning for student veterans and the limited theoretical literature describing this concept in relation to veteran experiences in novel cultural environments, the purpose of this constructivist grounded theoretical study was to intensively interview student veterans attending fouryear postsecondary educational institutions. Data collected was constantly-compared to co-construct an emergent conceptualization of the individual and collective student veteran transition experiences. The grand tour question for this study was, "What is the 
meaning of the transition experience to postsecondary education for student veterans? A series of sub-questions explored included:

- How do student veterans describe their current physical and social environment?

- What life circumstances shape student veterans' transitions to postsecondary education?

- How do individual assets and liabilities influence student veterans' transitions to postsecondary education?

\section{Methods}

\section{Study Design}

The research study design employed was a Constructivist Grounded Theory (CGT) approach described by Charmaz $(2006,2014)$ utilizing the Kawa (Japanese for River) model "a priori" as a culturally-relevant guide for interviewer-participant discussions. CGT acknowledges the multiple realties research participants experience, which researchers use to construct an abstract theory interpretive of the social interactions between both the researcher and participant. This process of developing theory with explicit input from and interactions with research participants is known as "co-

constructing" theory, a hallmark of CGT (Charmaz, 2006, pp. 9-12). A qualitative study design was chosen as the best method available for exploring complex student veteran experiences transitioning to academia and the decision making process used to engage in university environmental supports and social interactions. Qualitative research empowers participants to share their stories and voices on experiences and contextual factors that cannot be analyzed by conventional statistical methodologies (Creswell, 2013). Use of 
the grounded theory methodology surpassed a description of participants' experiences and focused on co-constructing an abstract model that conceptualized those experiences (Creswell, 2013).

The Kawa model described by Iwama (2006) was chosen to gain a rich, graphic illustration of each veteran's narrative under inquiry with the use of a river metaphor. Developing Kawa graphic images facilitated an occupation-based process (Carmody et al., 2007) for interviewing, while enhancing rapport and self-empowering veterans to be key decision makers in their river (Humbert et al., 2014). The graphic images provided context to each veteran's transition experience and facilitated a reflective process for identifying meaning ascribed to each factor influencing veteran transitions. The Kawa model was also chosen for its epistemological underpinnings in social constructionism which posits that people construct social realties through individual and collective actions (Charmaz, 2006, p. 189). The incorporation of the Kawa model aligned well with CGT due to the shared philosophical underpinnings and ability to enhance intensive interviews used in CGT. Intensive interviews were designed to elicit participant descriptions and reflections on their experiences in methods that infrequently occurred in daily life (Charmaz, 2006, p. 25), which was also consistent with the use of the Kawa model. The Figure 5.1 demonstrates the study design and flow of participant recruitment for this study. 


\section{Figure 5.1. Study Design Schema.}

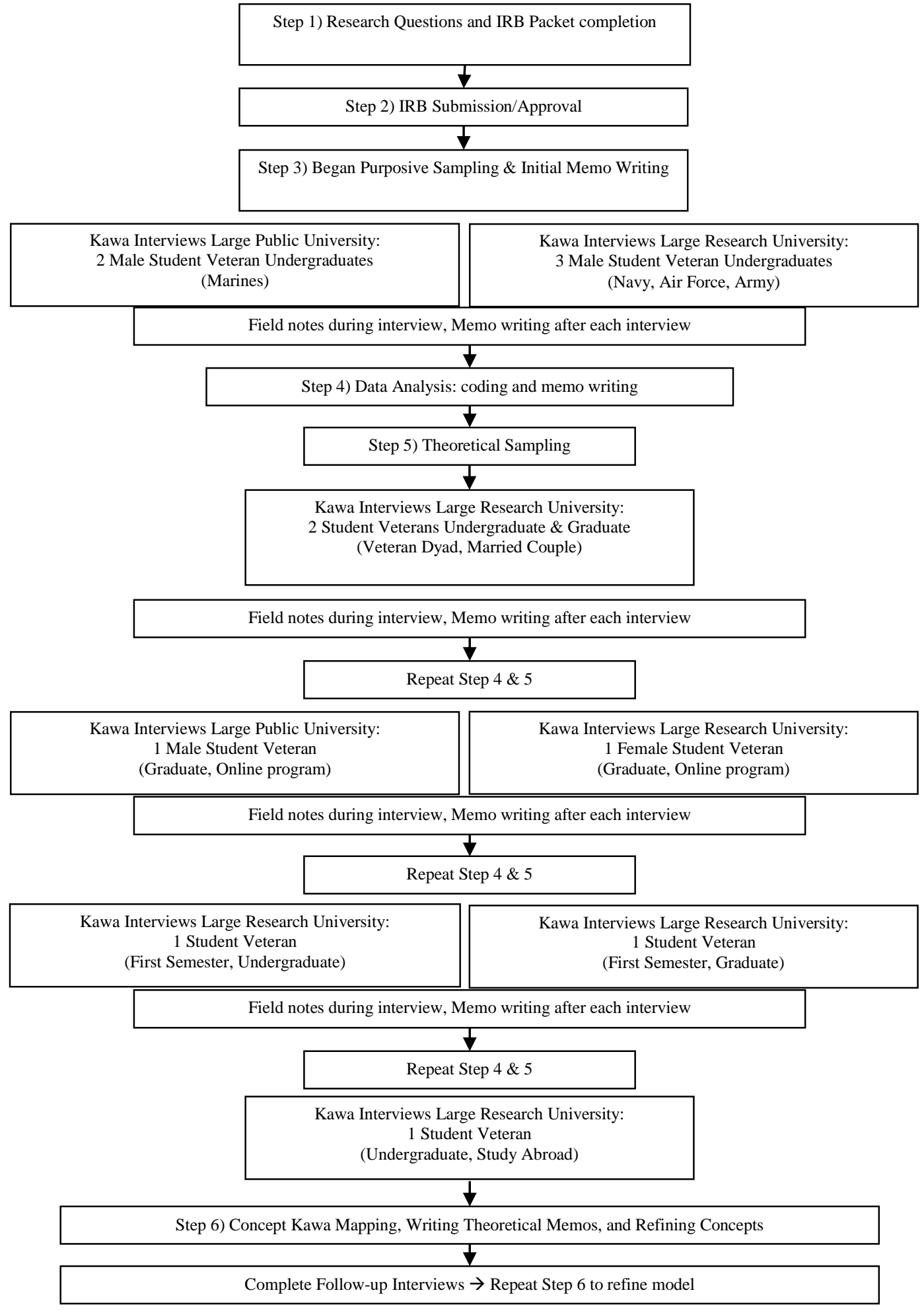




\section{Recruitment}

Eligible participants were initially recruited for inclusion in the qualitative study from responses to study advertisement flyers and emails at two large public universities. The first university was a large public research institution and the second university was a large public regional institution. The principal investigator (PI) recruited participants with posters and/or electronic mail notifications through each university's student Listserv. Participants that had completed to two prior descriptive studies by the PI were contacted by electronic mail to discuss interest in study enrollment. All eligible participants were approached with a description of the objectives and general information of the study by the PI. Eligible participants used this opportunity to query the researcher and received answers to any questions presented. Participants were given the opportunity to freely decline enrollment and participation in the study. In order to maintain anonymity, reduce coercion, and reduce positive response bias, Veteran Resource Center Coordinators/Administrative personnel of each university were blinded (unaware) of which participants had or had not completed the study. No data was collected prior to participant consent.

In addition to the initial purposive method of recruitment discussed above, the PI incorporated the method of snowball sampling common in qualitative research recruitment as a secondary method of recruitment. In this method, the PI also recruited through consenting participants' social groups. For example, at the conclusion of an interview, the PI provided the IRB-approved research study recruitment flyer to the participant and requested that the participant pass along the flyer to other student veterans who have experienced the similar phenomenon of transitioning from combat military 
service to postsecondary education. This method was instrumental in reaching student veterans who did not regularly use each campus Veteran Resource Centers, nor affiliated with the local universities' student veteran associations in an attempt to "blend in" to their surrounding environments (DiRamio et al., 2008).

\section{Participants}

Student veterans currently enrolled, recently graduated (within the past 2 years), or recently dis-enrolled (within the past 2 years) at each university and expressed interest in this grounded theory study were screened for eligibility. Inclusion criteria for the sample included student veterans: 1) between the ages of 20-45, 2) with history of a military deployment, and 3) able to speak, read, write, and understand the English language. Participants were excluded if they were currently serving as an active duty service member, member of the National Guard or Reserves, and/or experiencing an acute psychological crisis requiring medical support. The number of participants needed for qualitative grounded theory studies fluctuates (Creswell, 2013). The fluctuation in participant recruitment is typically attributed to "theoretical saturation" (i.e. the researcher is no longer collecting novel information needed for the robustness of the model), which may be achieved prior to the interview of 20 participants. Therefore, the composition of the participants enrolled consisted of twelve participants as theoretical saturation was achieved. The principal investigator (PI) anticipated participant demographics to at least resemble $85 \%$ male to $15 \%$ female, which is commensurate to a previous pilot study and historical military demographic trends. The PI also recruited participants from all student class levels (i.e. undergraduate, graduate, and clinical professional students). Any participant meeting inclusion criteria was considered for 
enrollment in the study, regardless of gender or ethnic background. Participant recruitment began August, 2015 and ended on 1 March, 2016.

\section{Data Collection}

Data collection included intensive interviews with participants. Charmaz (2014) describes intensive interviews as a, "gently guided, one-sided conversation that explores a person's substantial experience with the research topic" (p. 56). Each initial interview was performed one-on-one and audio-recorded in a secluded area within each university main library and ranged from 34-55 minutes. Interviews consisted of completing a short demographic form modified from a previous study by the PI (see chapter 4) and developing an allegorical diagram utilizing the Kawa (River) metaphor to visualize the constructed life flow for each student veteran. Initially, participants were invited to draw "their river" depicting the experience of transitioning to postsecondary education. The PI

provided general descriptions of the Kawa model and offered suggestions to what each model feature may typically represent. Iwama (2006) defines features of the river metaphor as outlined in Table 5.1: 
Table 5.1. Kawa Model Features and Corresponding Contextual Representations

\begin{tabular}{|l|l|}
\hline \multicolumn{1}{|c|}{ Kawa Model Feature } & \multicolumn{1}{c|}{ Contextual Representation } \\
\hline Water & $\begin{array}{l}\text { Life flow or Life energy of the client } \\
\text { Life circumstances or challenges } \\
\text { experienced by the client }\end{array}$ \\
Driftwood & Assets and liabilities of the client \\
River side-walls and bottom & Social and physical environment of the client \\
Spaces & Areas for occupational therapy to intervene \\
\hline
\end{tabular}

Secondly, the PI queried the participant to confirm their construction of each river attribute and its magnitude or meaning ascribed to their transition experience. Probing questions were used in guiding the intensive interviews and to focus the inquiry on topics areas relevant to the student veterans' life flow when transitioning (see Appendix C). The Kawa metaphor is culturally relevant, completely client-centered, and inductive so the researcher slightly altered intensive interview questions based on responses given by the participants. Questions were asked on the meaning of objects displayed in the participant's graphic and on any actions, processes, and interactions with other people that affected their transition experiences. Participants were instructed to only discuss information they willingly wished to disclose. The PI did not probe into any previous traumatic events, current distressing events, and/or experiences that participants directly or indirectly expressed to the researcher as an uncomfortable topic. Field-notes were written as supplements to the observations of the researchers and annotated participant responses and attributed meanings to their graphic diagrams. Also, all data to include each diagram (Kawa drawing) were de-identified, scanned, and maintained in a secure, encrypted electronic file. 
Once the initial intensive interviews had been recorded, the data were transcribed verbatim and reviewed by the researcher and expert qualitative research study personnel. The participants were contacted by phone or email a second time for follow-up interviews ranging 15-55 minutes, commonly referred to in qualitative research as a member-check. These interviews served to 1) clarify any unclear responses, and/or 2) to ask any follow-up questions that arose in order to reach theoretical saturation.

Participants also used these opportunities to reconcile any questions that had not been answered during the initial interviews. All responses were recorded, transcribed verbatim, and analyzed. Participant data was provided a pseudonym for each transcription that matched their real names on a separate excel spreadsheet. This spreadsheet was kept in a secure area, accessible only to research personnel. This study was approved by the university Institutional Review Board at each institution participants were recruited from and all participants provided initial informed consent and consent for the researcher to contact them at follow-up interviews.

\section{Data Analysis}

Data analysis conforming to CGT methodology departs from previous methods of grounded theory research, (Glaser \& Strauss, 1967; Strauss \& Corbin, 1998) as the focus lies in developing a middle range theory without forcing data into pre-existing categories, while acknowledging the construction of a theory involving the interaction between participants and researchers (Charmaz, 2006, p. 119; Hood, 2007, p. 163). Hood (2007) posits that interpretive forms of grounded theory, such as CGT, may be powerful as long as they utilize the three governing principles of "Grounded Theory" methods: (1) 
theoretical sampling, (2) constant comparison of data to theoretical categories, and (3) focus on development of theory from theoretical saturation versus substantive findings.

\section{Theoretical Sampling}

Theoretical sampling guided the researcher in selecting participants based on the needs of emerging concepts and theory. Theoretical sampling facilitated a deliberate process in which the researchers sought out participants who shared similar responses to research experiences and concepts. Participants were asked "targeted questions" about a particular concept or category of information to verify successive data required for an emergent theory (Morse, 2007, p. 240). Figure 5.1 describes the flow of theoretical sampling procedures used to collect data that enhanced the robustness of the emergent model.

The researchers initially theoretically sampled student veterans from a variety of military service branches (i.e. Marines, Army, Navy \& Air Force) across both institutions. Afterwards, we coded data and wrote memos in order to identify participants that could add heterogeneity to emerging categories. The next two participants theoretically sampled included a veteran dyad or married couple that had both served on active military duty and both transitioned to postsecondary education. Once again, we coded the data and wrote memos that guided the decision to theoretically sample both male and female perspectives in online educational programs. Next, we were able to theoretically sample student veterans entering their first semester of each an undergraduate and graduate program. Following coding and memo writing from these experiences a theoretical model began to emerge, so we decided to theoretically sample another student veteran who had just returned from a year of studying abroad to compare 
the participant's experiences with the emerging model. Once the data fit the model, we used all of the follow-up interviews, member checks to validate the model from the perspective of each participant and to further fill out each theoretical concept for transitioning to postsecondary education. Prior to member checks, the PI sent emails to each participant with an attached document that included, (1) a copy of the emergent model, (2) a description of the model, (3) a list of additional questions aimed at clarifying concepts and enhancing the robustness of each conceptual process.

\section{Constant Comparison}

Constant Comparison analysis in CGT was an inductive process of comparing data with data, data with categories, categories with categories, and categories with concepts, though the focus resided in actions, interactions, and processes (Charmaz, 2006, pp. 178-80). The researcher conducted CGT in a two-step iterative coding process of (1) initial and (2) focused coding in order to render data into theoretical categories. Initial coding consisted of naming each word, line, or segment of data and was defined by Charmaz (2006) in the following ways:

- Word-by-word coding- attending to meaning and flow of each word used by the participant.

- Line-by-line coding- naming each line of written data.

- Incident-to-incident coding- comparing observations of the participant's actions and interactions.

- In vivo coding- coding special terms and meanings within the participants speech. 
Charmaz $(2006,2014)$ subscribes to coding initial and focused codes by using gerunds or action formulations of nouns. The use of gerunds is intended to deliberately situate the data into action statements that fit individual coded experiences together and assist the researcher in exploration of implicit meanings and processes within a research phenomenon (Charmaz, 2006; Charmaz, 2008; Charmaz, 2014; Charmaz, 2015). In initial coding, Kawa interview transcripts were coded line-by-line or with in vivo codes in order to define what was happening in each student veteran's transition and to what extent each action and process meant to veterans. Initial coding involved labeling each segment of data for the initial transcripts reviewed as a gerund thus, generating as many inductive ideas as possible. For instance, concerns were raised by a veteran regarding the use of counseling services in the VA which was initial coded as, 'lacking trust in the VA.' By initially coding each transcribed Kawa interview with gerunds, numerous ideas on the actions and processes influencing student veteran transitions to postsecondary education were produced. In sum, we coded over 640 initial codes from each Kawa interview transcribed ensuring each code was written using gerunds as Charmaz prescribed.

Initial memo writing was performed by the PI to analyze initial codes capturing thoughts on comparisons and connections that elevated the abstract level of the researcher's thoughts on each veteran's transition experience. Charmaz (2015) describes memo writing as "private conversations grounded theorists have with themselves as they take their codes apart and analyze what they might mean" (p. 1617). This process suggested categories for emerging concepts and constantly engaged the researcher in the analysis process of each individual Kawa diagram. Table 5.2 provides an example of 
initial coded memos written to capture the initial thoughts and connections needed to formulate emerging categories.

\begin{tabular}{|ll|}
\hline \multicolumn{2}{|c|}{ Table 5.2 Examples of Initial Code Memos } \\
\hline \multicolumn{1}{|c|}{ Initial Codes } & \multicolumn{1}{c|}{ Memo Text } \\
\hline Needing Understanding & $\begin{array}{l}\text { Needing understanding refers to the unique } \\
\text { realities veterans bring to the postsecondary } \\
\text { education environment and their perceived } \\
\text { exclusivity with their perceived reality. }\end{array}$ \\
& $\begin{array}{l}\text { Having someone is the act of expressing } \\
\text { close attachment to an individual or group of } \\
\text { individuals that bring meaning to veterans' } \\
\text { lives. This category may include family } \\
\text { members, spouses, children, significant } \\
\text { others, and/or close friendships. } \\
\text { Managing Personal Struggles } \\
\text { Managing personal struggles refers to the } \\
\text { complex interplay of veterans' perceived } \\
\text { concerns and the coping behaviors that result } \\
\text { from the concerns. The coping behavior may } \\
\text { be adaptive or maladaptive. } \\
\text { Negotiating social interactions refers to the } \\
\text { conditions in which veterans engage in } \\
\text { meaningful social interactions within the } \\
\text { postsecondary education context. }\end{array}$ \\
\end{tabular}


Focused coding was applied as a directive, selective, and conceptual process to categorize Kawa interview transcribed data "incisively and completely" (Charmaz, 2006, pp. 57-8). Focused coding was used to make comparisons of student veterans' experiences, actions, and interpretations across each interview and observation. The most significant or frequented initial codes from all of the Kawa interview transcripts were compared to develop conceptual categories. After the first three Kawa interviews, we had large amounts of data and numerous initial codes. This included a group of codes that captured how veterans reflected on their previous military experiences in relation to current life circumstances and described internalized conflict for how they defined purpose in life out of the military. Since this process appeared central to veterans' experience with transitioning, and because it was reported often, the PI decided that 'brining stability to military self' should become a focused code. We were able to distill our initial codes using the same process into 15 focused codes as listed in Table 5.3:

\begin{tabular}{|c|c|}
\hline \multicolumn{2}{|c|}{ Table 5.3 Focused Codes and Operational Definitions } \\
\hline Belonging & $\begin{array}{l}\text { Expressing the need to attain/maintain group } \\
\text { membership. }\end{array}$ \\
\hline Bringing stability to military self & $\begin{array}{l}\text { Actions taken to stabilize internalized conflict } \\
\text { resulting from military experiences. }\end{array}$ \\
\hline Connecting to others & $\begin{array}{l}\text { Action of sharing meaning with other people or } \\
\text { groups of people. }\end{array}$ \\
\hline Cultivating student veteran self & $\begin{array}{l}\text { An iterative process for developing identity in the } \\
\text { postsecondary education context. }\end{array}$ \\
\hline Disconnecting with others & $\begin{array}{l}\text { Action of detaching oneself from other individuals } \\
\text { or groups of individuals. Expressed in physical, } \\
\text { social, and emotional aspects. }\end{array}$ \\
\hline $\begin{array}{l}\text { Gaining control in school } \\
\text { environment }\end{array}$ & $\begin{array}{l}\text { Autonomous actions taken by veterans to stabilize } \\
\text { perceived fluctuations in life situations occurring } \\
\text { in the postsecondary education context. }\end{array}$ \\
\hline $\begin{array}{l}\text { Identifying vulnerabilities as a } \\
\text { student }\end{array}$ & $\begin{array}{l}\text { Action of reflecting on current knowledge, skills, } \\
\text { and abilities and perceiving weaknesses in relation } \\
\text { to the demands of school. }\end{array}$ \\
\hline
\end{tabular}




\begin{tabular}{|c|c|}
\hline \multicolumn{2}{|c|}{ Table 5.3 Focused Codes and Operational Definitions (continued) } \\
\hline $\begin{array}{l}\text { Losing control in military } \\
\text { environment }\end{array}$ & $\begin{array}{l}\text { Veteran descriptions of life situations replete of } \\
\text { autonomous actions (implicitly or explicitly } \\
\text { describing a loss of control/power) occurring(ed) } \\
\text { in the military context. }\end{array}$ \\
\hline Making preparations for school & $\begin{array}{l}\text { Actions taken by veterans to prepare for } \\
\text { anticipated fluctuations in life situations following } \\
\text { military service. }\end{array}$ \\
\hline Needing understanding & $\begin{array}{l}\text { A described core belief by veterans that commonly } \\
\text { transition. Participants believed their experiences } \\
\text { are unique to themselves and express a desire for } \\
\text { other individuals to recognize/understand this } \\
\text { perspective. }\end{array}$ \\
\hline $\begin{array}{l}\text { Pinpointing useful resources in } \\
\text { school }\end{array}$ & $\begin{array}{l}\text { A leveraging process of identifying } \\
\text { environmentally supportive resources that veterans } \\
\text { actively use or intend to use in the future to } \\
\text { stabilize perceived fluctuations in life situations } \\
\text { occurring in school. }\end{array}$ \\
\hline $\begin{array}{l}\text { Realizing changes in new } \\
\text { environment }\end{array}$ & $\begin{array}{l}\text { A reflective process of veterans perceiving } \\
\text { fluctuations in life situations occurring in school } \\
\text { that may or may not have been anticipated. }\end{array}$ \\
\hline Reconciling military self & $\begin{array}{l}\text { A transactive process of appraising and stabilizing } \\
\text { self in relation to negative and positive thoughts, } \\
\text { beliefs, emotions, and life circumstances that are } \\
\text { attributed to military identity. }\end{array}$ \\
\hline Regaining control & $\begin{array}{l}\text { The process of applying individual agency on one's } \\
\text { circumstances in school. }\end{array}$ \\
\hline Using military strengths & $\begin{array}{l}\text { The action of applying knowledge, skills and } \\
\text { abilities learned in the military to the task demands } \\
\text { of school. }\end{array}$ \\
\hline
\end{tabular}

Charmaz's coding system relies heavily on memo writing throughout coding, as an abductive process, to make comparisons of data and offer "flexible interpretations of the final concepts constructed in the theory" (Charmaz, 2015). According to Charmaz (2006) abduction, "entails considering all possible theoretical explanations for the data, forming, hypotheses for each possible explanation, checking them empirically by examining data, and pursuing the most plausible explanation" (p. 104). The inherent flexibility differed from Strauss and Corbin's $(1990,1998)$ form of grounded theory 
analysis as these researchers offer a three step process explicated within a conditional/consequential matrix. Strauss and Corbin's third step known as axial coding assists in formulating, "questions about the conditions, actions/interactions, and consequences of categories, thus making links between the ideas being conceptualized from the data" (Mills, Bonner, \& Francis, 2008). Charmaz contends that the use of axial coding may "extend or limit" the researcher's vision during analysis of the data to justify her flexible and/or interpretive process and does not endorse its use (p. 61). Therefore, we applied the use of memo writing to enhance our analysis of the research phenomenon of veterans transitioning to postsecondary education.

CGT uses memo writing to raise conceptual categories to the level of theoretical codes. In this respect, the researcher wrote narrative statements in each memo according to Charmaz's (2006, p. 32) recommended guidelines which are as follows:

- Define the category.

- Explicate the properties of the category.

- Specify the conditions under which the category arises, is maintained, and changes.

- Describe its consequences.

- Show how this category relates to other categories.

Memos of focused codes developed and verified categories of the data as a whole. Memo writing of focused codes assisted the researcher in identifying variance within and between the emergent categories. Table 5.4 provides an example focused coding memo that explicated the emerging properties of veterans stabilizing their life flow in the transition to postsecondary education. By comparing initial codes against initial codes 
and each veteran transition experiences against the collective veteran transition

experiences, we distinguished the category of 'gaining control in the school environment'

from other codes, such as 'managing time' and 'planning for more school'. We were

able to use this process of focused coding to understand the relationships between initial

codes and to further fill out the rest of our 15 focused codes.

Table 5.4 Example of Focused Code Memo

Stabilizing Self + Military + Student Veteran

Two processes that occurred pertained to reconciling the military self and then cultivating the student veteran self. In reconciling the military self, veterans had comments that demonstrated their experiences in the military and how meaningful it was to them to have served their nation. In those experiences, there were positive and negative stories shared that impacted how they perceived themselves. The negative aspects highlighted problems experienced during deployment and in combat as well as injuries sustained on active duty military service. These were all issues that each veteran had to come to some sort of terms in how they perceive themselves and how they wanted to move in a present or future self. The positive aspects were described in terms of military experiences that were perceived as helpful for building traits and discourses of veterans that still persist in their current self. Bringing stability to military 'self' emerged from codes that applied coping strategies or managing personal issues perceived to have incurred on active duty service.

Cultivating student veteran 'self' categorizes codes that demonstrated actions of building a new self, identifying current vulnerabilities or weaknesses in the PSE context, regaining control of problems and using skills and strengths learned in the military to manage personal issues or internal conflicts within the self.

Cultivating is like a performing a personal assessment of potential strengths and weakness given their current life circumstances and then an application of those strengths coupled with learning new skills to apply in order to actualize becoming a civilian adult student. The key here is that it's explained in terms of autonomous actions. 


\section{Theoretical Saturation}

Theoretical saturation implies that categories gathered no longer elicit "new theoretical insights or properties of the theoretical categories" (p. 113). For CGT, the researcher must be open to sorting and integrating their memos into their analysis in order to compare the categorical data collected. The inter-related process of comparing data to theoretical conceptualizations enhances the abstract level of the theoretical categories moving beyond patterns of data while staying connected to the data (p. 113). Theoretical saturation of categories was assured by the researcher asking the following questions:

- Which comparisons do you make between data, within and between categories?

- What sense do you make of these comparisons?

- Where do they lead you?

- How do your comparisons illuminate your theoretical categories?

- In what directions, if any, do they take you?

- What new conceptual relationships, if any, might you see?

In this study we were able to construct two major emerging theoretical categories consisting of 'seeking understanding to shape life flow' and 'gaining stability to shape life flow'. The 15 focused codes derived from the original Kawa interview data was queried using the five previous questions and written into theoretical memos. Table 5.5 provides an example of one theoretical coding memo that was used to develop the major theoretical concept of 'seeking understanding to shape life flow'. In this memo, the questions raised by Charmaz were used to interpret the Kawa diagrams and interview data received and to formulate an emergent experience that reflected the conditions, consequences and interactions of veterans in each postsecondary education context. 
Using constant comparison with the Kawa model, we produced two theoretical codes that captured the social process that veterans went through when faced with a transition from the military and/or novel life challenge(s) associated with the transition. Table 5.6 is an excerpt from Appendix P that summarizes the CGT coding \& analysis process of formulating theoretical codes/categories from participant quotes in their entirety. 


\section{Table 5.5 Example of Theoretical Code Memo}

Seeking Understanding to Shape Life Flow

After analyzing the focused codes and reviewing the emerging categories in relation to each veteran in context, I have identified two major theoretical concepts that occur as veterans transition to postsecondary education. With application of the Kawa model the first process that emerged was 'seeking understanding to shape life flow' and the second process was 'gaining stability to shape life flow'. Both processes were identified to exist on a continuum that fluctuates positively and negatively depending on the actions taken by the veteran or by others interacting with the veteran.

Seeking understanding represented how veterans believed that no one outside of the military understands their experiences or perspective. There was an implicit and explicit description that military service changes an individual and gives them experiences that are novel and unique compared to outsiders or those who have not served. These experiences are assumed to be un-relatable between veterans and family, friends, students, and campus officials, which further intensifies this perspective. This perspective also highlights perceived changes in interpersonal interactions between the collective military sociocultural environment and the individualistically focused civilian educational environment as veterans consider their military experiences to have a different purpose or meaning than what they experience in the educational context. It is reflective and iterative. Veterans either assume no one understands them or discovers through their interactions that they cannot relate to others and feel an intense need to be understood.

Seeking understanding was facilitated by the actions of others to 'reach out and connect' with veterans. All but one of the participants discussed the need to connect with others with an expectation that people within their environment would or should reach out to them in order to enhance social interactions and to enhance the quality of interpersonal relationships. Some veterans stated that they felt lonely, alone, and/or disconnected from the university and friends. There social environment and relationships were difficult to develop, and in the cases of veterans who experienced PTSD, the act of social isolation would occur. From these perspectives, veterans reported, needing a little nudge, or someone to send them personalized email messages in order to connect with others. Some veterans went through their entire educational programs without connecting with others. But, they were also the same participants that reported problems with interpersonal relationships with their spouse or significant others. A couple of veterans stated that the actions of separating themselves from others was a direct reflection of how they were trained to handle combat. As a mission ensues, emotions have to be suspended in order to complete the objectives. This issue of separating or isolating feelings was not addressed as they left the military, therefore many participants were trying to identify how to respond and regain control of their emotions in the civilian and postsecondary education environment.

As veterans connected with others who reached out to them, they were able to share understanding for the various life circumstances they were going through. In some instances, it was connecting with the local veteran support center on campus or in a veteran orientation class. In other instances, it was connecting with family member or community services that offered unconditional support or comradery that was greatly appreciated by the veteran. Four of the participants expressed the importance of church and spiritual practices that supported their understanding of their experiences with the military and at school. For other veterans, seeking understanding was supported by a significant other that would actively reach out in a consistent pattern that forced the veteran to reexamine the importance of the individual reaching out and to personally reflect on the internal struggles they were experiencing. 
Table 5.6 Constructivist Coding Process Summary

\begin{tabular}{|c|c|c|c|}
\hline Raw Data & Initial Coding & Focused Coding & Theoretical Coding \\
\hline $\begin{array}{l}\text { [Evan] One of my first classes here, I was } \\
\text { in this massive auditorium with over five- } \\
\text { hundred seats, and there was only maybe } \\
\text { a hundred people in the class. But the } \\
\text { professor wanted everyone to sit in the } \\
\text { front six rows and fill up every seat } \\
\text { because he did not want to yell at } \\
\text { everyone in the class. That's } \\
\text { understandable, but I can't do that. So, I } \\
\text { had to sit at the eighth or ninth row } \\
\text { because I have to be able to watch } \\
\text { everybody. Cause if somebody goes to do } \\
\text { something they shouldn't I want to be } \\
\text { prepared for it and know how to react. } \\
\text { So, getting professors to understand them } \\
\text { and to not force them to do something } \\
\text { that they would not be okay with. }\end{array}$ & $\begin{array}{l}\quad \text { Accommodating veteran } \\
\text { problems } \\
\text { Being sensitive to outsiders } \\
\text { Explaining veteran problems } \\
\text { Feeling supported by peers } \\
\text { Finding an empathetic } \\
\text { counselor } \\
\text { Finding those who know } \\
\text { combat } \\
\text { Having a hard time with family } \\
\text { Informing others } \\
\text { Lacking empathy from peers } \\
\text { Lacking trust for the VA } \\
\text { Lacking veteran understanding } \\
\text { Periodic occurrence } \\
\text { Receiving specialized training }\end{array}$ & $\begin{array}{l}\text { Needing understanding } \\
\text { (32 Codes) }\end{array}$ & $\begin{array}{l}\text { The process of seeking } \\
\text { understanding to shape } \\
\text { life flow }\end{array}$ \\
\hline $\begin{array}{l}\text { [Shelly] It (the military) revealed things } \\
\text { to me that I didn't know were going on. } \\
\text { Like health-wise. Things that I didn't } \\
\text { know I was composed of... I don't know, } \\
\text { it seems to bring out the best and the } \\
\text { worst of you. If that makes any sense. } \\
\text { The best meaning that, I would say the } \\
\text { best was the resilience. }\end{array}$ & $\begin{array}{l}\text { Feeling supported by friends } \\
\text { Learning positive life lessons } \\
\text { Learning resilience in the } \\
\text { military } \\
\text { Learning to cope with PTSD } \\
\text { Learning to do things on your } \\
\text { own } \\
\text { Learning to self-adapt } \\
\text { Managing self } \\
\text { Managing triggers } \\
\text { Overcoming learning disability }\end{array}$ & $\begin{array}{l}\text { Bringing stability to } \\
\text { military self ( } 22 \text { Codes) }\end{array}$ & $\begin{array}{l}\text { The process of gaining } \\
\text { stability to shape life }\end{array}$ \\
\hline
\end{tabular}




\section{Trustworthiness}

The researcher applied a number of strategies to ensure rigor and enhance the quality of the study. The first measures of rigor consistent with a grounded theory approach included developing an audit trail from the data collected and analyzed. Data that underwent the process of theoretical sampling and constant comparative analysis were documented in qualitative research software known as Nvivo $10^{\mathrm{TM}}$. The Nvivo program served as a repository for all data sources, an organizational tool, and a process for tracking each level of data analysis.

Member-checking was implemented once all interviews had been completed to ensure the phenomenon investigated was well-grounded in the emergent data. During member checking, the researcher contacted participants to comment on the credibility of the findings and interpretations synthesized from the data by the researcher (Creswell, 2013). The member checks facilitated opportunities for participants to judge the accuracy of the interpretive data. Their input was used to establish credibility of the conceptualization and to correct any misinterpretations of conceptual data.

The practice of writing memos and developing field notes guided the researcher in the process of comparing data to data in an iterative process for generating theoretical codes and categories. Memoing consisted of documenting novel ideas and insights following interviews that generated new directions for analyzing qualitative codes and to compare them against emerging categories, whereas field-notes were written in an interview to highlight actions and processes experienced by participants in their context (Charmaz, 2006, p. 22). Together these tools were used to identify and collect different forms of data and to make comparisons within and between each interview. 
Peer debriefings occurred regularly between the researcher and expert qualitative research study personnel. The intentions of the peer debriefings were to "test the insights of the inquirer and expose them to searching new questions" (Guba, 1981). The peer debriefings were used to ensure the PI was limiting bias into the study due to his world view and to ensure data was analyzed an interpreted in concert with the constructivist grounded theory methodology.

The researcher also maintained a reflective journal in order to minimize researcher-participant bias. The primary researcher of this study was an active duty military officer with a shared world view of the participants that brought forth an inherent bias when interpreting data from student veterans. The practice of reflective journaling allowed the researcher to scrutinize his own research experiences, decisions, and interpretations. The practice of reflective journaling was intended to limit researcher bias in a deliberate process for other qualitative researchers to assess the extent the researcher's assumptions influenced his inquiry.

\section{Results}

Table 5.7 summarizes the participant characteristics for this study. Eight of the veterans were undergraduate students and four were graduate students. One veteran in graduate school was a doctoral student. There were nine males $(n=9)$ and three females $(n=3)$ with all but one veteran self-reporting as Caucasian. The mean age of this sample of veterans was 28 years with a standard deviation of 4 years. All but one of the veterans served primarily on active duty. Three veterans were active in the local university Student Veterans Association (SVA) and nine endorsed active engagement with the university VRC. The average self-reported grade point average (GPA) was 3.45. The 
average commute to school was over 8 miles. The average number of schools attended was 1.92 . The average number of deployments was 1.92 and the length of deployments reported was over 13 months as reported by student veterans. Four veterans were married (including dyad) and three veterans had children (including dyad). 
Table 5.7. Participant Characteristics

\begin{tabular}{|c|c|c|c|c|c|c|c|c|}
\hline Pseudonym & Gender & Age & Race & Student Status & Major & $\begin{array}{l}\text { Service } \\
\text { Branch }\end{array}$ & $\begin{array}{c}\text { Military } \\
\text { Component }\end{array}$ & University \\
\hline Zane & M & 25 & Caucasian & Undergraduate & Criminal Justice & Marines & Active Duty & Large Public \\
\hline Evan & M & 25 & Caucasian & Undergraduate & Public Accounting & Marines & Active Duty & Large Public \\
\hline James & M & 28 & Caucasian & Undergraduate & Telecommunications & Navy & Active Duty & $\begin{array}{c}\text { Large } \\
\text { Research }\end{array}$ \\
\hline Jim & M & 22 & Caucasian & Undergraduate & Physics & Air Force & Active Duty & $\begin{array}{c}\text { Large } \\
\text { Research }\end{array}$ \\
\hline Tim & M & 27 & Caucasian & Undergraduate & Economics & Army & $\begin{array}{c}\text { National } \\
\text { Guard }\end{array}$ & $\begin{array}{c}\text { Large } \\
\text { Research }\end{array}$ \\
\hline Brad & M & 31 & Caucasian & Undergraduate & Business Management & Marines & Active Duty & $\begin{array}{c}\text { Large } \\
\text { Research }\end{array}$ \\
\hline Andy & M & 29 & Caucasian & Graduate, Master's & Public Administration & Army & Active Duty & Large Public \\
\hline Kelly & $\mathrm{F}$ & 33 & Caucasian & Graduate, Master's & Business \& Communications & Air Force & Active Duty & $\begin{array}{c}\text { Large } \\
\text { Research }\end{array}$ \\
\hline Shelly & $\mathrm{F}$ & 42 & $\begin{array}{c}\text { African } \\
\text { American }\end{array}$ & Graduate, Doctoral & Educational Leadership & Army & Active Duty & $\begin{array}{c}\text { Large } \\
\text { Research }\end{array}$ \\
\hline Monty & M & 28 & Caucasian & Undergraduate & Electrical Engineering & Army & Active Duty & $\begin{array}{c}\text { Large } \\
\text { Research }\end{array}$ \\
\hline Rachel & $\mathrm{F}$ & 27 & Caucasian & Graduate, Master's & Social Work & Marines & Active Duty & $\begin{array}{c}\text { Large } \\
\text { Research }\end{array}$ \\
\hline Ron & M & 26 & Caucasian & Undergraduate & Chinese Literature & Army & Active Duty & $\begin{array}{c}\text { Large } \\
\text { Research }\end{array}$ \\
\hline & $\begin{array}{r}\text { Mean } \\
(S D)\end{array}$ & $\begin{array}{c}28.58 \\
4.89\end{array}$ & & & & & & \\
\hline
\end{tabular}


Table 5.7. Participant Characteristics (continued)

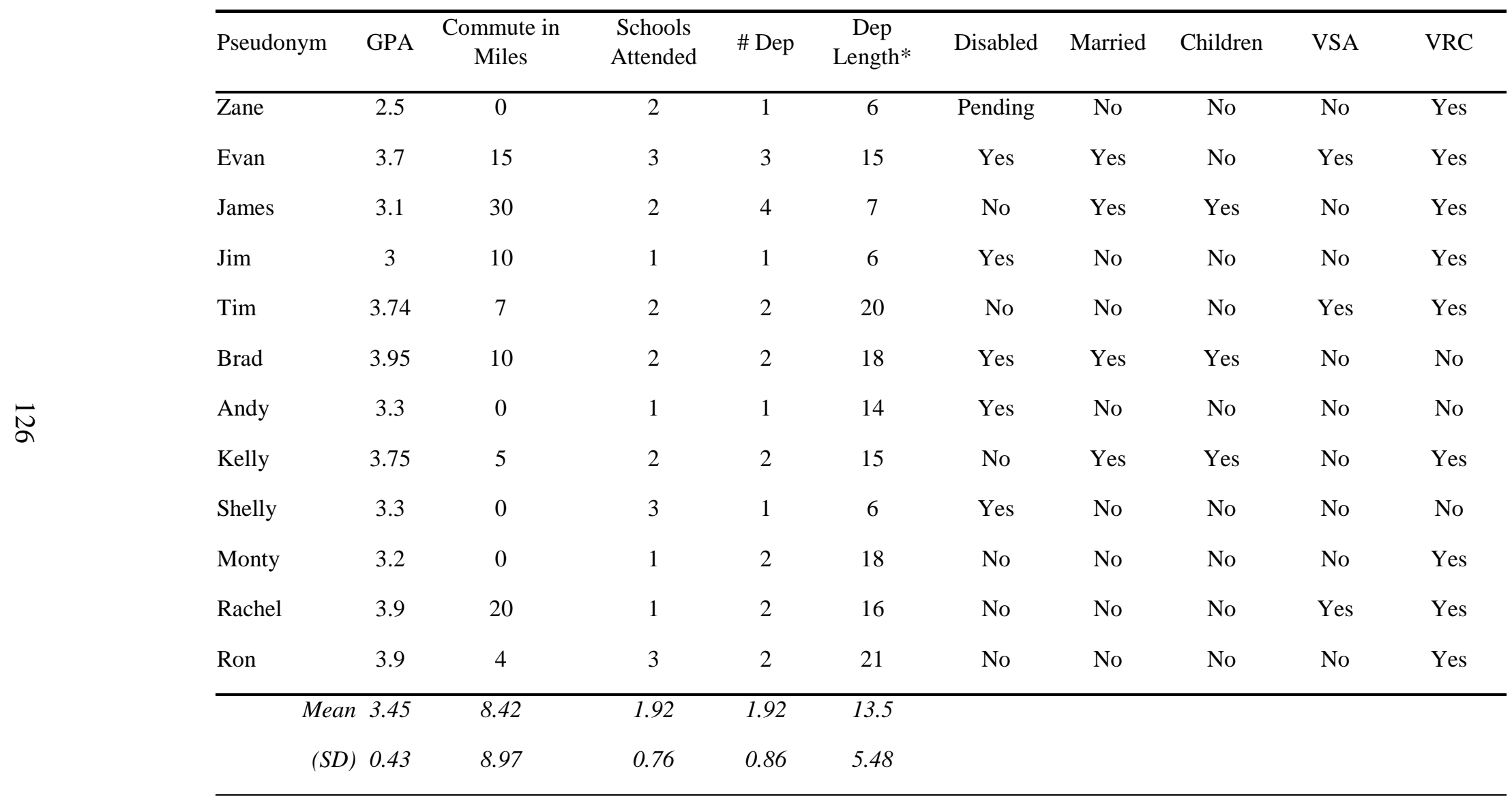

Note. VSA = Affiliation with the University Veteran Student Association; VRC = Endorses use of the University Veteran Resource Center

*Deployment Length reported in total months deployed. 


\section{Situating Veteran Cultural Transitions with the Kawa Model}

Twelve veterans ascribed meaning to narrative responses associated with their Kawa diagrams. The information provided a rich description for Kawa concepts in relation to the transition experiences that affected their life flow. In this respect, the Kawa diagrams shed insights into individual and collective constructions of veteran realities consistent with constructivist grounded theory. Three different environments emerged within the graphic depiction of water, rocks, driftwood, and river sidewalls. The three environments depicted in student veteran experiences included: (1) experiencing military culture, (2) experiencing civilian culture, and (3) experiencing student culture. The PI developed cross-sectional thematic representations to summarize student veteran culturally-related responses. The cross-sectional representations were shared with veteran participants to ensure each diagram reflected their experiences described. Life circumstances reported were depicted as rocks, personal assets and liabilities were depicted as driftwood, and the physical and social environment was depicted as the river sidewalls and river bottom.

\section{Experiencing Military Culture.}

Figure 5.2 provides a cross-sectional view of veterans' life flow summarizing the collective veteran experiences in military culture. Military culture was described as a social environment that provided immense structure for guiding social actions and norms as represented by the side walls and bottom of each veterans' diagrammatic river. The military structure was influential in shaping and in some respects also constraining life flow for veterans. Military structure was attributed toward guiding collective mission accomplishment for all individuals situated in the military context. Individual and 
collective actions experienced by veterans revolved around planning, training, and operations pertaining to a specified mission. In order to accomplish missions and training for missions, veterans had to be unified in sociocultural norms representing a singular belief or professional ethic. The unification of social norms was described in terms of collective military traditions and structure applied from the military environment to support those traditions.

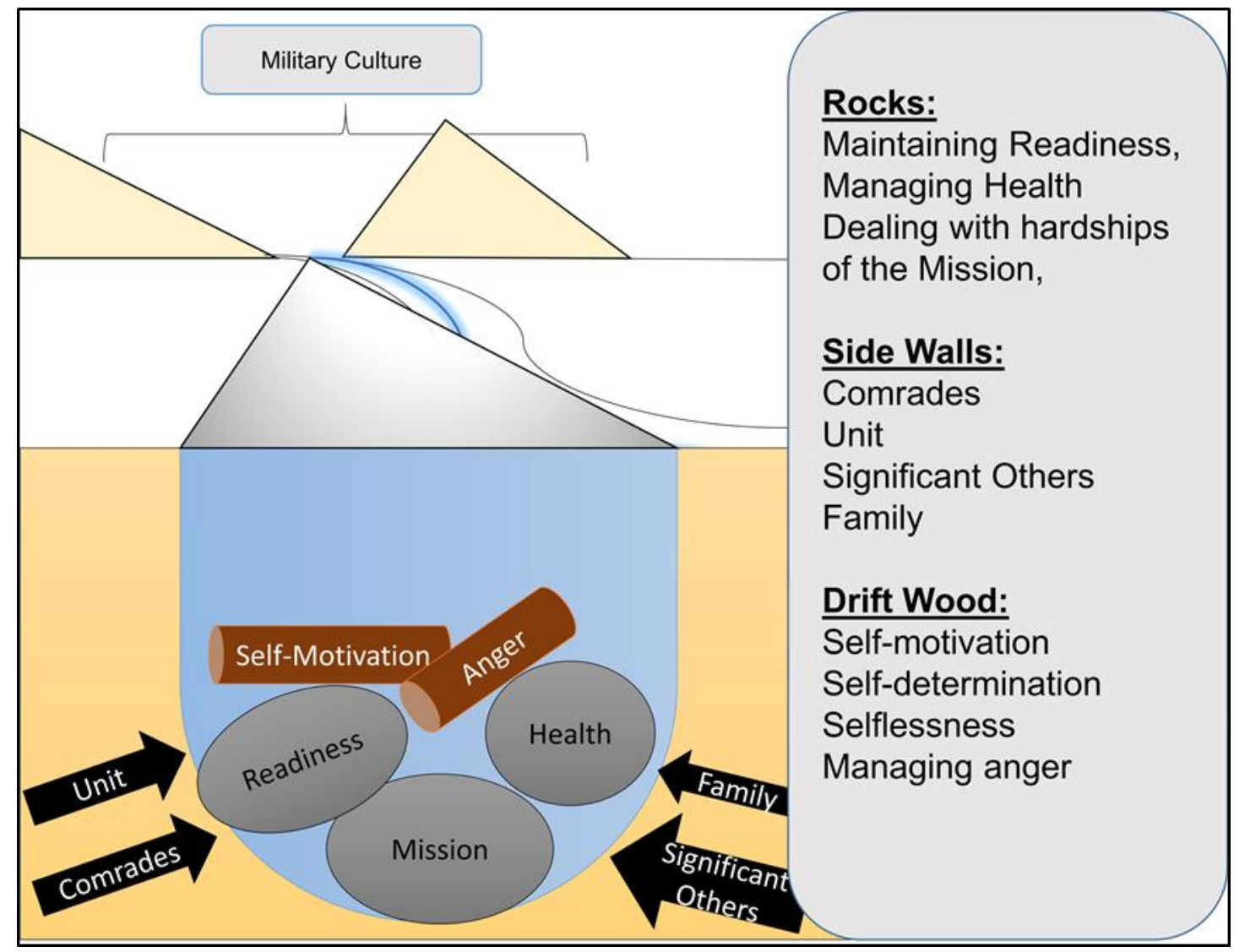

Figure 5.2 Cross-sectional View of Collective Veteran Experiences in Military Culture.

One veteran discussed his interpretation of the military traditions in the following:

[Monty] The military is based on traditions and traditions are rigid. Even with a force that is continuing to evolve the traditions remain rigid.

Another veteran added her perspective on the how the structure of the military guided social attitudes for collective actions in military culture. Her reflection on military 
structure provided a vision of clear decision making that occurred with relative ease in military culture. Her comments also highlighted how expectations were clearly articulated and outlined by military structure in her comments below:

[Rachel] In the military it is very structured and everything is very black and white and clearly laid out for you to get ahead. I mean, to get promoted you know the physical fitness score you need to get or whatever. To get evaluated for promotion, you have to show up on time as a regular thing. There is not calling in sick. They are very structured and expectations are pretty clear and it is pretty easy to navigate.

Other veterans agreed with the structure of the military and discussed how it influenced their world view of military leadership and balance of power within the military hierarchy. For instance, assimilation into the military hierarchy caused one veteran to seek out leaders as mentors who demonstrated the personal characteristics that he perceived was necessary on the battlefield:

[Andy] It was extremely structured. In the beginning, you are trying to absorb everything you can to get integrated into it. As you get more experience you look for people who can guide you and for those who are weak. Now you start pinpointing who is a good leader, who isn't, and who you would want to be around in combat. How they do things, how you would do them differently. Adding to veterans' river side walls and river bottoms were other service members that veterans engaged with. For instance, Andy described the military environment as, "completely inclusive and you are around those people all of the time." Other veterans had discussed how they were "forced" to bond with other service 
members early on in their indoctrination training and subsequent military assignments. However, it was this horizontal bonding with other service members that became necessary for directing focus and purpose on mission accomplishment. These military bonds were described as an essential process for keeping others alive during combat. Monty described how each comrade would rally around each other and the mission to put forth their best collective effort to keep each other alive in combat:

[Monty] Everyone tries to do their best. If you don't know your job, you kill your buddy. That was one thing I heard all the time in the infantry, if you don't do your best, then you just killed your buddy. None of it really seems that serious until the moment it becomes serious... it is true. At some point in time in the military you will see that if you don't do right, you will see how you could kill your buddy.

Monty further described military culture and the how collective purposes of the mission shaped his view of himself and how his comrades had responded to the same collective purposes:

In the military, I never really saw myself. It would always be a reference to 'we' or 'us' and what we did. For instance, we would say, "do you believe what we saw today?" or "you'd never guess what they did to us." I was just there. I never really thought about myself. I didn't want anybody else to get killed or I didn't want anybody else to look bad.

Zane also described how military bonds strengthen for collective purpose in his comments regarding the closeness of military bonding. In his description of military 
bonds, professional attachments resembled the bonds of family as accomplishing missions require interdependence between individual actions and collective purpose. It's like nothing else. Your family, man. Cause like me, we worked out of a patrol base and that's just what it was for us for like three months until they pulled us back to the FOB (forward operating base). It's like, these are the people that we got into fights with and then went out on to patrol with the next day. You got into a fire fight with all of them as well. There's just no way to describe that experience. Even if you don't like them, you still love them. It's like a family member, a cousin. You may not want to talk to them for like a year, but as soon as they call you needing something, you may still have your differences but when it comes to it you set it aside, you just get done what needs to get done. Rocks were commonly described as issues pertaining to health and readiness to complete military missions. The military culture requires a predetermined fitness level that is normalized to the age and gender of the service member. Problems with health affect the readiness of a service member to perform their military mission in a garrison or deployed environment. If health matters become long term problems or chronic issues then the service member is considered incapable of performing work in the military and through a process of medical and administrative evaluation a determination to separate the service member from the military ensues. Shelly described her experiences with health related problems identified in the military:

I thought I was healthy until I found out that I had a dermoid tumor wrapped around my right ovary. It seems like that didn't come about, that pain didn't come about, and it was an excruciating pain where I couldn't even stand up 
straight... And because I got stress fractures in OCS. I found out with the stress fractures and not being used to running, it's easier for you to get fractures. Near the end of my three years in the Army I had what they called idiopathic gastroparesis... It brought me down, emotionally, but at the same time I was already kind of down because it was already at the end of my three years in the Army.

Health problems were experienced by eight of the veterans interviewed in this sample during their military career. The problems varied from mental health disorders to physical health disabilities and often precipitated separation from the military. The health related problems experienced by veterans on active duty serviced created a tumultuous and turbulent set of life circumstances that was perceived as unexpected and immensely stressful. In some instances, the issues experienced with health forced veterans to face a realty without the military under circumstances that were considered a consequence of their own actions. For instance one veteran discussed multiple physical fitness failures for not meeting the military height, weight, and body fat guidelines:

[Jim] I got pretty out of shape and that led to some PT (physical training) failures. And the more and more you keep failing PT, the worse and worse the punishments get. Eventually, I ended up getting demoted because of a third PT failure in four years... I ended-up getting injured and I didn't go through the proper procedures so, they counted it as a failure. And if I didn't fail the first two, which was completely my fault, then I would not have been in that position in any case. 
Jim was forced out of the military due to his inability to pass his physical fitness tests and maintain military height and weight standards. He attributed his "lack of responsibility" as the facilitator for a number of other problems within his life flow as his military separation was unexpected nor had he planned financially for life after the military. Other problems experienced in relation to health were directly attributed to combat exposure such as in Ron's case. Ron described how on an assault mission he "pushed through" his "objective" but the experiences from the moment affected him in ways that he had not considered long after that date:

As far as this date, I don't know if you have ever been to a combat deployment or not, but it's just when my nerves were kind of shot. And I didn't know how much more I could take. Our platoon took a serious hit. A huge operation... ah, sorry... it was a moment for me to push past. And I did it, I finished. I did my job and I went strong and hard all the way to the end. That was definitely the make it or break it kind of point (appears lost for words).

Ron would confess that his experiences guided his decision to "leave the military" but also attributed his "separation with his emotions" and others close to him due to these experiences. He also described how it caused him to make a number of decisions that later affected how much he interacted with others once he left the military.

Personal assets and liabilities were identified as objects resembling driftwood in each veteran's river diagram. In the military, commonly reported assets were described as "showing initiative, motivation or self-determination." Rachel discussed how the culture of the military is focused on promoting initiative in developing leaders that can 
take action during difficult circumstances. Taking initiative serves as directing action to complete missions and advancing toward individual and collective goals.

[Rachel] The military emphasizes in taking initiative. Constant progression and constantly moving forward. You know, the military, number one is mission accomplishment. They are working to complete the mission.

Personal liabilities experienced in military culture focused on managing conflict or anger. The military environment is largely populated with young, male adults that are experiencing their first major career which offers formative, novel experiences. The dominant male culture has been suggested to promote primarily masculine ideals. When veterans get angry, they would use that negative emotion to either focus energy towards a mission or to others. Zane provided an example of how he channeled his inner anger: I can get angry. I don't like to, but making that transition is hard. I was talking to one of my friends about it who was there with me too. Whenever, we had problems we just slugged it out (referring to in the Marines). But, at the end of the day we shook hands and drank a beer.

\section{Experiencing Civilian Culture.}

Figure 5.3 provides a cross-sectional view of veterans' life flow summarizing the collective veteran experiences in civilian culture. Veterans reported being "thrown into the civilian world" and trying to take actions that would help them "stay afloat." The comments of veterans highlighted concerns for unexpected events experienced while dealing with their current life circumstances within a new environment. Problems associated with military service were still present in veterans' lives while constructing a new realty following military service. However, the novelty of being in a new 
environment seemed to be a major challenge in itself. The use of environmental and social supports by veterans were mixed and specific to their individual life circumstances. Veterans in this sample relied, primarily upon family members and/or a significant other as their primary social support (depicted in black arrows). Two veterans described receiving support from the VA for health care during their transition, while others endorsed support from community associations or organizations, such as the local church. The limited use of community organizations and the VA were denoted in white arrows. In all, the transition experiences to civilian culture offered novel stressors compared to each veteran's experiences with military culture. One veteran described his experience with civilian culture in this way:

Well I feel like from the time you first step out of the military, it's just that you have your military life and then all of sudden you are just thrown inside of the civilian world. And trying to stay afloat and figure out how to make that work. There is just so much change that happened all at once. I just felt like everything was moving really fast for me. 
Civilian culture was perceived as more individually focused and less organized which presented different problems for veterans that were in the process of looking for work or

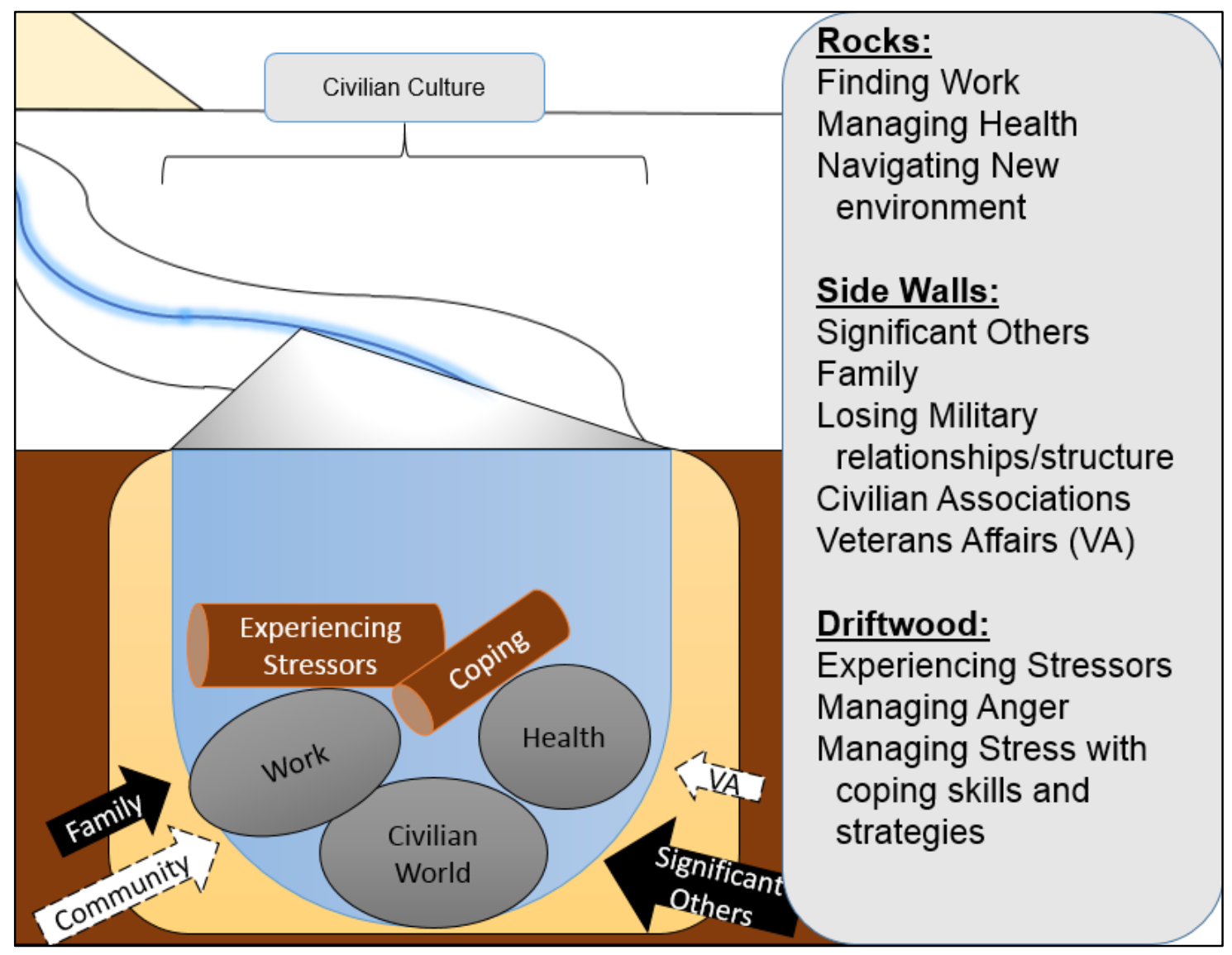

Figure 5.3 Cross-sectional View of Collective Veteran Experiences in Civilian Culture.

applying for university enrollment. Veterans perceived differences in civilian culture as they found new work to support living a civilian life. As veterans engaged with coworkers in a civilian occupation, they also noticed subtle changes in how people in civilian world approach working and the ethical standards applied to work in general.

[Rachel] In civilian culture, it is all subjective. People will have different ideas for what good work ethic is and it seems like everyone has different views. So, when I work at Lowes, their idea of good work ethic is completely different from when I was an air plane mechanic in the military. 
Interestingly, the subjectivity that Rachel described seemingly implied differences in how civilians approach work and how meaning was ascribed to working within the civilian context. Meaning ascribed by civilians in the work setting seemed to be fluid and a stark contrast from military culture. Rachel further elaborated on her experiences with working in the civilian world and how it contrasted with the military in relation to her perception of the goal for work in the civilian world:

[Rachel] Working in the civilian world is like the mission is day in, day out about the customer buying things. It's not as exciting, it's not as appealing.

Rachel, like other veterans continued to discuss working in the civilian world in terms of a different mission which denoted the military cultural influence maintained in her language and communication patterns. The differences she described in civilian work ethic was noticed by other veterans as many identified that the civilian workers "lacked a sense of urgency." Shelly commented that the lack of urgency she witnessed contrasted from how in the military context, even federal employees focused on "expediting work in order to keep missions moving." Rachel also noticed how co-workers treated each other in the civilian world and judged these situations as lacking concern for others:

[Rachel] There is not that much comradery. If I call in sick at work, well ah that sucks for the other dude. But I don't feel as though other people feel bad about it. Veterans noticed a stark difference in being in the civilian world and how relationships and support changed. Networking and participating in social interactions were navigated cautiously. Early concerns for being alone seemed to arise as veterans felt a loss in social support and social structure. One veteran described it this way: 
[Andy] I didn't have to answer for someone else's actions. I went out, I went to my job. I was responsible for me. Then the whole civilian thing started kicking in for me. I hung out with military friends. We were all in the military. When you get out, I moved back to Houston and I was like, I don't have any military friends here.

Interacting with individuals in the civilian environment was a major challenge as the social norms and social cues for communicating were quite different for veterans compared to their experiences in the military. Social norms involving the traditions of military culture called for maintaining "situational awareness" and for consistently acknowledging the military rank of superior military personnel. In the civilian environment, veterans observed how many civilians were not situationally aware of their surroundings:

[Monty] I can't be as serious as I used to be before... It is very frustrating to be around people who have never thought that way before. They just come off being really inconsiderate, I guess. Clueless possibly. It's something that I am working on because you can't live like that, constantly judging other people. It's just really petty. Like there are people talking on their cell phones and walking on the wrong side of the road, I don't know if they are going to hurt someone else. The fact that no one will look at me while I am walking by. They are always looking at their phones. And I don't know why that bothers me so much. It's not like I think I am invisible, but in the Army whenever someone passed you, they would at least look to see what your rank was. 
Socialization in civilian culture was also a challenge for service members as they expressed frustrating interactions involving poor word choices used with civilians. In the military, veterans were used to speaking authoritatively and without concern for emotional responses of those they spoke to. However in the civilian environment, veterans encountered interpersonal conflicts for periodic bouts of colorful language or lack of tactful communication. Andy described one such example:

[Andy] Like, just the way we joked around in the military, we were rude and crude. But you really didn't have to worry about hurting someone's feelings. Immediately, right after leaving, like saying a joke, I was working as a maintenance tech when I first got out and me and a couple of guys there were joking around and I forget what I said but I didn't see it as that bad but apparently it was. So, some people got butt hurt and of course that pissed me off. It ended up being a screaming match. Just little things like that, you know The driftwood of veterans in the civilian environment consisted of coping skills and strategies needed to manage stressors perceived in the civilian environment. The liabilities of veterans consisted of their internalized concerns for their current life circumstances in the civilian context. Whereas, assets consisted of coping skills and strategies used to manage those concerns experienced in the civilian context. Concerns were raised on the health problems that veterans carried with them from the military to the civilian environment. These problems were indicative of mental health problems, physical health problems, and social health related issues. Some assets consisted of activities veterans would do to manage their stress, such as "reading, writing, or seeking support from others." Veterans also focused on developing spiritual aspects of 
themselves in order to find meaning and purpose following a military career. Other veterans described how the pace of daily life changed and how they had to occupy their lives with an increasing number of tasks in order to maintain the intensity of the military culture in the civilian environment. Evan provided one example of increasing his activity level in the civilian environment:

[Evan] My biggest struggle now is overcoming my post-traumatic stress cause I am no longer with those guys that I was working with. My output is not as fast and high intensity as it was. So, trying to adapt to slowing down from what I am used to and all the training I went through every time I deployed about having avenues of approach and having to profile everybody and decide what they might do as actions.

\section{Experiencing Student Culture.}

Figure 5.4 provides a cross-sectional view of veterans' life flow summarizing the collective veteran experiences in student culture. Veterans perceived noticeable differences between themselves and others in student culture due to their age differences and traditional age students. The perceived age differences also included differences in life experience and maturity between veterans and student culture. The rocks that veterans reported in the student environment included concerns about maintaining a living and affording school as well as managing the symptoms of the health-related problems they previously experienced. For veterans transitioning to student culture they 
perceived themselves as being separate from student culture and in some

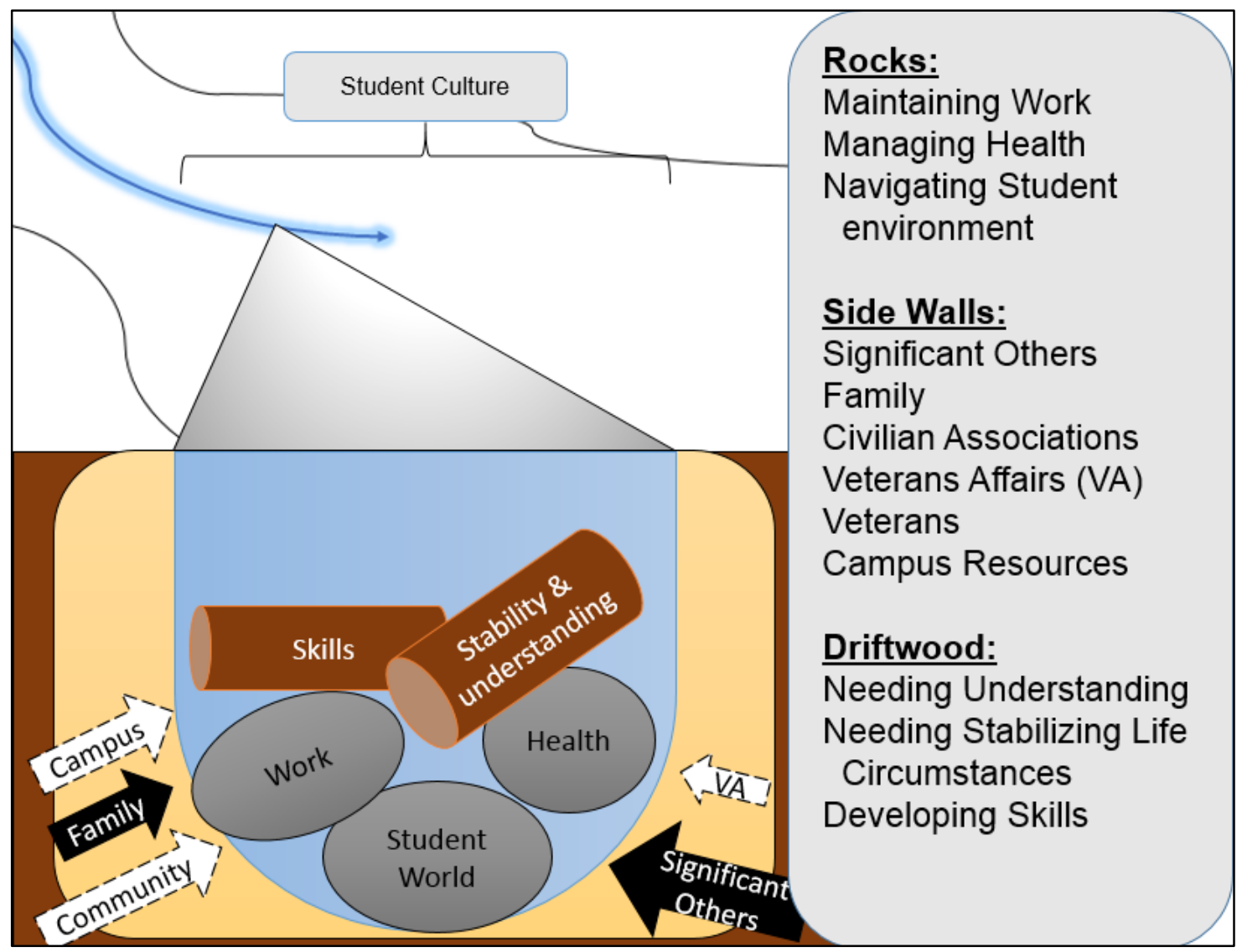

Figure 5.4. Cross-sectional View of Collective Veteran Experiences in Student Culture

cases isolating themselves from socially interactions with other students. One veteran described his experiences of being alone in student culture:

[Monty] In school, I am an island. I really don't socialize with anyone. I just go to class and I come home.

Another veteran described his active choice to isolate himself from student culture due to his perceived differences from other students.

[Andy] I just stayed away from everybody and decided to shut myself down from any stupid stuff with anyone. To this day, in my classes I have not found any people to build friendships with. 
The physical and social support received by veterans in student culture depended on the quality of interactions veterans engaged in. For instance, veterans who sought out the local campus veterans' office received more support over time that would enhance their life circumstances. Similarly, Brad's and Evan's respective river side walls and river bottoms grew through interactions with VA health care, a local church, and/or veteranfocused organizations in the community like the Veterans of Foreign Wars (VFW) organization. As veterans received support from other entities that maintained ties with military culture, life circumstances improved for veterans. One veteran described her experience with other veterans in student culture this way:

[Rachel] I think that it provides you support in that type of soundboard, someone to talk to way. I think it also provides some accountability. I don't know how other students talk to each other, student veterans are certainly more abrasive. So, if I say ah I really don't want to go to class today, they'd probably say, well you are a turd! Like you can't go to class, what's wrong with you? I think that accountability is good. And I think, just genuinely caring, so if you are just stressed out about a paper, you know, you have a goof. They probably will say, hey so what did you end up getting. Did you get to turn it in? I think that the accountability, just the support or soundboard of stuff is what really makes it so important.

Rachel's comments highlighted two issues related to military culture that contrasted with student culture. The first centered on the importance of military values or character attributes developed in the military with the discussion of 'accountability'. The other issue was the difference in communication styles between military and student culture. 
These issues are similar to veterans' experiences in civilian culture, however, they also demonstrated the meaning veterans shared when they socialized with other veterans also engaged in postsecondary education. Rachel also noted that the student environment provided more "structure" and "resources" than the civilian environment and required the use of and development of skills that varied from the typical "9 to 5 job." She further described her interactions in student culture this way:

[Rachel] School forces you to manage assignments, coordinate group work and attend classes while also providing a bunch of resources to help you along the way. It is not like work where when you leave work, you leave work. You don't take it with you.

The assets and liabilities depicted in every veteran's river diagram highlighted how all of the veterans in this study felt a void of shared understanding once situated in student culture. For many this was identified in feelings of loneliness regardless of intentional actions taken to socially withdrawal or to socially interact. The difficulty of being understood was represented in comments of social interactions were understanding was not reciprocated or veterans perceived that other students could not comprehend the meaning of diverse life experiences veterans conveyed within social interactions between other students. Also, being understood was a difficult experience accompanied with veterans describing the need to stabilize their own life flow while engaging in student culture. Stabilizing life flow consisted of the veteran taking individual actions to enhance their life circumstances. Both issues of being understood and stabilizing life flow necessitated a process of connecting to others in order to share meaning with others 
within student culture. Kelly summarized this issue in her river side wall comments regarding social interactions with student culture:

I guess the other side (river side walls) where it is really lonely, it would probably be more like friends than students. I don't really have many friends in school. It was like hard, like I started meeting people in classes but it was really hard for me to connect with them, I should say. And so, I was still around people all of the time. Since I got a communications degree every class project that we did, we would always meet up and do different things but I always felt that I didn't feel really connected with any of them. I started, after a while, in some of the classes they would talk to me as if I was their mom. Started building like a little bit of a relationship, but for me personally, I didn't feel connected with any of them.

\section{Emergent Model for Student Veteran Life Flow in Postsecondary Education}

Examination of veterans' experiences transitioning to postsecondary education using the Kawa model systematically explicated human social experiences situated within three contrasting social environments. Those experiences of veterans within each context were further analyzed using the CGT method to reconstruct those experiences into a coconstructed, emergent theoretical conceptualization for veteran transitions to postsecondary education. CGT analysis of 15 focused codes rendered an emergent model with two theoretical categories (Figure 5.5) conceptualizing the transition experiences in relation to student veteran life flow via a researcher-veteran interaction or process of inquiry. In Figure 5.5, Post 9/11 Veterans described the fluctuations in life flow that 
were attributed to negative and positive life circumstances in each cultural environment in which they were situated within (i.e. military, civilian, \& student).

All veterans perceived their life flow to be clearly 'structured' by the military when situated in military culture. As each veteran moved into a new cultural environment, the need for 'seeking understanding' with others and 'gaining stability' over their individualized life circumstances persisted. The theoretical concepts of seeking understanding and gaining stability gradually improved life flow over time via a process of individual and collective actions and interactions performed between veterans and others situated in their respective social environments. Individual actions veterans performed to enhance life flow consisted of, 'pinpointing useful resources' in civilian and student culture, and 'using individualized skills and strategies' developed in the military to navigate life circumstances outside of the military. As veterans engaged in the postsecondary education, they gradually developed previous skills and 'cultivated the development of new individualized skills', knowledge, and useful strategies that enhanced their life flow.

Veteran transitions to postsecondary education were further explained by the social support structure that was experienced in each environment. The social support structure of the military fostered close attachments to other service members via a process of sharing collective purpose. Being situated outside of military culture created a conundrum for veterans, as the social support was eliminated and interdependence for collective purpose no longer persisted. The lack of shared meaning and purpose created internal conflict veterans where not prepared for. In some ways veterans found themselves searching for others to connect with in civilian and student culture. Veterans 
wanted to share purpose and meaning with others, but with the stipulation that others 'reached out' to them to facilitate shared meaning.

\section{Theoretical Code 1: Seeking Understanding to Shape Life Flow.}

Seeking understanding represented how veterans believed that no one outside of the military understood their experiences or veteran perspective. There was an implicit and explicit description that military service changes an individual and gives them experiences that are novel and unique compared to outsiders or those who have not served. Those experiences were assumed to be un-relatable between veterans and family, friends, students, and campus officials, which further intensified their perspective. Seeking understanding also highlighted perceived changes in interpersonal interactions between the collective military sociocultural environment and the individualisticallyfocused civilian educational environment as veterans considered their military experiences to have a different purpose or meaning than what was experienced in the educational context. Seeking understanding was also described as a reflective and iterative process that changed over time and relationships. Veterans either assumed no one understood them or discovered through their interactions that they could not relate to others which intensified the feelings of needing to be understood.

Needing Understanding. Veterans strongly expressed the need to share understanding of their military experiences with individuals situated in the postsecondary education context. For some veterans, understanding was required for from healthrelated issues sustained on active duty. Veterans requested that university officials such as professors would understand and accommodate the needs of veterans with healthrelated complications. One veteran described how his experiences with PTSD affected 


\section{Emergent Model of Student Veteran Life Flow in Transition to Postsecondary Education}

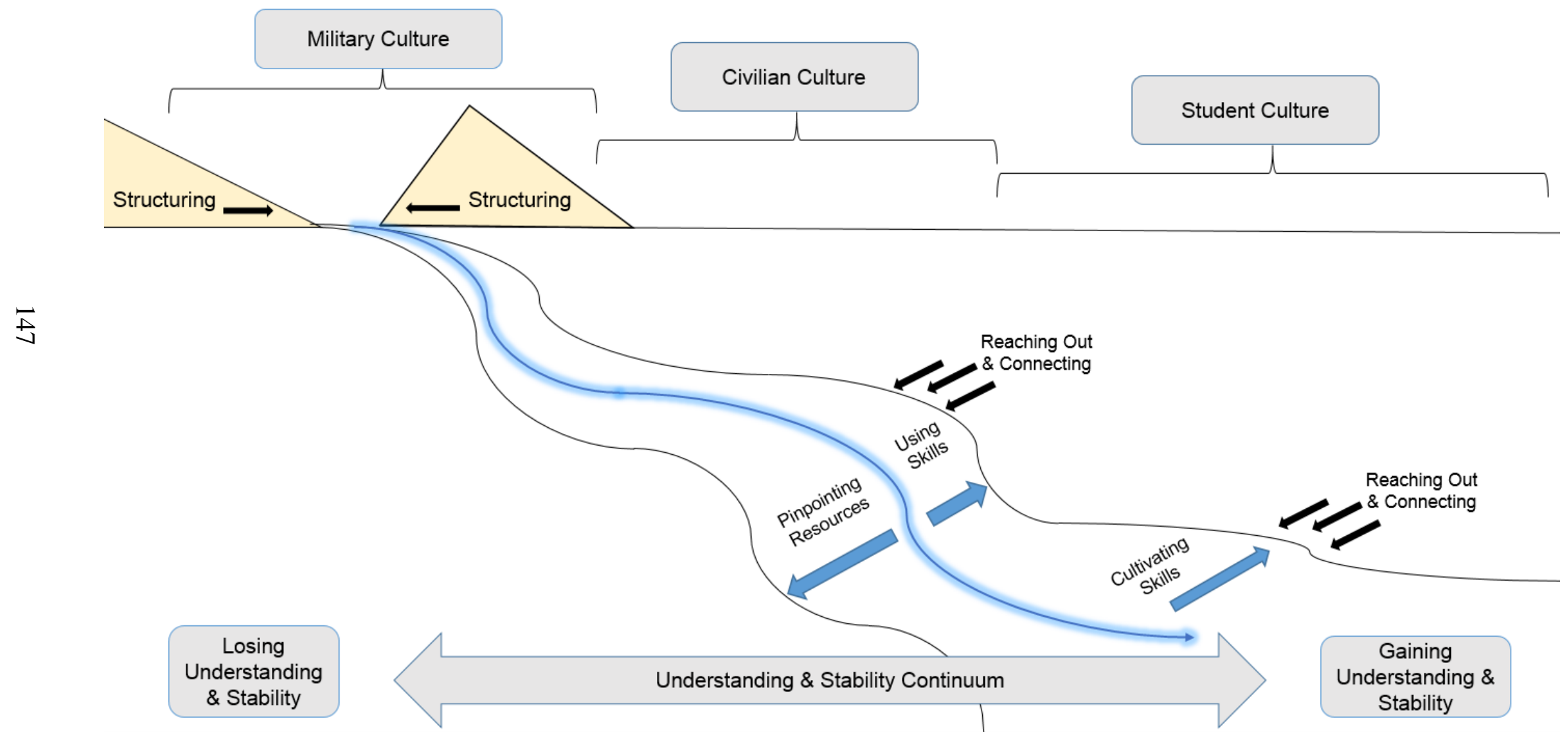

Figure 5.5. Emergent Model of Student Veteran Life Flow in Transition. 
how he approached attendance in class room settings. Due to sensitized concerns for safety and security, Evan would select classroom seating that would accommodate his concerns of being threatened. Being able to observe all individuals in his class from a physical distance offered a risk management strategy for managing anxiety he experienced in the large crowds of university lecture halls. His experience was described in the following way:

[Evan] One of my first classes here, I was in this massive auditorium with over five-hundred seats, and there was only maybe a hundred people in the class. But the professor wanted everyone to sit in the front six rows and fill up every seat because he did not want to yell at everyone in the class. That's understandable, but I can't do that. So, I had to sit at the eighth or ninth row because I have to be able to watch everybody. Cause if somebody goes to do something they shouldn't I want to be prepared for it and know how to react. So, getting professors to understand them and to not force them to do something that they would not be okay with.

In order to share meaning of military experiences veterans sought understanding outside of the school environment. Often, veterans would gravitate towards causes that supported other veterans. For instance, Brad participated with other veteran organizations to share experiences and be understood. His experiences with the local VFW was described below:

[Brad] And another organization that I have gotten involved with is the VFW. I go there once every week or two. And I am on honor guard and do funerals a couple times a month, usually. And that is to be around other veterans that have 
been through similar circumstances and can understand what I went through and can talk to.

Many veterans found understanding in other veterans situated in student culture.

Interacting with other veterans provided an understanding for military culture that was mutually shared and easily interpreted. Through interactions with other veterans, student veterans were able to share the perceived stress and struggles experienced in student culture. One veteran described the significance of sharing her struggles with veterans in the passage below:

[Rachel] Like in the class we were just instantly drawn to each other. Even though I am the female, reservist, air-winger. And they were hardcore infantry dudes. Just the shared struggle of going to class, dealing with eighteen year olds, and homework. Those sort of things helped my transition.

Seeking understanding was a process inclusive of both student and civilian culture. Veterans realized that typical students and civilians did not understand their military experiences either. Veterans described how civilians casually glossed over or minimized the military experiences of veterans as they did not grasp the meaning veterans ascribed to those unique life experiences. One veteran stated the following:

[Kelly] When I first got back from my first deployment and I called up my best friend, and I talked to her about it. And I told her what was going on and like what I did, especially on this first deployment, when the war first started. She was like, oh that's cool, well do you want to go to the mall (Kelly laughs)? Like she really couldn't even relate to anything that I said. And I was like, okay. So, it just turned back to like, superficial, regular world stuff. 
Some veterans acknowledged that others within the student environment made attempts to understand them. However, these attempts were sympathetic at best, and did not have the resounding impact veterans were hoping for that empathy brings. Zane provided an instance of these experiences below:

[Zane] They don't really understand the transition because they are not going through it themselves. And then they may know somebody who is going through it, but until you experience it first hand, they don't actually know it. They are still willing to help you out and let you know they are there.

For other veterans, sharing understanding meant being vulnerable and open to the interpretations of others. Veterans frequently demonstrated reluctance to being vulnerable to students that they could not share meaning with. Understanding was best facilitated with other veterans that had experienced similar situations in combat and the military. One veterans provided the following insights into this topic:

[Andy] Honestly, I am not going to talk to someone that hasn't experienced combat about combat. So, I mean the only real way to do that is to get some sort of peer support group where you know that the person you are talking has somewhat been there, done that. As opposed to trying to create something else that is open to everybody and I am going to sit down and talk to civilians who are going to look at me like I am a horrible person for the things that I have done. Beyond that, I don't think that on a social level the university can do much more. Because they have so many other responsibilities to all the other students.

Reaching Out and Connecting. Seeking understanding was facilitated by the actions of others to 'reach out and connect' with veterans. All but one of the participants 
discussed the need to connect with others with an expectation that people within their environment would or should reach out to them in order to enhance social interactions and to enhance the quality of interpersonal relationships. Some veterans stated that they felt lonely, alone, and/or disconnected from the university and friends. Relationships were difficult to develop, and in the cases of veterans who experienced PTSD, the act of social isolation would occur. From these perspectives, veterans provided examples of, needing a little nudge, or someone to send them personalized email messages in order to connect with others.

A few veterans went through their entire educational programs without connecting with others. Often, these were the same veterans that reported problems with interpersonal relationships with a spouse or significant other and endorsed PTSD symptoms. A couple of veterans stated that the actions of separating themselves from others was a direct reflection of how they were trained to handle combat. Veterans rationalized this response as a product of preparing for military missions. Emotions were reportedly suspended in order to complete the objectives. However, this issue of separating or isolating emotions was not directly addressed as they left the military, therefore many participants were trying to identify how to respond and regain control of their emotions in the civilian and postsecondary education environment. Initially, connecting with others began with socially interacting with family members that veterans had been isolated from during their military career. One veteran discussed his renewed connection with family below:

[Andy] my mom and my dad. Even though they are not together, they would always ask me how school was going. You know, growing up we didn't get shown 
a lot of affection so, it was good because they were taking an interest. And they were like wow, I can't believe you got a 4.0 and you are taking seven classes a semester plus you are working.

Another veteran provided insights into how family would reach out to him once he started school. Family members reaching out enhanced Zane's personal well-being as he discussed the positive emotions following family members reaching out to him:

[Zane] I have a seven year old nephew that loves me to death. I am actually taking him out next weekend for the first time. They [his siblings] always just call and check on me to see how my day is going. Just doing little things that make me happy. They are always there for me, though.

One veteran talked about re-connecting with a step-dad that reached out to him and the impact of their relationship early on:

[Monty] I got my, I call him my old man. He was like a step-father at one point in time. But I kind of hung around him because he taught me how to be a man. He influenced me a lot. He was around for my teenage years or my formative years. He used to be in the Army and he is constantly calling and checking up on me, cause of different stuff.

For a veteran dyad that was interviewed, understanding for problems experienced in student culture was co-shared and enhanced the quality of interpersonal communication. The married veteran couple was able to support one another through their shared understanding of student culture and educational task demands. The following insights were provided: 
[Kelly] he would ask me a lot of questions, so what do you think about this or what do you think about that? I think that is what made me really love going to school. Cause I really enjoyed learning all these new things. And I enjoyed that I could see the world differently. And stretch myself, intellectually. He just supported me like if I had homework, he would let me do homework or he would take our daughter to get ice cream. If, I was in a panic because I had two assignments and an exam and this all going down or if I was in a bad mood, he knew I was stressed. He would understand what I was going through. He wouldn't like try to argue with me or fight with me. He just knew that I was stressed out.

For another veteran sharing understanding for his military experiences with a significant other caused him to re-evaluate the importance of that person in his life. Through the actions of Ron's significant other consistently reaching out to him, he experienced a revelation and formulated a re-appreciation for what his significant other meant to him. Ron stated:

[Ron] Through a sequence of events, being involved with other students, I began to realize just how much in common I didn't have with my fellow classmates. I began to realize that there were things that I kind of needed to wake up to. Kind of like re-circle around to a re-appreciation for who she [his significant other] was and what she meant to me. And the origin of this soul to me.

One campus resource that was attributed to connecting veterans with others that could empathize with their transition experiences was the campus veteran office or veteran 
resource center. One veteran discussed the importance of his interactions with the local VRC in the following way:

[Tim] I think the VRC connected me. I think is what it was. I think the VRC as far as, I guess it would be like the side walls because it kind of connected me, I wouldn't say to the ocean so to speak, that would be like getting my degree. But it kind of connected me to campus to campus life. And finding like a group, (meaning campus groups), which I think is like very important. And I guess that the bottom, I guess the thing that was underneath everything, I would say was the VRC coordinator.

Being disconnected from others in the student environment was a common occurrence experienced by veterans. Some veterans attributed disconnection to their PTSD. One example was provided by Brad which shed insights into how his interpersonal relationships were affected:

[Brad] Just with my wife. She felt like I was, I say she... I was unemotional around the house. Distant in a lot of ways. And that caused a lot of frustration with her. Some marital issues, we definitely worked through that with time. It was definitely hard for both of us at the time, at home. That extra stress did make it harder and it did kind of carry into your school work.

Other veterans discussed the importance of others reaching out to them in order to begin a dialogue that could address negative life circumstances experienced. One veteran provided the following insights:

[Evan] A veteran won't come out and say, “here is some of my problems and put them on the table." But, if somebody probes first to see if you are having any 
problems, there may be a chance that he says, well yeah there really is this

problem. And once you help them with one, it could maybe turn him away from a potentially bad situation.

As veterans connected with others who reached out to them, they were able to share understanding for the various life circumstances they experienced. In some instances, it was connecting with the local veteran support center on campus or in a veteran orientation class. In other instances, it was connecting with family members or community services that offered unconditional support or comradery that was greatly appreciated by the veteran. Four of the participants expressed the importance of church and spiritual practices that supported their understanding of their experiences with the military and at school. For other veterans, seeking understanding was supported by a significant other that would actively reach out in a consistent pattern that forced the veteran to reexamine the importance of the individual reaching out and to personally reflect on the internal struggles they were experiencing.

\section{Theoretical Code 2: Gaining Stability to Shape Life Flow.}

Gaining stability to shape life flow referred to a process of veterans experiencing changes associated within each context following military service and applying autonomous actions to support their individualized transition. Veterans performed individual actions to regain control over their life circumstances in student culture. Gaining stability was an active process precipitated by individualized decision making of veterans. Leaving the military environment was described as an experience of losing control and/or confronting unanticipated events that veterans initially unaccounted for. 
As veterans began the separation from the military, veterans made initial preparations to leave the military and to develop viable opportunities to sustain civilian and student living. In a making preparations period, all of the veterans expressed that the transition assistance program (TAP) provided by the military was useful. They saw the necessity in using the TAPs program, however, all veterans queried felt as though TAPs did not accommodate or address their individualized needs. As the TAPs program varied across military bases, the content of transition education and training also varied. Veterans identified the lack of insights provided for pursuing postsecondary education. Veterans acknowledged that the only recommendations for postsecondary education offered in TAPS were to seek out VA educational counselors once they transitioned to a community of choice outside of the military. These experiences suggested a need to reexamine the function of the TAPs program and to what content is discussed regarding veteran transitions to postsecondary education.

After leaving the military, veterans formulated inferences or expectations for the environment they were entering and came to realizations that challenged previous expectations. Veterans utilized the skills developed in the military to initially gain stability in student culture. However, the most determinant action veterans took to support their life flow involved actions in 'pinpointing useful resources' needed to enhance life flow. As veterans pinpointed useful resources in the postsecondary education environment, they were able to 'cultivate their current skills and develop new skills' learned in school.

Using Personal Skills. Using personal skills highlighted autonomous actions taken by veterans to stabilize perceived fluctuations in life situations occurring in the 
postsecondary education context. The action of applying knowledge, skills, abilities, and strategies learned in the military enhanced life circumstances and task demands of the postsecondary education. Personal skills included both specific and broadly applied individualized abilities to resolve negative life circumstances. For instance, coping skills and strategies for managing stressors in student culture were applied by veterans. Other skills such as establishing daily routines or managing structured daily schedules were useful for managing time and providing focus for completing assignments in the postsecondary education environment. One veteran reflected on how he learned to approach problem solving independently for novel situations through his military training:

[Andy] I mean you had the team work, but honestly in order for you to come up with new skills and overcome a new challenge, you have to sit down and do it yourself whether it was learn how to break down a 2-40 bravo blindfolded like your team leader wanted you to do or understanding a new concept in my weapons of mass destruction class. That was definitely something the military taught you. It was like, hey, you are going to have help, but what happens if you don't. You need to be able to do this on your own. If you can't, you need to be able to ask somebody and figure it out so you know.

Another veteran discussed how he stabilized his PTSD symptoms by gradually exposing himself to environments that provided a level of anxiety that was manageable or within his perceived tolerance level. By sensitizing his anxiety responses to smaller civilian crowds, Evan was able to better adapt to student cultural environments. His comments were provided: 
[Evan] So, I just kind of self-adapt. If I see there is an issue, I kind of remove myself from the issue. I go to Walmart or public places late at night when there aren't that many people. If, I feel an incident coming on, as a real, real high anxious incident, I can usually detect it. And remove myself and just, step back. Think about what is going on. Go through my threat analysis and different things. I am usually just able to get over it and move on.

For Brad, gaining stability over his PTSD symptoms involved seeking participation on a church sanctioned mission trip to Africa. Having prolonged exposure to individuals of the Muslim faith changed his perception of those who practiced the faith of his prior enemies. The action of mission engagement facilitated a process of re-evaluation and closure for his previous negative experiences affording Brad stability over his previous issues. Brad's comments were as follows:

[Brad] I'll say one of the biggest things that helped me keep moving was I actually went on a mission trip to Africa. I didn't know it at the time but the tribe that we went to go and speak to was over $95 \%$ Muslim. And at that point in time, I actually still hated Muslims after going on two deployments and trying to kill them and them trying to kill me. It was something that I didn't want to do (going to Africa). But I went and I just thought going there that they are people too. They need love and they need compassion. I've seen lives that were changed... at that point in time, I no longer looked at them as the enemy. I think that just getting past that, kind of helped getting over my PTSD overall, because I no longer had an enemy that I wanted to fight. 
Shelly attributed listening to her doctor as a skill for gaining stability over her health complications. As she struggled with a number of issues sustained on active duty that ranged from an ovary tumor to major depression, listening to her doctor guided her decision to consistently take her medications. Even though her medications had a number of side effects, she noticed the benefit of being balanced versus being unstable. She stated it in the following passage:

[Shelly] I am a little bit more at ease with that side (depressed side) of myself, but I know that it has helped out a lot in doing what the physician has said to do. Take the medication to keep things balanced. And it took a while for things to kick in for me because I didn't want to take my medication. And then I felt like I was doing okay without it until I realized I wasn't.

Other veterans discussed skills that the military culture taught them that they consistently applied in school. For Ron, personal accountability resembled a trait that he maintained from his military experiences. Ron discussed how using personal accountability assisted in gaining stability over his particular life circumstances:

[Ron] I will start with the most important lesson I learned in the military. Personal accountability for your own faults and then I would go into a philosophical level. Struggling with the concept of balance. I always felt like I have existed in extremes since I was nineteen. I joined the military, then I deployed to Iraq, then I come home and I decide to dump the military, and then I go to Afghanistan, and then I plunge out head long on this super serious life long relationship. And then in the extreme reaction to that I feel like I need to center 
myself again and disappear into the bowels of China. So, for me it's been trying to find balance and trying not to wade into these extremities.

Pinpointing Useful Resources. Pinpointing useful resources represented a leveraging process for identification of environmentally supportive resources that veterans actively used or intended to use in the future. These resources were perceived to stabilize fluctuations in life situations occurring in the postsecondary education context. Useful resources consisted of campus policies, key personnel, and/or specific services provided to veterans and to students in general. Veterans would prioritize resources on their current need and interests, therefore selecting the best fit for their current problems or life circumstances. Also, resources that were considered of little value were identified as resources to avoid or disregard. Rachel pinpointed the importance of administrative policies that assisted in her academic progress during a deployment in the reserves following active duty. She also acknowledged the role of the VRC director in developing the policy to support academic progress of deployed military student in the below passage:

[Rachel] there is a lot of like administrative stuff. So, like when I did get deployed. Like they put me on a hold or something. I didn't have to reapply when I came back the next year. And I don't think the university had a policy before that. But, I know the VRC director is the guy who worked on that stuff. They've got like a military leave policy now.

Ron pinpointed key personnel within the university student affairs center that supported his academic progress in administrative matters. His comments highlighted the level of 
satisfaction he felt with the services provided by key personnel who were familiar with veteran administrative processes:

[Ron] When I first came to school, I got a certain email with all of the directions. I love the fact that all I had to do was apply for the GI Bill and email them. I just had to talk to a representative and I had never seen here face-to-face, but she has always executed my paperwork. Always taken care of things. For example, like coming back from China, I didn't use my GI Bill last semester, I had to pay for myself. And she was like oh no, all you have to do is fill out a little extra paperwork.

Tim pinpointed the campus veteran service director as a substantial asset in developing his resume. Other veterans acknowledged the conflicting guidance received for resume development on campus, however, Tim's experience with the VRC director were novel as the guidance he received was tailored for veterans. He was able to transfer his military experiences into a cohesive format understood in civilian vernacular. His comments were as follows:

[Tim] I met [the VRC director] and asked him to look at my resume and he did, cleaning it up very well. Gave me some great pointers on how to civilianize my resume. Rather than say, use jargon that is tailored to civilians than military personnel. I did research on my own. I figured out that I wanted to major in economics and that would point me in the best position for a good job.

Brad recognized the need for resume writing, but he also offered a suggestion for a service in addition to or even more important than resume writing. Brad's primary 
concern was for gaining employment to support his family and stabilize his life flow. His concerns were noted below:

[Brad] Just something to help find a job. And I know other universities will offer resume building and things like that, but it doesn't really help you find a job.

Obviously, they can't just hand you a job but, anything they can to help narrow down places you can apply.

Cultivating Personal Skills. Cultivating personal skills represented the growth of skills that veterans perceived as having once situated in student culture and gradually building on those skills as they learned new skills while in school. In this respect, veterans had identified their personal vulnerabilities or weaknesses in student culture and sought to gain stability by enhancing their areas of perceived strength. The key to cultivating personal skills focused on explicit autonomous actions taken by veterans to actualize a holistic view of self. Cultivating skills embodied internalized and externalized skills and traits. For example, Zane discussed how he cultivated his ability to persevere during difficult life circumstances. Persevering was made possible by actively focusing on striving to reach his goals and overcoming his shoulder injuries: [Zane] I want to strive, it sounds cliché, but to be the best I can be. I had surgery on my shoulder back in the summer, so I wasn't in the gym until August or September and I could just do nothing. I got cleared to do more and I just feel so much better. It's because I am able to hit the gym again. But it is just such a relief. Like I had my goals set aside and that I could not reach them, means I had to continue to develop myself. I had to get stronger. I had to keep on. 
Tim discussed his cultivation in the personal trait of resiliency. Early on in his interview, Tim acknowledged that he did not have the resiliency needed to overcome a bad academic semester. However, once he embraced the struggles he had experienced in education, he felt that he had cultivated his resilient self.

[Tim] I have actually built resiliency through my college degree, finally. I think that the best positive changes, I have learned that the biggest positive change is that I have embraced struggle, rather than have avoided it. At this point now. I see the value. I think this is the first time in my life where I have seen the value of struggle. And I guess that comes from like my college courses and so on and so forth. And in a broader sense, my life experiences. But in a more narrow sense and in more tangible experiences some of my college courses were like really difficult like my stats exam or my stats courses. And difficult math courses. Because when I really struggled to get something, when I had that a-ha moment, I think that was one of my biggest changes as far as my mentality. I think if I would have this type of mentality prior to coming to school, I would have been fine.

Shelly discussed the significance of cultivating computer skills during graduate school. To her, developing computer skills in word processing, managing excel spreadsheets, and statistical software enhanced self-efficacy and self-esteem in completing school assignments and independent research. Shelly's comments were as follows:

[Shelly] I am thinking about the computer skills for some reason. It was very helpful for me cause my computer skills were really bad before grad-school. And I am not saying that I am some type of computer geek or something (laughs). I'm not, but I do understand Excel a lot better than before. SPSS, like I am sure it has 
already changed since I have been out of the graduate program (referring to master's degree), but at least I had an understanding for it. And it wouldn't be as hard to catch up with the newer program... It was just so empowering!

Kelly's experiences in student culture gave her a greater appreciation for international students. She felt that she was able to cultivate cultural sensitivity to others. In doing so, she was able to examine and stabilize the biases she internalized towards others. [Kelly] I feel like I am more aware, internationally, other people of other cultures. Just being at the university, I don't know how many countries of students and nationalities that we have here. I got to meet a lot of international students and I felt that was a big deal. I mean, yes when I was in Las Vegas, I got to meet a lot of people from the United States. So, you are like thrown in from different people from like New Jersey, Texas, and North Dakota. So, you got an understanding of the different demographics within the United States. But I feel like sometimes when you go to college, if you look for them and I feel like I did, I got a chance to meet people. Like I got a chance to meet a people from Nepal here and from India, China. So, that was really cool being more sensitive of people from other cultures and learn about other cultures.

\section{Discussion}

In this study, student veteran transition experiences were examined with the Kawa model as a novel approach to understanding the respective cultural environments veterans transitioned in, between, and to. Culture was broadly defined as, "the shared experiences and common spheres of meaning" to include "the collective social processes by which distinctions, meanings, categorizations of objects and phenomena are created and 
maintained" (Iwama, 2006, p.8). The Kawa construct of life flow clearly explained veterans transitioning in this study, as a theoretical conceptualization emerged of student veterans seeking understanding and gaining stability with passage from military culture, to civilian and student culture. Veterans shared understanding for their life circumstances experienced in each cultural environment by socially interacting with others perceived to be reaching out and connecting with them. Veterans also performed autonomous or individualized actions of gaining stability by using their skills, pinpointing useful resources, and cultivating the growth and expression of personal skills. These two theoretical processes collectively, enhanced each veteran's transition to postsecondary education and promoted harmony within veteran life flow over time.

In this study, seeking understanding for life circumstances involving health and well-being were particularly salient as eight of the veterans reported mental or physical health problems related to military experiences. Though we did not set out to theoretically sample veterans with health-related concerns, the high frequency of veterans self-reported health concerns was uniquely informative and must be accounted for in future studies. Veterans voiced a lack of understanding by their contemporaries due to performing school work and perceiving problems with health; in particular mental health problems. Historically high rates of substance abuse and mental health problems had been observed in large epidemiological studies of military populations compared to civilian populations (Substance Abuse and Mental Health Services Association [SAMSHA], 2014) and assessed in student veteran populations (Ellison et al,, 2012). However, in a recent study by Eakman, Schelly, and Henry (2016) risk and vulnerability factors for PTSD, depression, and somatic symptoms have been demonstrated in student 
veterans compared to propensity score matched non-veterans. This study adds explanatory reasoning for why student veterans feel misunderstood by other individuals situated in student culture and accounts for mental health related conditions sustained in military culture as prognostic indicators affecting life flow of veterans.

Seeking understanding was a process that progressed relative to the quality of social interactions and relationships formed. In this study, veterans reported challenges relating to other students and felt unconnected to campus which is commonly identified in the literature (Ackerman et al., 2009; DiRamio et al., 2008; Elliott, Gonzalez, \& Larsen, 2011; Jones, 2013; Olsen, Badger, \& McCuddy, 2014; Rumann \& Hamrick, 2010; Tommar \& Stoffel, 2014). Our results regarding the quality of social interactions and relating to student culture are most consistent with another study that examined the transition of veterans to civilian culture (Koenig et al., 2014), as difficulty relating could be attributed to differences in life experiences and between the lexicons of military culture and traditional students. However, we have added the inclination of veterans to expect others situated in student culture to reach out to them.

The action of others reaching out to veterans assisted in being connected to campus and facilitated meaning and purpose. For veterans that were not connected to others, purpose and meaning was sought via organizations outside of student culture. When veterans did not connect with a community-based organization, the need for belonging with comrades who could share meaning in collective purpose was reported and lasted indefinitely. This finding was significant as veterans expressed how assimilation into military culture induced collective purpose and meaning for mission and goal achievement. Regaining understanding for individualized purpose and meaning in 
student culture by others reaching out was attributed to enhancing life flow. In essence, when others reached out to veterans, veterans were more inclined to use campus resources, supports, and begin socially participating within student culture.

In this study, veterans initially struggled with transitioning from a collectively oriented culture to cultural environments that were individually-focused. To manage internalized changes to personal identity veterans would use their personal skills and strategies to cope with perceived stress, anxiety, and pinpoint useful resources that could enhance their current life circumstances. This was significant as it demonstrated a process of problem-focused, coping that was used to cultivate the development of new skills and strategies that enhanced personal identity development. Reconstruction of veteran identity has been described (Jones, 2013; Olsen, Badger, \& McCuddy, 2014; Rumann \& Hamrick, 2010) in student veteran studies which commonly recommend social support, peer mentoring, and social networking as strategies to facilitate a new identity. Veterans in this study appeared to struggle more with the change in social identity than personal identity development.

Veterans in this study appeared to enhance life flow by overcoming a particular internalized or externalized task-oriented struggle that was encountered, which suggested the development of resilience or bouncing back from challenging life circumstances. This finding supports another student veteran study as an opportunity emerged to promote resilience-based supported education (Eakman et al., 2016) and interventions for veterans in transition. Resilience-based training programs for student veteran skill development have been described (Smith-Osborne, 2012b) and may be used to enhance life flow. Military training paradigms for resilience may also be adapted to support 
student veteran resilience skill development (Cornum, Matthews, \& Seligman, 2011). In sum, these findings suggest contextualizing student veteran transitions with a culturally relevant lens. A culturally-relevant lens supports veterans' challenges explained by perceived cultural differences within a new social environment. Although, the Kawa model has been previously used in case-studies that examined individualized narratives, this study demonstrated an ability of the Kawa to contextualize individual and collective actions, interactions, and processes of veteran transitions within a constructivist grounded theoretical study.

\section{Limitations}

While this constructivist grounded theory study demonstrated an emergent theoretical conceptualization of veterans' experiences co-constructed with veteran participation, some limitations need to be discussed. First, veteran experiences with transitioning likely vary at institutions in different regions with differing levels of resources, programs, and support devoted to veterans. To account for this limitation, we theoretically sampled veterans across two universities with variation in academic levels and enrollment in distance and in-classroom programs. Secondly, the majority of the student veterans interviewed within this study were comprised of a similar race. Only one participant self-identified as a minority. Differences in transition experiences may exist across veterans of differing racial and/or ethnic groups. Though we reached theoretical saturation with a sample of twelve veterans, the findings of this constructivist grounded theoretical study pertain primarily to the experiences of the sample interviewed. Further research is warranted to look at the differences in the transition experiences between gender and disability status. The incidence of Post 9/11 veterans with traumatic 
brain injury are relatively high compared to previous eras. The use of narrative methods to examine the life stories of student veterans in transition is warranted. Also, empirical research is needed to examine the relationship between social integration and social isolation in transitioning student veterans.

\section{Conclusion}

Student veterans transitioning to postsecondary education are a national concern for rehabilitation practitioners, educators, and researchers. Individuals assisting veterans must be responsive to the transition challenges experienced as the student veteran population increases to the projected enrollment of 5 million veterans by 2020 (American Council on Education, 2014). Veteran transition challenges are specific to the cultural context in which they are situated. Understanding veterans' experiences in military culture will assist in facilitate client-centered care by explicating the meaning shared within military service and between military service members. Efforts aimed at understanding military culture informs veterans of their value to student and civilian culture which promotes well-being. This study provides insights into the cultural context in which student veteran transition and how rehabilitation practitioners and educators can address veterans' needs from a lens focused on military culture.

Copyright (C Brian Tuan Gregg 2016 


\section{Chapter Six: Synthesis of Dissertation Results}

The three studies performed in this dissertation have sequentially added to each other, with examination of three distinct aspects of student veteran transitions to postsecondary education. The first study explored student veteran coping with transitions, the second study explored the lived experiences and meaning of transitions for student veterans, and the final study explored the cultural environments student veterans experience in transitions. In totality, the three studies provided a rich descriptive understanding of student veteran transitions and culminated in an emergent theoretical conceptualization for student veteran life flow in transition. Each study will now be briefly reviewed and its relationship to the emergent theoretical life flow model in study three. The chapter will conclude with a summarization of clinical implications and recommendations for practice and future research.

\section{Study One}

The first study surveyed student veterans' coping responses with transitioning to postsecondary education and aimed to determine the frequency of coping skill types reported. Thirteen student veterans with deployment experience participated in an anonymous online cross-sectional survey with items developed using Anderson, Goodman, and Schlossberg's (2012) Adult Transition Framework to explore their perceptions of their transition experience. Analysis of the frequency data indicated $38 \%$ reported difficulty managing finances, $93 \%$ utilized military training to manage stressors, and only $38 \%$ sought environmental supports provided by the university.

The first study described intra-individual responses to transitioning from the military to postsecondary education. Veterans reported difficulty managing financial 
problems in the transition which highlighted a functional area rehabilitation practitioners could support veterans in transition. Developing skills that supported budgeting and financial planning emerged as pragmatic considerations for preparing veterans before separating from the military. The majority of veterans in this study reported using the skills and training learned in the military to cope with their transition. This finding suggested a strong influence on personal skill, knowledge and ability development that occurred with the veteran during their time spent in the military. The major finding identified the broad categories of support and strategies that veterans frequently reported however, the finding could not account for the specific types of support or strategies each veteran primarily used.

Study one indicated veterans use environmental supports and resources provided by the university sparingly. Due to the limited responses into the specific skills veterans used to enhance their transition, the lack of explanation why campus resources were under-utilized, and the low response rate for the pilot study, we elected to perform a qualitative phenomenological study for the second investigation. The findings from study one gave rehabilitation practitioners a description of the challenges veterans experience and general categorizations for types of coping responses veterans commonly reported using for coping with a transition to postsecondary education. Study one also, provided a direction for the second study as the researchers needed to inquire about the meaning of the transition from the perspective of veterans as well as what barriers and facilitators affect engagement in university resources. Research questions there were derived from Study One that guided Study Two were: What are the lived experiences of discharged combat veterans transitioning to the occupation of education? What reasons 
do combat veterans report for using their Post 9/11 GI Bill? Is social support used by combat veterans transitioning to education? If so, how? What are combat veterans' attitudes towards the military as they transition to education? What do combat veterans think about the relationships with their peers? What are combat veterans' attitudes when they transition to education?

\section{Study Two}

Study Two described the lived experiences of student veterans transitioning from active military service to postsecondary education. Initial and follow-up phenomenological interviews were performed with 13 student veterans who had transitioned to postsecondary education with a history of military deployment. An overall essential meaning of 'emerging in college culture' was developed from three themes. The three themes that emerged were (1) repurposing military experiences for life as a student veteran, (2) reconstructing civilian identity, and (3) navigating postsecondary context and interactions. All of the themes were supported by rich textural and structural descriptions of student veteran's experiences.

In study two, veterans described their transition to postsecondary education in terms of a transactive process of standing in two worlds (military and civilian) simultaneously which required adaptation of performance skills and client factors developed in the military and used the educational context. Veterans retained military principles such as accountability and discipline to guide habits and strategies that supported new routines for academic success. Veterans also suggested aspects of their social identities were reconstructed in the postsecondary education context and deficits with socially interacting and relating to student peers influenced their social identities. 
Challenges experienced by student veterans in study two suggested problems consistent with perceived cultural differences that affected social interactions and participation with civilians and students.

Study two gave a deeper description of the phenomena of transition experienced by student veterans and provided insights into what the transition meant from their lived human experience. The challenges with social interactions suggested why student veterans did not regularly use environmental supports and resources provided by the university as veterans did not relate to individuals in the postsecondary education environment. The study also suggested that individual actions required by veterans to cope with the stressors of transitioning from the military were important, but collective actions with others was equally important.

Study two provided a richer understanding for the meaning of student veteran transitions and suggested a need to shift inquiry from a psychosocial perspective to a sociocultural perspective. In studies one and two, we learned more about the internalized and individualized actions of the client however, issues expressed by veterans consistently pointed towards perceived cultural differences experienced between the military, civilian, and student environment. Therefore, the research questions investigated in study three focused on examination of the veteran in context via a culturally-relevant approach. Individual and collective actions that were meaningful to veterans in the military were specifically explored via the Kawa model in order to theorize about student veteran transitions to postsecondary education. Research questions there were derived from Study Two that guided Study Three were: What is the meaning of the transition experience to postsecondary education for student veterans? 
How do student veterans describe their current physical and social environment? What life circumstances shape student veterans' transitions to postsecondary education? How do individual assets and liabilities influence student veterans' transitions to postsecondary education?

\section{Study Three}

In study three, student veteran transition experiences were examined with the Kawa (River) model (Iwama, 2006) as a novel approach to examining cultural environments veterans were contextually situated in. Twelve veterans were theoretically sampled and asked to develop diagrams of their transition experiences and complete a demographic questionnaire. After Kawa diagrams were developed, intensive interviews were performed to discuss the meaning ascribed to each river attribute depicted in veterans' diagrams. Intensive interviews were performed initially and as follow-up interviews. Interview data was analyzed using the constant comparative technique described by Charmaz (2006) and interviews ceased once theoretical saturation was attained.

The findings of study three explained veterans' transitions using the Kawa construct of life flow. The emergent theoretical conceptualization of student veterans life flow transition consisted to two major theoretical concepts: 'seeking understanding' and 'gaining stability' to shape life flow in transition from military culture, to civilian and student culture. Veterans shared understanding for their life circumstances experienced in each cultural environment by socially interacting with others perceived to be reaching out and connecting with them. Veterans also performed autonomous or individualized actions of gaining stability by using their skills, pinpointing useful resources, and 
cultivating the growth and expression of personal skills. These two theoretical processes simultaneously, enhanced each veteran's transition to postsecondary education and promoted harmony within veteran life flow over time.

Study three informed by the results of the prior two studies, sought to provide a theoretical conceptualization that explicated the properties of student veterans balancing themselves (gaining stability) and sharing meaning with others (seeking understanding) in the various cultural contexts experienced in their transition. The importance of collective actions and their meanings shared in the military were explicated in the development of the emergent model for student veteran life flow in transition. Military culture provided structure and meaning through processes focused on mission accomplishment. As the veteran left the military, the focus of collective mission accomplishment shifted to individual actions experienced in self-focused cultural environments which affected veterans in ways they were not initially prepared to understand or interpret. Negative life circumstances occurred in veterans' transitions but student veteran life flow improved by (1) individual actions of pinpointing useful resources and using/cultivating personal skills and (2) collective actions of others reaching out and connecting with veterans.

In studies one and two, the research questions primarily answered concerns addressed within the veteran via individual actions. Study three explicitly answered concerns of veterans experienced in context and provided an understanding in the differences between cultural environments via collective actions and processes. The findings from study three provided insights into the importance of military service to veterans and how it influences their worldview. The singular focus of military missions 
and the actions service members perform to accomplish missions were contrasted with civilian worker roles and academic pursuits performed by veterans. In this perspective, veterans carried a conflicting emic/etic perspective of being an insider with understanding of military experiences and seeing others as outsiders with little deference portrayed to those experiences. The Kawa model employed in this study was powerful in explaining veterans' perspectives in contrasting cultural environments. Using the emergent model derived from study three, student veteran transition experiences into postsecondary education can be explained with inclusion of their diverse human experiences. Veteran with invisible wounds of war (Tanielian \& Jaycox, 2008) experienced similar fluctuations in life flow during their transition as veterans who did not report any injuries sustained during active duty military service. This insight speaks to the importance of military culture in veterans' worldview of their transition. In many respects, Bridges (2004) assertion on transitions beginning from an ending narrowly applies to veterans in transition as veterans maintain the meaning of their military service indefinitely. What is clear by examining their transition to postsecondary education, is that they shape their life flow around others that they can continually share those military experiences with.

\section{Summary}

In this dissertation each study sequentially added to the next to construct a model that represents the influence of culture on student veterans' life flow when in transition from the military. The emergent model of student veteran life flow illuminates the cultural influence military experiences have on veterans in transition and the individual and collective actions taken to enhance life flow in the postsecondary education context. 
The student veteran life flow in transition model fosters an understanding of rehabilitation practitioners' role in supporting the transition process and promotes:

- Recognition of the influence of military culture on veteran life circumstances.

- Understanding the relationship between military culture and the choices veterans make to socially participate and utilize environmental supports.

- Practice as a rehabilitation practitioner affirming culturally-relevant and culturally-safe assessment.

- Inclusion of the diverse veteran military experiences in clinical practice to enhance rapport building and maximizing a therapeutic use of self for rehabilitation encounters.

- Reconciliation of purpose and meaning for veteran experiences by veteran advocates to navigate veterans toward environmental resources and activity engagement that support academic achievement.

\section{Implications for Practice}

Koenig et al. (2014) suggest practitioners incorporate the positive aspects of veterans' military and civilian social identities as an interactive social process to enhance veterans' cultural capacity for resilience when readjusting to the civilian world. In light of building veteran resilience to postsecondary education transitioning we suggest the following implications for culturally relevant practice:

- Iwama (2003) reminds us that in collectivistic social environments, "the self is oriented to adjusting and adapting to the external (social) environment, rather than controlling or altering the environs' to suit one's self' (p. 585). Veterans in this study expressed a view of self consistent with Iwama's thesis, therefore, 
rehabilitation practitioners and educators must first re-examine the theoretical lenses imposed on student veteran transitions to ensure their focus on well-being constitutes the collective beliefs veterans share. The emergent model constructed in this study considers the collective-orientated veteran in the postsecondary education social context and may be used in future studies for inquiry.

- Previous research suggests a need for transition coaching to holistically address transition needs of student veterans (DiRamio et al., 2008), therefore practitioners should consider quantitative as well as qualitative approaches to addressing perceived problem areas of student veterans. The Kawa model demonstrated power in explaining veteran problems areas that were further refined in our emergent model of student veteran life flow in transition. Modifications of this model or the Kawa may also explicate important factors assessed within veterans' narratives concerning various contexts in which practitioners engage veterans.

- Evaluation tools exist that practitioners can use to assess veteran transitions holistically, such as the Canadian Occupational Performance Measure [COPM] (Law et al., 2005). Goal setting may be initially addressed using the COPM as a holistic, client-centered assessment of veterans' occupational performance.

- Educators should liaison with local university counseling centers and veteran resource programs to assess the availability of veteran orientations, outreach programs, and/or remedial education courses. A definitive need exists to support resume building, job searching, academic counseling, and vocational assessment within the student veteran population. In particular, student veterans need assistance with identifying educational programs that correspond with their 
aptitude level in order to mitigate academic attrition. An assessment of veterans self-reported interest in specific vocations (Holland \& Messer, 2013) and current career thoughts (Sampson, Peterson, Lenz, Reardon, \& Saunders, 1996, 1998) would capture data on veterans' career interests used to guide academic program counseling.

- Student veteran support programs facilitate trust and connectedness across the campus community and promote an inclusive environment for veteran culture. An inclusive veteran transition program, may opt to incorporate the evaluation of life balance (Matuska, 2012) as it focuses on four domains (health, relationships, identity, and challenge) commonly identified as problem areas shared by veterans transitioning. The Life Balance Inventory (LBI) provides a reliable and valid outcome measurement tool for such an occupation-based program (Matuska, 2012).

- Supported education programs for veterans in higher education (Eakman et al., 2016) should foster resilience development consistent with current student veteran resilience programs available (Smith-Osborne, 2012b) or derivative of principles and concepts applied in military-based resilience programs (Cornum, Matthews, \& Seligman, 2011). These programs support the development of trait resilience that may be used to positively respond and adapt to negative life circumstances experienced in veteran transitions. University counseling support services may liaison with local campus veteran offices to offer resilience consultation, screening, and education in individual and group formats specific to veteran cohorts. 
- Veteran challenges with social interactions identify a performance skill practitioners can support for engagement in educational occupations (Simmons, Griswold, \& Berg, 2010; Søndergarrd \& Fisher, 2012). The Evaluation of Social Interaction (ESI) assessment tool (Fisher \& Griswold, 2010) provides a measurable behavioral outcome measurement for veteran communication skills in social interactions. The ESI is flexible for use within a natural context and may support veteran communication skill development following education of the lexical differences between military and student culture.

- Veterans in the final dissertation study consisted of participants that were completely transitioned from the military and no longer serving on Active Duty, National Guard, or Reserves. However, service members in the National Guard and Reserves need to be considered as their transition military experiences are likely different than their active duty counterparts. Post-9/11 era National Guardsmen and Reservists experience higher rates of mental health concerns following deployments which has been identified as a consequence of limited supports made available following deployment and demobilization periods (Hoge et al., 2006).

- The last recommendation concerns supporting veterans at the precipice of their transition. Veterans in this study experienced limited to no educational assistance, rather program officials from respective military Transition Assistance Programs (TAPs) recommended service members contact a VA education specialist once transitioned to a permanent civilian residence. This tactic reported was cursory at best and unhelpful for supporting service members in transition to 
school settings. We recommend a re-evaluation of TAPs services in the form of program evaluation and to consider inclusion of the following supports: (1) educational assistance from local university representatives, (2) VA education specialist consultation for Post 9/11 GI bill clarification, and (3) acculturation education and training regarding the differences in civilian and military culture. Emphasis in these supports should focus on clarifying the changes within these respective sociocultural environments and common challenges veterans must navigate to support life flow.

\section{Future Research}

The concept of this dissertation should be expanded for future research to evaluate transition challenges of active duty service members preparing to transition to civilian culture. In particular, research is needed to explore specific transitions of service members with health conditions and co-morbidities (e.g. poly-traumatic injuries, the poly-traumatic clinical triad, or traumatic brain injury). Case study research using the Kawa model is needed to describe life flow in active duty military service members preparing to transition from the military following combat deployment and/or injury.

Currently, there are no studies known to the author that use narrative methods such as the evaluation of life stories in relation to active duty service members with traumatic brain injuries. Other novel areas of military research includes military sexual trauma, intimate partner violence, and military family stress. Service members and dependent family members experiencing these significant problems rooted in military culture would likely provide expansive information into their experiences through the methods of qualitative research. Narrative based research would likely provide insights into the unique life 
circumstances of military service members and their family experiences following the host of interpersonal problems previously discussed and would also empower research participants to share those experiences.

The qualitative approaches described may be applied in the form of mixed method inquiry to assess quantitative outcomes as well. For instance, student veteran life flow may be researched in a cross-sectional study evaluating the relationship between social interaction skills and social isolation. Such a study would be powerful in describing aspects of student veteran transitions influencing life flow and to what extent social isolation is related social interaction. Life balance is another theoretical perspective that may be researched in active duty and veteran populations. However, the corresponding life balance inventory must first be validated in these populations in order to ensure an accurate and precise measurement of the four sub-scales anchored in perceived stress.

\section{Conclusion}

This dissertation began by exploring the attitudes and beliefs of veterans coping with transitions to postsecondary education and culminated by researching the cultural differences associated with veteran transition experiences. The student veteran life flow transition model symbolizes the powerful influence collectively-oriented military culture has on veteran transitions to postsecondary education. This emergent model serves rehabilitation practitioners in the process of navigating veterans to resources and strategies that improve their life circumstances for health, well-being, and academic

success. Practitioners engaging veterans should consider the aspects of military service as collective meaning inherent to veteran identity and well-being. In this respect, 
addressing veterans from a cultural lens drives positive growth in student veteran life flow in a dynamic process inclusive of purpose, meaning, and action.

Copyright $\odot$ Brian Tuan Gregg 2016 
APPENDIX A: Elsevier License to Reuse Kawa Content

\section{RightsLink}

Copyright

Clearance

Center

\section{Thank You For Your Order!}

Dear Brian Gregg,

Thank you for placing your order through Copyright Clearance Center's RightsLink service. Elsevier has partnered with RightsLink to license its content. This notice is a confirmation that your order was successful.

Your order details and publisher terms and conditions are available by clicking the link below:

http://s100.copyright.com/CustomerAdmin/PLF.jsp?ref=cc5f4bc1-0d274dba-a31e-94b8172b91c5

\section{Order Details}

Licensee: Brian Gregg

License Date: Dec 11, 2015

License Number: 3765940181262

Publication: Elsevier Books

Title: Rehabilitation

Type Of Use: reuse in a thesis/dissertation

Total: 0.00 USD

To access your account, please visit https://myaccount.copyright.com.

Please note: Online payments are charged immediately after order confirmation; invoices are issued daily and are payable immediately upon receipt.

To ensure that we are continuously improving our services, please take a moment to complete our customer satisfaction survey.

\section{B.1:v4.2}

+1-855-239-3415 / Tel: +1-978-646-2777 customercare@copyright.com http://www.copyright.com 


\section{APPENDIX A Continued}

$12 / 42015$

RightsLink Printable Licerse

\section{ELSEVIER ORDER DETAILS}

Dec 04, 2015

This is an Agreement between Brian Gregg ("You") and Elsevier ("Elsevier"). It consists of your order details, the terms and conditions provided by Elsevier ("Elsevier"), and the payment terms and conditions.

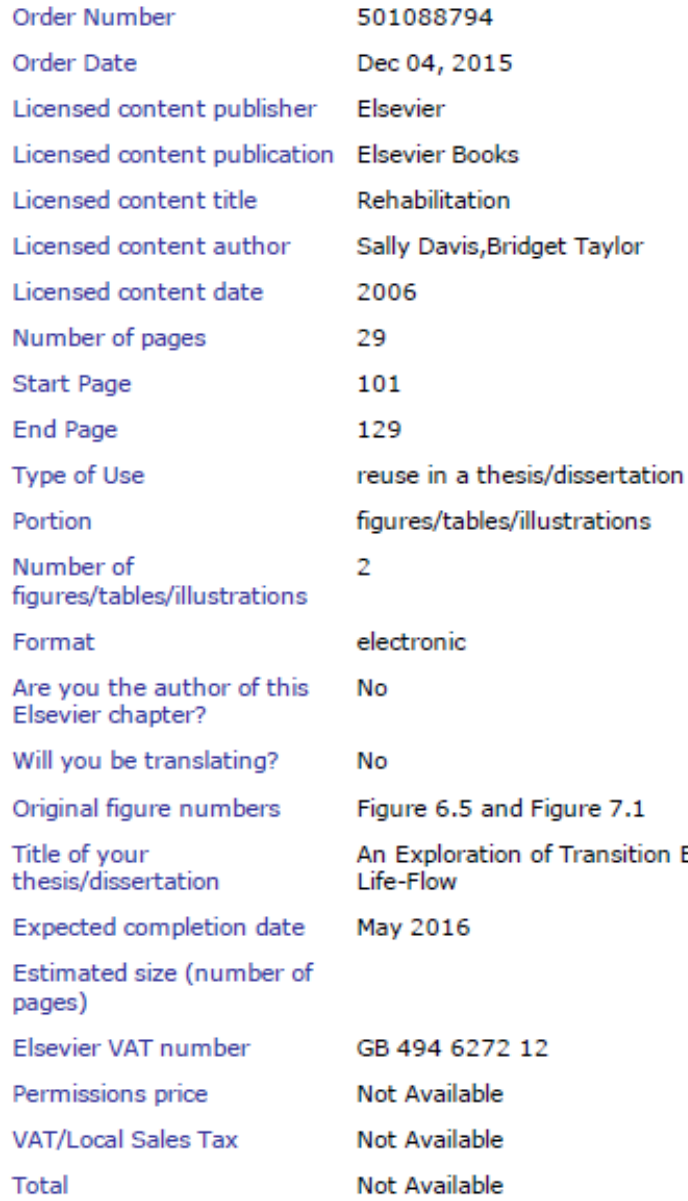


APPENDIX B: Demographic Questionnaire

\section{$\underline{\text { Student Veteran Demographic Form }}$}

\begin{tabular}{|c|lc|}
\hline 1 & Please indicate your gender? & Male or Female \\
\hline 2 & What is your age? \\
\hline 3 & Please identify your ethnicity? \\
\hline 4 & Please indicate your student status? Undergraduate, Graduate or Post Graduate \\
\hline 5 & What year did you enroll in the university? \\
\hline 6 & What armed service branch did you discharge from? \\
\hline 7 & $\begin{array}{l}\text { What was your COMPO military status? Active duty, Reserve, National } \\
\text { Guard }\end{array}$ \\
\hline 8 & How many times have you deployed? & Yes or No \\
\hline 9 & What was your length(s) of deployment? \\
\hline 10 & Are you affiliated with the Student Veterans Association? \\
\hline 11 & Do you utilize the Veteran Resource Center? \\
\hline 12 & Do you commute to school? If so, how far (miles)? \\
\hline 13 & Do you have a vocation and go to school? & \\
\hline 14 & What is your major? & Yes or No, what is your vocation? \\
\hline 15 & What is your cumulative GPA? & Yes or No \\
\hline 16 & How many universities have you attended? \\
\hline 17 & Do you have a service connected disability for VA services? \\
\hline 18 & Are you married? \\
\hline 19 & Do you have any children? \\
\hline
\end{tabular}




\section{APPENDIX C: Kawa Inquiry Protocol}

1) The researcher invites the participant to draw a picture or diagram of a river depicting their current state of being in the transition to postsecondary education. Prompts to the participant would include:

a) "Would you mind drawing a picture of a river as a metaphor for your life at the present time?" or

b) "Suppose your river is your life at this point in time, can you continue your drawing to make it represent your present situation?"

2) The researcher discusses the relevance of features with the participant then introduces components of the formal model such as rocks, river side walls, drift-wood, and water courses.

a) Example, "if rocks of various sizes stand for problems or challenges that sit in your river and block or slow down the water flowing, what would they be.....can you draw them into your river?"

b) The participant may elect to draw/insert objects that are not part of the standard Kawa Model protocol into their river drawing. The researcher should permit this to happen, planning then to ask the participant to explain those objects and their relevance to the overall river/life flow narrative.

3) The researcher affirms the meaning of the magnitudes, relative locations, and relationships that make up the client's river.

a) Example: "what is this rock here and why is this one so big? This rock is wedged between these other two large rocks and all three are resting against this part of the river wall.....why?"

4) The researcher collaborates with participants to prioritize problems and challenges, personal assets and liabilities, transient and environmental factors to discuss their meanings.

5) The researcher concludes the activity and clarifies any questions the participant may have.

6) The researcher provides demographic questionnaire for participant to complete.

\section{Additional Probing Questions for Student Veteran Transition Experiences}

- How would you describe the current flow of your river?

- Tell me about your river side walls and bottom. 


\section{APPENDIX C: Continued}

- How would you characterize the support provided in your transition? Tell me more.

- When, if at all, did you first experience any support from your university?

- If so, what was it like? What did you think then? How did you happen to engage in the university support provided?

- Who, if any, influenced your actions? Tell me about how they influenced you?

- Could you describe any events represented in your rocks that have affected your transition?

- Who, if anyone, was involved? When was that? How were they involved?

- What positive changes have occurred in your life flow? How, if at all, has the university been involved?

- What negative changes, if any, have occurred in your life flow? How, if at all, has the university been involved?

- How would you characterize your personal strengths flowing in your river?

- How would you characterize any personal liabilities flowing in your river?

- What if anything, could the university do to support any liabilities identified in your river?

- Could you describe the most important lessons learned when transitioning to your university?

- What do you think are the most important ways universities can support student veterans in transition?

*Note: since this will be an intensive interview, there may be additional questions that arise resulting from responses given by participants. However, the above questions will be asked of all participants. 
APPENDIX D: Kawa Diagram Zane

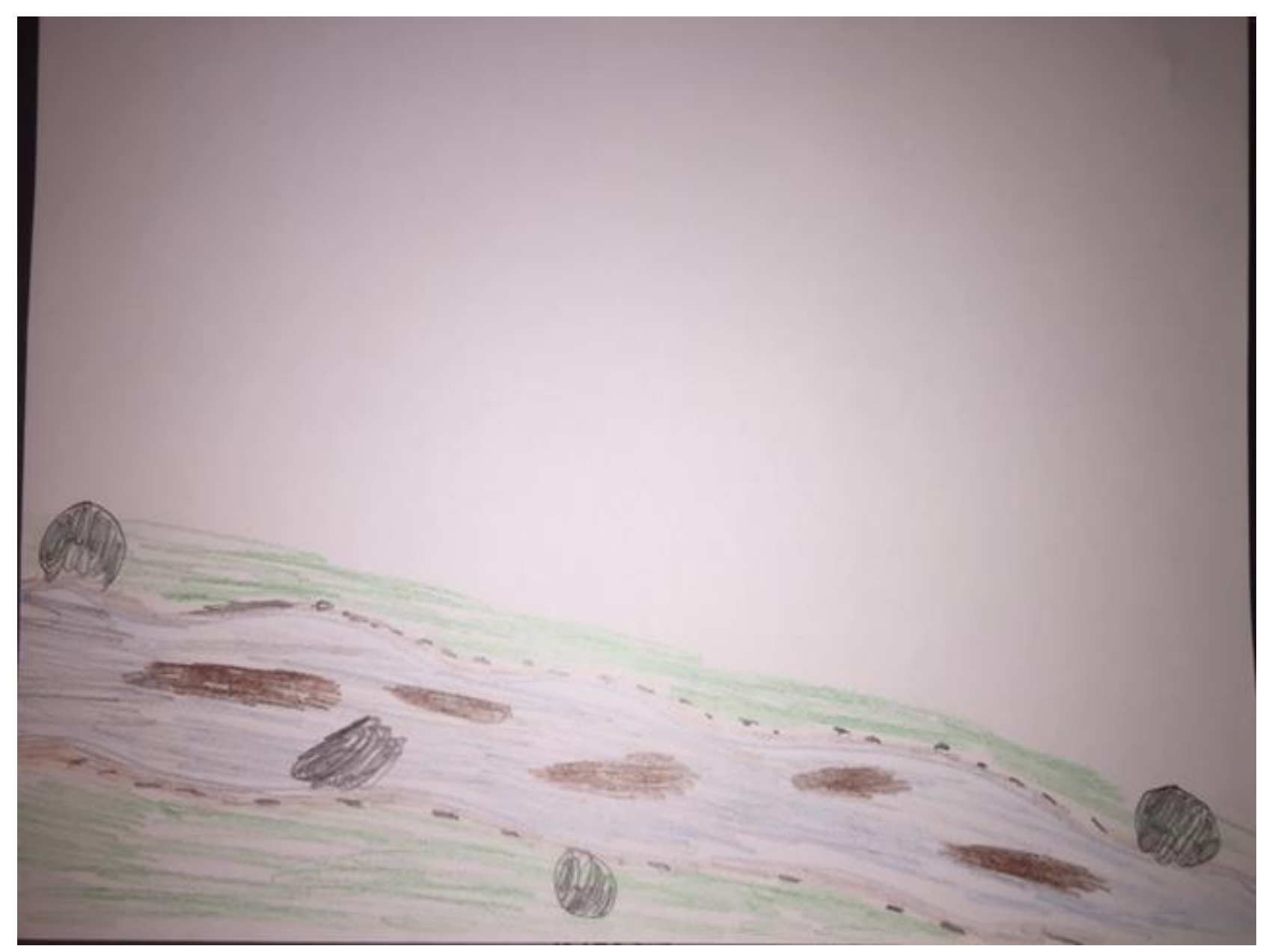



APPENDIX F: Kawa Diagram James

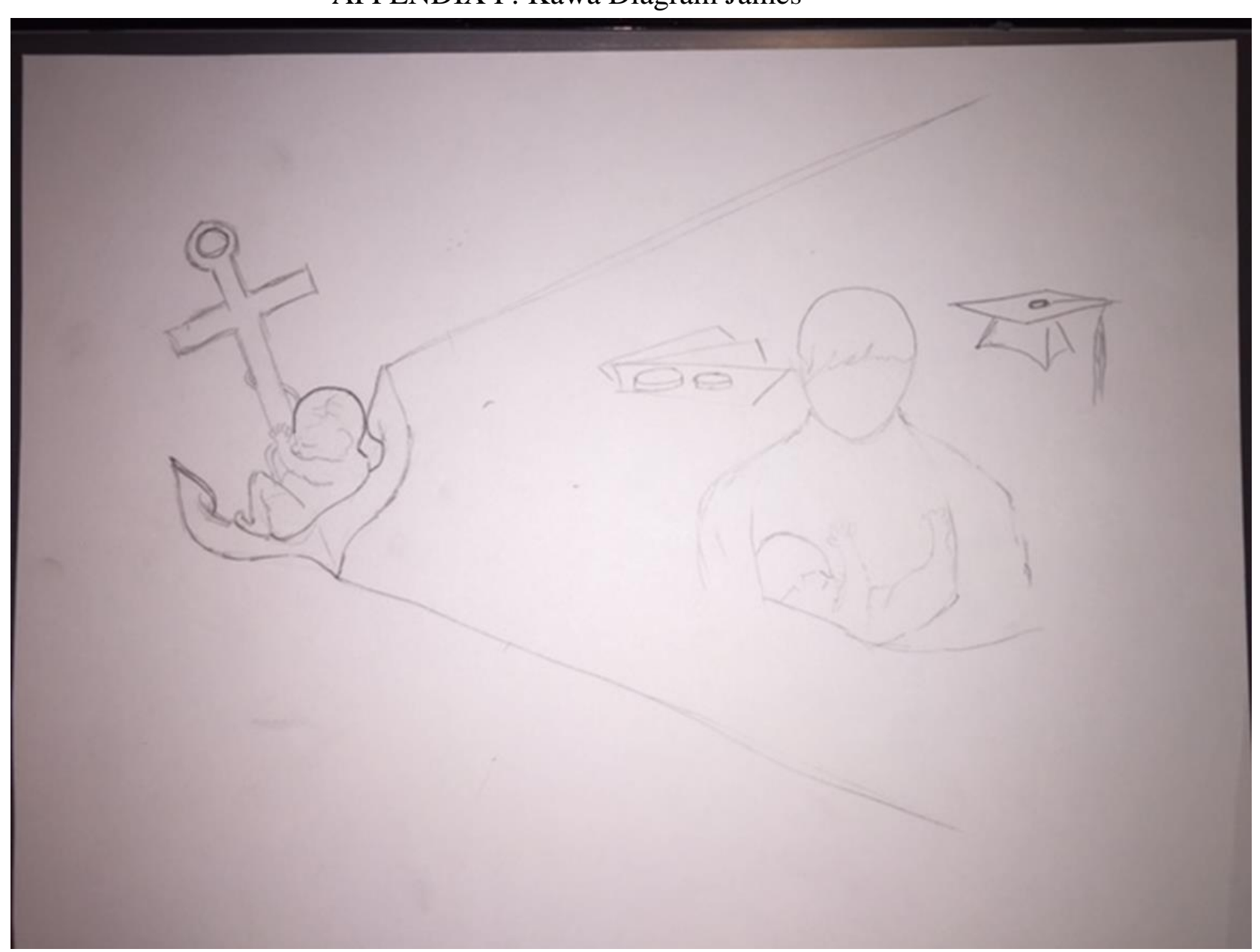


APPENDIX G: Kawa Diagram Jim

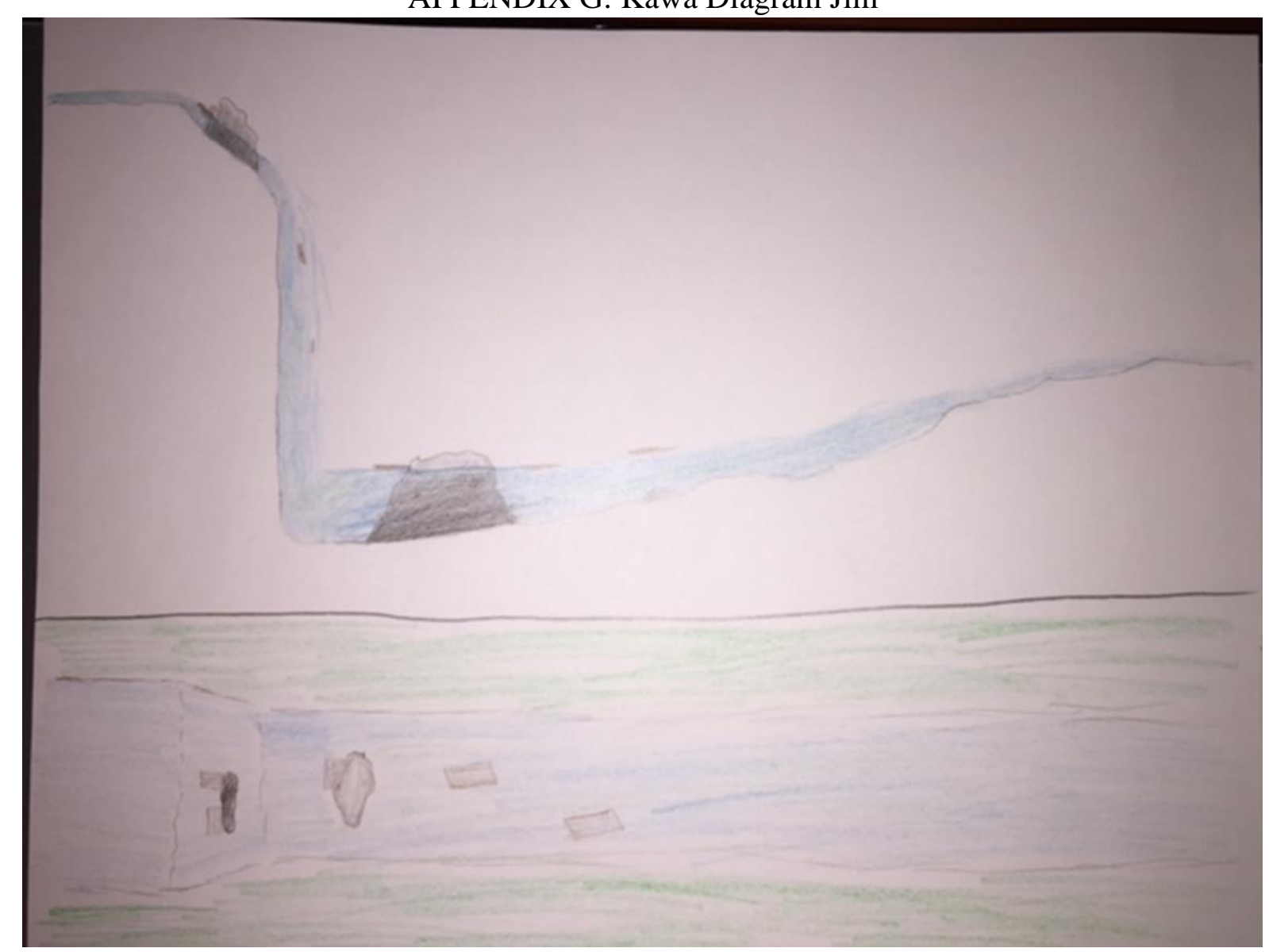


APPENDIX H: Kawa Diagram Tim

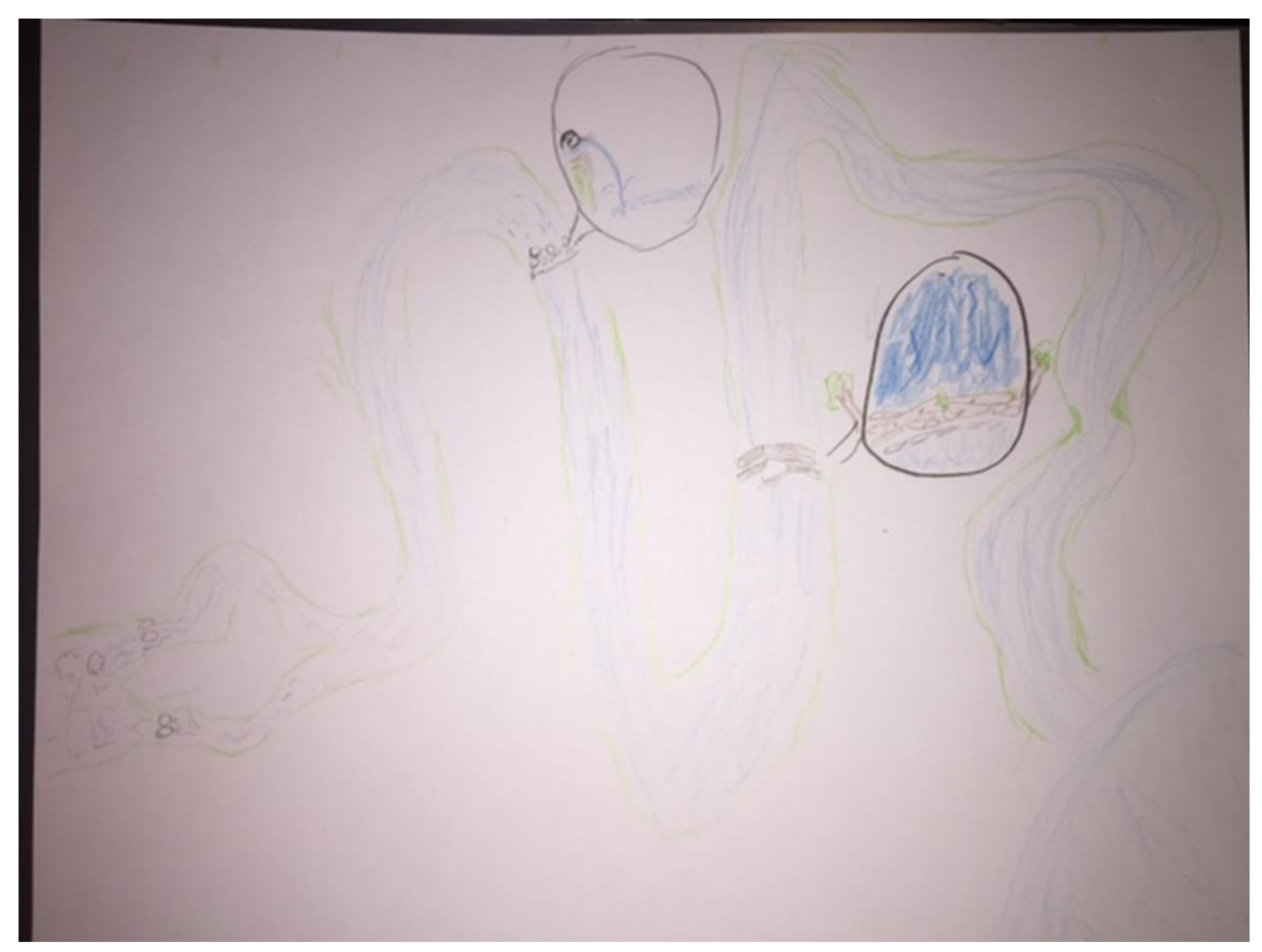


APPENDIX I: Kawa Diagram Brad

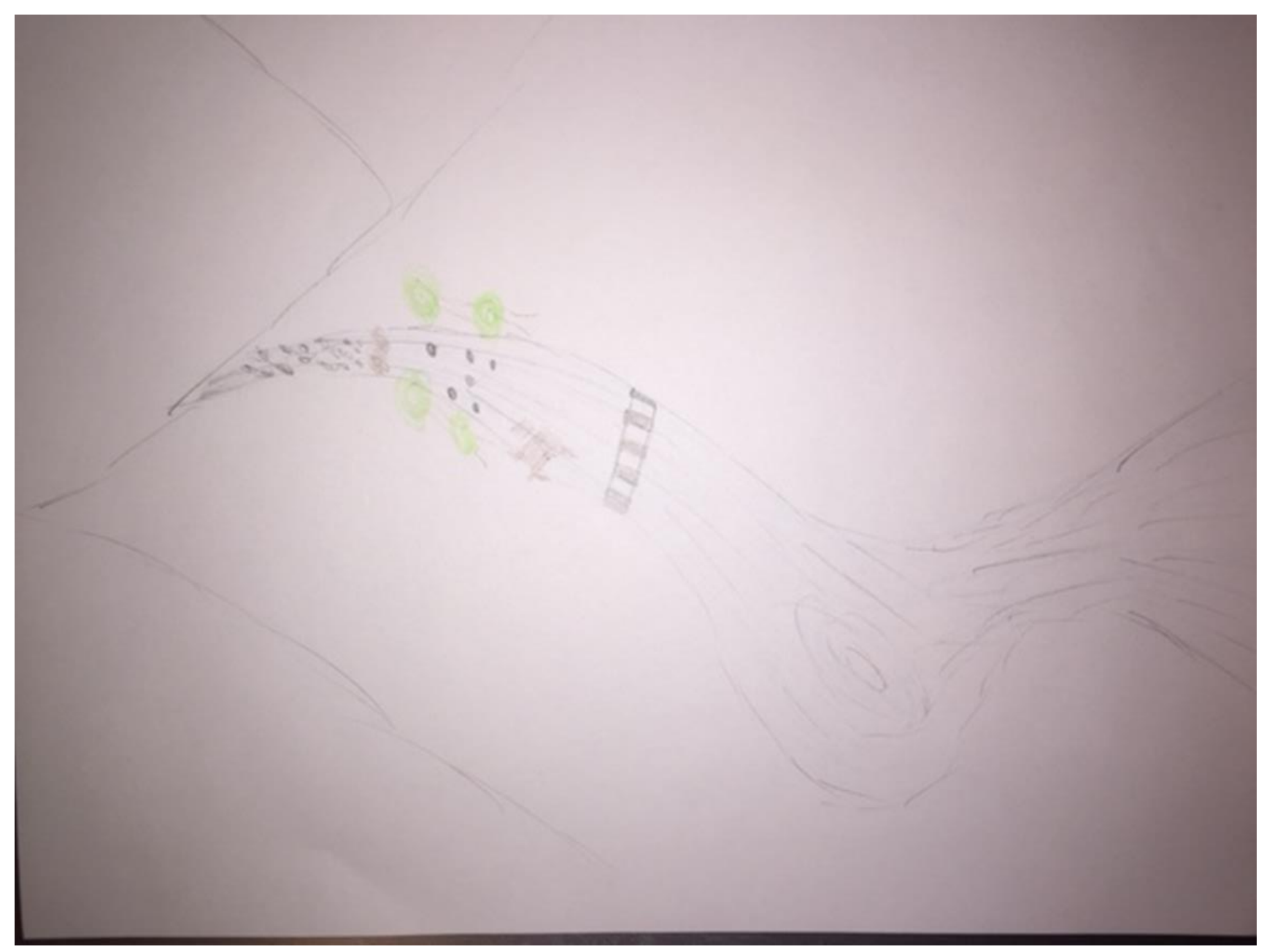


APPENDIX J: Kawa Diagram Andy

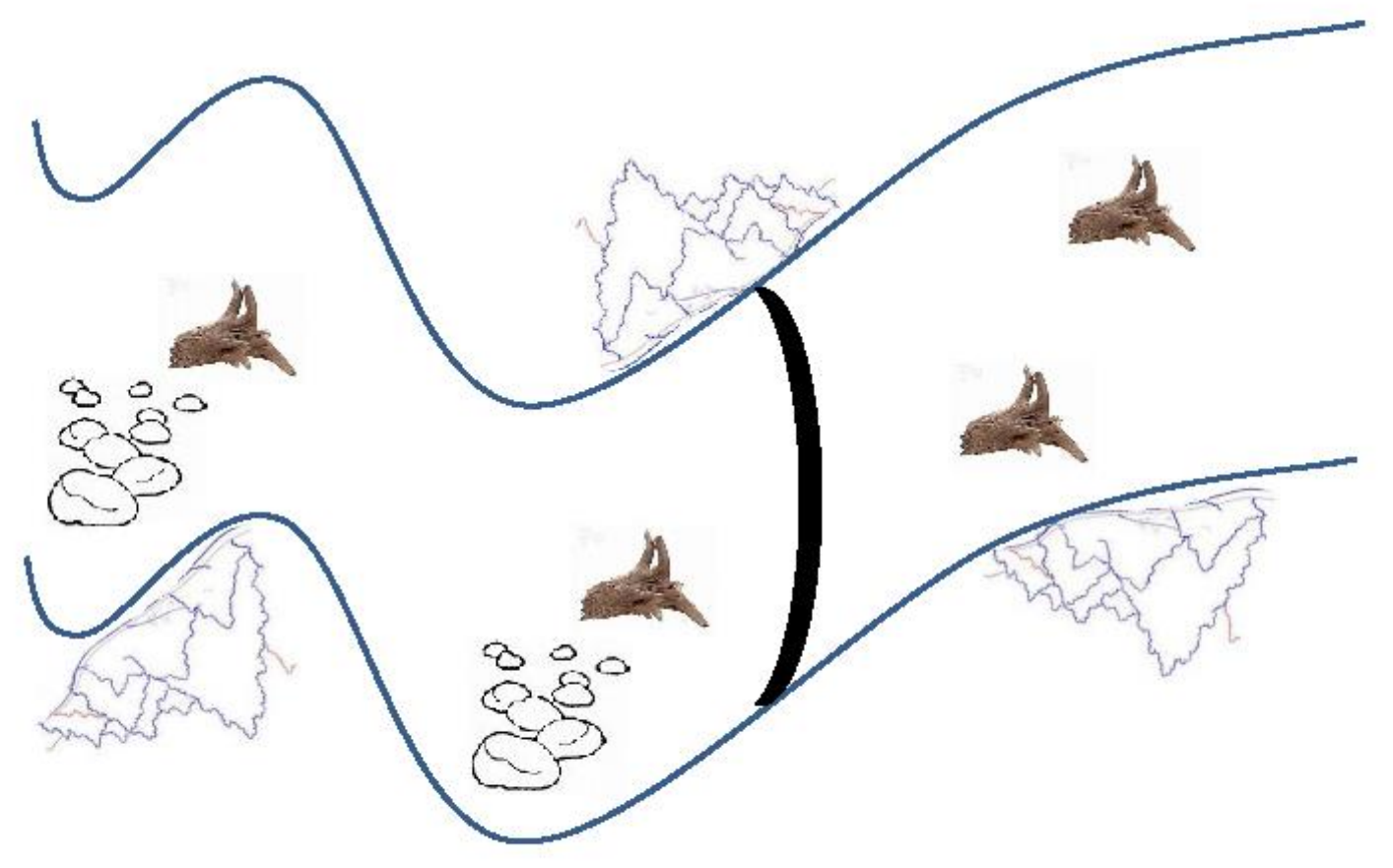


APPENDIX K: Kawa Diagram Kelly

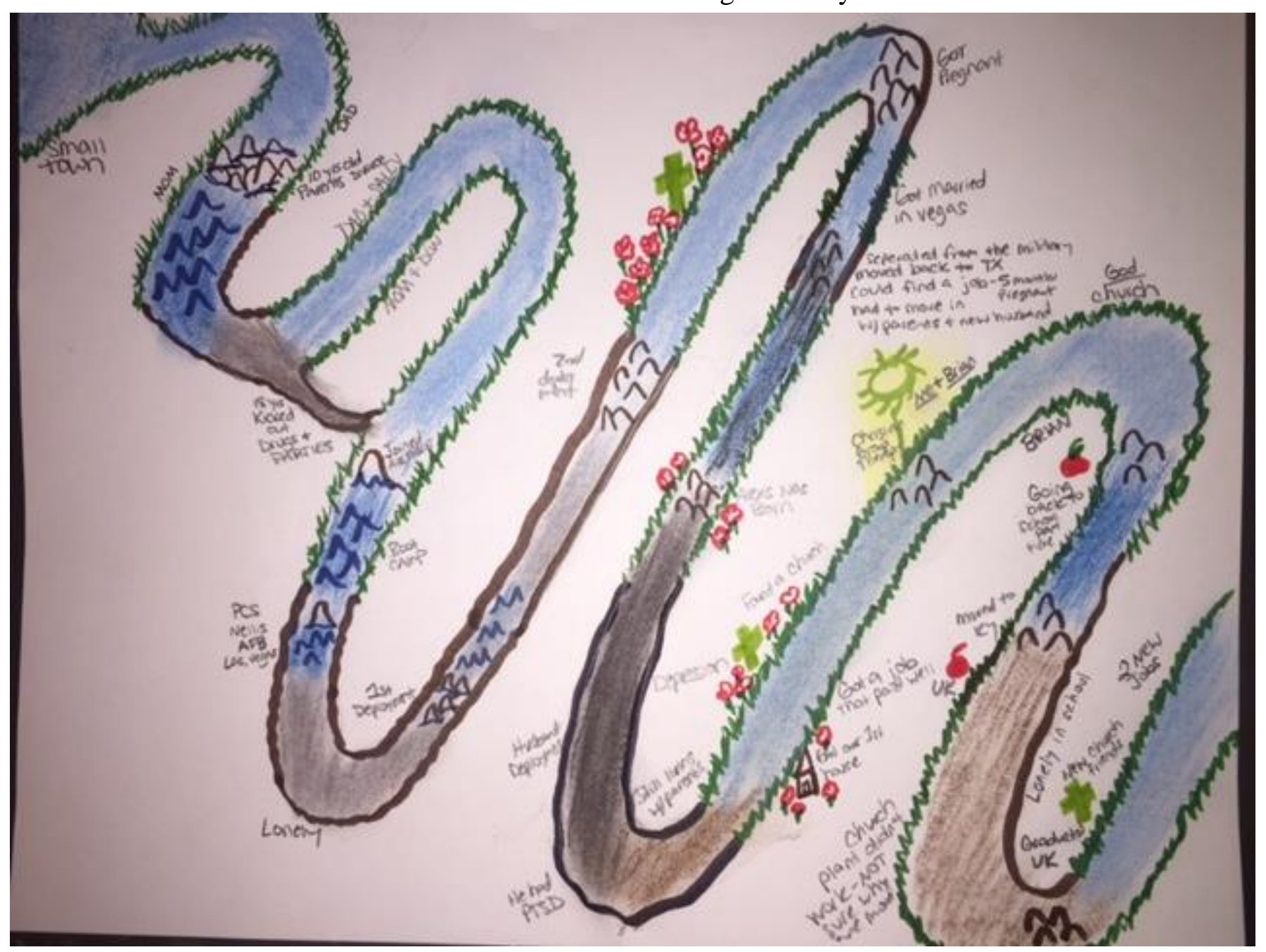


APPENDIX L: Kawa Diagram Shelly

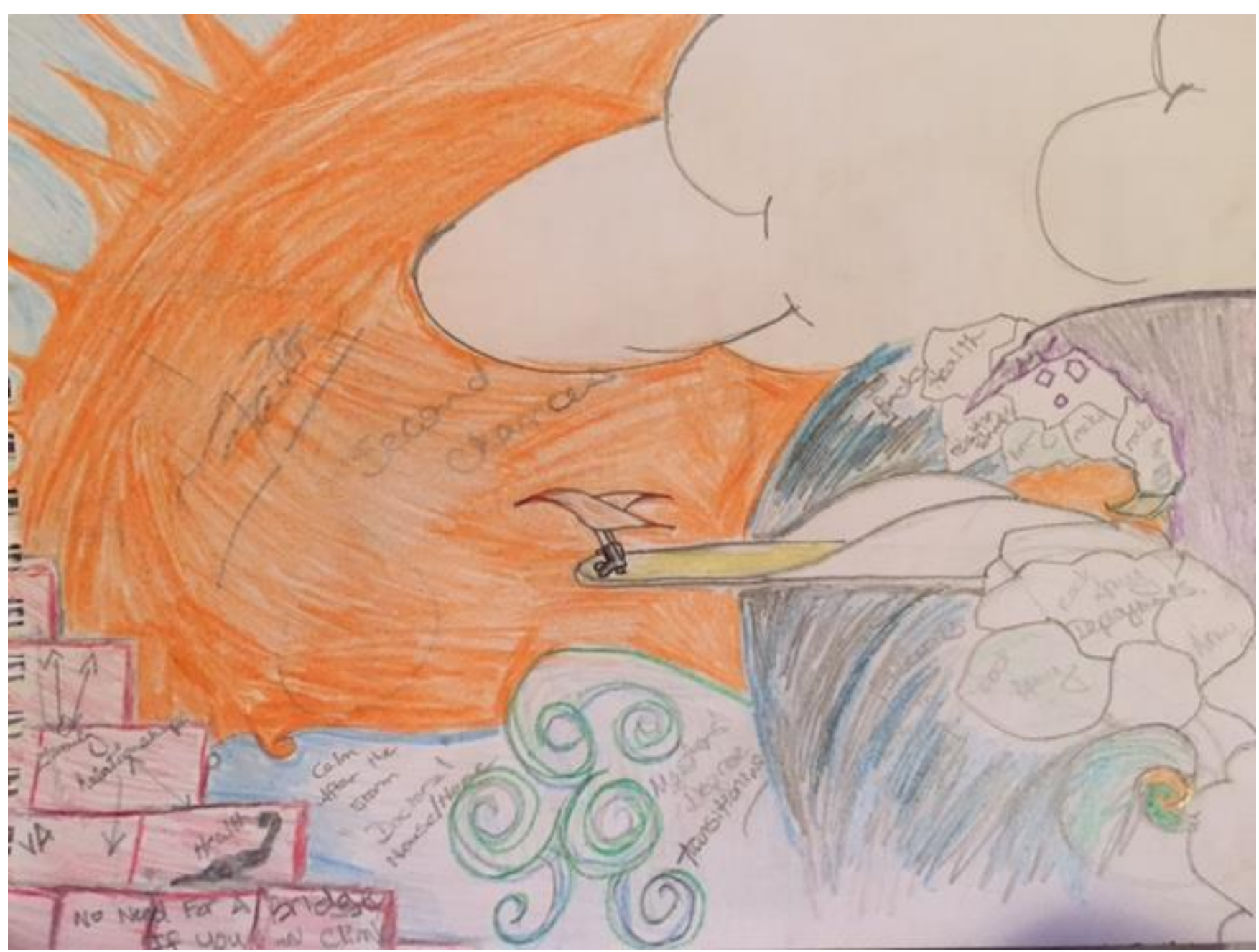




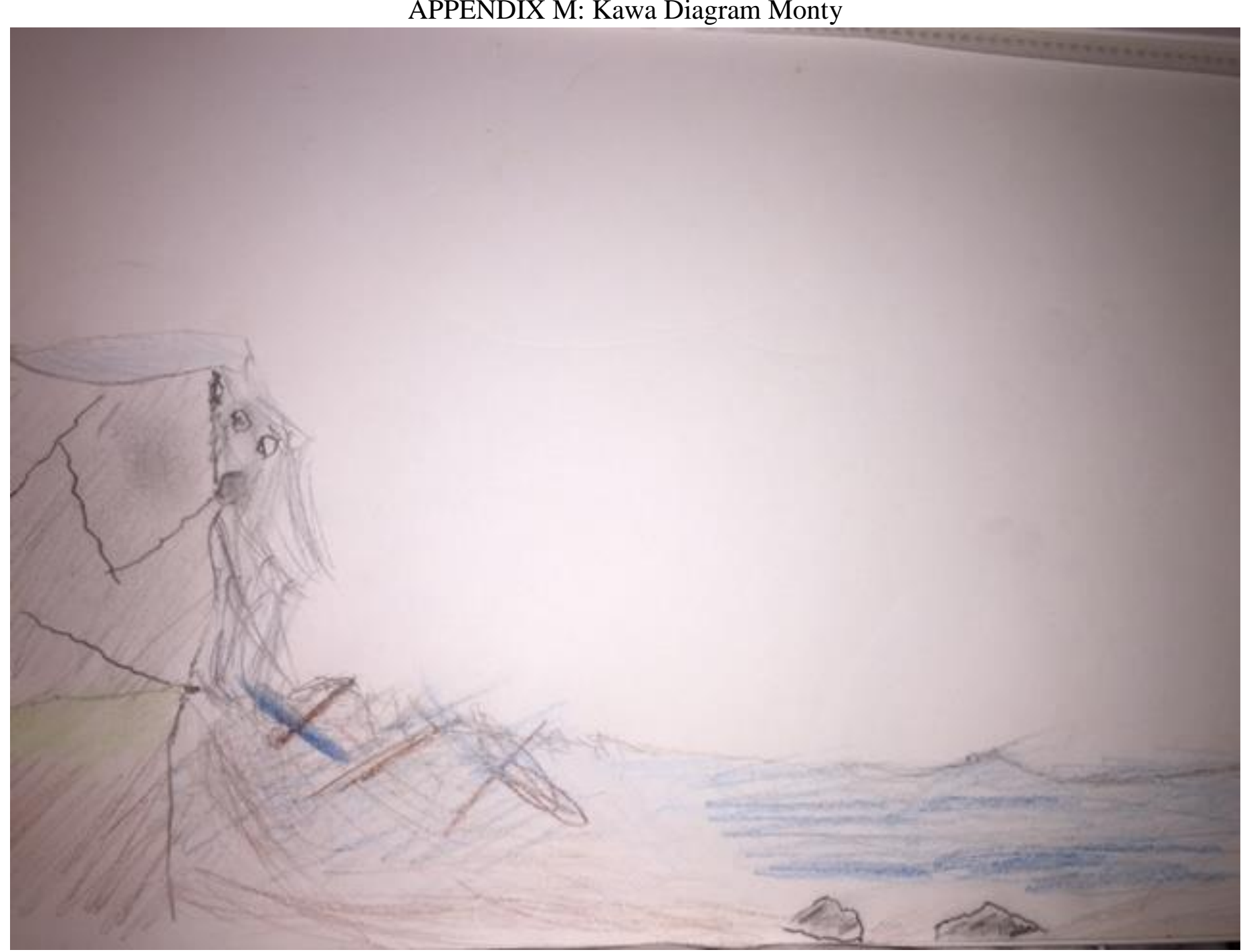


APPENDIX N: Kawa Diagram Rachel

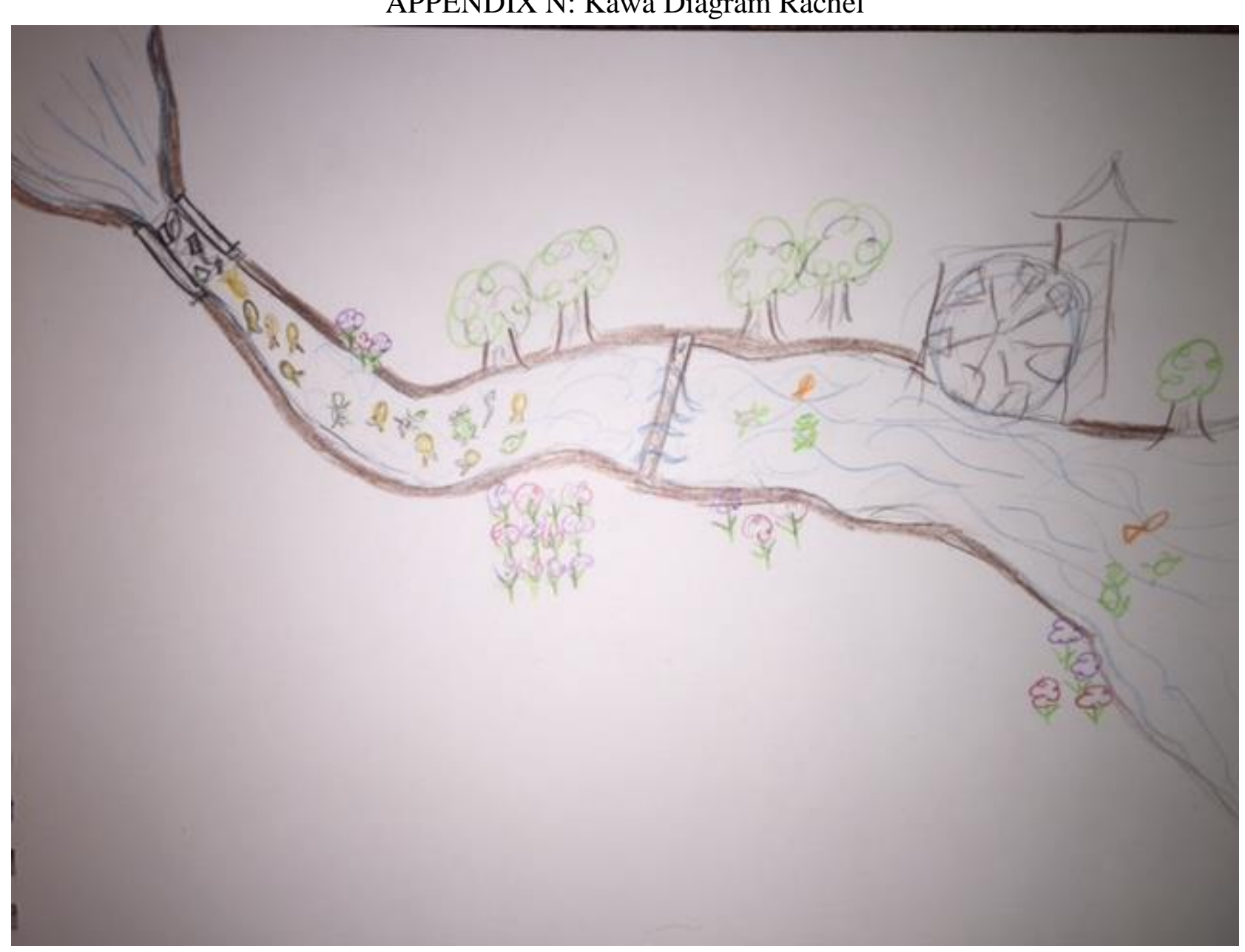


APPENDIX O: Kawa Diagram Ron

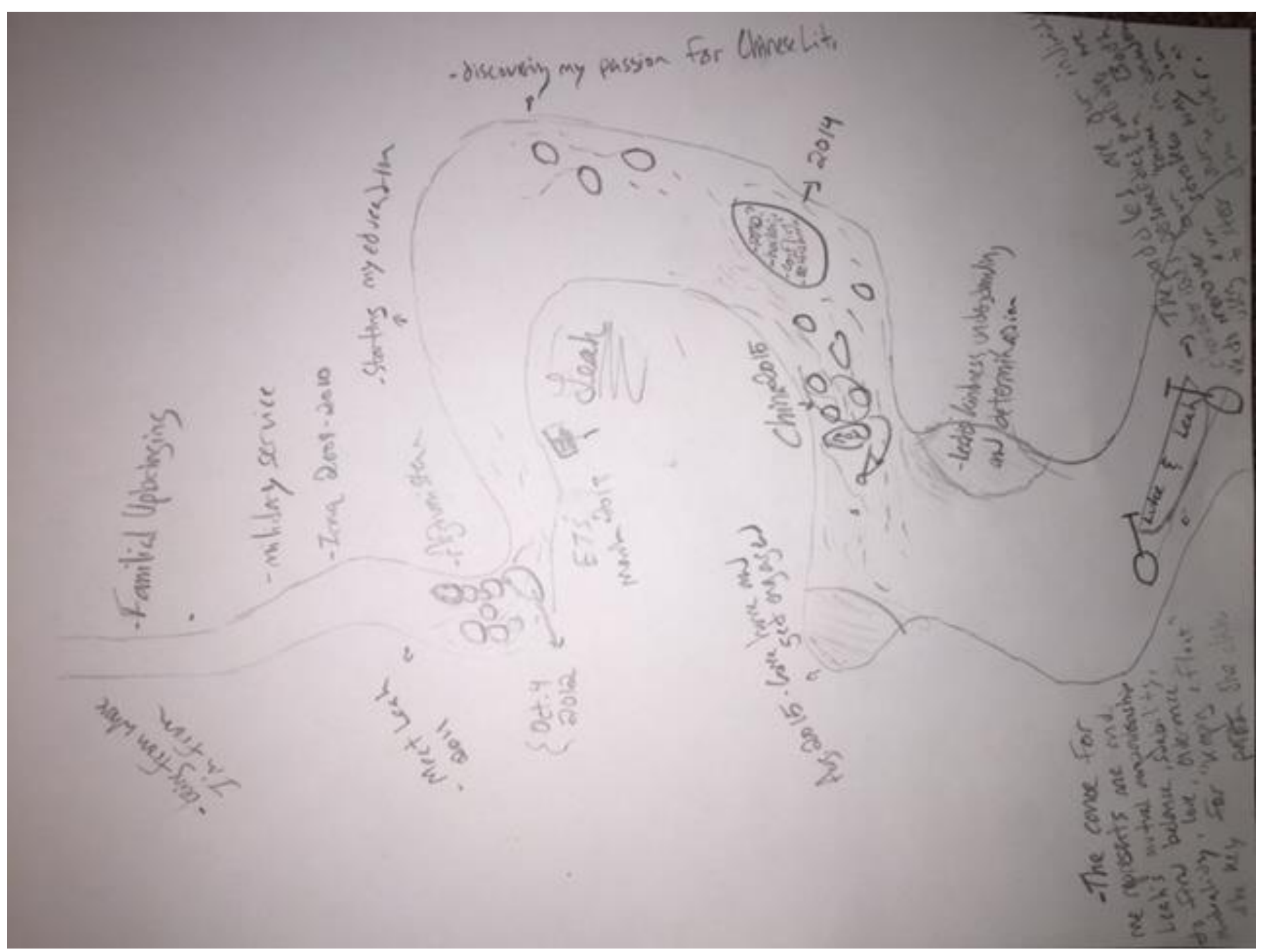




\section{APPENDIX P: Constructivist Coding Process}

\begin{tabular}{|c|c|c|c|}
\hline $\begin{array}{l}\text { Constructivist Coding Process } \\
\text { Raw Data }\end{array}$ & Initial Coding & Focused Coding & Theoretical Coding \\
\hline [Evan] One of my first classes here, I was in & Accommodating veteran & Needing understanding & The process of seeking \\
\hline this massive auditorium with over five-hundred & Being sensitive to outsiders & & life flow \\
\hline seats, and there was only maybe a hundred & Explaining veteran problems & & \\
\hline people in the class. But the professor wanted & Feeling supported by peers & & \\
\hline everyone to sit in the front six rows and fill up & Finding an empathetic counselor & & \\
\hline every seat because he did not want to yell at & Finding those who know combat & & \\
\hline everyone in the class. That's understandable, & Having a hard time with family & & \\
\hline but I can't do that. So, I had to sit at the eighth & Informing others & & \\
\hline or ninth row because I have to be able to watch & Lacking empathy from peers & & \\
\hline everybody. Cause if somebody goes to do & Lacking trust for the VA & & \\
\hline something they shouldn't I want to be prepared & Lacking veteran understanding & & \\
\hline for it and know how to react. So, getting & Periodic occurance & & \\
\hline professors to understand them and to not force & Receiving specialized training & & \\
\hline $\begin{array}{l}\text { them to do something that they would not be } \\
\text { okay with. }\end{array}$ & $\begin{array}{l}\text { Recognizing schools who } \\
\text { understand veterans }\end{array}$ & & \\
\hline & Recommending understanding & & \\
\hline [Kelly] I don't think any of them understood & Understanding not reciprocated & & \\
\hline me. I don't think any of them understood my & Being confused & Identifying vulnerabilities as a & \\
\hline story I have had so much stuff going on in my & Being less judgmental & student & \\
\hline & Dealing with life after graduation & (28 Codes) & \\
\hline [James] The main thing is juggling family life & Dealing with novel responsibilitie & & \\
\hline and all of that in a short amount of time... I & Difficulty staying awake & & \\
\hline mean, I have never rented an apartment. I & Establishing spending practices & & \\
\hline have always been on a ship. So, all of my stuff & Experiencing financial troubles & & \\
\hline was in this coffin locker. So, that is my river, it & Expressing frustrations & & \\
\hline is a transition from a mentality wise, from an & Feeling differences within & & \\
\hline 18 year old just getting out of the nest to a 30 & veteran groups & & \\
\hline & Feeling unconnected to campus & & \\
\hline & Feeling unnerved & & \\
\hline & Having standards met & & \\
\hline [Tim] I didn't have the resilience necessary & Lacking resilience & & \\
\hline that I needed to bounce back from starting the & Needing resiliency & & \\
\hline semester late. & Overwhelmed by cultural change & & \\
\hline
\end{tabular}




\section{APPENDIX P (continued)}

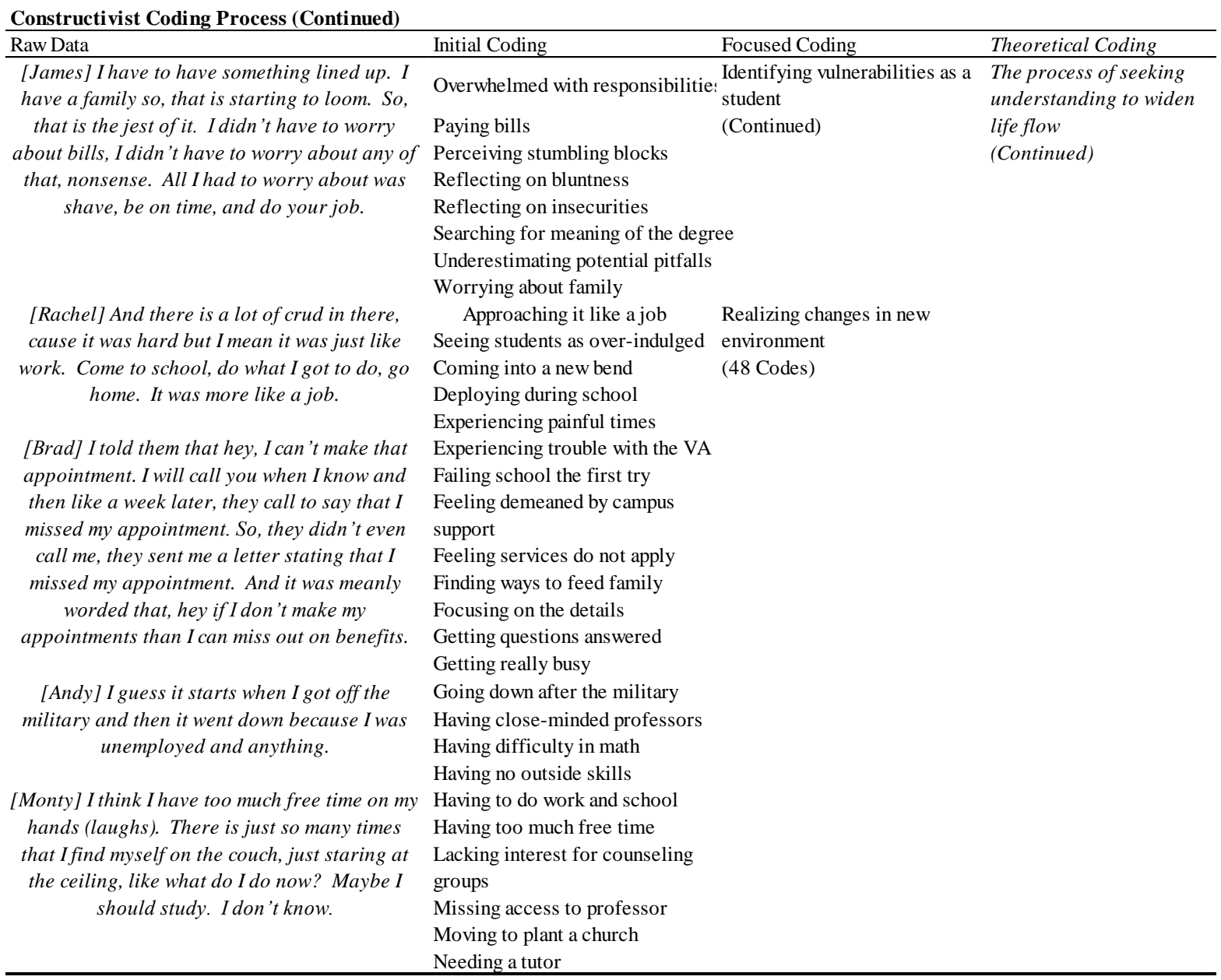




\section{APPENDIX P (continued)}

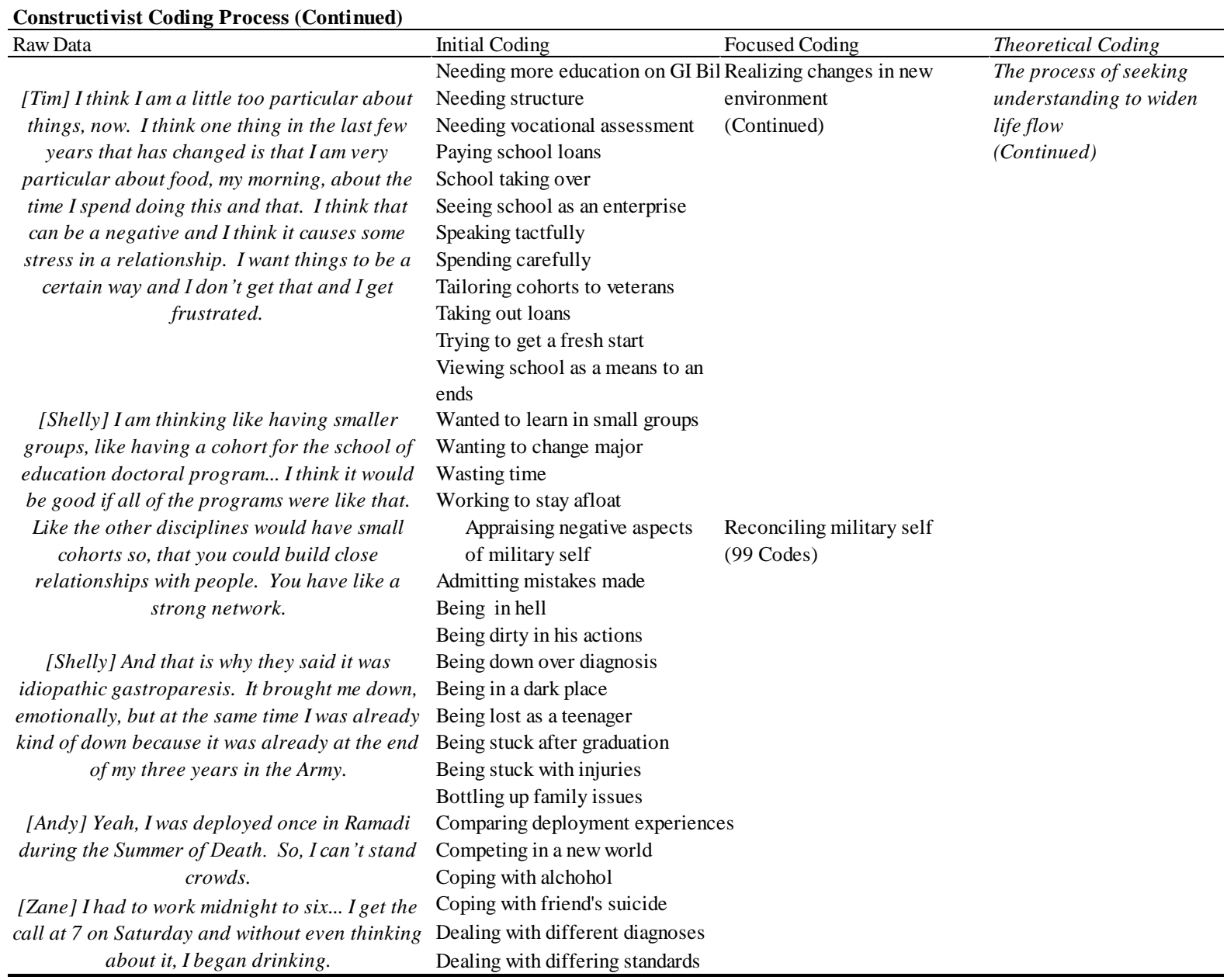




\section{APPENDIX P (continued)}

\begin{tabular}{|c|c|c|c|}
\hline Raw Data & Initial Coding & Focused Coding & Theoretical Coding \\
\hline $\begin{array}{l}\text { [Monty] I've had a lot of anxiety. Like a lot of } \\
\text { anxiety. Like around people and crowds. New } \\
\text { places. Certain times in the night. I actually } \\
\text { went and saw some help for it and they tried to } \\
\text { tell me that I had PTSD. I tried to punch him in } \\
\text { the face. } \\
\text { [Brad] Kind of delayed or I don't know what it } \\
\text { was, but about six months after I got back for } \\
\text { about two years it was bad. I went for about } \\
\text { six months-worth of counseling. And then I did } \\
\text { a few months of group therapy after that. } \\
\text { [Monty] So, everything gets kind of muddy } \\
\text { because Ijust... there were times that I enjoyed } \\
\text { what I was doing and sometimes I just look } \\
\text { back at it and I am... [Hand gestures toward } \\
\text { himself] you are a sick man Monty, you are a } \\
\text { sick man Monty... So, it's not like I look back on } \\
\text { it with regret or anything, you know. It's just } \\
\text { that things got a little dirty. }\end{array}$ & $\begin{array}{l}\text { Dealing with PTSD } \\
\text { Developing a lifestyle of hatred } \\
\text { Doing normal activities } \\
\text { Examining anger issue } \\
\text { Experiencing a flashback } \\
\text { Experiencing a friend's suicide } \\
\text { Experiencing delayed symptoms } \\
\text { Experiencing health complications } \\
\text { Expressing frustration with the VA } \\
\text { Expressing outlets for anger } \\
\text { Finding self in turbulent times } \\
\text { Gaining weight from meds } \\
\text { Getting dirty } \\
\text { Having medicinal complications } \\
\text { Having marital discord } \\
\text { Having service-connected } \\
\text { disabilities } \\
\text { Internalizing a freind's suicide } \\
\text { Internalizing grief } \\
\text { Isolating self } \\
\text { Living a facade } \\
\text { Living with PTSD } \\
\text { Losing his humanistic side } \\
\text { Needing something for self } \\
\text { Organizing worries } \\
\text { Percieved brokeness } \\
\text { Picking up the pieces } \\
\text { Prioritizing health first } \\
\text { Questioning alternative support } \\
\text { Rattling nerves } \\
\text { Reaching his breaking point } \\
\text { Thinking about long term disability } \\
\text { Turning to alchohol }\end{array}$ & $\begin{array}{l}\text { Reconciling military self } \\
\text { (Continued) }\end{array}$ & $\begin{array}{l}\text { The process of seeking } \\
\text { understanding to widen } \\
\text { life flow } \\
\text { (Continued) }\end{array}$ \\
\hline
\end{tabular}




\section{APPENDIX P (continued)}

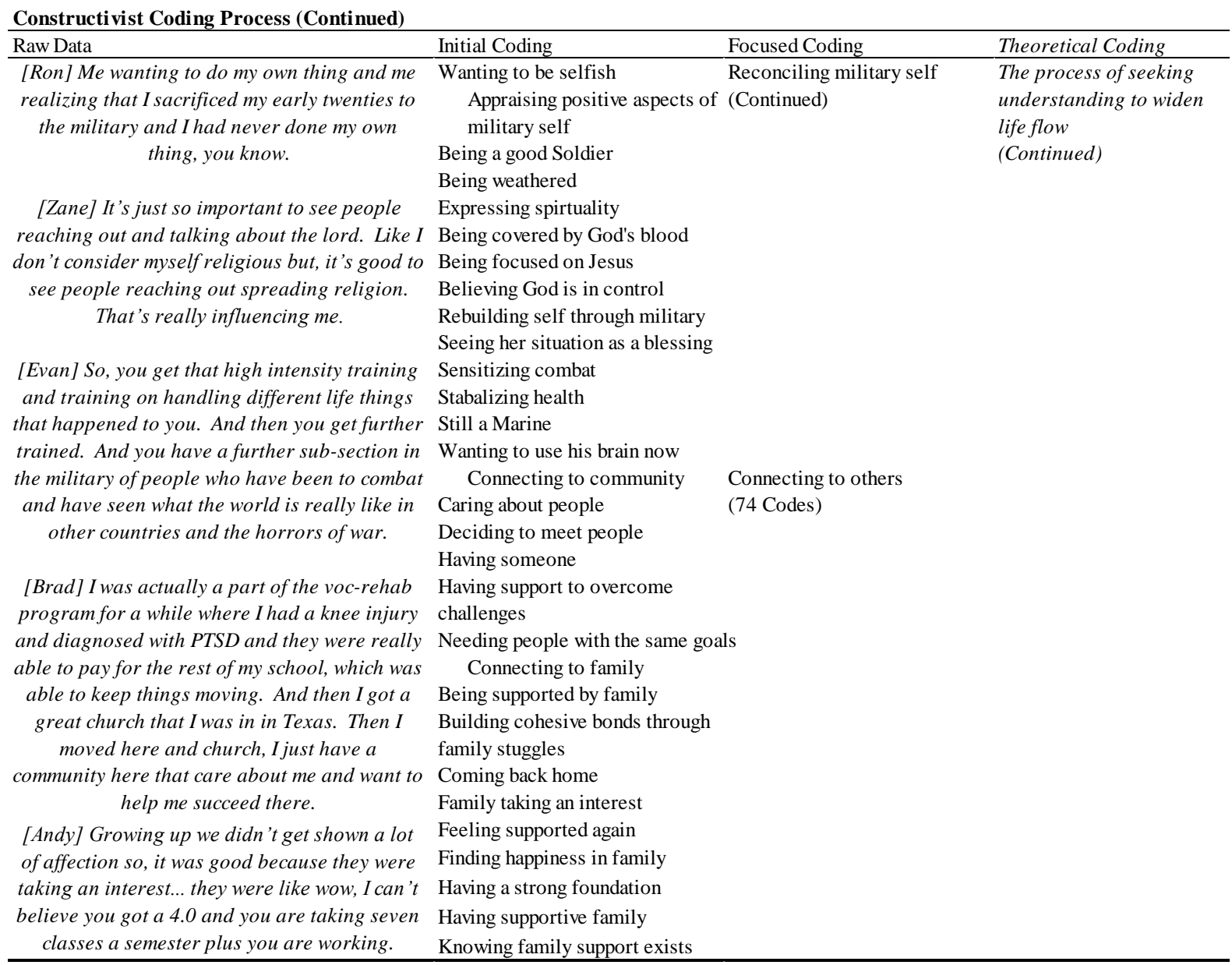




\section{APPENDIX P (continued)}

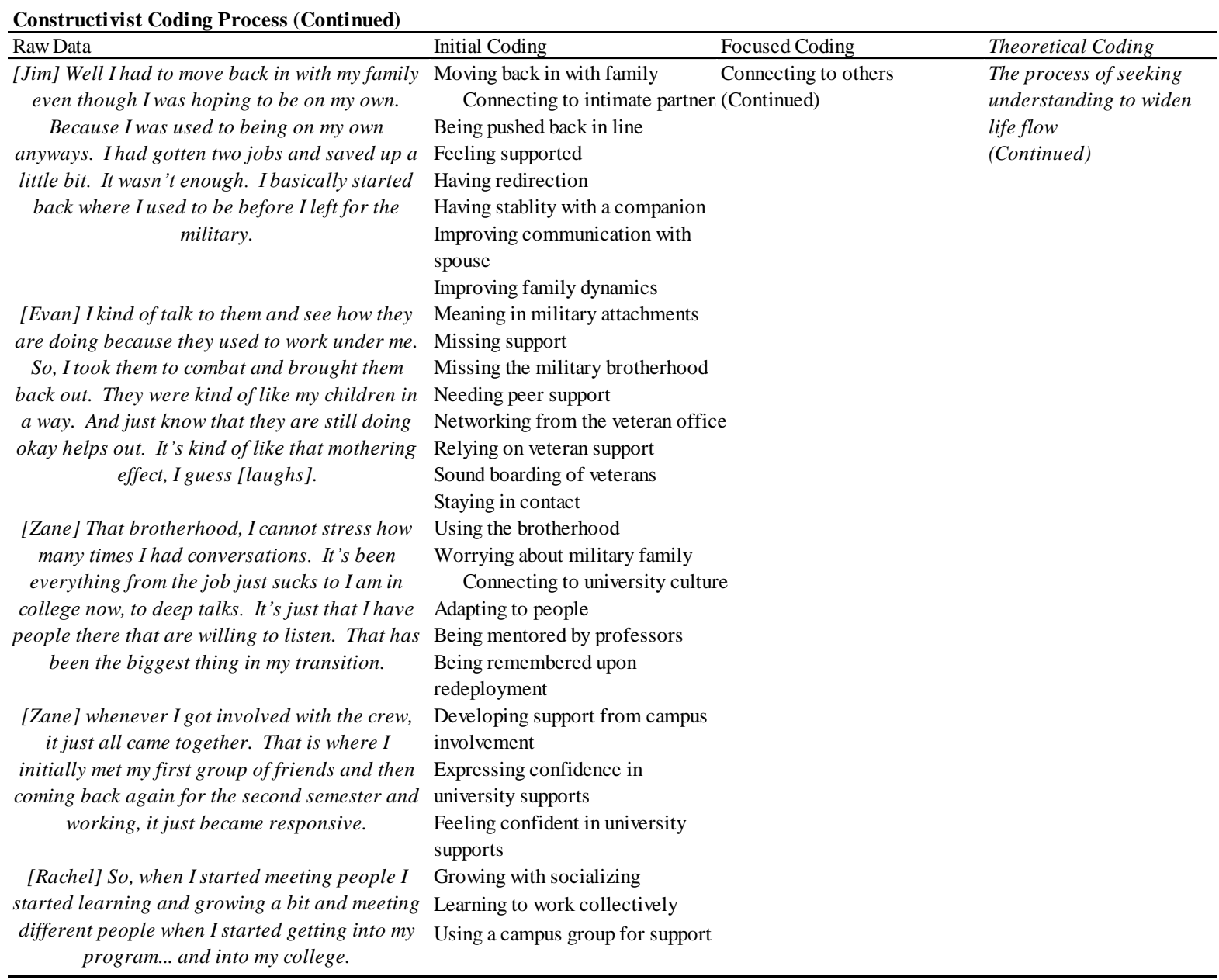




\section{APPENDIX P (continued)}

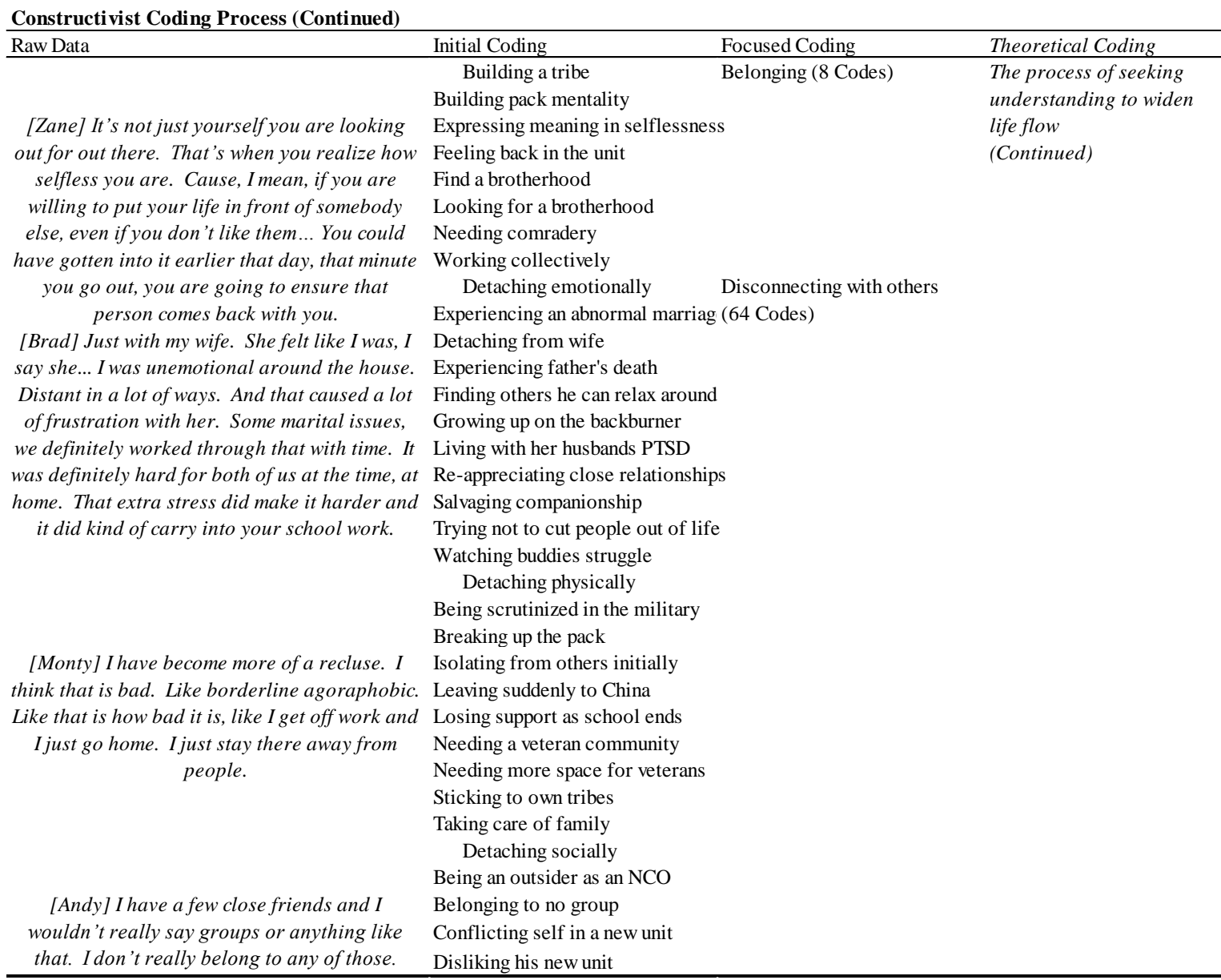




\section{APPENDIX P (continued)}

\begin{tabular}{|c|c|c|}
\hline Raw Data & Focused Coding & Theoretical Coding \\
\hline [Ron] After this summer of dealing with young & Desiring not to connect with stude Disconnecting with others & The process of seeking \\
\hline kids isolated on a study abroad program, ah, I & Disliking new social interactions (Continued) & understanding to widen \\
\hline mean there was a lot of unnecessary drama... & Feeling a disconnect & life flow \\
\hline And things that don't need to exist in life. I & Feeling alienated & (Continued) \\
\hline think because you know, they are young and & Feeling unconnected with classmates & \\
\hline they are dumb that... I don't have a desire to & Giving direction & \\
\hline connect with them. & Inability to relate & \\
\hline [Rachel] According to my friends, people who & Intimidating others & \\
\hline have been with me since like undergrad, they & Lacking common interests & \\
\hline were like intimidated and like scared of me in & Losing military support & \\
\hline the beginning. But now they are like oh that is & Making friends difficult & \\
\hline just your personality. You know, kind of take & Not looking foward to college interactions & \\
\hline charge and be the ready type thing. & Not wanting to be involved & \\
\hline [Tim] I didn't feel like anyone reached out to & Reaching out to veterans & \\
\hline me or wanted me to be involved with any kind & Struggling to relate to veterans & \\
\hline of event. I felt, I was a commuter and I was a & Trying to fit in as a normal student & \\
\hline $\begin{array}{l}\text { non-traditional student. And I definitely felt as } \\
\text { such. }\end{array}$ & Wanting personal respect & \\
\hline
\end{tabular}




\section{APPENDIX P (continued)}

Constructivist Coding Process (continued)

Raw Dat

Initial Coding

Feeling supported by friends

Focused Coding

Bringing stability to military

self (22 Codes)

Theoretical Coding

Learning postive life lessons

[Shelly] It (the military) revealed things to me Learning resilience in the military that I didn't know were going on. Like health- Learning to cope with PTSD

wise. Things that I didn't know I was composed Learning to do things on your own

of... I don't know, it seems to bring out the best Learning to self-adapt

and the worst of you. If that makes any sense. Managing self

The best meaning that, I would say the best was Managing triggers

$$
\text { the resilience. }
$$

Overcoming learning disabilities

[Evan] Me being in school, there are certain Overcoming personal perceptions

moments when you have to go out to Walmart. Overcoming PTSD issues

You do have to go pick something up from the Overcoming stressors

store. There is just people there. Having to do recognizing false beliefs

those things, I have to adapt. Oh, okay, this Resolving hatred

wasn't as bad as I thought and it is a little Taking her medication

easier. By doing, I have been able to make it Using emotions other than hatred

easier.

Valuating veteran experiences

Wanting better things

Wanting something better

Building self in ways to

achieve student succes

Cultivating student veteran self

Being better prepared for

difficult classes

Being challenged by school

[Tim] I was kind of scared of challenges. I thought the next one was going to break me.

Even though I had been through so much any Being culturally sensitive Being positive

way. I was still scared, but now I really see the Affirming postive outlook

importance of difficult situations in learning. Being aware of challenges Feeling driven

[Tim] I have learned that the biggest positive Feeling mentally resilient

change is that I have embraced struggle... I Feeling safe think this is the first time in my life where have seen the value of struggle.

The process of gaining

stability to widen life flow

Flowing strong

Learning independence 


\section{APPENDIX P (continued)}

\begin{tabular}{|c|c|c|c|}
\hline Raw Data & Initial Coding & Focused Coding & Theoretical Coding \\
\hline [Zane] If I fail a class, which I am now, than I & Maintaining motivation in school & Cultivating student veteran self & The process of gaining \\
\hline just look to the positives. I have to work & Moving in a positve direction & (Continued) & stability to widen life flow \\
\hline harder, I have to study harder. I have to find & Pushing at life obstacles & & (Continued) \\
\hline something in this class that draws my attention & Understanding challenges exist & & \\
\hline that can get my military side going. & Working around struggles & & \\
\hline & Being prompt & & \\
\hline & Being satisfied & & \\
\hline [James] I have really bombed tests. And if I & Building conceptulization of & & \\
\hline just don't get it, then I go to Youtube and I find & learning materials & & \\
\hline other sources to figure it out. & Building confidence in school & & \\
\hline & Defining self & & \\
\hline [Shelly] I do understand Excel a lot better than & Developing computer skills & & \\
\hline before... I would say, basic skills like computer & Doing something to get going & & \\
\hline skills, to assume that everybody does email or & Expanding with growing & & \\
\hline this... that veteran may not have done that. & Giving back to veterans & & \\
\hline They may not have had that exposure during & Growing from experiences & & \\
\hline their time on active duty. They may have been & Having a new perspective & & \\
\hline doing other things, more hands-on work. So, I & Learning about her other self & & \\
\hline think the computer skills are very important. & Proving self-worth & & \\
\hline & Sensitizing group interactions & & \\
\hline & Taking a step-back & & \\
\hline & Using the gym to cope & & \\
\hline [Zane] And another thing that helped me was, I & Using writing to cope & & \\
\hline turned to writing. So, I like to write poetry and & Being tactful & Gaining control in school & \\
\hline music and that helps me a lot. So, I can put my & Dealing with stages in life & environment & \\
\hline feelings on a piece of paper and I don't have to & Easing into school & (25 Codes) & \\
\hline worry about it. & Feeling unstable following militar & & \\
\hline & Finding a job fast & & \\
\hline & Finding meaning in school progres & & \\
\hline my issues just like everyone else does, and & Finding ways to pay the bills & & \\
\hline even though I may not be the most optimistic & Gaining momentum for grad schor & & \\
\hline cheery person in the world, I find equality, I & Getting conflicts resolved & & \\
\hline find assurance, and I find certain kind of aspect & Having control over the paddle & & \\
\hline in my life that I am creating that I think is good. & Learning time management & & \\
\hline
\end{tabular}




\section{APPENDIX P (continued)}

Constructivist Coding Process (continued)

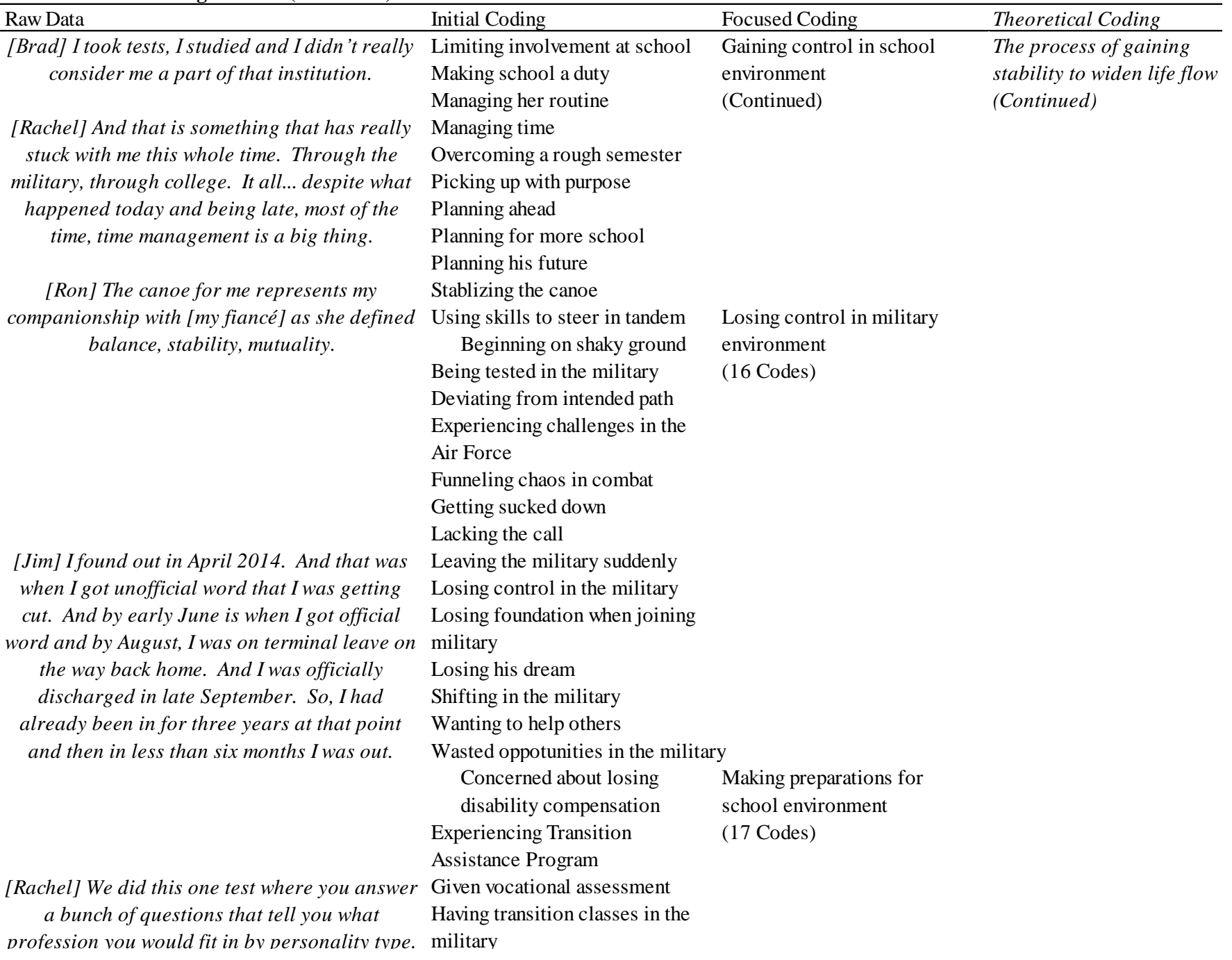




\section{APPENDIX P (continued)}

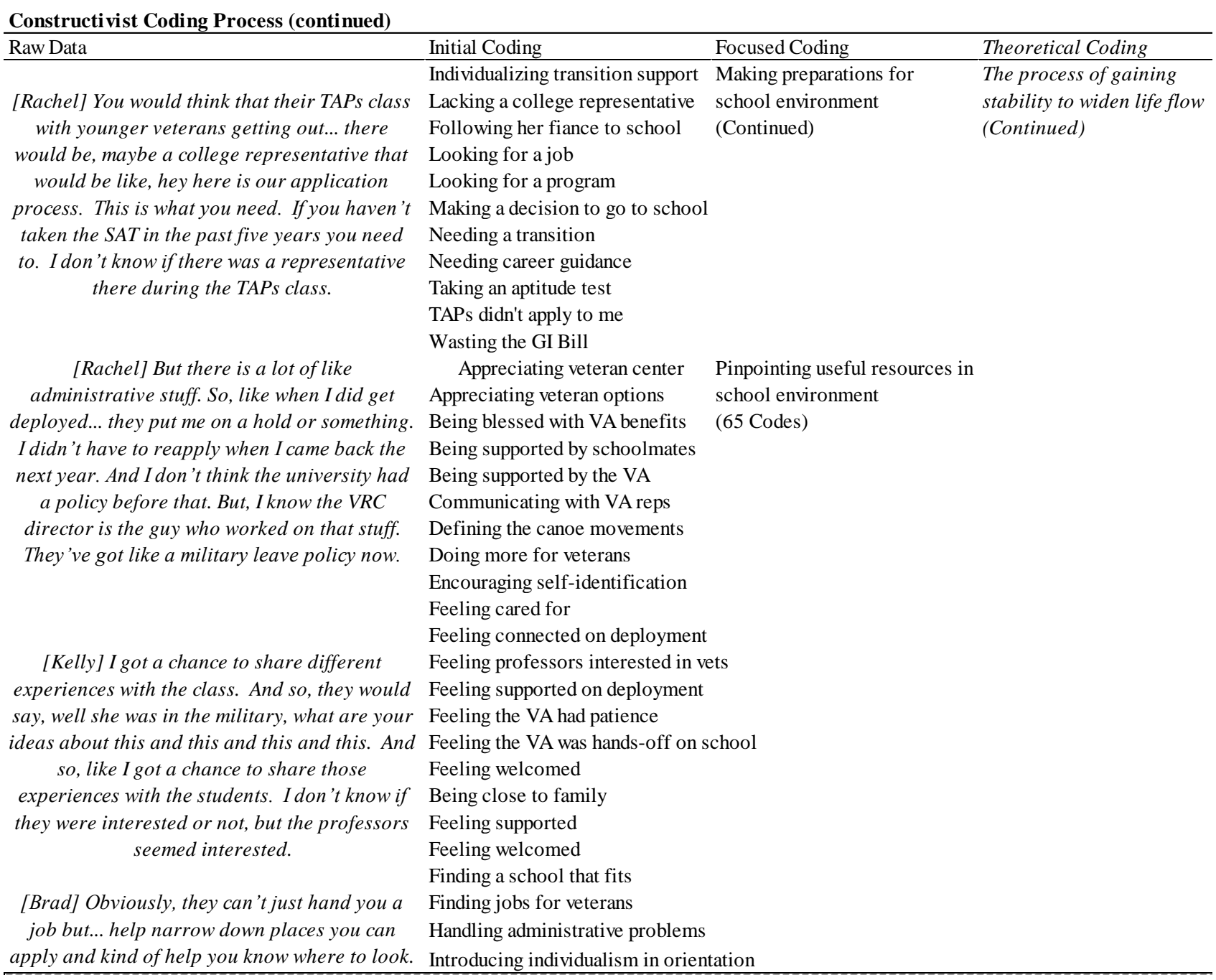




\section{APPENDIX P (continued)}

Constructivist Coding Process (continued)

\begin{tabular}{|c|c|c|c|}
\hline Raw Data & Initial Coding & Focused Coding & Theoretical Coding \\
\hline [Brad] Without the voc-rehab and the GI Bill, I & Needing extra income & Pinpointing useful resources in & The process of gaining \\
\hline would not have went to school. There is no way & Needing the GI Bill & school environment & stability to widen life flow \\
\hline that I would have been able to find any kind of & Needing the VA & (Continued) & (Continued) \\
\hline a decent job. & \multicolumn{3}{|l|}{ Opening new possibilities } \\
\hline [Tim] I think that would have helped me. I & \multicolumn{3}{|l|}{ Preferring a veteran orientation } \\
\hline came in before that (Vet Orientation) was & \multicolumn{3}{|l|}{ Preserving educational entitlement } \\
\hline mandatory for new students. And even if it & \multicolumn{3}{|l|}{ Providing an atmosphere } \\
\hline wasn't mandatory, if it was offered, I probably & \multicolumn{3}{|l|}{ Providing GI Bill education } \\
\hline wouldn't have gone to it, to be honest. Because & \multicolumn{3}{|c|}{ Providing VA health claims education } \\
\hline as a veteran I think, I got things figured out, I & \multicolumn{3}{|c|}{ Receiving a scholarship during deployment } \\
\hline am high on the horse from my military service. & \multicolumn{3}{|c|}{ Receiving helpful assistance } \\
\hline & \multicolumn{3}{|c|}{ Recieving support from the community } \\
\hline [Kelly] Like even when I started going to grad & \multicolumn{3}{|l|}{ Recognizing veterans' family needs } \\
\hline school, they said some people get divorced & \multicolumn{3}{|c|}{ Recommending veteran-centric orientations } \\
\hline when you go to school. And I am like, well I am & \multicolumn{3}{|c|}{ Recommending mandatory orientations } \\
\hline not interested in that. And what if I am already & \multicolumn{3}{|l|}{ Selecting a veteran-friendly institution } \\
\hline in this stage of my life and I have a family, how & \multicolumn{3}{|l|}{ Using campus resources } \\
\hline am I ever going to go back to school, if it & \multicolumn{3}{|l|}{ Using resources available } \\
\hline consumes that much of my time? & \multicolumn{3}{|l|}{ Using resources to get learn better } \\
\hline & \multicolumn{3}{|c|}{ Balancing control Regaining Control } \\
\hline [James] I like to finish. I will be the only & Completing goals & (24 Codes) & \\
\hline person in my family to have a bachelor's & \multicolumn{3}{|l|}{ Continuing to look } \\
\hline degree. So, all the other cousins in my family & \multicolumn{3}{|l|}{ Enjoying being out } \\
\hline have associate's degrees. So, being the best at & \multicolumn{3}{|l|}{ Enjoying independence } \\
\hline finishing, that kind of thing. & \multicolumn{3}{|l|}{ Feeling in control } \\
\hline & \multicolumn{3}{|l|}{ Figuring out what to be } \\
\hline & \multicolumn{3}{|l|}{ Figuring out what to do } \\
\hline & \multicolumn{3}{|l|}{ Figuring out what to do in life } \\
\hline $\begin{array}{l}\text { [Kelly] I just really didn t know what to do with } \\
\text { my life at that point. I couldn't go and get a }\end{array}$ & \multicolumn{3}{|l|}{ Finding a new normal } \\
\hline job. I had a newborn and my husband was & \multicolumn{3}{|l|}{ Finding purpose through spirituality } \\
\hline working eighty hours a week for us to barely & \multicolumn{3}{|l|}{ Focusing on self } \\
\hline make it in the beginning stages of all that. It & \multicolumn{3}{|l|}{ Persevering } \\
\hline was really hard and I went into a depression. & \multicolumn{3}{|l|}{ Realizing a different purpose } \\
\hline & \multicolumn{3}{|l|}{ Realizing his direction in life } \\
\hline
\end{tabular}




\section{APPENDIX P (continued)}

\section{Constructivist Coding Process (continued)}

\begin{tabular}{|c|c|c|c|}
\hline Raw Data & Initial Coding & Focused Coding & Theoretical Coding \\
\hline [Ron] I joined the military and I went through & Realizing his future & \multirow{5}{*}{$\begin{array}{l}\text { Regaining Control } \\
\text { (Continued) }\end{array}$} & The process of gaining \\
\hline infantry school... deployed to Iraq for twelve & Searching for something & & stability to widen life flow \\
\hline months and the beginnings of that deployment I & Steering your own ship & & (Continued) \\
\hline was still new to the military. I had been in less & Testing the waters & & \\
\hline than a year. I was kind of getting my feeling & Trying to find balance & & \\
\hline for things. By the end, I was done. I had & Analyzing the big picture & \multirow{4}{*}{$\begin{array}{l}\text { Using military strengths } \\
\text { ( } 21 \text { Codes) }\end{array}$} & \\
\hline realized that I did not want to do it for the rest & Being accountable & & \\
\hline of my life. Irealized that it wasn't what I was & Being accountable to mistakes & & \\
\hline meant to be doing. & Being self-reliant & & \\
\hline [Jim] I would hope that I have a little resiliency & Being resilient & \multirow[t]{4}{*}{ Being resilient } & \\
\hline since, I have went through what I went through & \multirow{3}{*}{$\begin{array}{l}\text { Being serious about school } \\
\text { Giving constant effort } \\
\text { Recognizing responsibilities }\end{array}$} & & \\
\hline \multirow[t]{2}{*}{ and it hasn't affected me too, horribly. } & & & \\
\hline & & & \\
\hline [Jim] No use sitting around and getting & \multicolumn{2}{|l|}{ Setting Goals } & \\
\hline depressed about it. You learn from that and & \multicolumn{2}{|l|}{ Wanting to be prepared } & \\
\hline you move on. And that is what I am trying to & \multicolumn{2}{|c|}{ Avoiding trial and error } & \\
\hline do. The fact that I, one big thing that I am good & \multicolumn{2}{|c|}{ Feeling unprepared for responsiblities } & \\
\hline at, I am good at setting goals. & \multicolumn{2}{|c|}{ Knowing information concretely } & \\
\hline & \multicolumn{2}{|l|}{ Needing visual examples } & \\
\hline [James] I was in class and I pulled out my & \multicolumn{2}{|l|}{ Staying organized } & \\
\hline folder and it was all organized and this frat- & \multicolumn{2}{|l|}{ Visual learning from the military } & \\
\hline $\begin{array}{l}\text { boy saw it and mentioned, wow you are so } \\
\text { organized. And I said, well it would help you }\end{array}$ & \multicolumn{2}{|l|}{ Working hard in class } & \\
\hline out too, if you were this organized. And I can't & & & \\
\hline seem to grasp when these kids seem to pull out & & & \\
\hline $\begin{array}{l}\text { this wrinkled paper from their bag and turn it } \\
\qquad \text { in. }\end{array}$ & & & \\
\hline
\end{tabular}




\section{REFERENCES}

Ackerman, R., DiRamio, D., Mitchell, R.L. (2009). Transitions: combat veterans as college students. Wiley Interscience. 126. 5-14. http://dx.doi.org/10.1002/ss.311

Afari, N., Pittman, J., Floto, E., Owen, L., Buttner, M., Hossain, N., ... \& Lohr, J. B. (2015). Differential impact of combat on postdeployment symptoms in female and male veterans of Iraq and Afghanistan. Military Medicine, 180(3), 296-303.

Ahern, J., Worthen, M., Masters, J., Lippman, S. A., Ozer, E. J., \& Moos, R. (2015). The Challenges of Afghanistan and Iraq Veterans' Transition from Military to Civilian Life and Approaches to Reconnection. PloS One, 10(7), 1-13. http://dx.doi.org/10.1371/journal.pone.0128599

Aldwin, C., \& Levenson, M. (2005). Commentary: Military service and emotional maturation: The Chelsea pensioners. New York, NY: Springer.

Aldwin, C., Levenson, M., \& Spiro, A. (1994). Vulnerability and resilience to combat exposure: Can stress have lifelong effects? Psychology and Aging, 9, 34-44. http://dx.doi.org/10.1037/0882-7974.9.1.34.

American Council on Education, Center for Policy Research and Strategy. (2014). Higher ed spotlight: Undergraduate student veterans. (Infographic). Retrieved from http://www.acenet.edu/news-room/Documents/Higher-ed-spotlightundergraduate-student-veterans.pdf

American Occupational Therapy Association. (2007). AOTA's Centennial Vision and executive summary. American Journal of Occupational Therapy, 61, 613-614. http://dx.doi.org/10.5014/ajot.61.6.613 
American Occupational Therapy Association. (2010). Specialized knowledge and skills in mental health promotion, prevention, and intervention in occupational therapy practice. American Journal of Occupational Therapy, 64(Suppl.), S30-S43. http://dx.doi.org/10.5014/ajot.2010.64S30-64S43

American Occupational Therapy Association (AOTA). (2013). Societal statement on occupational therapy in the promotion of health and well-being. American Journal of Occupational Therapy, 67.

American Occupational Therapy Association. (2014). Occupational therapy practice framework: Domain and process (3rd ed.). American Journal of Occupational Therapy, 68(Suppl.1), S1-S48. http://dx.doi.org/10.5014/ajot.2014.682006

American Psychiatric Association. (2013). Diagnostic and Statistical Manual of Mental Disorders (DSM-5®). American Psychiatric Pub.

Ames, G. M., Cunradi, C. B., Moore, R. S., \& Stern, P. (2007). Military culture and drinking behavior among US Navy careerists. Journal of studies on alcohol and drugs, 68(3), 336-344.

Anderson, M., Goodman, J., \& Schlossberg, N. K. (2011). Counseling adults in transition: Linking Schlossberg's theory with practice in a diverse world. New York, NY: Springer.

Barnard-Brak, L., Bagby, J., Jones, J., \& Sulak, T. (2011). Teaching post 9/11 studentveterans with symptoms of PTSD: The influence of faculty perceptions and selfefficacy. Journal of Vocational Rehabilitation. 35, 29-36. 
Beck, C. E., Gonzales, F., Sells, C. H., Jones, C., Reer, T., \& Zhu, Y. (2012). The effects of animal-assisted therapy on wounded warriors in an occupational therapy life skills program. US Army Medical Department Journal, 38-45.

Belanger, H. G., Uomoto, J. M., \& Vanderploeg, R. D. (2009). The Veterans Health Administration system of care for mild traumatic brain injury: Costs, benefits, and controversies. The Journal of head trauma rehabilitation, 24(1), 4-13.

Bellotti, C., Laffaye, C., Weingardt, K. R., Fischer, M., \& Schumacher, T. (2011). Revisioning veteran readjustment: Evaluating outcomes of a green-jobs training program. Journal of Vocational Rehabilitation, 35(1). 51-57. http://dx.doi.org/10.3233/JVR-2011-553

Betancourt, T., Borisova, I., Williams, T. P., Meyers-Ohki, S. E., Rubin-Smith, J. E., Annan, J., \& Kohrt, J. A. (2013). Research Review: Psychosocial adjustment and mental health in former child soldiers - a systematic review of the literature and recommendations for future research. Journal of Child Psychology \& Psychiatry, 54(1), 17-36. http://dx.doi.org/10.1111/j.1469-7610.2012.02620.x

Bowles, S. V., \& Bates, M. J. (2010). Military organizations and programs contributing to resilience building. Military Medicine, 175(6), 382-385. http://dx.doi.org/10.7205/milmed-d-10-00099

Braun, V. \& Clarke, V. (2006). Using thematic analysis in psychology. Qualitative Research in Psychology, 3(2), 77-101.

Bray, R. M., Fairbank, J. A., \& Marsden, M. E. (1999). Stress and substance use among military women and men. The American Journal of Drug and Alcohol Abuse, 25(2), 239-256. http://dx.doi.org/10.1081/ada-100101858 
Bridges, W. (2004). Transitions: Making sense of life's changes. Cambridge, MA: Da Capo Press.

Brown, W. B. (2011). From war zones to jail: Veteran reintegration problems. JUST. POL'Y J., 8, 1-30.

Bryant, A., \& Charmaz, K. (Eds.). (2007). The sage handbook of grounded theory. Sage.

Bullock, E. E., Braud, J., Andrews, L., \& Phillips, J. (2009). Career concerns of unemployed U.S. war veterans: Suggestions from a cognitive information processing approach. Journal of Employment Counseling, 46(4), 171-181.

Burnett-Zeigler, I., Ilgen, M., Valenstein, M., Zivin, K., Gorman, L., Blow, A., ... \& Chermack, S. (2011). Prevalence and correlates of alcohol misuse among returning Afghanistan and Iraq veterans. Addictive Behaviors, 36(8), 801-806.

Burnett-Zeigler, I., Valenstein, M., Ilgen, M., Blow, A. J., Gorman, L. A., \& Zivin, K. (2011). Civilian employment among recently returning Afghanistan and Iraq National Guard veterans. Military Medicine, 176(6), 639-646.

Bush, N. E., Reger, M. A., Luxton, D. D., Skopp, N. A., Kinn, J., Smolenski, D., \& Gahm, G. A. (2013). Suicides and suicide attempts in the US military, 20082010. Suicide and Life-Threatening Behavior, 43(3), 262-273.

Caffrey, R. J. (2009). Substance abuse and veterans. Retrieved from http://www.caffreycounseling.com/SubstanceAbuseinVeterans.html

Carmody, S., Nolan, R., Ni Chonchuir, N., Curry, M., Halligan, C., \& Robinson, K. (2007). The guiding nature of the kawa (river) model in Ireland: creating both opportunities and challenges for occupational therapists. Occupational Therapy International, 14(4), 221-236. 
Carter-Pokras, O., \& Bethune, L. (2009). Defining and measuring acculturation:

a systematic review of public health studies with Hispanic populations in the United States. A commentary on Thomson and Hoffman-Goetz. Social Science \& Medicine, 69(7), 992-995.

Casella, L., \& Motta, R. (1990). Comparison of characteristics of Vietnam veterans with and without Posttraumatic Stress Disorder. Psychological Reports, 67, 595-605. http://dx.doi.org/10.2466/PR0.67.6.595-605

Cate, C. A. (2014). Million records project: Research from student veterans of America. Washington, DC: Student Veterans of America.

Charmaz, K. (2006). Constructing grounded theory: A practical guide through qualitative research. London: Sage.

Charmaz, K. (2008). Grounded theory as an emergent method. In S. N. Hesse-Biber \& P. Leavy (Eds), Handbook of Emergent Methods (pp. 155-172). New York: The Guilford Press.

Charmaz, K. (2011). Grounded theory methods in social justice research. Thousand Oaks, CA: Sage.

Charmaz, K. (2014). Constructing grounded theory. ( $2^{\text {nd }}$ ed.). London: Sage.

Charmaz, K. (2015). Teaching theory construction with initial grounded theory tools: A Reflection on lessons and learning. Qualitative Health Research, 25(12), 16101622. http://dx.doi.org/10.1177/1049732315613982

Church, T. E. (2009). Returning Veterans on Campus with War Related Injuries and the Long Road Back Home. Journal of Postsecondary Education and Disability, 22(1), 43-52. 
Clark, M. E., Bair, M. J., Buckenmaier, C. C., III, Gironda, R. J., \& Walker, R. L. (2007). Pain and combat injuries in soldiers returning from Operations Enduring Freedom and Iraqi Freedom: Implications for research and practice. Journal of Rehabilitation Research and Development, 44, 179-194.

Clark, M. E., Scholten, J. D., Walker, R. L., \& Gironda, R. J. (2009). Assessment and Treatment of Pain Associated with Combat-Related Polytrauma. Pain Medicine, 10(3), 456-469.

Clark, M. E., Walker, R. L., Gironda, R. J., \& Scholten, J. D. (2009). Comparison of pain and emotional symptoms in soldiers with polytrauma: unique aspects of blast exposure. Pain Medicine, 10(3), 447-455.

Classen, S., Monahan, M., Canonizado, M., \& Winter, S. (2014). Utility of an occupational therapy driving intervention for a combat veteran. American Journal of Occupational Therapy, 68, 405-411. http://dx.doi.org/10.5014/ajot.2014.010041

Clemens, E. V., \& Milsom, A. S. (2008). Enlisted Service Members' Transition into the Civilian World of Work: A Cognitive Information Processing Approach. Career Development Quarterly, 56(3), 246-256.

Cogan, A. M. (2014). The Issue Is-Supporting our military families: A case for a larger role for occupational therapy in prevention and mental health care. American Journal of Occupational Therapy, 68, 478-483. http://dx.doi.org/10.5014/ajot.2014.009712

Cook, B. J., \& Kim, Y. (2009). From soldier to student: Easing the transition of service Members on Campus. Washington, DC: American Council on Education. 
Cornum, R., Matthews, M. D., \& Seligman, M. E. (2011). Comprehensive soldier fitness: building resilience in a challenging institutional context. American Psychologist, 66(1), 4.

Creswell, J. W. (2013). Qualitative inquiry and research design: Choosing among five approaches. Sage.

Daly, D. D., \& Garrity, B. K. F. (2013). From boots on the ground to seats in the classroom: An assessment of institutional structure and veteran students. American Journal of Educational Studies, 6(1). 5-17.

Davidson, R. J., Kabat-Zinn, J., Schumacher, J., Rosenkranz, M., Muller, D., Santorelli, S. F., ... Sheridan, J. F. (2003). Alterations in brain and immune function produced by mindfulness meditation. Psychosomatic Medicine, 65(4), 564-570.

Demers, A. (2011). When veterans return: The role of community in reintegration. Journal of Loss and Trauma, 16(2), 160-179.

Department of Veterans Affairs Epidemiology Program. (2011). Analysis of VA health care utilization among Operation Enduring Freedom (OEF), Operation Iraqi Freedom (OIF), and Operation New Dawn (OND) veterans. Post Deployment Health Strategic Healthcare Group, Office of Public Health, Quarter 4.

Dillman, D. A., Smyth, J. D., \& Christian, L. M. (2009). Internet, mail, and mixed-mode questionnaires: The tailored design method. Hoboken, NJ: John Wiley \& Sons DiRamio, D., Ackerman, R., \& Garza-Mitchell, R. L. (2008). From combat to campus: Voices of student-veterans. NASPA Journal, 45(1), 73-102. http://dx.doi.org/10.2202/1949-6605.1908 
DiRamio D \& Spires, M. (2009). Partnering to assist disabled veterans in transition. Wiley Interscience. 126, 81-88. http://dx.doi.org/10.1002/ss.319

Durdella, N., \& Kim, Y. K. (2012). Understanding patterns of college outcomes among student veterans. Journal of Studies in Education, 2(2), 109-129.

Eaker, S., Bergström, R., Bergstrom, A., Adami, H. O., \& Nyren, O. (1998). Response rate to mailed epidemiologic questionnaires: A population-based randomised trial of variations in design and mailing routines. American Journal of Epidemiology, 147, 74-82. http://dx.doi.org/10.1093/725oxfordjournals.aje.a009370

Eakman, A. M., Schelly, C., \& Henry, K. L. (2016). Protective and vulnerability factors contributing to resilience in post-9/11 veterans with service-related injuries in postsecondary education. American Journal of Occupational Therapy, 70, 7001260010. http://dx.doi.org/10.5014/ajot.2016.016519

Eaton, K., Hoge, C., Messer, S., Whitt, A., Cabrera, O., McGurk, D., \& ... Castro, C. (2008). Prevalence of mental health problems, treatment need, and barriers to care among primary care-seeking Spouses of military service members involved in Iraq and Afghanistan deployments. Military Medicine, 173(11), 1051-1056.

Elbogen, E. B., Johnson, S. C., Wagner, H. R., Newton, V. M., \& Beckham, J. C. (2012). Financial well-being and postdeployment adjustment among Iraq and Afghanistan war veterans. Military Medicine, 177(6), 669-675.

Elder, G., \& Clipp, E. (1988). Combat experience, comradeship, and psychological health. In Human adaptation to extreme stress: From the Holocaust to Vietnam (pp. 131-156). New York, NY: Plenum. 
Elliott, M., Gonzalez, C., \& Larsen, B. (2011). U.S. military veterans transition to college: Combat, PTSD, and alienation on campus. Journal of Student Affairs Research and Practice, 48(3), 279-296. http://dx.doi.org/10.2202/1949-6605.6293.

Ellison, M. L., Mueller, L., Smelson, D., Corrigan, P. W., Stone, R. A. T., Bokhour, B. G., ... \& Drebing, C. (2012). Supporting the education goals of post-9/11 veterans with self-reported PTSD symptoms: A needs assessment. Psychiatric Rehabilitation Journal, 35(3), 209-217. http://dx.doi.org/10.2975/35.3.2012.209.217

Ellsworth, P.D., Sinnott, M.W., Laedtke, M.E., McPhee, S.D. (1993). Utilization of occupational therapy in combat stress control during the Persian Gulf War. Military Medicine, 158(6), 381-385.

Erbes, C. R., Kaler, M. E., Schult, T., Polusny, M. A., \& Arbisi, P. A. (2011). Mental health diagnosis and occupational functioning in National Guard/Reserve veterans returning from Iraq. Journal of Rehabilitation Research and Development, 48(10), 1159-1170.

Erickson, M. W., Brininger, T. L., Newton, S. M., Mattila, A. M., \& Burns, J. P. (2011). US Army occupational therapy: Promoting optimal performance. In E. Ritchie (Ed.), Combat and Operational Behavioral Health (pp. 357-374). Washington, DC: Borden Institute.

Esterling, B. A., L’Abate, L., Murray, E. J., \& Pennebaker, J. W. (1999). Empirical foundations for writing in prevention and psychotherapy: Mental and physical 
health outcomes. Clinical Psychology Review, 19(1), 79-96.

http://dx.doi.org/10.1016/s0272-7358(98)00015-4

Faulkner, R. R., \& McGaw, D. B. (1977). Uneasy Homecoming:" Stages in the Reentry Transition of Vietnam Veterans". Journal of Contemporary Ethnography, 6(3), 303.

Fisher, A. G., \& Griswold, L. A. (2010). Evaluation of Social Interaction (2nd ed.). Ft. Collins, CO: Three Star Press.

Ford, D., Northrup, P., \& Wiley, L. (2009). Connections, partnerships, opportunities, and programs to enhance success for military students. New Directions for Student Services, 126, 61-69.

Gawande, A. (2004). Casualties of war-military care for the wounded from Iraq and Afghanistan. New England Journal of Medicine, 351(24), 2471-2475.

Gergen, K. J. (2001b). Social construction in context. Thousand Oaks, CA: Sage.

Gerardi, S. M. (1999). Part I. Work hardening for warriors: Occupational therapy for combat stress casualties. Work: A Journal of Prevention, Assessment and Rehabilitation, 13(3), 185-195.

Gibson, R. W., D’Amico, M., Jaffe, L., \& Arbesman, M. (2011). Occupational therapy interventions for recovery in the areas of community integration and normative life roles for adults with serious mental illness: A systematic review. American Journal of Occupational Therapy, 65, 247-256.

http://dx.doi.org/10.5014/ajot.2011.001297

Gironda, R. J., Clark, M. E., Ruff, R. L., Chait, S., Craine, M., Walker, R., \& Scholten, J. (2009). Traumatic brain injury, polytrauma, and pain: challenges and treatment 
strategies for the polytrauma rehabilitation. Rehabilitation psychology, 54(3), 247-258. http://psycnet.apa.org/doi/10.1037/a0016906

Gregg, B.T., Howell, D.M., Quick, C.D., \& Iwama, M.K. (2015). The Kawa River Model: Applying Theory to Develop Interventions for Combat and Operational Stress Control. Occupational Therapy in Mental Health, 31(4), 366-384.

Griffin, K. A., \& Gilbert, C. K. (2015). Better transitions for troops: An application of Schlossberg's transition framework to analyses of barriers and institutional support structures for student veterans. Journal of Higher Education, 86(1), 7197. http://dx.doi.org/10.1353/jhe.2015.0004

Grossman, P., Niemann, L., Schmidt, S., \& Walach, H. (2004). Mindfulness-based stress reduction and health benefits: A meta-analysis. Journal of Psychosomatic Research, 57(1), 35-43.

Haynie, J. M., \& Shepherd, D. (2011). Toward a theory of discontinuous career transition: investigating career transitions necessitated by traumatic life events. Journal of Applied Psychology, 96(3), 501-524. http://psycnet.apa.org/doi/10.1037/a0021450

Higate, P. R. (2001). Theorizing continuity: from military to civilian life. Armed Forces \& Society, 27(3), 443-460.

Hinojosa, R., Hinojosa, M. S., Nelson, K., \& Nelson, D. (2010). Veteran family reintegration, primary care needs, and the benefit of the patient-centered medical home model. The Journal of the American Board of Family Medicine, 23(6), 770774. 
Hoge, C. W., Castro, C. A., Messer, S. C., McGurk, D., Cotting, D. I., \& Koffman, R. L. (2004). Combat duty in Iraq and Afghanistan, mental health problems, and barriers to care. New England Journal of Medicine, 351, 13-22.

\section{http://dx.doi.org/10.1056/NEJMoa040603}

Hoge, C. W., Auchterlonie, J. L., \& Milliken, C. S. (2006). Mental health problems, use of mental health services, and attrition from military service after returning from deployment to Iraq or Afghanistan. Journal of the American Medical Association, 295(9), 1023-1032. http://dx.doi.org/10.1001/jama.295.9.1023

Hoge, C. W., Terhakopian, A., Castro, C. A., Messer, S. C., \& Engel, C. C. (2007). Association of posttraumatic stress disorder with somatic symptoms, health care visits, and absenteeism among Iraq war veterans. American Journal of Psychiatry, 164, 150-153. http://dx.doi.org/10.1176/appi.ajp.164.1.150

Holland, J. L., Messer, M. A., \& PAR Staff. (2013). Self-Directed Search (SDS) Occupations Finder (5th ed.). Lutz, FL: PAR.

Holloway, K. M. (2009). Understanding reentry of the modern-day student-veteran through Vietnam-era theory. Journal of Student Affairs, 18, 11-17.

Hood, J. C. (2007). Orthodoxy vs. power: The defining traits of grounded theory. The sage handbook of grounded theory, 151-164.

Hotchkiss, M. (2012). 2010 ACS Content Test Evaluation Report Covering Veteran Status (Final Report: ACS12-RER-07). American Community Survey Research and Evaluation Program. Washington, DC: U.S. Census Bureau. Retrieved from: http://www.census.gov/acs/www/Downloads/library/2012/2012_Hotchkiss_02.pd f. 
Humbert, T. K., Bess, J. L., \& Mowery, A. M. (2013). Exploring women’s perspectives of overcoming intimate partner violence: A phenomenological study. Occupational Therapy in Mental Health, 29(3), 246-265.

\section{http://dx.doi.org/10.1080=0164212x.2013.819465}

Hunt, L. M. L. M., Schneider, S., \& Comer, B. (2004). Should “acculturation" be a variable in health research? A critical review of research on US Hispanics. Social Science \& Medicine, 59(5), 973-986.

IOM (Institute of Medicine). 2013. Returning home from Iraq and Afghanistan: Assessment of readjustment needs of veterans, service members, and their families. Washington, DC: The National Academies Press.

Iversen, A., Liddell, K., Fear, N., Hotopf, M., \& Wessely, S. (2006). Consent, confidentiality, and the data protection act. British Medical Journal, 332, 165169. http://dx.doi.org/10.1136/bmj.332.7534.165

Iwama, M. (2005). The Kawa (river) model: Nature, life flow, and the power of culturally relevant occupational therapy. In F. Kronenberg, S. Algado, \& N. Pollard (Eds.), Occupational therapy without border: Learning from the spirit of survivors (pp. 213-227). Churchill Livingston: Elsevier.

Iwama, M. (2007). Culture and occupational therapy: Meeting the challenge of relevance in a global world. Occupational Therapy International, 14(4), 183-187. http://dx.doi.org/10.1002=oti.234

Iwama, M., Farhan, H., Hanrahan, E., Kaufman, A., Lim, K. H., \& Patel, N. (2011). The Kawa (river) model: Culturally responsive occupational therapy without borders. In F. Kronenberg, N. Pollard, \& S. Dikaios (Eds.), Occupational 
therapies without borders-volume 2: Towards an ecology of occupation-based practices (pp. 257-266). Edinburgh: Elsevier.

Iwama, M. K. (2006). The Kawa model: Culturally relevant occupational therapy. Churchill Livingston: Elsevier.

Iwama, M. K., Thomson, N. A., Macdonald, \& R. M. (2009). The Kawa model: The power of culturally responsive occupational therapy. Disability \& Rehabilitation, 31(14), 1125-1135. http://dx.doi.org/10.1080=09638280902773711

Jacobson, I. G., Ryan, M. A., Hooper, T. I., Smith, T. C., Amoroso, P. J., Boyko, E. J., ... Bell, N. S. (2008). Alcohol use and alcohol-related problems before and after military combat deployment. Journal of the American Medical Association, 300(6), 663-675. http://dx.doi.org/10.1001/jama.300.6.663

Jarrett, T. A. (2007). Warrior resilience training in operation Iraqi freedom: Combining rational emotive behavior therapy, resiliency, and positive psychology. US Army Medical Department Journal, 8(7/8/9), 32-38.

Jarrett, T. A. (2013). Warrior resilience and thriving (WRT): Rational emotive behavior therapy (REBT) as a resiliency and thriving foundation to prepare warriors and their families for combat deployment and posttraumatic growth in Operation Iraqi Freedom, 2005-2009. Journal of Rational-Emotive \& Cognitive-Behavior Therapy, 31(2), 93-107. http://dx.doi.org/10.1007/s10942-013-0163-2

Jones, K. C. (2013). Understanding student veterans in transition. The Qualitative Report, 18(74), 1-14. 
Kabat-Zinn, J. (1994). Wherever you go, there you are: mindfulness meditation in everyday life. New York, NY: Hyperion.

Kabat-Zinn, J. (2003). Mindfulness: The heart of rehabilitation. In Leskowitz, E. (Ed.), Complementary and alternative medicine in rehabilitation (pp. 11-15). Saint Louis: Churchill Livingstone.

Kabat-Zinn, J., \& Chapman-Waldrop, A. (1988). Compliance with an outpatient stress reduction program: Rates and predictors of completion. Journal of Behavioral Medicine, 11, 333-352. http://dx.doi.org/10.1007/bf00844934

Kaplan, K., \& Kielhofner, G. (1989). Occupational case analysis interview and rating scale. Thorofare, NJ: Slack Incorporated.

Kao, H. S., Hsu, M., \& Clark, L. (2004). Conceptualizing and critiquing culture in health research. Journal of Transcultural Nursing, 15(4), 269-277.

Kelty, R., Kleykamp, M., \& Segal, D. R. (2010). The Military and the Transition to Adulthood. Future of Children, 20(1), 181-207.

Kemp, J., \& Bossarte, R. (2013). Suicide data report: 2012. Department of Veterans Affairs, Mental Health Services, Suicide Prevention Program.

Koenig, C. J., Maguen, S., Monroy, J. D., Mayott, L., \& Seal, K. H. (2014). Facilitating culture-centered communication between health care providers and veterans transitioning from military deployment to civilian life. Patient Education and Counseling, 95(3), 414-420. http://dx.doi.org/10.1016/j.pec.2014.03.016

Larner, B., \& Blow, A. (2011). A model of meaning-making coping and growth in combat veterans. Review of General Psychology, 15(3), 187-197. http://dx.doi.org/10.1037/a0024810 
Larson, G. E., \& Norman, S. B. (2014). Prospective prediction of functional difficulties among recently separated veterans. Journal of Rehabilitation Research and Development, 51(3), 415-427. http://dx.doi.org/10.1682/JRRD.2013.06.0135

Law, M., Baptiste, S., Carswell, A., McColl, M. A., Polatajko, H., \& Pollack, N. (2005). Canadian Occupational Performance Measure (4th ed.). Ottawa, Ontario: CAOT Publications ACE.

Law, M., Baptiste, S., McColl, M., Opzoomer, A., Polatajko, H., \& Pollock, N. (1990). The Canadian occupational performance measure: An outcome measure for occupational therapy. Canadian Journal of Occupational Therapy, 57(2), 82-87. http://dx.doi.org/10.1177/000841749005700207

Lazarus, R. S., \& Folkman, S. (1984). Stress, coping and adaptation. New York, NY: Springer.

Lew, H. L., Otis, J. D., Tun, C., Kerns, R. D., Clark, M. E., \& Cifu, D. X. (2009). Prevalence of chronic pain, posttraumatic stress disorder, and persistent postconcussive symptoms in OIF/OEF veterans: Polytrauma clinical triad. $J$ Rehabil Res Dev, 46(6), 697-702.

Lincoln, Y.S. \& Guba, E.G. (1989). Fourth generation evaluation. Sage.

Lopata, C., Nida, R. E., \& Marable, M. A. (2006). Progressive muscle relaxation: Preventing aggression in students with EBD. Teaching Exceptional Children, $38(4), 20-25$.

Lundahl, B. W., Kunz, C., Brownell, C., Tollefson, D., \& Burke, B. L. (2010). A metaanalysis of motivational interviewing: Twenty-five years of empirical studies. 
Research on Social Work Practice, 20, 137-160.

http://dx.doi.org/10.1177/1049731509347850

Lundquist, C., Spalding, R. J., \& Landrum, R. E. (2002). College student's thoughts about leaving the university: The impact of faculty attitudes and behaviors. Journal of College Student Retention: Research, Theory and Practice, 4(2), 123-133.

Lynn, M. R. (1986). Determination and quantification of content validity. Nursing Research, 35, 382-386. http://dx.doi.org/10.1097/00006199-198611000-00017

MacLean, M. B., Van Til, L., Thompson, J. M., Sweet, J., Poirier, A., Sudom, K., \& Pedlar, D. J. (2014). Postmilitary adjustment to civilian life: Potential risks and protective factors. Physical Therapy, 94(8), 1186-1195.

http://dx.doi.org/10.2522/ptj.20120107

Mattila, A. M., Crandall, B. D., \& Goldman, S. B. (2011). U.S. Army combat operational stress control throughout the deployment cycle: A case study. Work, 38(1), 1318. http://dx.doi.org/10.3233/WOR-2011-1100

Matuska, K., \& Christiansen, C. (2008). A proposed model of lifestyle balance. Journal of Occupational Science. 15(1), 9-19.

Matuska, K. (2012). Development of the Life Balance Inventory. Occupational Therapy Journal of Research, 32(1), 220-228.

McBain, L., Kim, Y. M., Cook, B. J., \& Snead, K. M. (2012). From soldier to student II: Assessing campus programs for veterans and service members. Washington, D.C: American Council on Education. 
Military Onesource (2012). Demographics: Profile of the military community. Retrieved from http://www.militaryonesource.mil/12038/MOS/Reports/2012Demographics-Report.pdf

Mills, J., Bonner, A., \& Francis, K. (2008). The development of constructivist grounded theory. International journal of qualitative methods, 5(1), 25-35.

Montz, R., Gonzales, F. J., Bash, D., \& Carney, A. (2008). Occupational therapy role on the battlefield: An overview of combat and operational stress and upper extremity rehabilitation. Journal of Hand Therapy, 21(2), 130-136.

Morin, R. (2011). The difficult transition from military to civilian life. Pew Research Center. Retrieved from http://www.pewsocialtrends.org/files/2011/12/TheDifficult-Transition-from-Military-to-Civilian-Life.pdf

Morse, J. M. (2010). Sampling in grounded theory. The Sage handbook of grounded theory, 229-244.

Munoz, J. P. (2007). Culturally responsive caring in occupational therapy. Occupational Therapy International, 14(4), 256-280. http://dx.doi.org/10.1002=oti.238

Nahab, F. B., Kundu, P., Gallea, C., Kakareka, J., Pursley, R., Pohida, T., . . Hallett, M. (2011). The neural processes underlying self-agency. Cerebral Cortex, 21(1), 48-55. http://dx.doi.org/10.1093=cercor=bhq059

Nayback, A. M. (2008). Health disparities in military veterans with PTSD: Influential sociocultural factors. Journal of Psychosocial Nursing and Mental Health Services, 46(6), 42-51.

Nulty, D. D. (2008). The adequacy of response rates to online and paper surveys: What can be done? Assessment \& Evaluation in Higher Education, 33(3), 301-314. http://dx.doi.org/10.1080/02602930701293231 
Olsen, T., Badger, K., \& McCuddy, M. D. (2014). Understanding the student veterans' college experience: An exploratory study. U.S. Army Medical Department Journal, 8(7), 101-108.

Ouimette, P., Vogt, D., Wade, M., Tirone, V., Greenbaum, M. A., Kimerling, R., ... \& Rosen, C. S. (2011). Perceived barriers to care among veterans health administration patients with posttraumatic stress disorder. Psychological Services, 8(3), 212-223.

Parkinson, S., Forsyth, K., \& Kielhofner, G. (2004). A user's manual for the model of human occupation screening tool (MOHOST). Chicago: University of Illinois.

Patton, M. Q. (2002). Qualitative research and evaluation methods (3rd ed.). Thousand Oaks, CA: Sage.

Paxson, D., Winston, K., Tobey, T., Johnston, S., \& Iwama, M. (2012). The Kawa model: Therapists' experiences in mental health practice. Occupational Therapy in Mental Health, 28(4), 340-355.

\section{http://dx.doi.org/10.1080=0164212x.2012.708586}

Penley, J. A., Tomaka, J., \& Wiebe, J. S. (2002). The association of coping to physical and psychological health outcomes: A meta-analytic review. Journal of Behavioral Medicine, 25(6), 551-603.

Pierce, D. E. (Ed.). (2014). Occupational science for occupational therapy. Thorofare, NJ: Slack.

Pietrzak, R. H., Johnson, D. C., Goldstein, M. B., Malley, J. C., \& Southwick, S. M. (2009). Posttraumatic stress disorder mediates the relationship between mild traumatic brain injury and health and psychosocial functioning in veterans of 
Operations Enduring Freedom and Iraqi Freedom. The Journal of nervous and mental disease, 197(10), 748-753.

Plach, H. L., \& Sells, C. H. (2013). Occupational performance needs of young veterans. American Journal of Occupational Therapy, 67, 73-81. http://dx.doi.org/10.5014/ajot.2013.003871

Polkinghorne, D. E. (1989). Phenomenological research methods. In R. S. Valle \& S. Halling (Eds.), Existential-Phenomenological perspectives in psychology: Exploring the breadth of human experience. New York: Springer.

Polkinghorne, D. E. (2005). Language and meaning: Data collection in qualitative research. Journal of Counseling Psychology, 52, 137-145. http://dx.doi.org/10.1037/0022-0167.52.2.137

Polusny, M. A., Kehle, S. M., Nelson, N. W., Erbes, C. R., Arbisi, P. A., \& Thuras, P. (2011). Longitudinal effects of mild traumatic brain injury and posttraumatic stress disorder comorbidity on postdeployment outcomes in national guard soldiers deployed to Iraq. Archives of General Psychiatry, 68(1), 79-89.

Progoff, I. (1992). At a journal workshop: Writing to access the power of the unconscious and evoke creative ability. New York, NY: Tarcher.

Prudential. (2012). Veterans' employment challenges: Perceptions and experiences of transitioning from military to civilian life. Prudential.

QSR International (2012). NVivo 10. Available from:

http://www.qsrinternational.com/products_nvivo.aspx

[Accessed 30 August 2014]. 
Quillen-Armstrong, S. (2007). Course to help transition veterans into civilian life [Electronic Version]. Community College Times.

Radomski, M. V., \& Brininger, T. L. (2014). From the Desk of the Guest EditorsOccupational therapy for servicemember and veteran recovery, resilience, and reintegration: Opportunities for societal contribution and professional transformation. American Journal of Occupational Therapy, 68, 379-380. http://dx.doi.org/10.5014/ajot.2014.013060

Radomski, M. V., Davidson, L., Voydetich, D., \& Erickson. M. W. (2009). Occupational therapy for service members with mild traumatic brain injury. American Journal of Occupational Therapy, 64, 646-655.

Rausch, S. M., Gramling, S. E., \& Auerbach, S. M. (2006). Effects of a single session of large group meditation and progressive muscle relaxation training on stress reduction, reactivity, and recovery. International Journal of Stress Management, 13(3), 273-290. http://dx.doi.org/10.1037/1072-5245.13.3.273

Resnik, L., Gray, M., \& Borgia, M. (2011). Measurement of community reintegration in sample of severely wounded servicemembers. Journal of Rehabilitation Research \& Development, 48(2), 89-101. http://dx.doi.org/10.1682/JRRD.2010.04.0070

Resnik, L., Tian, F., Ni, P., \& Jette, A. (2012). Computer-adaptive test to measure community reintegration of Veterans. Journal of Rehabilitation Research \& Development, 49(4), 557-566. http://dx.doi.org/10.1682/JRRD.2011.04.0081

Rice, V. J., \& Gerardi, S. M. (1999). Part II. Work hardening for warriors: Training military occupational therapy professionals in the management of combat stress 
casualties. Work: A Journal of Prevention, Assessment and Rehabilitation, 13(3), 197-209.

Roebuck-Spencer, T. M., Vincent, A. S., Twillie, D. A., Logan, B. W., Lopez, C. M., Friedl, C.K., .....Gilliland, K. (2012). Cognitive change associated with selfreported mild traumatic brain injury sustained during the OEF/OIF conflicts. Clinical Neuropsychologist 26(3), 473-489.

Rogers, C. M., Mallinson, T., \& Peppers, D. (2014). High-Intensity sports for posttraumatic stress disorder and depression: Feasibility study of ocean therapy with veterans of Operation Enduring Freedom and Operation Iraqi Freedom. American Journal of Occupational Therapy, 68, 395-404. http://dx.doi.org/10.5014/ajot.2014.011221

Rudd, M. D., Goulding, J., \& Bryan, C. J. (2011). Student veterans: A national survey exploring psychological symptoms and suicide risk. Professional Psychology: Research and Practice, 42(5), 354-360. http://dx.doi.org/10.1037/a0025164

Rumann, C.B., Hamrick, F.A. (2009). Supporting Student Veterans in Transition. Wiley InterScience, 126, 25-34. http://dx.doi.org/10.1002/ss.313

Ryan, S. W., Carlstrom, A. H., Hughey, K. F., \& Harris, B. S. (2011). From boots to books: Applying Schlossberg's model to transitioning American veterans. NACADA Journal, 31(1), 55-63.

Sampson, J. P., Peterson, G. W., Lenz, J. G., Reardon, R. C., \& Saunders, D. E. (1996). Career thoughts inventory: Professional manual. Lutz, FL: PAR.

Sampson, J. P., Peterson, G. W., Lenz, J. G., Reardon, R. C., \& Saunders, D. E. (1998). The design and use of a measure of dysfunctional career thoughts among adults, 
college students, and high school students: The Career Thoughts

Inventory. Journal of Career Assessment, 6(2), 115-134.

http://dx.doi.org/10.1177/106907279800600201

Samuels, M. (1995). Art as a healing force. Alternative Therapies in Health and Medicine, 1(4), 38.

Santiago-Irizarry, V. (1996). Culture as cure. Cultural Anthropology, 11(1), 3-24.

Sayers, S. L., Farrow, V. A., Ross, J., \& Oslin, D. W. (2009). Family problems among recently returned military veterans referred for a mental health evaluation. Journal of Clinical Psychiatry, 70(2), 163.

Sayer, N. A., Friedemann-Sanchez, G., Spoont, M., Murdoch, M., Parker, L. E., Chiros, C., \& Rosenheck, R. (2009). A qualitative study of determinants of PTSD treatment initiation in veterans. Psychiatry, 72(3), 238-255.

Sayer, N. A., Noorbaloochi, S., Frazier, P., Carlson, K., Gravely, A., \& Murdoch, M. (2010). Reintegration problems and treatment interests among Iraq and Afghanistan combat veterans receiving VA medical care. Psychiatric Services, 61(6), 589-597.

Schlossberg, N. K. (1981). A model for analyzing human adaption to transition. Counseling Psychologist, 9(2), 2-18. http://dx.doi.org/10.1177/001100008100900202

Schlossberg, N. K., Lynch, A. Q., \& Chickering, A. W. (1989). Improving higher education environments for adults. San Francisco, CA: Jossey-Bass.

Schlossberg, N. K., Waters, E. B., \& Goodman, J. (1995). Counseling adults in transition (2nd ed.). New York, NY: Springer. 
Schwarzer, C., Starke, D., \& Buchwald, P. (2003). Towards a theory-based assessment of coping: The German adaption of the strategic approach to coping scale. Anxiety, Stress \& Coping, 16(3), 271-280.

Seal, K. H., Abadjian, L., McCamish, N., Shi, Y., Tarasovsky, G., \& Weingardt, K. (2012). A randomized controlled trial of telephone motivational interviewing to enhance mental health treatment engagement in Iraq and Afghanistan veterans. General Hospital Psychiatry, 34(5), 450-459. http://dx.doi.org/10.1016/j.genhosppsych.2012.04.007

Simmons, C. D., Griswold, L. A., \& Berg, B. (2010). Evaluation of social interaction during occupational engagement. American Journal of Occupational Therapy, 64, 10-17. http://dx.doi.org/10.5014/ajot.64.1.10

Skopp, N. A., Bush, N. E., Vogel, D. L., Wade, N. G., Sirotin, A. P., \& McCann, R. A. (2012). Development and initial testing of a measure of public and self-stigma in the military. Journal of Clinical Psychology, 68, 1036-1047.

Smith, T. C., Ryan, M. A., Wingard, D. L., Slymen, D. J., Sallis, J. F., \& KritzSilverstein, D. (2008). New onset and persistent symptoms of post-traumatic stress disorder self-reported after deployment and combat exposures: prospective population based US military cohort study. British Medical Journal, 336(7640), 366-371. http://dx.doi.org/10.1136/bmj.39430.638241.ae

Smith, D. L. (2014). The relationship between employment and veteran status, disability and gender from 2004-2011 Behavioral Risk Factor Surveillance System (BRFSS). Work-A Journal of Prevention Assessment \& Rehabilitation, 49(2), 325-334. http://dx.doi.org/10.3233/wor-131648 
Smith-Forbes, E., Najera, C., \& Hawkins, D. (2014). Combat operational stress control in Iraq and Afghanistan: Army occupational therapy. Military Medicine, 179(3), 279-284. http://dx.doi.org/10.7205/MILMED-D-13-00452

Smith-Osborne, A. (2012b). Supporting resilience in the academic setting for student soldiers and veterans as an aspect of community reintegration: The design of the Student Veteran Project study. Advances in Social Work, 13, 34-50.

Snead, K. M. \& Baridon, A. (2010). Community college support and engagement of servicemembers, veterans, and military families. White House Summit on Community Colleges. Available at: http://www2.ed.gov/PDFDocs/collegecompletion/12-community-college-support-and-engagement.pdf

Snyder, C. R., \& Pulvers, K. M. (2001). Dr. Seuss, the coping machine, and "oh the places you'll go”. In L. C. R. Snyder (Ed.), Coping with stress: Effective people and processes (pp. 3-29). New York, NY: Oxford University Press.

Søndergaard, M., \& Fisher, A. G. (2012). Sensitivity of the Evaluation of Social Interaction measures among people with and without neurologic or psychiatric disorders. American Journal of Occupational Therapy, 66, 356-362. http://dx.doi.org/10.5014/ajot.2012.003582

Speicher, S. M., Walter, K. H., \& Chard, K. M. (2014). Interdisciplinary residential treatment of posttraumatic stress disorder and traumatic brain injury: Effects on symptom severity and occupational performance and satisfaction. American Journal of Occupational Therapy, 68, 412-421. http://dx.doi.org/10.5014/ajot.2014.011304 
Spelman, J. F., Hunt, S. C., Seal, K. H., \& Burgo-Black, A. L. (2012). Post deployment care for returning combat veterans. Journal of General Internal Medicine, 27(9), 1200-1209.

Stachyra, A. (2011). Being and becoming a US Iraq war veteran: an exploration of the social construction of an emerging identity.

Stiglitz, J., \& Blimes, L. (2008). The three trillion dollar war. New York, NY: W.W. Norton and Co.

Stoller, C. C., Greuel, J. H., Cimini, L. S., Fowler, M. S., \& Koomar, J. A. (2012). Effects of sensory-enhanced yoga on symptoms of combat stress in deployed military personnel. American Journal of Occupational Therapy, 66, 59-68. http://dx.doi.org/10.5014/ajot.2012.001230

Strauss, A. L., \& Corbin, J. M. (1990). Basics of qualitative research. Newbury Park, CA: Sage.

Strauss, A., \& Corbin, J. (1998). Basics of qualitative research: Procedures and techniques for developing grounded theory. CA: Sage.

Substance Abuse and Mental Health Services Administration. (2012). SAMHSA's working definition of recovery: 10 guiding principles of recovery. Retrieved from http://content.samhsa.gov/ext/item?uri=/samhsa/content/item/10007447/1000744 7.pdf

Substance Abuse and Mental Health Services Administration. (2013). Results from the 2012 National Survey on Drug Use and Health: Mental Health Findings. NSDUH Series H-47, HHS Publication No. (SMA) 13-4805. Rockville, MD. 
Suls, J., \& Fletcher, B. (1985). The relative efficacy of avoidant and nonavoidant coping strategies: A meta-analysis. Health Psychology, 4(3), 249-288. http://dx.doi.org/10.1037//0278-6133.4.3.249

Tanielian, T. L., \& Jaycox, L. H. (Eds. 2008). Invisible wounds of war: Psychological and cognitive injuries, their consequences, and services to assist recovery. Santa Monica, CA: Rand Corporation. Available online at http://www.rand.org/pubs/monographs/2008/RAND_MG720.pdf

Tedeschi, R.G. (2011). Posttraumatic growth in combat veterans. J Clin Psychol Med Settings, 18, 137-144. http://dx.doi.org/10.1007/s10880-011-9255-2

Teclaw, R., Price, M. C., \& Osatuke, K. (2012). Demographic question placement: Effect on item response rates and means of a veterans health administration survey. Journal of Business and Psychology, 27(3), 281-290. http://dx.doi.org/10.1007/s10869-011-9249-y

Thompson, L. F., \& Surface, E. A. (2009). Promoting favorable attitudes toward personnel surveys: The role of follow-up. Military Psychology, 21(2), 139-161. http://dx.doi.org/10.1080/08995600902768693

Tolonon, H., Helakorpi, S., Talala, K., Helasoja, V., Martelin, T., \& Prättälä, R. (2006). 25 year trends and socio-demographic differences in response rates: Finnish adult health behavior survey. European Journal of Public Health, 21, 409-415. http://dx.doi.org/10.1007/s10654-006-9019-8

Tomar, N., \& Stoffel, V. (2014). Examining the lived experience and factors influencing education of two student veterans using photovoice methodology. American 
Journal of Occupational Therapy, 68(4), 430-438.

http://dx.doi.org/10.5014/ajot.2014.011163

United States Department of Veterans Affairs (2014). Education Program Beneficiaries.

Retrieved from

http://www.va.gov/VETDATA/docs/QuickFacts/Education_Beneficiaries.pdf

United States Department of Veterans Affairs (2009b). Post-9/11 GI Bill: Title 38 U.S.C.

Chapter 33. Washington, DC: Author.

U.S. Army Research Institute for the Behavioral and Social Sciences (ARI). (2005).

Reasons for leaving the AC Army before retirement. Fall 2004 sample survey of military personnel (ARI Survey Report No. 2005-03). Arlington, VA: Author.

U.S. Census Bureau. (2010). American Community Survey: Data on veterans. Retrieved from http://www.census.gov/hhes/veterans/data/

U.S. Department of Defense. (2015). Operation Iraqi Freedom and Operation Enduring Freedom casualty status. Retrieved from www.defense.gov/news/casualty.pdf

Vacchi, D. T. (2012). Considering student veterans on the twenty-first-century college campus. About Campus, 17, 15-21. http://dx.doi.org/10.1002/abc.21075

Vance, M. L., \& Miller, W. K. (2009). Serving Wounded Warriors: Current practices in postsecondary education. Journal of Postsecondary Education and Disability, 22(1), 18-28.

Vasterling, J.J., Brailey, K., Proctor, S.P., Kane, R., Heeren, T., \& Franz, M. (2012). Neuropsychological outcomes of mild traumatic brain injury, post-traumatic stress disorder and depression in Iraq deployed US Army soldiers. British Journal of Psychiatry, 201(3), 186-192. 
Veteran Benefits Act, 38 U.S.C. § 101 (1945). Retrieved from https://www.law.cornell.edu/uscode/pdf/uscode38/lii_usc_TI_38_PA_I_CH_1_S E_101.pdf

Viruell-Fuentes, E. A. (2007). Beyond acculturation: immigration, discrimination, and health research among Mexicans in the United States. Social Science \& Medicine, 65(7), 1524-1535.

Wada, M. (2011). Strengthening the Kawa model: Japanese perspectives on person, occupation, and environment. Canadian Journal of Occupational Therapy, 78(4), 230-236. http://dx.doi.org/10.2182=cjot.2011.78.4.4

Wang, C., \& Burris, M. A. (1997). Photovoice: Concept, methodology, and use for participatory needs assessment. Health Education \& Behavior, 24(3), 369-387.

Wertz, F. J. (2005). Phenomenological research methods for counseling psychology. Journal of Counseling Psychology, 52, 167-177. http://dx.doi.org/10.1037/00220167.52.2.167

Westwood, M. J., Black, T. G., \& McLean, H. B. (2002). A re-entry program for peacekeeping soldiers: Promoting personal and career transition.Canadian Journal of Counselling, 36(3), 221.

Wood, D. (2013, October 25). Veterans' college drop-out rate soars. The Huffington Post. Retrieved from http://www.huffingtonpost.com

Young, R. A., \& Collin, A. (2004). Introduction: Constructivism and social constructionism in the career field. Journal of vocational behavior, 64(3), 373388. http://dx.doi.org/10.1016/j.jvb.2003.12.005

Zambrana, R. E., \& Carter-Pokras, O. (2010). Role of acculturation research in 
advancing science and practice in reducing health care disparities among Latinos. American Journal of Public Health, 100(1), 18-23.

Zinger, L., \& Cohen, A. (2010). Veterans returning from war into the classroom: How can colleges be better prepared to meet their needs. Contemporary Issues in Education Research (CIER), 3(1), 39-52. 


\section{Vita}

Major Brian T. Gregg, MS, OTR/L, CHT

\section{General Information}

Place of Birth:

Fayetteville, N.C.

Certificate or Specialty Board Licensure:

Graduate Certificate in Clinical Research Skills, University of Kentucky August 6, 2015

National Board for Certification in Occupational Therapy: \#1072202

Board Certification in Hand Therapy: \#20120563

North Carolina State Licensure: \#5710

American Occupational Therapy Association: \#436530

American Society of Hand Therapists: \#38177

IRB Certification, CITI: \#3562676

\section{Education}

2013-Present The University of Kentucky, Lexington, KY Candidate, Doctor of Philosophy, Rehabilitation Sciences Anticipated graduation May 8, 2016

Dissertation: "An Exploration of Transition Experiences Shaping Student Veterans Life-Flow."

2006-2006 Troy University, Troy, AL

Master of Science in Sport and Fitness Management

2000-2004 Winston-Salem State University, Winston-Salem, NC Bachelor of Science in Occupational Therapy

\section{Professional Experience}

2011-2013 Chief occupational therapy services, William Beaumont Army Medical Center, Fort Bliss, TX

2010-2013 Clinical Instructor for occupational therapy services and Staff Therapist at William Beaumont Army Medical Center

2007-2010 Officer-In-Charge, COSC Fitness Team, 212 ${ }^{\text {th }}$ Combat and Operational Stress Control Detachment, Fort Campbell, KY 
2009-2010 (April-April) Officer-In-Charge, COSC Fitness Team,

Prevention Team, and on-site Principal Investigator, 212th Combat and Operational Stress Control Detachment, deployed overseas to Iraq

2008-2008 (July-October) Detachment Commander, 212 $2^{\text {th }}$ Combat and Operational Stress Control, Fort Campbell, KY

2008-2008 (March-July) Rear Detachment Commander, $86^{\text {th }}$ Combat Support Hospital, Fort Campbell, KY

2007-2007 (July-Sep) Chief occupational therapy services, Martin Army Community Hospital, Fort Benning, GA

2005-2007 (July-Sep) Staff Occupational Therapist, Martin Army

Community Hospital, Fort Benning, GA

\section{Teaching Activity}

Eastern Kentucky University - Teaching Apprenticeship, 2014

OTS 852 Occupational Therapy in Mental Health Settings

University of Kentucky - Guest Lecture, 2014

ATC 690 Athletic Training Orthopedic Evaluation of the Upper Extremity

\section{Professional Publications}

\section{Peer-Reviewed Manuscripts}

Gregg, B.T., Howell, D.M., Quick, C.D., \& Iwama, M.K. (2015). The Kawa River Model: Applying Theory to Develop Interventions for Combat and Operational Stress Control. Occupational Therapy in Mental Health, 31(4), 366384.

Gregg, B. T. (2011). Crossing the berm: An occupational therapist's perspective on animal-assisted therapy in a deployed environment. US Army Medical Department Journal, 55-56.

\section{Manuscripts in Peer-Review}

Gregg, B., Kitzman, P., \& Shordike, A. (In press). Well-being and coping of student veterans readjusting into academia: A pilot survey. Occupational Therapy in Mental Health.

Gregg, B., Howell, D., \& Shordike, A. (In press). Experiences of veterans transitioning into academia. American Journal of Occupational Therapy. 


\section{Invited Speaking Engagement/Presentations}

\section{Peer-Review-National}

Gregg, B.T., Shordike, A., Howell, D., \& Kitzman, P. (2015, April). Well-being and Coping of Military Veterans Readjusting into Academia, $2^{\text {nd }}$ Poster results presentation at the American Occupational Therapy Association Annual Conference, Nashville, TN

Gregg, B.T., Shordike, A., Howell, D., Kitzman, P. (2015, April). The Experiences of Student Veterans Transitioning into Academia: A Phenomenological Study, Poster presentation at the American Occupational Therapy Association Annual Conference, Nashville, TN

Najera, C, Goldman, S, Gregg, B, Dougherty, D, Norris, D, Fike, L. (2011, April). Deployment Risk and Resilience Factors in U.S. Soldiers Referred to an Occupational Therapy Program to Address Combat Stress while Serving in Operation Iraqi Freedom. Poster presentation at AOTA 91st Annual Conference \& Expo, Philadelphia, PA

\section{Peer-Review-Local}

Gregg, B.T., Shordike, A., Howell, D., Kitzman, P. (2015, November). The Experiences of Student Veterans Transitioning into Academia: A Phenomenological Study, Platform presentation at Eastern Kentucky University Research Day, Richmond, KY

Smith-Forbes, E.V., Gregg, B.T., Reer, T. (2014, September). The Deployment Role of Army Occupational Therapy, Panel presentation at the Kentucky Occupational Therapy Association Annual Conference, Lexington, KY

Gregg, B.T., Shordike, A., Howell, D., Kitzman, P. (2015, March). Well-being and Coping of Military Veterans Readjusting into Academia, $1^{\text {st }}$ Poster results presentation at the $10^{\text {th }}$ Annual Center for Clinical Translational Sciences Spring Conference, Lexington, KY

Gregg, B.T., Shordike, A., Howell, D., Kitzman, P. (2015, April). The Experiences of Student Veterans Transitioning into Academia: A Phenomenological Study, $2^{\text {nd }}$ Poster presentation at the University of Kentucky College of Health Sciences Colloquium, Lexington, KY

Gregg, B.T., Shordike, A., Howell, D., Kitzman, P. (2014, November). Wellbeing and Coping of Military Veterans Readjusting into Academia, $2^{\text {nd }}$ Poster abstract presentation at Eastern Kentucky University Research Day, Richmond, KY 
Gregg, B.T., Shordike, A., Howell, D., Kitzman, P. (2014, March). Well-being and Coping of Military Veterans Readjusting into Academia, ${ }^{\text {st }}$ Poster abstract presentation at the Kentucky Appalachian Rural Rehabilitation Network Annual Conference, Lexington, KY

\section{Local Invitations}

Gregg, BT. (2015, July). Lecture. Psychosocial considerations of combat Veterans involved in community reintegration. Doctoral of Physical Therapy program, Bellarmine University, Louisville, Kentucky.

Gregg, BT. (2013, October). Lecture. Neuromuscular assessment of the upper extremity. Doctoral of Physical Therapy program, University of Texas at El Paso, El Paso, Texas. 